\title{
The use of Information and Communication Technology in Teaching and Learning within Higher Education Sector of a Small Island Developing State: The Case of the Maldives
}

\author{
by
}

Mohamed Kinaanath

A thesis submitted in fulfilment of the requirements for the degree of

Doctor of Philosophy in Information Systems 


\section{ABSTRACT}

This study sought to a) discover the specific factors that influence Information and Communications Technology (ICT) use in higher education teaching and learning in Small Island Developing States (SIDS) such as the Maldives; b) determine how ICT can be used within higher education in SIDS; c) develop a framework / model for ICT adoption in higher education in teaching and learning of SIDS; and d) use UNESCO's continuum model of ICT development and the TOEG framework to ground these factors to produce a practical roadmap.

The Maldives was chosen as a representative case for SIDS in this research. The Maldives consists of 1,192 coral islands with 198 inhabited islands. Due to its small population densities spread over remote islands, distribution of higher education access has been a major impediment in the Maldives, calling for the use of ICT.

This research utilised an interpretive paradigm with qualitative research methods (interviews; focus group discussions; qualitative survey and document reviewing). The case research methodology provided a variety of perspectives; enabled multiple data collection techniques; and examined technology integration within a technology rich environment. The research participants were students, teachers, senior academic managers, education and information technology consultants / experts in the Maldives.

The findings revealed the specific factors that affect the ICT adoption within higher education teaching and learning in the context of the Maldives. An Extended Technology Acceptance Model (TAM) of ICT use in higher education in SIDS was developed using TAM theory, Diffusion of Innovation (DOI) theory and a TOEG (Technology-Organisational-Environment-Geography) framework.

The technology context examined government support for Information Technology, ICT support for teachers, ICT Infrastructure, underutilisation of ICT resources, perception of benefits from the present ICT infrastructure and ICT 
policies. The organisational context included finance, centralisation, human resources, top management support, ICT confidence among teachers, and research. The environmental context contained social issues (drugs and overcrowding) and cultural issues (gender disparity, cultural homogeneity among students and teachers and no culture of using ICT in academic learning, and a spoon-feeding pedagogical tradition). Finally the geography context identified issues associated with geography, transportation and the digital divide between remote islands.

These factors impede the adoption of ICT in the higher education sector in SIDS such as the Maldives. A practical roadmap was formulated by utilising the United Nations Educational, Scientific and Cultural Organization (UNESCO) continuum model of ICT development and the TOEG framework to recommend four broad stages in terms of adoption and use of ICT in higher education.

This study contributes to existing knowledge by providing a clear understanding of the present role of ICT as well as information on how ICT can be used in higher education in SIDS such as the Maldives. This research is important to gain a wider understanding of the future directions for adoption of ICT within higher education in SIDS. The research will fill critical gaps in the current research, expand the coverage of relatively neglected research areas in SIDS, contribute to practitioners and the academic community, and stimulate further debate. 


\section{ACKNOWLEDGEMENTS}

My first thanks go to Almighty Allah and his Messenger Prophet Mohamed (Peace be upon Him), for giving me the courage and ability to complete my doctoral studies. Without the kind blessing of Almighty Allah this long journey would have been impossible.

I am extremely grateful to my primary supervisor Associate Professor Dr. Hans Lehmann who has been helpful and supportive during my long journey. He has been inspirational and gave constructive and valuable comments. My sincere thanks go to my secondary supervisor Dr. Brian Harmer, whose insightful timely comments gave me the opportunity to finish my thesis.

Before, the completion of my PhD thesis Dr. Brian Harmer retired from his job. After his retirement, a new secondary supervisor, Mr. Alastair Smith, who is working as a Senior Lecture at School of Information Management, was assigned. I would also like to thank Mr. Alastair Smith for his priceless comments on my research and useful guidance provided during the completion stages of my thesis.

My special thanks goes to the chairman of the Villa Foundation Honourable Mr. Qasim Ibrahim, for his kind financial assistance for my PhD studies. Without his help, my long journey would not have been possible.

I would also like to thank the heads of the three higher education institutes who helped me in the data collection process: the Rector of Villa College, Dr. Ahmed Anwar; the Rector of the Maldives College of Higher Education, Dr. Hassan Hameed; and the President of Clique College, Mr. Naushad Mohamed. I also thank freelance education consultant Dr. Ahmed Shareef for his kind assistance in providing useful information during the course of my PhD programme.

I am also grateful to the research participants who actively participated in the sessions. I would like to acknowledge the interviewees, focus group participants and qualitative survey participants who helped me in getting the required data for 
my research. I also thank the skilled moderator, Mr. Muhammad Azim Abdul Haadi who worked in undertaking the four focus group sessions.

I would also like to thank the academic and administrative staff members of the School of Information Management at Victoria University of Wellington. The administrative staff were very helpful in ironing out administrative arrangements and making my studies more comfortable.

I would like to thank my beloved parents for their continued support during my studies. My father (Mr. Abdul Hameed Abdul Wahhab) and my mother (Ms. Aminath Hanim) were always available during times of great difficulties. In addition, I thank my mother-in-law and father-in-law for their continued support during the entire journey.

Finally, my sincere and heartfelt thanks go to my wife, Ms. Ruzna Mohamed. She was the most inspirational figure throughout my PhD studies. She has been helpful and patient when I was working hard during the evenings. She also has worked extremely hard to provide me with the needed financial backing at times of hardship during my studies.

With all these I would like to dedicate my PhD thesis to my parents and wife. 


\section{ACRONYMS}

ADB

ADSL

CAM

CD

CD-ROM

CCS

DHET

DNP

DOI

DVD

EDC

EMIS

GDP

GEMS

GER

GNM

HEC

HEI

HRCM

ICT

IDC

ISP

ISDN

IS

IT

ITU

KBPS

LDC

LMS

MCHE

MCST

MCT

MCTC

MCU

MDG

MMS

MNQF

MNU

MOOC

MQA
Asian Development Bank

Asymmetric Digital Subscriber Line

Communication Authority of Maldives

Compact Disk

Compact Disk Read Only Memory

Citizen Centric Solutions

Department of Higher Education and Training

Department of National Planning

Diffusion of Innovation

Digital Versatile Disk

Education Development Centre

Education Management Information System

Gross Domestic Product

Government E-letter Management System

Gross Enrollment Rate

Government Network of Maldives

Human Ethics Committee

Higher Education Institutions

Human Rights Commission of the Maldives

Information and Communications Technology

International Data Corporation

Internet Service Provider

Integrated System Digital Network

Information Systems

Information Technology

International Telecommunication Union

Kilo Bits Per Second

Least Developed Countries

Learning Management Systems

Maldives College of Higher Education

Ministry of Communication, Science and Technology

Mobile Communications Technology

Multi-Purpose Community Tele-Centre

Multipoint Control Unit

Millennium Development Goals

Multimedia Messaging Service

Maldives National Qualification Framework

Maldives National University

Massive Open Online Course

Maldives Qualifications Authority 
MTCA

NCIT

NDP

PEOU

PU

PITA

ROI

RSS

SAARC

SIDS

SIM

SMS

TAM

TAM

TOE

TOEG

TV

TVM

TVET

UN

UNCTAD

UNDP

UNESCO

UNESCAP

UNICEF

USA

UK

VIHE

VOIP

WWW
Ministry of Tourism and Civil Aviation

National Centre for Information Technology

National Development Plan

Perceived Ease Of Use

Perceived Usefulness

Pacific Islands Telecommunication Association

Return On Investment

Real Simple Syndication

South Asian Association for Regional Cooperation

Small Islands Developing States

School of Information Management

Short Message Service

Technology Acceptance Model

Telecommunication Authority of Maldives

Technology-Organisation-Environment

Technology-Organisation-Environment-Geography

Television

Television Maldives

Technical Vocational Education Training

United Nations

United Nations Conference on Trade and Development

United Nations Development Programme

United Nations Educational, Scientific and Cultural Organization

United Nations Economic and Social Commission for Asia and the Pacific

United Nations International Children's Emergency Fund

United States of America

United Kingdom

Virtual Island Higher Education

Voice Over Internet Protocol

World Wide Web 


\section{LIST OF TABLES}

Table 1: $\quad$ Model of Distance Education (adapted from Taylor, 1994, p.2) ................30

Table 2: Some studies based on TOE framework............................................................. 38

Table 3: Atolls populations and distance to Male' .........................................................93

Table 4: Number of islands by size of population ..........................................................96

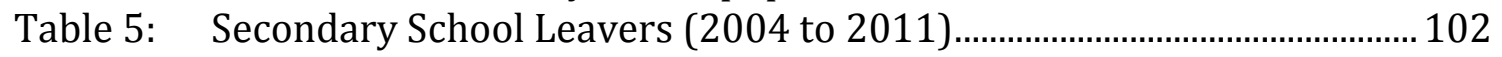

Table 6: Student numbers at seven higher education institutes..............................103

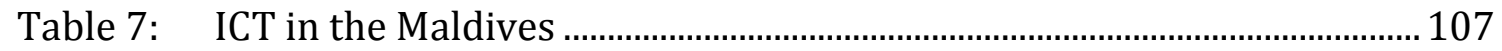

Table 8: The twenty most dynamic economies in terms of mobile penetration

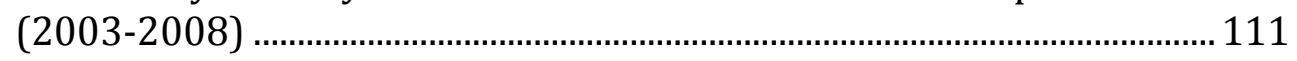

Table 9: $\quad$ ICT in South Asian Countries (SAARC countries) ....................................... 113

Table 10: SIDS geographical context with ICT............................................................ 115

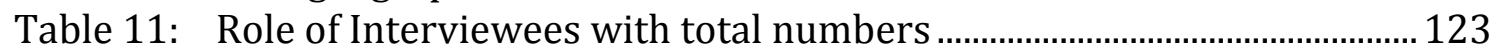

Table 12: Summary of focus group members ................................................................ 124

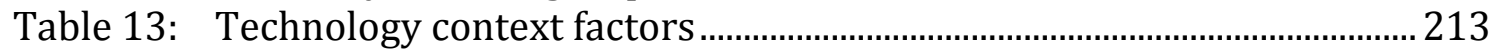

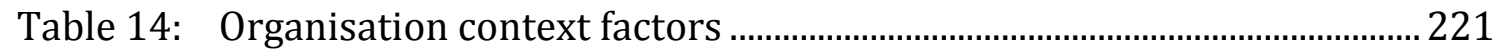

Table 15: Environment Context Factors .................................................................... 227

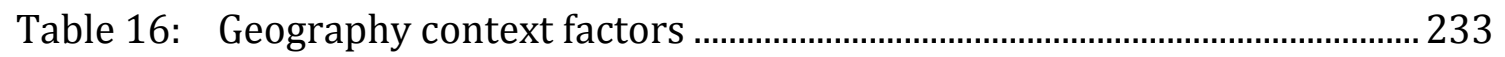

\section{LIST OF FIGURES}

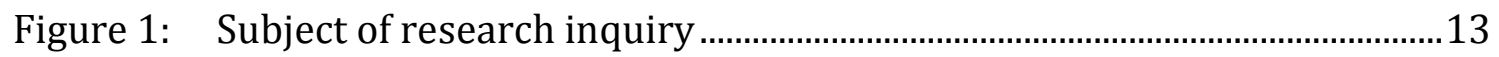

Figure 2: TOE framework (Source: Tornatzky \& Fleischer, 1990, p.154) ................37

Figure 3: $\quad$ Original Technology Acceptance Model (Davis et al. 1989, p.985) .........50

Figure 4: Rogers' Innovation adoption Curve (adapted from: Rogers, 2003, p.281)

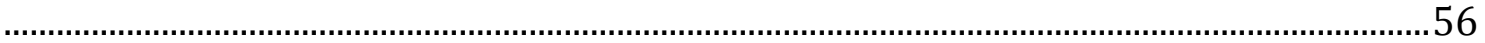

Figure 5: Diffusion of Innovation (Rogers, 2003, p.411) ...........................................58

Figure 6: UNESCO's Continuum for ICT development (UNESCO, 2002, p.14) ......60

Figure 7: Models of stages of ICT development (UNESCO, 2002, p.17) ....................61

Figure 8: Conceptual Model of this research..................................................................63

Figure 9: Data collection methods used in this research ..........................................75

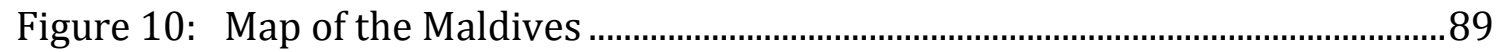

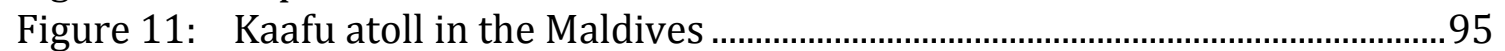

Figure 12: Higher Education System in the Maldives ...............................................104

Figure 13: Maldives National Qualification Framework (MNQF) ............................. 105

Figure 14: Internet users with 100 per inhabitants ................................................... 108

Figure 15: Mobile coverage in South Asian Countries (SAARC countries).............113

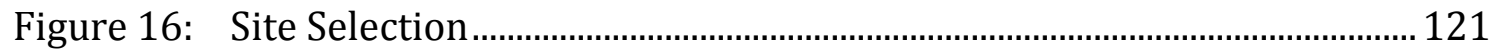

Figure 17: Data analysis process (adapted from Punch, 2005 and Neuman, 2011)

129

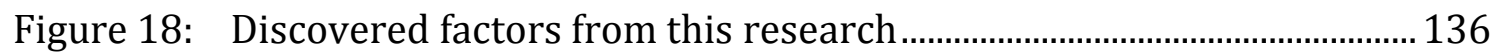

Figure 19: How often do you use Internet? .................................................................... 142

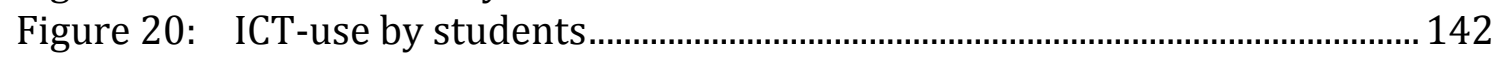

Figure 21: ICT use as a learning tool by students....................................................... 144

Figure 22: TOEG framework - adapted from original TOE framework (Tornatzky

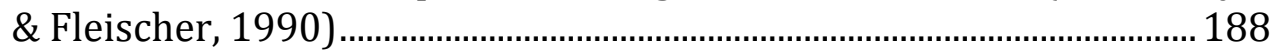

Figure 23: Traditional method of knowledge transfer............................................... 197 
Figure 24: Adapted TAM using TOEG framework and DOI theory

Figure 25: Practical roadmap by grounding UNESCO's continuum for ICT adoption with TOEG framework (adapted from: UNESCO, 2002)..... 237

Figure 26: Three-tiered approach for ICT use 245

\section{APPENDICES}

Appendix 1: Interview Questions and Guidelines..................................................... 291

Appendix 2: $\quad$ Focus Group Questions and Guidelines ................................................. 293

Appendix 3: Sample Qualitative Survey Form............................................................ 296

Appendix 4: Information sheet for Qualitative Survey with Consent .................... 298

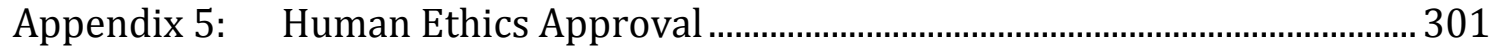

Appendix 6: $\quad$ Request for participation for Higher Education Institutes ............... 302

Appendix 7: Consent of Higher Education Institutes...................................................306

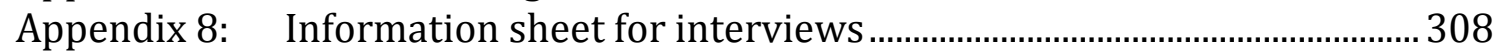

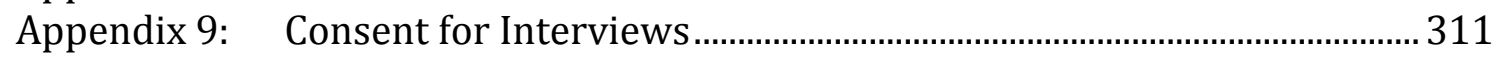

Appendix 10: Information sheet for Focus Group Discussion.....................................313

Appendix 11: Consent Focus Group Discussions............................................................. 316

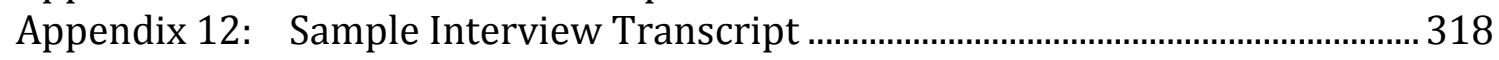

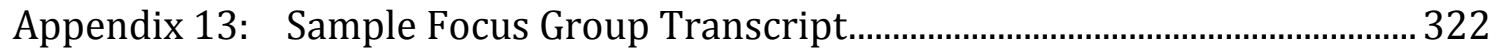

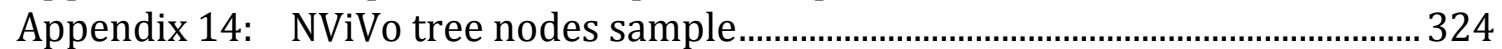

Appendix 15: Conceptualisation Sample Diagrams.......................................................... 325 


\section{TABLE OF CONTENTS}

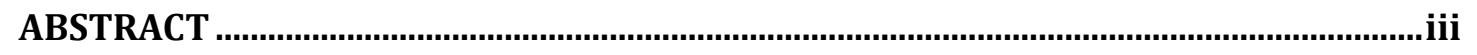

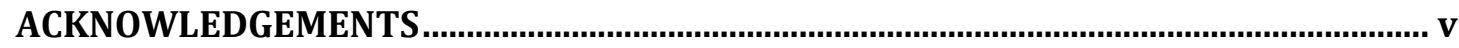

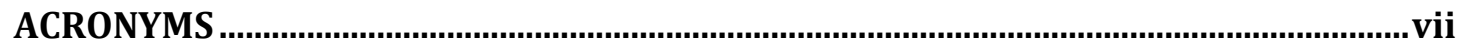

LIST OF TABLES

LIST OF FIGURES

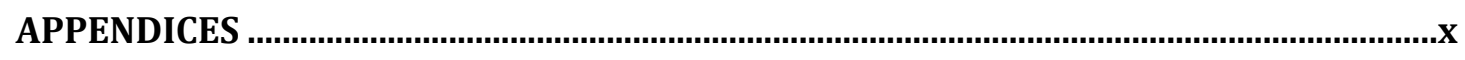

1 INTRODUCTION

$1.1 \quad$ Research Background..................................................................................... 1

1.2 Statement of the problem ............................................................................... 2

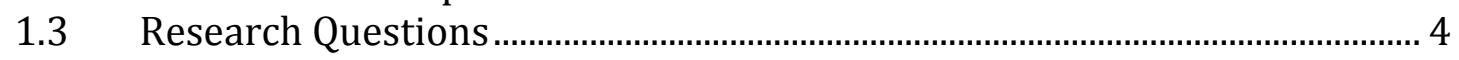

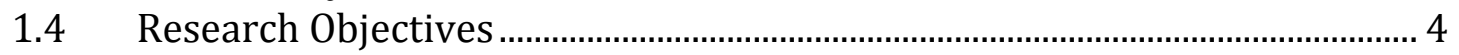

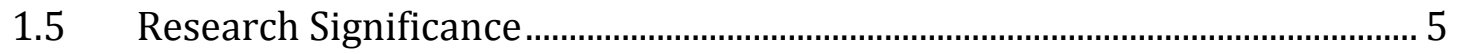

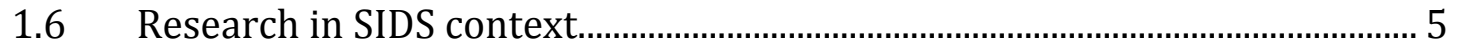

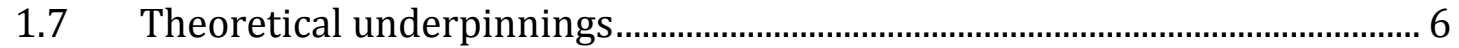

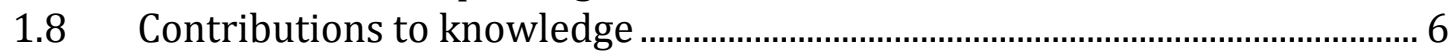

1.9 Structure of this thesis ........................................................................................ 7

1.10 Definition of key terms ................................................................................... 10

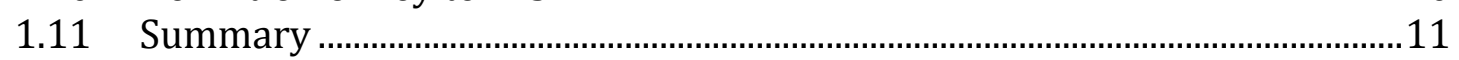

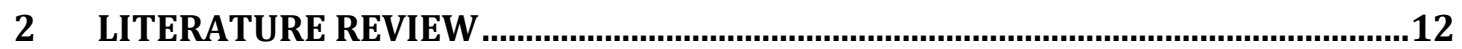

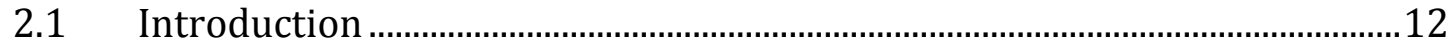

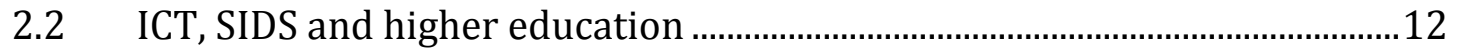

2.2.1 Small Island Developing States (SIDS) ........................................................

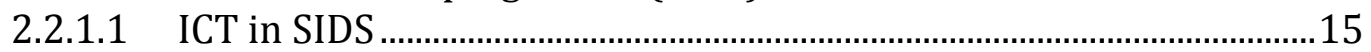

2.2.1.2 SIDS in higher education......................................................................16

2.2.2 Information and Communication Technologies (ICT) ...............................17

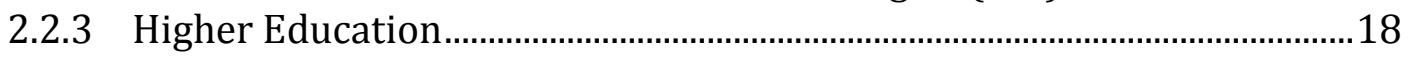

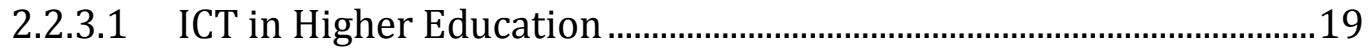

2.3 Information and Communications Technology as media................................22

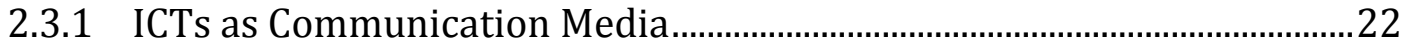

2.3.2 ICTs as Collaborative Media ..........................................................................23

2.3.3 ICTs as Constructive Media ...................................................................2 24

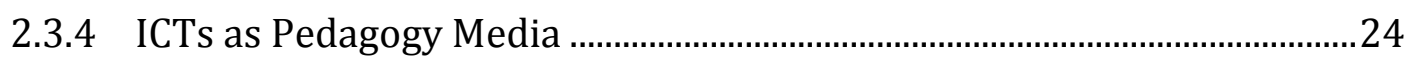

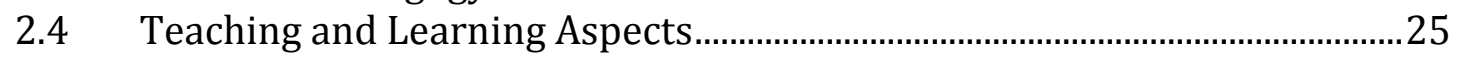

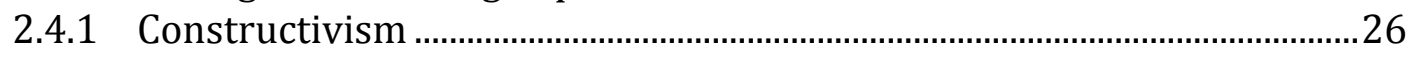

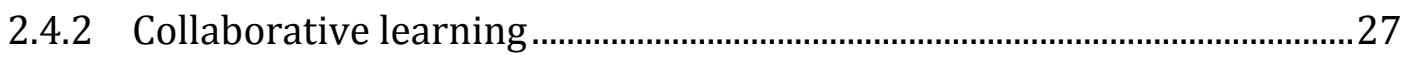

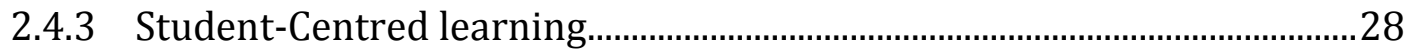

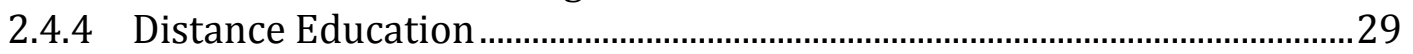

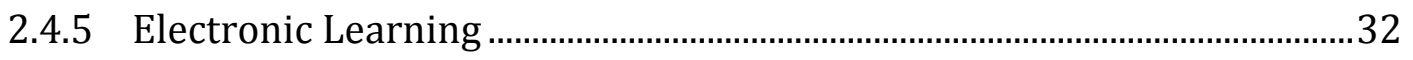

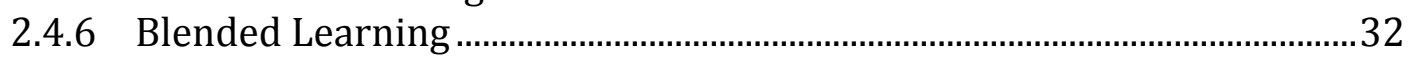

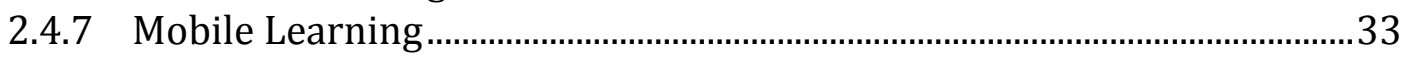




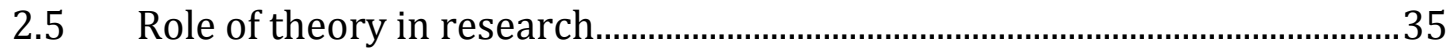

2.5.1 Theories and frameworks.........................................................................35

2.6 Technology-Organisation-Environment (TOE) framework ............................36

2.6.1 TOE framework elements .................................................................................

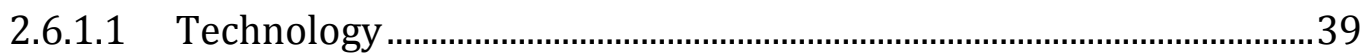

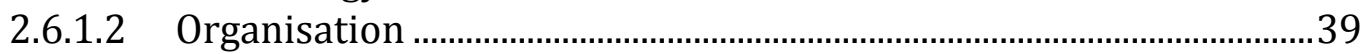

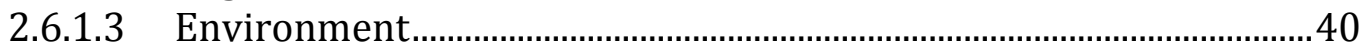

2.7 ICT-higher education in the Technology Context............................................40

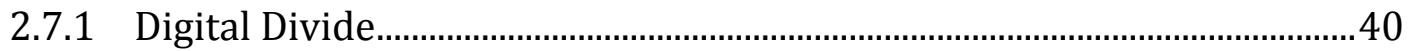

2.7.2 Infrastructure and resources.........................................................................4

$2.8 \quad$ ICT-higher education in the Organisation Context ...........................................42

2.8.1 Human Resources ............................................................................................42

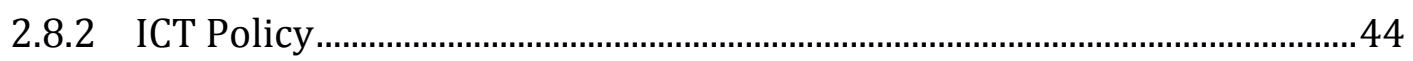

2.8.3 Organisational Support and Systemic issues...............................................4

2.8.4 Lack of confidence and competency of teachers........................................4

2.9 ICT-higher education in the Environment Context............................................46

2.9.1 Socio-culture ...................................................................................................46

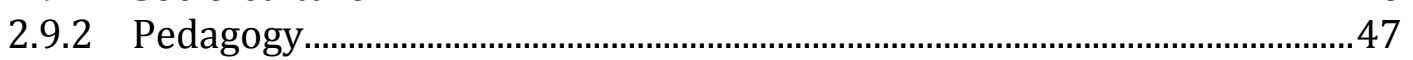

2.9.3 Attitude towards technology ……………………………………………......4

2.10 Technology Acceptance Model (TAM) ...............................................................49

2.10.1 Perceived Ease of Use (PEOU) ..................................................................51

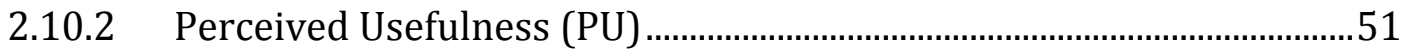

2.10.3 Attitude towards using technology …………………………………….....52

2.10.4 Behavioural intention of use .................................................................52

$2.10 .5 \quad$ Justification of TAM ……………………………………………………...52

2.11 Diffusion of Innovation (DOI) ...........................................................................53

2.11 .1 Innovation .............................................................................................53

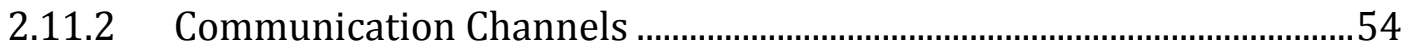

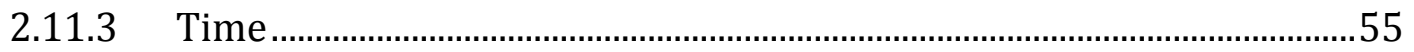

2.11.4 Adopter Categories ..................................................................................56

2.11.5 Social System.........................................................................................5

2.11.6 Justification of DOI to this study .............................................................57

2.11.7 Organisational Innovation ......................................................................58

2.11.8 Justification of Organisational Innovation................................................59

2.12 UNESCO's continuum model of ICT development...........................................59

2.12.1 Stages of teaching and learning through ICT …………………………...61

2.13 Conceptual Model of this research ...................................................................62

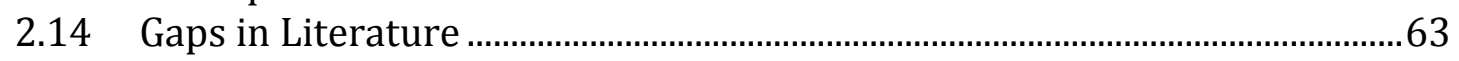

2.15 Chapter Summary …………………………………….........................................64

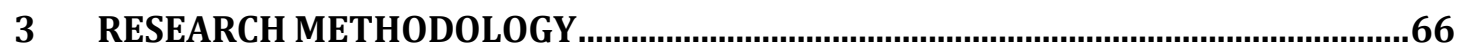

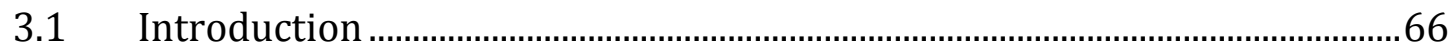

3.2 The choice of methodology …………………………………………………....66

3.3 Research Design Considerations......................................................................67

3.3.1 Interpretive Paradigm ...................................................................................67

3.3.2 Qualitative Approach ................................................................................69

3.3.3 Linking research paradigm and methodology to research questions ...69

3.3.4 The Research Methodology: Case Research ……………………………….....70

3.3.4.1 Exploratory Case Research ......................................................................72 


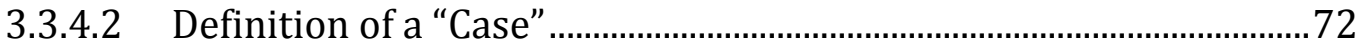

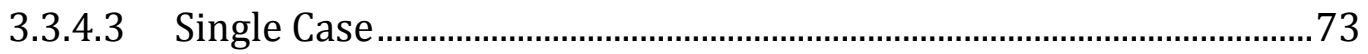

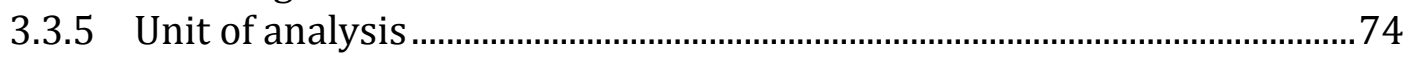

3.3.6 Nature of data required to answer the research questions.......................75

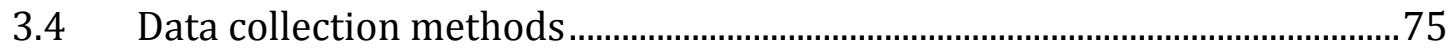

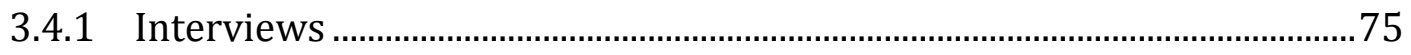

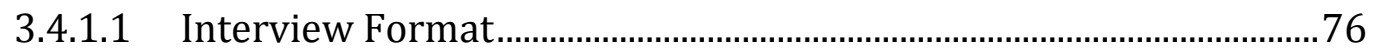

3.4.1.2 Selecting interview participants.........................................................

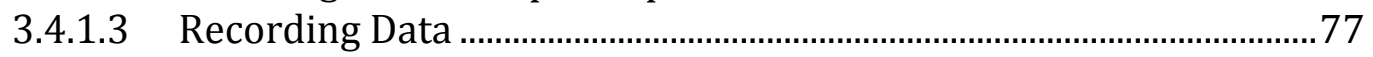

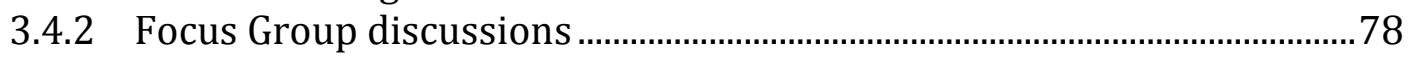

3.4.2.1 Structure of Focus Groups.................................................................... 78

3.4.2.2 Selecting focus group participants........................................................ 79

3.4.2.3 Undertaking a focus group discussion ................................................. 79

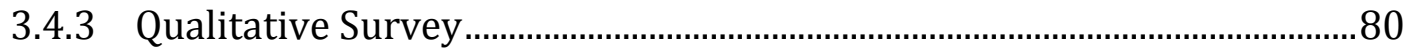

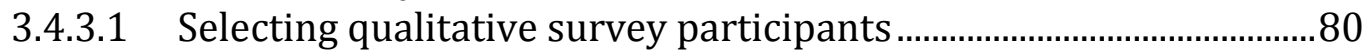

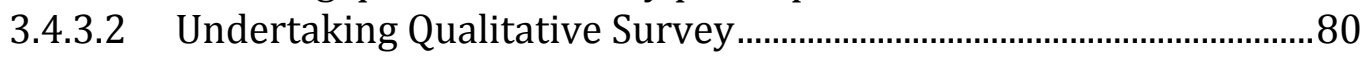

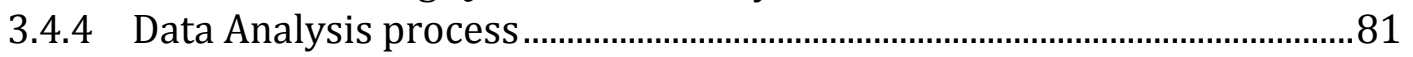

3.4.5 Document Reviewing (Secondary data) .......................................................... 81

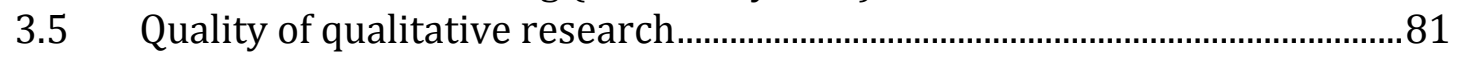

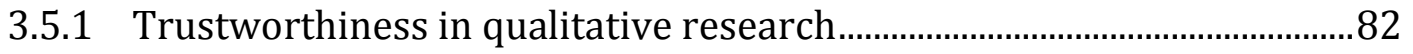

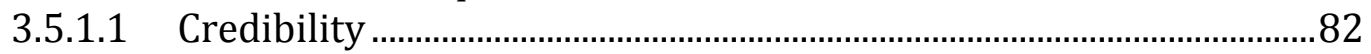

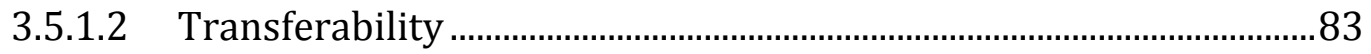

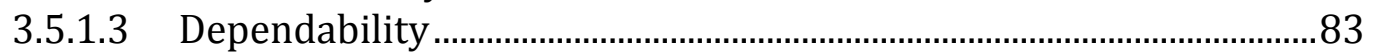

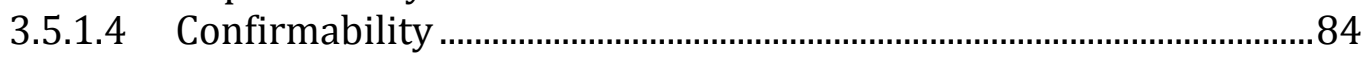

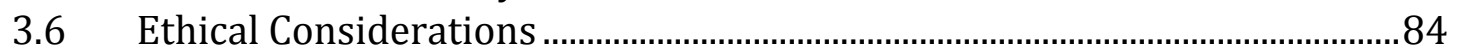

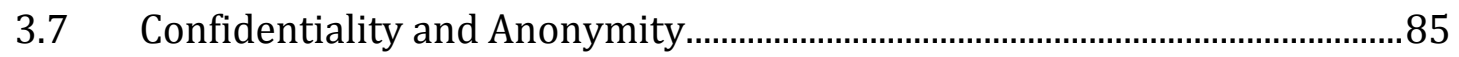

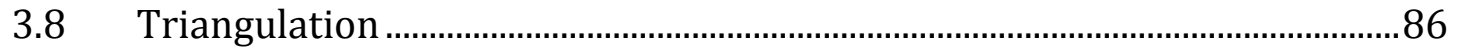

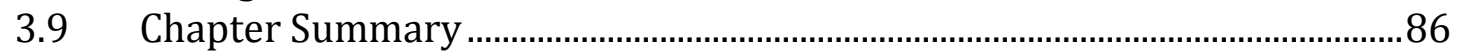

4 CASE OF THE MALDIVES

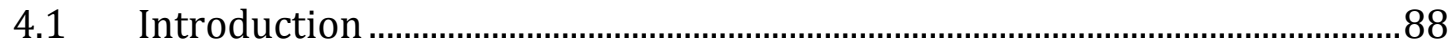

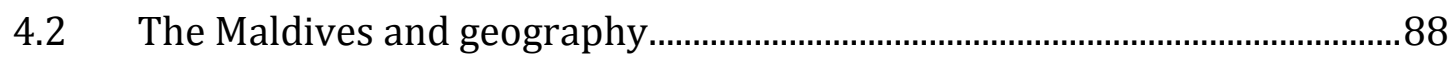

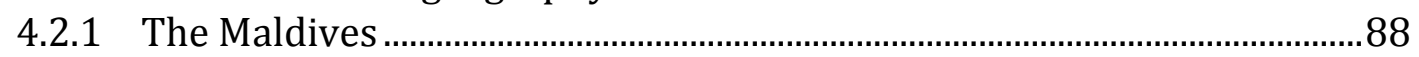

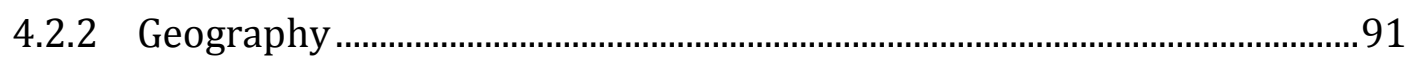

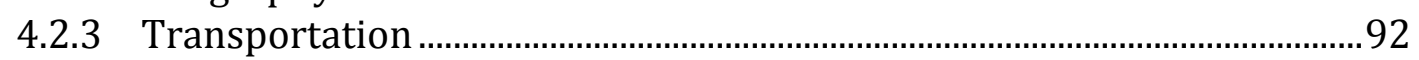

4.2.4 Capital - Male' and remote islands..................................................................94

4.3 Maldives' economy, population and governance.................................................95

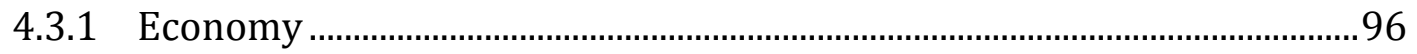

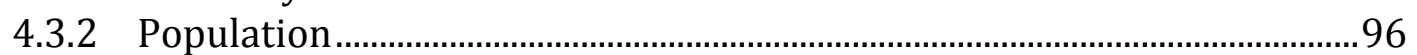

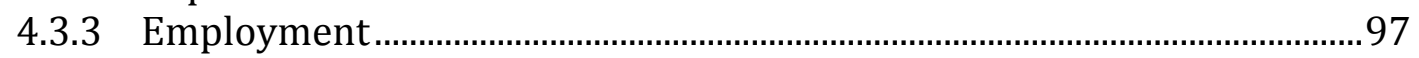

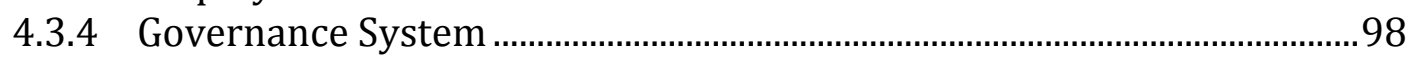

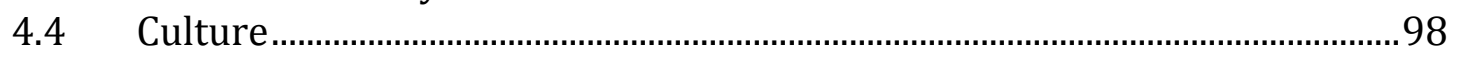

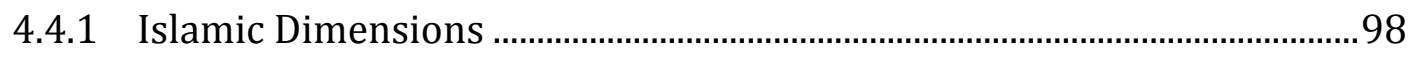

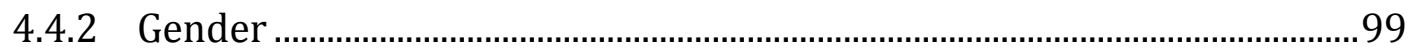

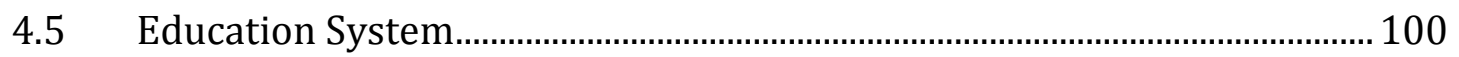

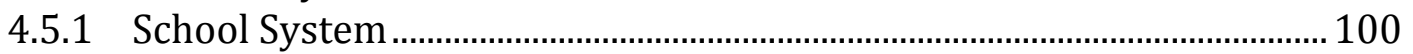

4.5.2 Higher Education in the Maldives................................................................ 101

4.5.3 Maldives National Qualification Framework (MNQF) ..............................104

4.6 Information and Communications Technology (ICT) ................................... 106 


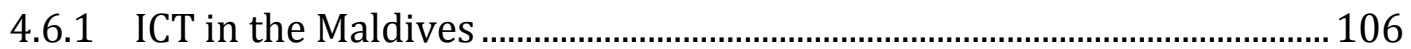

4.6.2 ICT Projects in the Maldives.......................................................................... 108

4.6.3 ICT infrastructures ................................................................................. 110

4.6.4 The Maldives compared with regional countries with regard to ICT.112

4.6.5 SIDS comparison in terms of geography and ICT ......................................114

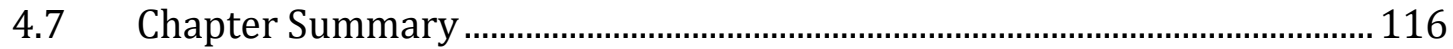

5 RESEARCH PROCEDURE

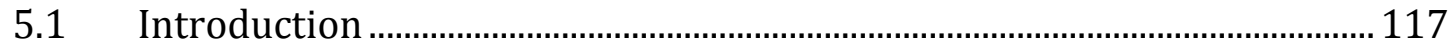

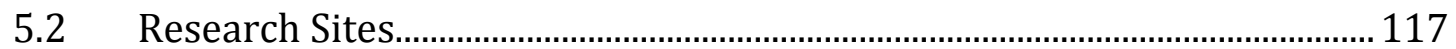

5.2.1 Short listed Data Collection at Higher Education Institutes ................... 117

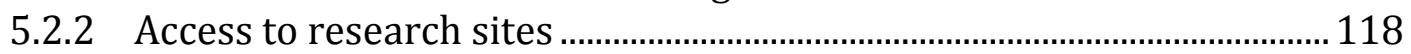

5.2.2.1 Maldives College of Higher Education .............................................. 119

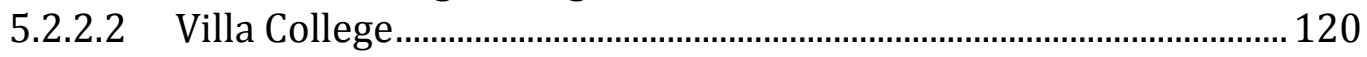

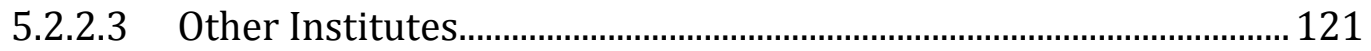

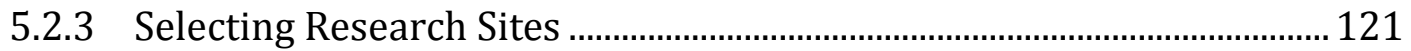

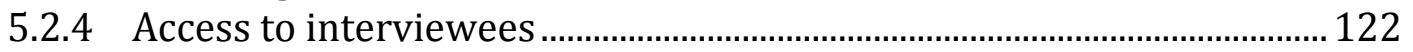

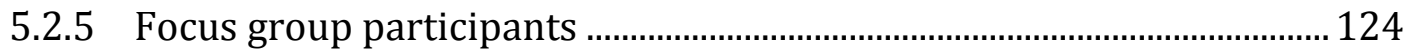

5.2.6 Qualitative Survey participants .................................................................. 126

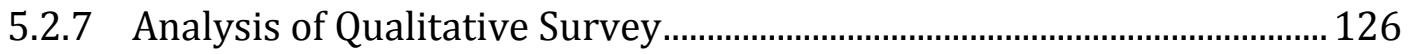

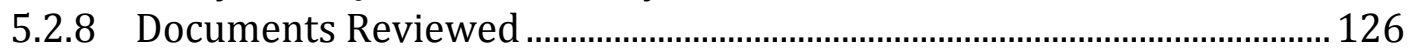

5.2.9 Informed Consent......................................................................................... 127

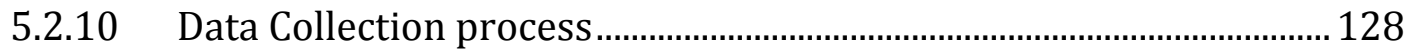

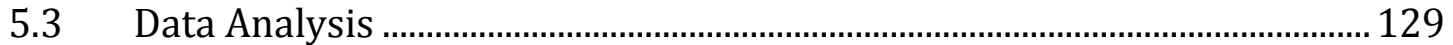

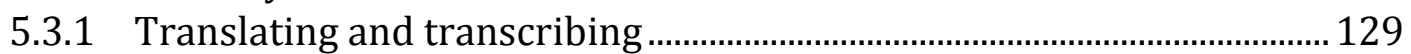

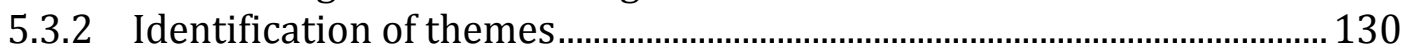

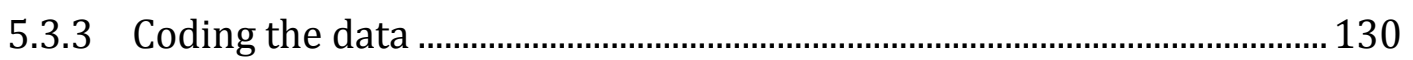

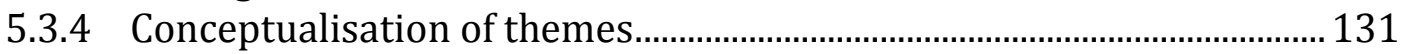

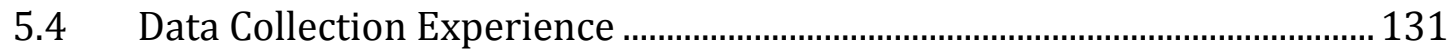

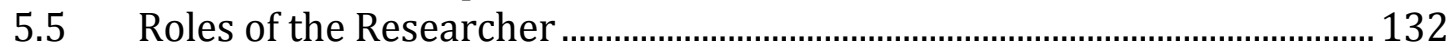

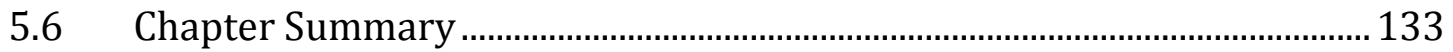

6 RESULTS - SPECIFIC FACTORS DISCOVERED

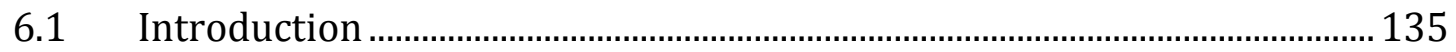

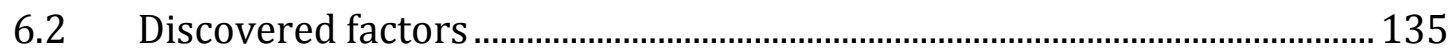

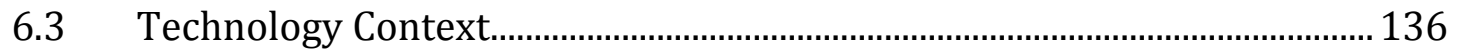

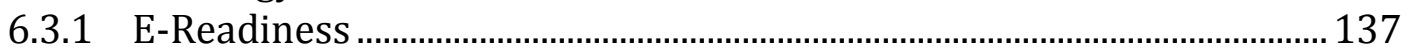

6.3.1.1 Government Support for Information Technology..........................137

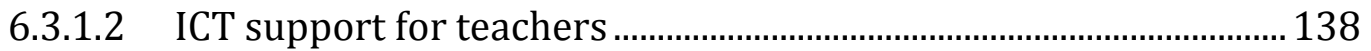

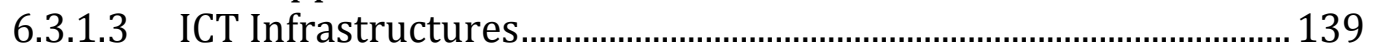

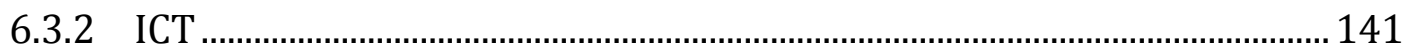

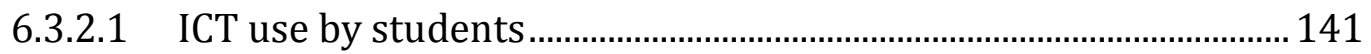

6.3.2.2 ICT resources underutilised ............................................................. 144

6.3.2.3 Perception of benefits from the present ICT infrastructure ......... 146

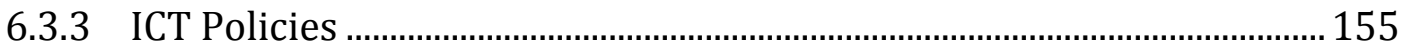

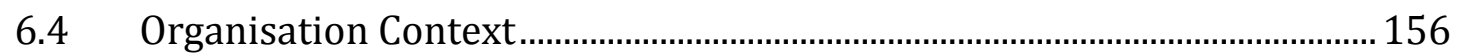

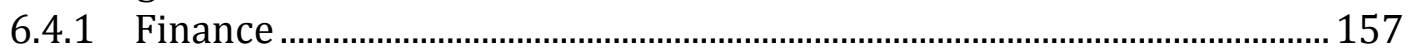

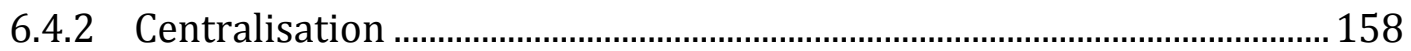

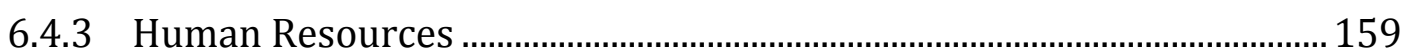




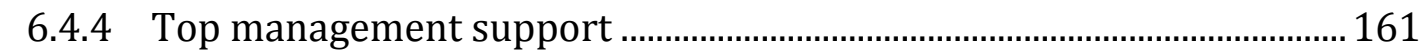

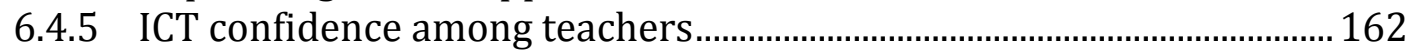

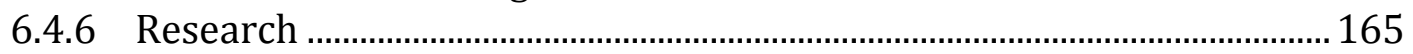

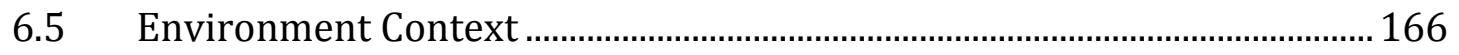

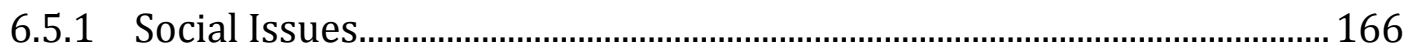

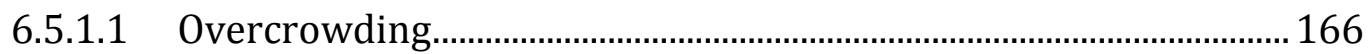

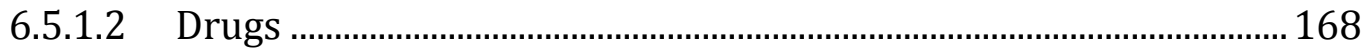

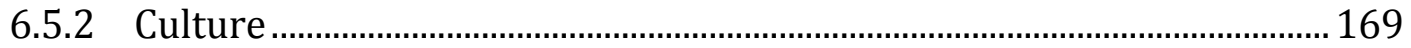

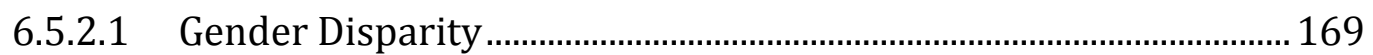

6.5.2.2 Cultural homogeneity among students and teachers ...................... 171

6.5.2.3 No culture of using ICT in academic learning ................................. 172

6.5.3 Pedagogy practices .................................................................................... 174

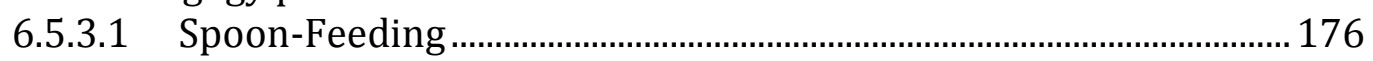

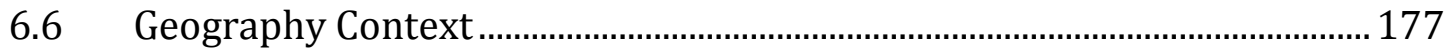

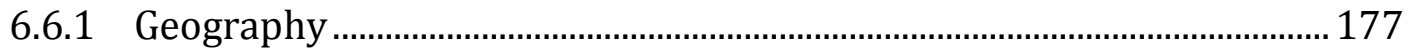

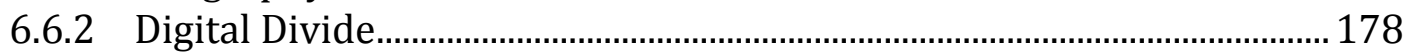

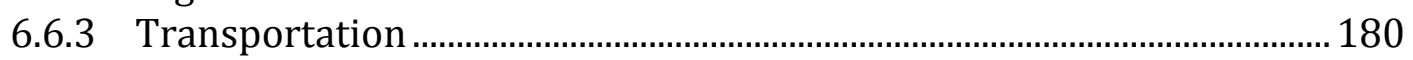

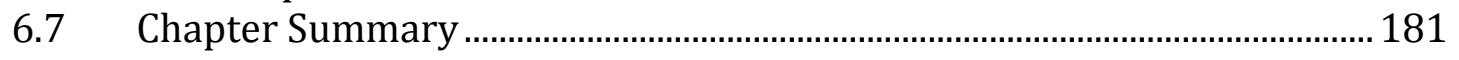

7 ANALYSIS - HOW CAN ICT BE USED IN HIGHER EDUCATION ............................. 183

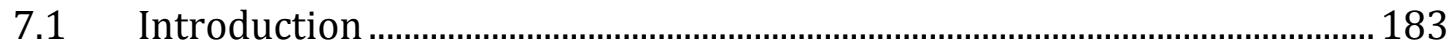

7.1.1 Technology Acceptance Model...................................................................... 183

7.1.1.1 Perceived Usefulness........................................................................... 184

7.1.1.2 Perceived Ease of Use.......................................................................... 184

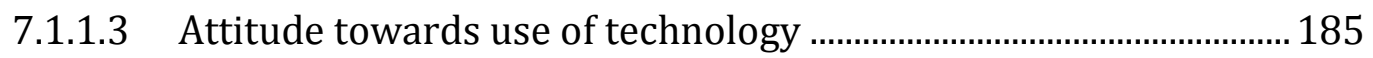

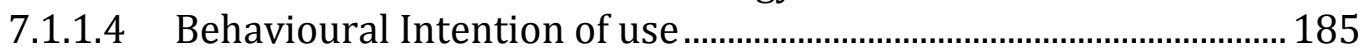

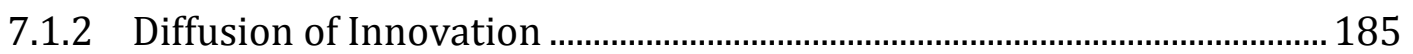

7.1.2.1 Individual leader characteristics ..................................................... 185

7.1.2.2 Internal characteristics of organisational structure ....................... 185

7.1.2.3 External characteristics of the organisation .................................... 186

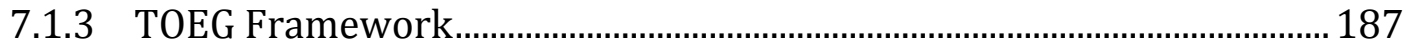

7.2 Embedding TOEG framework using original TOE framework .................... 188

7.2.1 Technology Context..................................................................................... 189

7.2.1.1 E-Readiness....................................................................................... 189

7.2.1.2 ICT resources underutilisation........................................................... 190

7.2.1.3 Perceived Benefits of using ICT in higher education ....................... 191

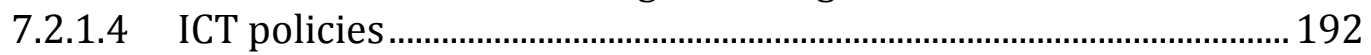

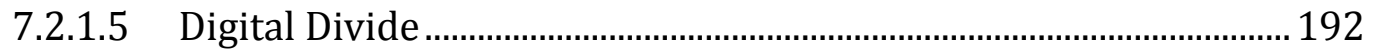

7.2.1.6 ICT confidence among teachers ....................................................... 192

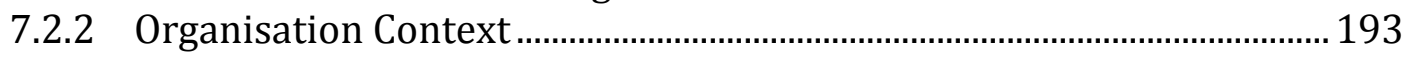

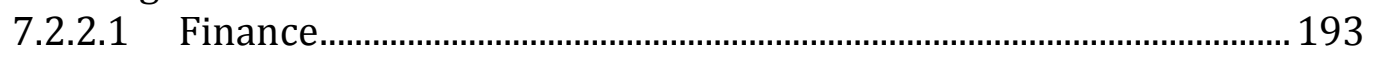

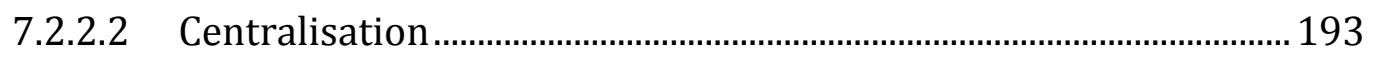

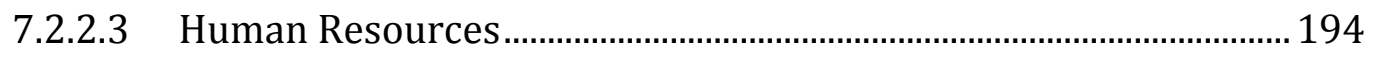

7.2.2.4 Top Management Support...................................................................... 195

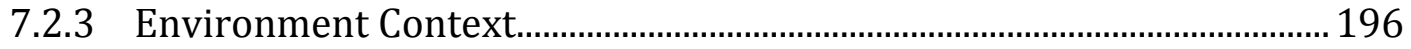

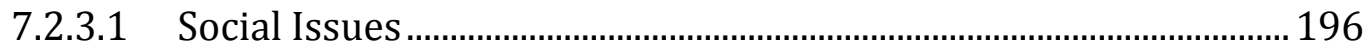

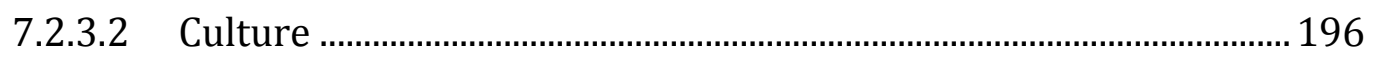

7.2.3.3 Pedagogical practices ........................................................................... 197

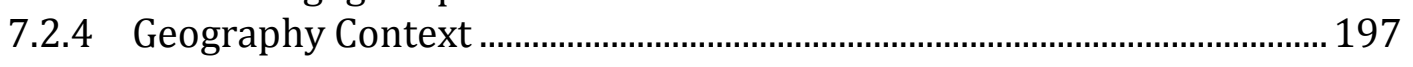




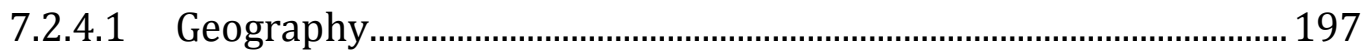

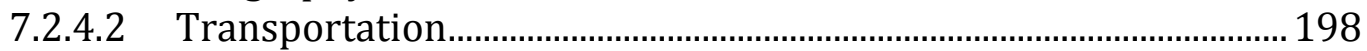

7.3 Extended TAM of ICT use in higher education in SIDS model ..................... 199

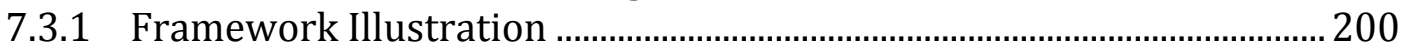

7.3.2 Technology-Organisation-Environment-Geography (TOEG) framework 201

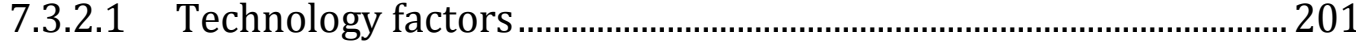

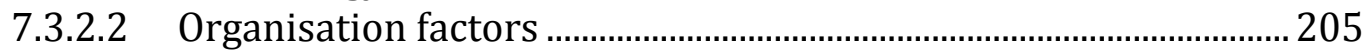

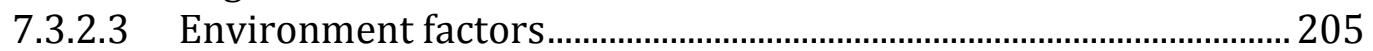

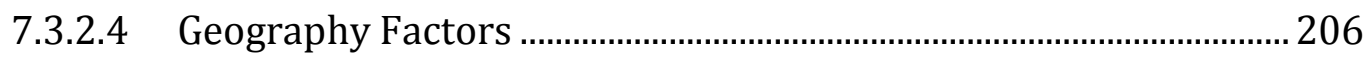

7.3.2.5 Linkages between TOEG and DOI factors........................................... 207

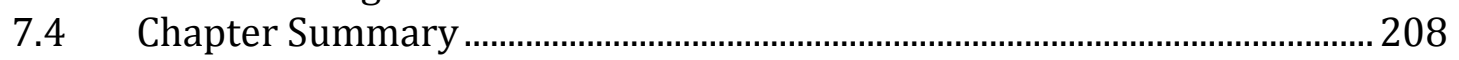

8 DISCUSSION

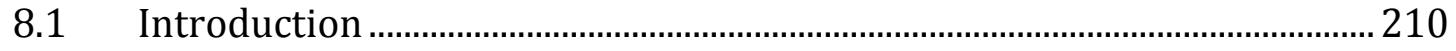

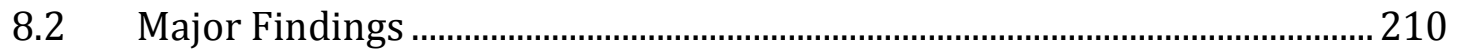

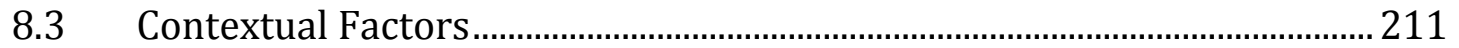

8.3.1 Technology Context Discussion................................................................... 212

8.3.2 Organisation Context Discussion ............................................................... 220

8.3.3 Environment Context Discussion................................................................. 226

8.3.4 Geography Context Discussion ............................................................. 232

8.4 Practical roadmap, grounding UNESCO's Continuum Model of ICT development with TOEG framework...................................................................... 236

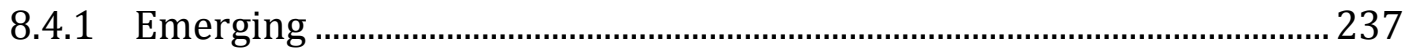

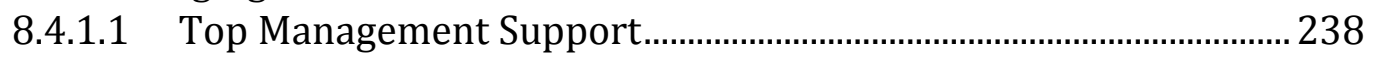

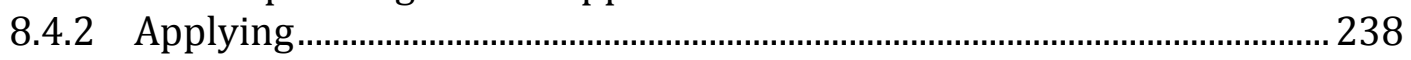

8.4.2.1 Motivating and creating ICT awareness among teachers .............. 238

8.4.2.2 Demonstrate the potential use of ICT ............................................... 240

8.4.3 Infusing ........................................................................................................ 241

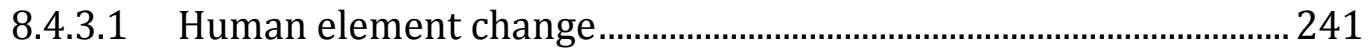

8.4.3.2 Foster an ICT culture within higher education ................................ 242

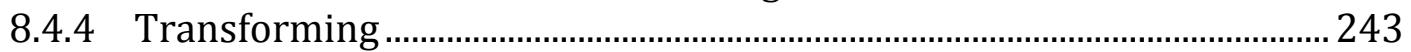

8.4.4.1 Systematically incorporating ICT into the higher education........ 243

8.4.4.2 Integrating three-tiered approach for ICT adoption in higher

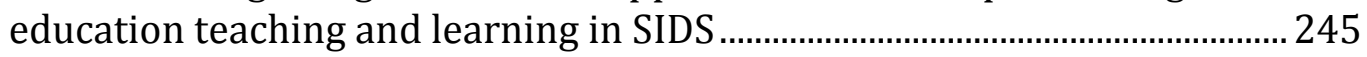

8.5 Adding value to research context among SIDS ............................................. 250

8.6 Adding value to research context in the Maldives .......................................250

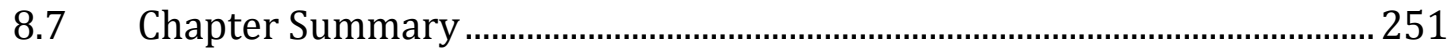

9 CONCLUSION

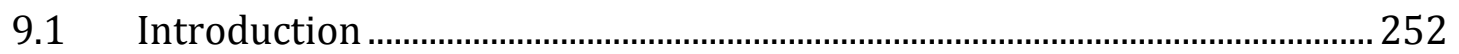

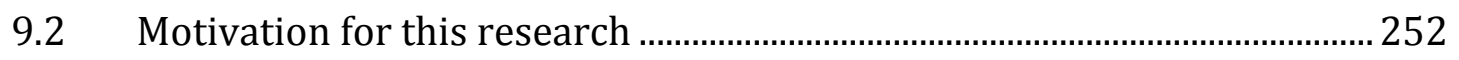

9.3 Answering research questions .................................................................... 252

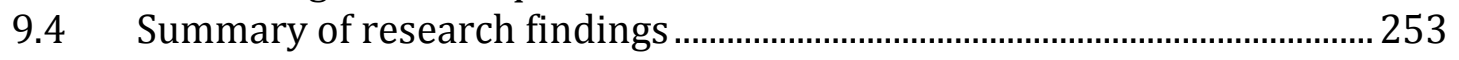

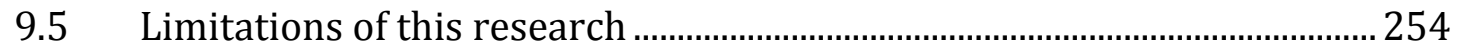

9.6 Generalisation of findings to other SIDS ..................................................... 255

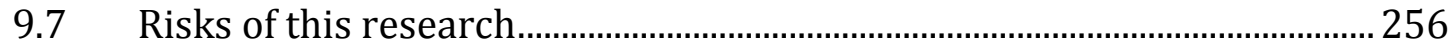

9.8 Contributions of this study .............................................................................. 257

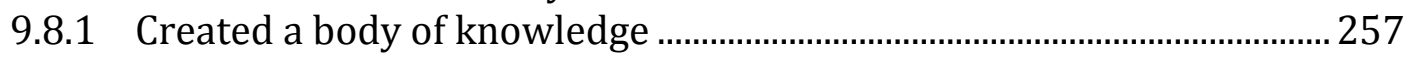


9.8.2 Awareness for utilisation of existing ICT in the Maldives

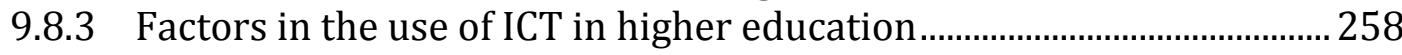

9.8.4 Contribution to the higher education sector ............................................. 258

9.8.5 How can ICT be used in higher education sector....................................... 258

9.8.6 Novelty of developed frameworks and models ....................................... 258

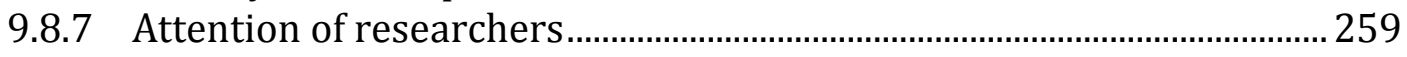

9.9 Implications of the research for SIDS in general ......................................... 259

9.9.1 Research Implications for Ministries of Education .................................. 260

9.9.2 Research Implications for Higher Education Institutes ........................... 260

9.10 Implications of the research for the Maldives .............................................. 260

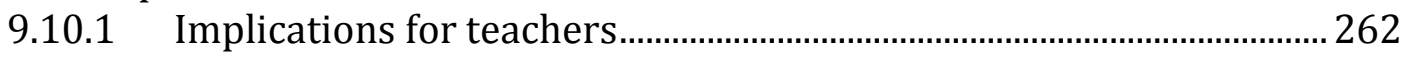

9.10.2 Implications for academic managers..................................................... 262

9.10.3 Implications for higher education institutes .........................................2. 263

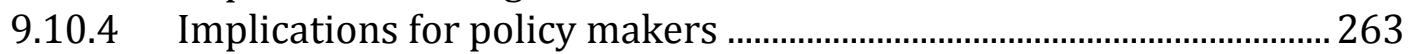

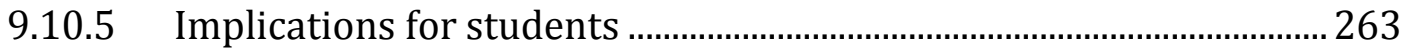

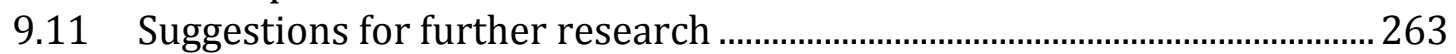

9.12 Final Comments ........................................................................................... 265

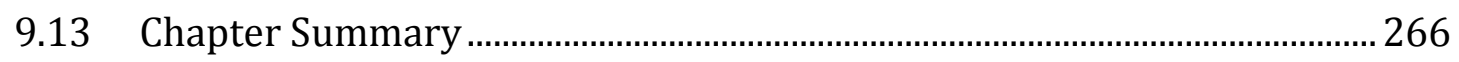

10 REFERENCES

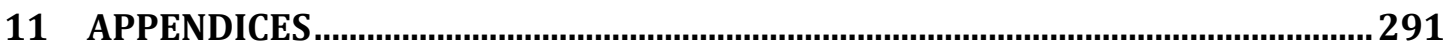




\section{CHAPTER 1}

\section{INTRODUCTION}

The first chapter gives the background of the research, statement of the problem and research questions. It also provides the research objectives and research significance. This chapter documents an understanding of research within the Small Island Developing States (SIDS) context, its theoretical underpinnings and contributions to knowledge. The chapter describes the structure of the thesis with a short description of each chapter. The definitions of key terms are also provided to capture the main components of this research. Finally, the chapter concludes with a chapter summary.

\subsection{Research Background}

Small Island Developing States (SIDS) are scattered unevenly over various remote islands and have small populations which makes it difficult to provide higher education services to their widely dispersed island populations. Information and Communication Technology (ICT) is an important tool in providing higher education to distant islanders.

This research investigates the importance of specific factors affecting the adoption of ICT in higher education in teaching and learning within SIDS, in the context of the Maldives as a case study. These factors appeared as significant outcomes of the research and elements for ICT adoption at a national and an institutional level.

Knowing these factors is vital and provides a gateway to know how ICT can be utilised within the higher education sector with respect to teaching and learning. The use of new technology in higher education is globally recognised as both a necessity and an opportunity. Information and Communications Technology can also make higher education accessible to many more students who are living on remote islands of SIDS. 


\subsection{Statement of the problem}

\section{Geography}

Small Island Developing States comprise 52 countries listed by The United Nations having small populations and remote islands with fragile environments (Gaible, 2009a; Gaible, 2009b; Santos-Paulino, 2011). Small Island Developing States tend to share related challenges, including limited resources and low populations (Atchoarena, Graca \& Marquez, 2008). Due to this geography, SIDS' growth is held back by high transportation costs, and little opportunity to create economies of scale (Gaible, 2009a). The greatest challenge for most SIDS is having many islands spread over a wide geographical area.

\section{Lack of higher education services in remote islands}

In many SIDS there is a lack of higher education services at the remote island level but reasonable services in the urban areas. The small populations in many islands are not conducive to achieving economies of scale in higher education and ICT infrastructures in remote islands, which increases the unit costs and human resource requirements. This creates more demand on higher education institutes in terms of administrative and technical operational difficulties.

Delivery of higher education services to distance locations becomes expensive. Due to limited human resources it is difficult to deploy skilled personnel in all distant locations. The lack of higher education services at island level is a major challenge faced by SIDS' governments.

\section{The challenge of utilising existing ICT resources}

Information and Communications Technology can play a fundamental role within the higher education sector of SIDS. With the geographical challenges, ICT can provide higher education to remote islanders with an easily accessible and learning experience. It is important to explore how ICT can be utilised in higher education. SIDS can benefit from taking advantage of the global information network to strengthen their higher education sector and utilise the existing ICT resources. Information and Communications Technology gives access to global 
resources (e-journals, databases, etc), which will strengthen higher education overall.

\section{Lack of ICT skilled people}

The lack of ICT skills and human capital has been identified as one of the main obstacles to successful adoption, management, or use of IT (Adam \& Urquhart, 2007). Kozma (2005) argued that education and development of human capital should be central to the development strategies of a country. The lack of skilled people is a major problem in SIDS. The development of human capital is important in the national context in order to deploy higher education services to remote islands.

\section{Lack of scholarly research}

Globally, there is a lack of research addressing the adoption of ICT in higher education within the specific context of SIDS. Walsham and Sahay (2006) stated that they would like to see more geographical research from developing countries in order to make academic contributions to important problem areas (see also Walsham, Roby \& Sahay, 2007). This research will make a contribution in this respect.

\section{Lack of theories and frameworks}

There is a lack of SIDS related theories or frameworks for the adoption of ICT in the higher education sector. Sound models and frameworks are needed to assist the implementation of any ICT-enabled technologies within the higher education sector of SIDS.

\section{Fulfilment of goals and policy documents}

The use of ICT in the higher education sector is part of the Seventh National Development Plan or Vision 2020 objectives to fulfil the educational goals of the Millennium Development Goals of the United Nations (Cambridge Education, 2005; Department of National Planning, 2011). 
On education, Vision 2020 stated: "An educated, knowledgeable and skilled citizenry is essential for making Vision 2020 a reality. Developing the competence of the people will be therefore a top priority in shaping the desired course for the future that embraces the aspirations of the nation" (Cambridge Education, 2005, p.43). Vision 2020 is targeted to make the Maldives a top-ranking nation among middle-income developing countries by 2020 (Ibrahim, 2006).

\subsection{Research Questions}

This research asks two questions.

\section{Primary Research Questions:}

1. What are the factors that influence the adoption of ICT within higher education teaching and learning in SIDS such as the Maldives?

2. How can ICT be used to address the issues within the higher education teaching and learning of the SIDS such as the Maldives?

In order to answer the two research questions, the underlying assumption is that ICT is treated as one of the influential factors. It is assumed that ICT should play an important role in delivering higher education services to remote islands. The delivery of higher education is dependent on a number of factors: technological, environmental, organisational and geographical. This research concentrates on the adoption of ICT, and its role in higher education teaching and learning in SIDS such as the Maldives.

\subsection{Research Objectives}

The following objectives are formulated for this research:

1. To explore the factors that influence ICT use in the higher education teaching and learning in SIDS such as the Maldives.

2. To find out how ICT can be used to address the issues within the higher education teaching and learning of the SIDS. 
3. To develop a TOEG framework based on the original TOE framework.

4. To develop a model for ICT adoption within higher education in SIDS using information system theories and frameworks.

5. To determine the practical implication of using ICT in higher education based on United Nations Educational, Scientific and Cultural Organization (UNESCO) continuum model of ICT development with TOEG framework, which will create a practical roadmap for higher education policy makers.

\subsection{Research Significance}

ICT is increasingly used in higher education institutes and is established in theoretical and professional practice. Research in this area is critical to gain a wider theoretical understanding of the future directions of ICT in higher education. There were gaps in the research literature, particularly with respect to the use of ICT in higher education in the context of SIDS such as the Maldives.

The researcher was confident that this study would fill some of these gaps, expand knowledge relevant in the context of SIDS and stimulate debate on related topics. It is an important phenomenon for researchers, professionals and decision makers. The findings and conclusions of this study are useful for policy makers at national and international levels.

\subsection{Research in SIDS context}

There has been very little research undertaken on ICT use in higher education in the context of SIDS. This research is a vital piece of documentation to assist stakeholders in the higher education sector within SIDS. The researcher believes that work of this nature would help the Maldives and offer the possibility of replication in other SIDS. Research carried out in the context of SIDS with an emphasis on ICT in higher education teaching and learning is scarce. 


\subsection{Theoretical underpinnings}

A theoretical model of ICT adoption in higher education in SIDS is necessary due to the lack of research. The need for such a model is important for academics and decision makers within the higher education sector of SIDS.

The exploration of factors provides an understanding of how ICT is used in higher education in SIDS, leading to improved models of implementation of ICT. This research uses one information systems framework and two information system theories, a) Technology, Organisation and Environment (TOE) framework, b) Technology Acceptance Model (TAM), and c) Diffusion of Innovation (DOI). In addition UNESCO continuum model of ICT development to provide a practical roadmap for higher education sector.

\subsection{Contributions to knowledge}

This research attempts to add to the knowledge and practice of ICT-enabled higher education in SIDS with respect to technical, organisational, environmental and geographical factors. The study proposes a TOEG framework that is relevant to SIDS and can be applied to countries with similar contexts. In conclusion, the research is novel and contributes to knowledge through:

1. Identification of factors influencing the use of ICT in higher education.

2. Developing a TOEG framework based on the original TOE framework that provides a foundation for policy makers in higher education to review the existing initiatives and understanding the ICT adoption in higher education.

3. Developing an Extended TAM of ICT use in the higher education in SIDS model using the TOEG framework integrated with TAM and DOI.

4. Developing a practical roadmap by using UNESCO's continuum model for ICT development and the TOEG framework. 


\subsection{Structure of this thesis}

This thesis comprises the introduction; literature review; research methodology; the case of the Maldives; research procedure; research results; research analysis; discussion; and conclusion.

\section{Chapter One - Introduction}

Chapter one explains and outlines a general introduction about the research, research background, statement of the problem and research questions. The chapter continues with the research objectives and research significance. The research in the SIDS context and its theoretical underpinnings are also explained. The chapter concludes by outlining the contributions to knowledge and structure of the thesis.

\section{Chapter Two - Literature Review}

Chapter two sets the context for this study by examining the literature from the fields related to the research topic, which include ICT, higher education and SIDS. The chapter identifies the factors associated with using ICT in higher education in SIDS. The chapter explains the different ICT media and their teaching and learning aspects.

The opportunity is taken in this chapter to introduce the relevant theories of technology in relation to higher education and also the theoretical lens used to examine the phenomenon. This chapter introduces the Technology-OrganisationEnvironment (TOE) framework. The Technology Acceptance Model (TAM) is used as an information systems theory to predict information technology acceptance. In addition, Diffusion of Innovation (DOI) is used to find out how new ideas and technology spread through cultures. A clear justification is provided about why TOE, TAM and DOI were selected for this research.

UNESCO's continuum model of ICT development is chosen to provide a framework for technology adoption within higher education. The frameworks and theories chosen by the researcher contribute to new understandings or problem solutions in this research context. 
This chapter provides a conceptual model for this research by integrating theories. Finally, this chapter concludes by identifying the literature gaps in this research area.

\section{Chapter Three - Research Methodology}

Chapter three establishes the research framework and the methodological choices outlining the philosophical assumptions. This research used a qualitative approach and an interpretive paradigm, together with case research methodology. The chapter provides a linkage between the research paradigm and methodology used to answer the two research questions.

The chapter explains the different data collecting methods used in this research. The main data collection was primarily based on interviews, focus group discussions, qualitative survey and document reviewing. The chapter explains the measures taken to ensure the quality of qualitative research with respect to this study. The chapter also describes ethical considerations, confidentiality and anonymity, together with the triangulation approach used in this research.

\section{Chapter Four - Case of the Maldives}

Chapter four describes the specific case study of the Maldives, which was the location where the research was carried out. The chapter explains the background of the Maldives and its geography, economy, population, system of governance, culture, Islamic dimensions and gender aspects.

The chapter throws light on the education system and more specifically higher education in the Maldives. The chapter also explains the existing ICT

infrastructure and projects in the Maldives. Finally, the chapter makes a comparison between the Maldives and other SIDS and also with regional countries.

\section{Chapter Five - Research Procedure}

Chapter five highlights the data collection procedure and what really happened during the research. The chapter describes how the research sites were selected and data analysis. The chapter explains how the data was analysed, including 
translating, transcribing, and identification, coding and conceptualisation of themes. The chapter finishes by explaining the data collection experience and roles played by the researcher.

\section{Chapter Six - Results - Specific Factors Discovered}

Chapter six reveals the specific factors originating from the data that have bearing on the use of ICT in the higher education teaching and learning of SIDS such as the Maldives. The factors are categorised using the TOE framework. The TOE framework comprises technology, organisation and environment. This chapter finds an answer to the first research question of this study.

\section{Chapter Seven - Analysis - How ICT can be used in higher education}

Chapter seven describes the framework illustrating the ICT adoption process at the organisational and national levels. The revised TOE framework is introduced in this chapter by embedding the factors discovered in Chapter 6 to produce the Technology, Organisation, Environment and Geography (TOEG) framework. The geography is used as a fourth element for the TOEG framework.

This chapter uses the TOEG framework and thoroughly reviews each of the factors associated with the four elements (i.e. technology, organisation, environment and geography). These factors are based on the context of the Maldivian higher education sector. This chapter finds an answer to the second research question of this thesis. The chapter includes an Extended TAM of ICT use in higher education in SIDS model using the TOEG framework integrated with TAM and DOI.

\section{Chapter Eight - Discussion}

The first part of chapter eight demonstrates how the TOEG framework might be adopted in SIDS. The major findings are then compared with the literature review of this research. This chapter discusses whether the findings and results are consistent with or different from existing perspectives. The last part of the chapter uses UNESCO's continuum model for ICT development and the TOEG framework to formulate a practical roadmap of the research findings for the use of ICT in the provision of higher education in SIDS. 


\section{Chapter Nine - Conclusion}

Chapter nine presents a summary of the entire thesis. This chapter provides an overview of the research motivation and a summary of key findings. The concept of generalising findings to other SIDS, the risks of this research, and its contribution to knowledge and research novelty is explained. The limitations are outlined, together with the research explains the research implications for SIDS in general and in the context of the Maldives, in particular. The chapter concludes by providing practical suggestions for further research and final comments from the researcher.

\subsection{Definition of key terms}

Higher Education - Higher Education is post-secondary education at higher education colleges, or universities. In the context of the Maldives Qualification Authority (MQA) framework, higher education is defined as any qualification attained at or above the diploma standard. For example: diploma, advanced diploma, bachelor's degree, postgraduate degree and doctoral degree (MQA, 2009).

Information and Communication Technology (ICT) - ICT are technologies such as computers, the Internet, broadcasting technologies (radio and television) and telephony (Khan, Hasan \& Clement, 2012). In this research, ICT is used interchangeably with Information Technology (IT), a term used in the Maldives and around the world.

Small Island Developing States (SIDS) - a group of 52 small island developing countries facing similar problems such as social, economic and environmental vulnerabilities (UN-OHRLLS, 2011).

ICT for teaching: ICT for teaching are "those technologies that support the teacher in their teaching of students. This could include a teacher computer (laptop, desktop or other device) that can be connected to a data projector and sound system to provide rich media to the entire class" (Douglas, 2011, p.128). 
ICT for learning: ICT for learning "is characterised as being student-centric. It is ICT focused upon the needs of the student to continue their learning and as such this ICT needs not only to suit the learner but also to be available to the learner when they require it” (Douglas, 2011, p.129).

\subsection{Summary}

This chapter has presented the background to the subject of this research, problem statement and research questions. It outlined the research objectives, research significance, research within the SIDS context, theoretical underpinnings and contributions to knowledge. The chapter concluded with the structure of the remaining chapters and definitions of key terms. The next chapter focuses on a literature review of the areas that are relevant to this study. 


\section{CHAPTER 2}

\section{LITERATURE REVIEW}

"While education unlocks the door to development, increasingly it is information technologies that can unlock the door to education" Wims \& Lawler (2007, p.7)

\subsection{Introduction}

This chapter reviews the key elements of this research: ICT, higher education, and SIDS. These elements are explained by identifying the main characteristics and their interactions: SIDS and higher education; SIDS and ICT; and ICT and higher education. The concept of ICT as media is also explained in relation to its teaching and learning aspects.

The research selected two information systems theories and a theoretical framework as appropriate to this research. The two theories and the framework selected for this study are the Technology Acceptance Model (TAM) theory and Diffusion of Innovation (DOI) theory and the Technology Environment and Organisation (TOE) framework.

In addition, UNESCO's continuum model of ICT development was also selected as a practical roadmap for adopting ICT within the higher education context. Appropriate justifications will be provided to ensure the relevance of these information system theories and framework.

\subsection{ICT, SIDS and higher education}

ICT is utilised to a large extent in higher education and it has become an essential tool in teaching and learning. This literature review entails three main areas: ICT, SIDS, and higher education. Figure 1 represents the main concepts of this research showing the intersection of ICT and SIDS (i.e. how ICT might be used in SIDS); the intersection of ICT and higher education (i.e. how ICT might be used in higher education); and the intersection of SIDS and higher education (i.e. how higher education is practiced in SIDS). Finally, the intersection among SIDS, ICT and higher education provides the real subject of this research inquiry. 
The literature review covers relevant studies from the areas of SIDS, ICT and higher education, viewing these from methodological, theoretical and practical standpoints, and grounding them in the body of academic knowledge. This research is primarily focused on the context of SIDS, and, secondly, on the Maldives as a special case of SIDS.

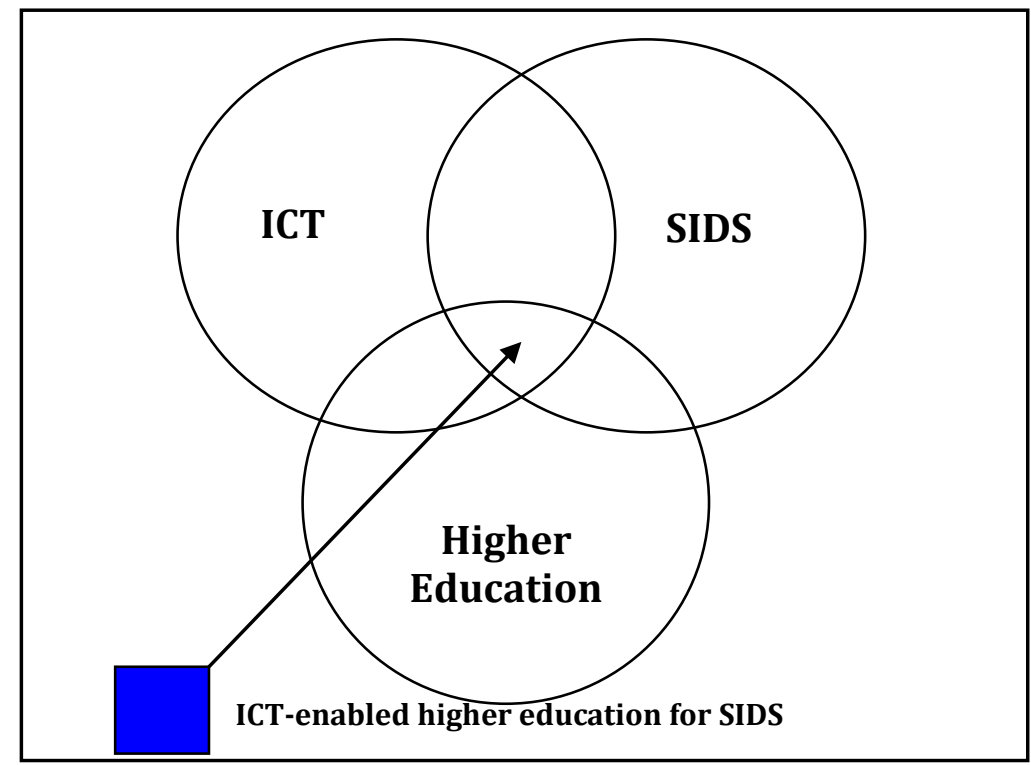

Figure 1: Subject of research inquiry

\subsubsection{Small Island Developing States (SIDS)}

Small Island Developing States share similar challenges: small growing populations, limited resources, remoteness, prone to natural disasters, and fragile environments (Atchoarena, Graca \& Marquez, 2008; Gaible, 2009a; Gaible, 2009b; Waheed; 2009). The populations of some SIDS tend to be concentrated in a few areas due to migration from remote areas to urban areas, with three out of four SIDS scattered unevenly among the different islands that make up a nation (Bacchus, 2008).

There are a number of definitions of "small" but the UNCTAD (2004b) report indicated that the main conceptual problem is how to define "small" utilising definitions such as population, national income, or the share of world trade (Gaible, 2009a). The most frequently used criterion for "small" has been a population threshold of 1.5 million (Von Tigerstrom, 2005), as proposed by the Commonwealth Secretariat and reflected in the report of the Commonwealth Secretariat/World Bank Joint Task Force (see World Bank, 2000). The best way to define SIDS is to select several geographic and economic criteria such as population, size and GDP 
(Atchoarena, Graca \& Marquez, 2008; UN-OHRLLS, 2011). Von Tigerstrom (2005) argued that some states with larger populations may also be included as SIDS on the basis that they share the relevant characteristics.

With regard to small populations Wong (2011) stated that SIDS may have "small population but high density and increasing pressure on limited land resources. In some cases, the resources are heavily stressed from unsustainable human activities" (p.2). In Fiji, internal migration resulted in 94\% of the nation's population living on the two main islands, while the remaining 6\% was distributed among the 97 small islands (Bacchus, 2008). SIDS face the challenge of less populated and poorer areas increasing the chance of urban migration (Bacchus, 2008).

Small Island Developing States display substantial diversity within each group of small states but also tend to share common features and challenges (UN-OHRLLS, 2011; Von Tigerstrom, 2005). Small Island Developing States face serious challenges with governance, communications and transportation (Gaible, 2009a; Koonjul, 2004) due to their smallness and economic situation. Small Island Developing States are a diversified group and include some islands that are not small (e.g. Papua New Guinea), not truly islands (e.g. Guyana), not developing (e.g. Bahrain), nor states (e.g. Netherland Antilles) (Wong, 2011). The limited population in SIDS means that they do not benefit very much from economies of scale (Bacchus, 2008).

Challenges such as limited resources, remoteness and susceptibility to natural disasters faced by SIDS are numerous and according to the World Bank (2000) there are various characteristics that cause development challenges to SIDS (UNOHRLLS, 2011; Gaible, 2009a; Von Tigerstrom, 2005). According to the World Bank (2000), Binger et al. (2002) and Von Tigerstrom (2005), these challenges are remoteness, natural disasters, environmental change, poverty and limited institutional capacity. The frequency of natural disasters among SIDS is the key aspect in explaining their vulnerability to environmental shocks (Santos-Paulino, 2011). 
Natural disasters such as tsunami are a fact of life in most SIDS, and cause considerable damage, and recovery periods from these disasters are long. In addition, due to limited resources, SIDS often have higher poverty levels than other developing countries, despite doing relatively well on some development indicators, such as education (Von Tigerstrom, 2005). The economic volatility and vulnerability of the population to poverty have significance impacts in SIDS (Santos-Paulino, 2011).

The issue of remoteness (i.e. the distances between islands and from other economies) as noted by the World Bank (2000) raises the question of transport within and between the islands of small island states. Despite all the vulnerable features of SIDS, Kirkman (2002) also noted positive points: "Relative to other developing countries, SIDS have exhibited strong ICT growth, especially over the past five years, in some cases surpassing average levels of key Networked Readiness variables for developing countries as a whole" (p.1). Nevertheless, SIDS share common challenges and face a greater risk of marginalisation from global economic activities than other developing countries (Santos-Paulino, 2011).

\subsubsection{ICT in SIDS}

Information and Communications Technology is significant for SIDS, because of their smallness and isolation from the major centres of production and consumption (UNESCO, 2011). The role of ICT offers numerous opportunities for progress in all aspects of life and benefits to social services and education in SIDS (Sealy, 2003). Information and Communications Technology services within SIDS tend to be fairly small and telecommunication services are limited (Farrell \& Wachholz, 2003). Some SIDS (Cook Islands, Fiji, Kiribati, Micronesia, Nauru, Niue, Samoa and Tonga) have limited ICT infrastructure but need the adaptation and application of sound technologies to counter the effect of vulnerability (Binger et al., 2002). Information and Communications Technology has a role to play in developing education in SIDS (Sealy, 2003).

Similar kinds of ICT infrastructure exist, for example, in the Indian Ocean island state of Mauritius, which has introduced e-Learning in its tertiary studies in order 
to democratise, provide greater access, and capture a wide range of lifelong learners to better meet national and societal needs (Fagoonee, 2001). Mauritius has experienced good ICT growth in recent years and role of ICT in bridging the digital gap is acknowledged as important (Pee \& Kankanhalli, 2010). SchoolNet programmes funded by donor organisations are taking place in some SIDS and are a good example of how ICT is spreading the education system (Farrell \& Wachholz, 2003).

Although all SIDS tend to have some ICT infrastructure, a more general problem is the lack of ICT-guided policy (Loxley \& Jullien, 2004), with the exception of Singapore, which has well-established ICT policies in place. Some SIDS (Fiji, Papua New Guinea, Tonga and Tuvalu) face high initial costs of setup, lack of technical support and inadequate network infrastructure (Gaible, 2009a). According to Farrell and Wachholz (2003), SIDS countries with common problems with ICT resourcing, access and skills are as follows:

1. Lack of infrastructure (Cook Islands, Fiji, Kiribati, Micronesia, Nauru, Niue, Samoa and Tonga)

2. High cost of hardware (Fiji, Papua New Guinea, Tonga and Tuvalu).

3. Lack of knowledge and skills (Nauru, Papua New Guinea and The Maldives)

\subsubsection{SIDS in higher education}

Atchoarena et al. (2008) stated: "SIDS must find innovative strategies to expand access and participation at the secondary and tertiary levels" (p.167). The higher education plans for SIDS must carefully target new ways of providing educational services such as collaboration within tertiary institutions and should develop the requisite human resources for economic resilience (Binger et al., 2002).

Higher education is now seen as a legitimate right in SIDS rather than a privilege (Fagoonee, 2001). All SIDS have educational and training institutions, but only some have universities and higher education institutes (Binger et al., 2002; Farrell \& Wachholz, 2003). Nevertheless, higher education in SIDS has a role to play due to the worldwide trend for mass education that has already reached the shores of 
SIDS and growing community expectations for tertiary education (Fagoonee, 2001).

Small Island Developing States are trying to extend higher education by experimenting with new learning techniques such as blended learning in some places (Gaible, 2009a). Binger et al.'s (2002) report emphasised that the educational institutions within SIDS should change so that the principal tertiary institutions become demand-driven.

\subsubsection{Information and Communication Technologies (ICT)}

There are many definitions for ICT and the most elaborate version by Blurton (1999, p.1) defined ICT as the: “... diverse set of technological tools and resources used to communicate, and to create, disseminate, store, and manage information. These technologies include computers, the Internet, broadcasting technologies (radio and television), and telephony". Information and Communication Technologies are a networked system comprising data processing and storage and retrieval of information (Herselman \& Britton, 2002).

It is true that the use of ICT has now become an integral part of daily life for a large percentage of people in both developed and developing countries (Kirkwood \& Price, 2006). Kozma (2002) believed that ICT is becoming the heart of preparing students and teachers for participation in the teaching and learning society. Punie, Zinnbauer and Cabrera (2006) argued that "it is difficult and maybe even impossible to imagine future learning environments that are not supported, in one way or another, by ICT" (p.5).

Information and Communications Technology is not a cure for all educational dilemmas, even though today technologies are obligatory tools (Guri-Rosenblit, 2006; Jung, 2005). Information and Communications Technology when effectively incorporated into teaching and learning ensures interaction between learners and teachers, thus advancing cognitive skills development (Jones \& Cress, 2001; Punie et al., 2006). 
Information and Communications Technology has provided the tools needed to deliver education to remote geographical regions (Guttman, 2003). The integration of ICT is extensive, when looking at its existing widespread diffusion, especially among the young generation (Punie et al., 2006). Many researchers believed that ICT creates three important milestones: a) access to teaching and learning opportunities; b) improving the quality of learning and teaching; and c) delivering lifelong learning for adults (Bates, 2000b; Hefzallah, 2004; Oh, 2003; Tinio, 2000).

Information and Communications Technology in higher education is significant and includes satellite communications and the Internet, which are a revolution in distance learning, offering flexible learning opportunities (Guttman, 2003). Blurton (1999) explained the virtual learning concept as communities within which learning groups are based on shared purposes. In this way, ICT will have a significant impact everywhere in the world with respect to education (Herselman \& Britton, 2002).

\subsubsection{Higher Education}

The basic right of access to higher education is preserved in a number of international human rights instruments. The UN International Covenant on Economic, Social and Cultural Rights of 1966 declared, in Article 13, that "higher education shall be made equally accessible to all, on the basis of capacity, by every appropriate means, and in particular by the progressive introduction of free education" (OHCHR, 2007, p.5). However, many SIDS lag behind in their tertiary education development (UNESCO, 2011).

According to Teichler (2006), the word "higher" in the context of higher education means attaining specific quality with substantial knowledge. Accessibility, capacity and free education seem to be problems for institutions. Tertiary institutes are struggling with how to deal with the enormous challenges and opportunities posed by ICT (Breen, Lindsay, Jenkins \& Smith, 2001). As stressed by Microsoft ${ }^{\circledR}$ Scholar Mr. Edward Barboni: “... Integration of information technology into learning in higher education is an urgent priority. The higher education community must 
continue to find innovative ways to empower educators to use technology to enhance learning and prepare students for careers and a lifetime of learning" (Microsoft ${ }^{\circledR}$, 1997, p.1).

Higher education and tertiary education are interchangeable words used within the education system (Teichler, 2006). The revolution of learning and teaching necessitates reorganisation of the ways universities and higher education institutes are planned, managed and organised (Sife, Lwoga \& Sanga, 2007). The higher education sector has been undergoing a revolutionary change (Sife et al., 2007) in the past decade, driven by the Internet and e-learning technologies (Guttman, 2003).

Sife et al. (2007) expressed concern that many institutions fail to incorporate ICT into teaching and learning because they are using ICT to duplicate their traditional practices, content and control. The higher education sector exists within an environment marked by significant transformation, not only in education but also in the wider society (Kirkwood \& Price, 2006). The rate of new technological change must keep pace with the higher education system in terms of enhancements in knowledge and skills to meet the demands (Hong \& Songan, 2011).

The transformation in higher education is boosted with the use of ICT, which thus enhances the quality of education (Kozma, 2005; Sangrà \& González-Sanmamed, 2010; Tinio, 2000). In the same vein Tinio (2000) also pointed out that ICTs can enhance the quality of education (as also noted by Sangrà \& González-Sanmamed, 2010) by increasing learner motivation, engagement and acquisition of basic skills. There is increasing pressure in developing countries where higher education is held back by serious challenges to ensure that technological possibilities are viewed in the context of educational needs (Jaffer et al., 2007).

\subsubsection{ICT in Higher Education}

The use of technology in higher education is an important development. Wims and Lawler (2007) stated that "while education unlocks the door to development, 
increasingly it is information technologies that can unlock the door to education" (p.7). Technology has been rapidly developing and influencing society and daily life. The importance of education was emphasised by Guttman (2003) as: "education is first and foremost a fundamental human right, spelt out in Article 26 of the Universal Declaration of Human Rights (1948), which declares that "elementary" education shall be free and compulsory, and that higher levels of education will be equally available on the basis of merit" (p.13).

The developed and developing countries in the world are investing a great deal of funds in higher education through the use of ICT. Information and Communication Technology investment thus represents an opportunity for developing countries to significantly improve their higher education sectors. Tinio (2000) stated: ICTs greatly facilitate the acquisition and absorption of knowledge, offering developing countries unprecedented opportunities to enhance educational systems, improve policy formulation and execution, and widen the range of opportunities for business and the poor. One of the greatest hardships endured by the poor, and by many others, who live in the poorest countries, is their sense of isolation. The new communications technologies promise to reduce that sense of isolation and to open access to knowledge in ways unimaginable not long ago (p.6).

Modern trends suggest that there will be changes in higher education due to the abundance of opportunities through utilisation of ICT (Oliver, 2002). Technology may replace some aspects of teaching and learning, and can enhance or facilitate communication between teachers and students.

The World Declaration on Higher Education 1998 for the Twenty-First Century stated that: "Higher education institutions should lead in drawing on the advantages and potential of new information and communication technologies, ensuring quality and maintaining high standards for education practices and outcomes in a spirit of openness, equity and international co-operation" - Article 12 (cited in Guttman, 2003, p.66). 
Information and Communications Technology has the potential to change the social structure and operation procedures, and force educational institutions to react and change as well (Kozma, 2002; Oh, 2003). Stensaker, Maasen, Borgan, Oftebro and Karseth (2007) pointed out that global use of ICT in higher education has made a remarkable "big leap forward" from the early 1990s (also see Oh, 2003). Due to the diverse nature of student requirements and their circumstances, educational institutions need to be constantly reflecting on the changing higher education environment (Kirkwood \& Price, 2006).

Pedagogical and socio-economic factors have forced tertiary education to adopt and integrate ICTs that allow greater information accessibility, communication, cost-effectiveness, collaboration and teacher delivery (Sife et al., 2007; Wang, 2008). Several studies have confirmed that ICTs can play an important role in allowing teaching and learning anywhere and anytime (Guttman, 2003; Hefzallah, 2004; Punie, 2007; UNCTAD, 2004a). Guttman (2003) argued that ICT will be an enormously powerful tool for educational change within tertiary institutes in future.

Use of ICT is not a panacea for all the challenges faced by the higher education sector but it does influence traditional teaching and learning (Jaffer et al., 2007). ICT is becoming ubiquitous within higher education for enhancing teaching and learning (Balasubramanian et al., 2009; Jaffer et al., 2007).

Integration of ICT in higher education is also moving ahead in terms of personal computers for students, mobile technology, virtual worlds and other equally accessible and efficient forms (Hong \& Songan, 2011). Mobile telephony has advanced rapidly in SIDs, with greater mobile connectivity than PC-based connectivity (UNESCO, 2011). Higher Educational Institutes (HEI) should take the necessary steps to ensure proper mechanisms are in place to allow such forms of ICT to be integrated within their teaching and learning. 


\subsection{Information and Communications Technology as media}

The increased dispersion of ICT-enabled higher education can overcome the distance barriers to collaboration and knowledge sharing among geographically distributed students and teachers within the context of SIDS such as the Maldives.

Students use a variety of ICT tools in their academic life for word processing and presentations (Kozma \& Wagner, 2008). Technological components become better known in a technology-enhanced environment, as many activities are conducted through the support of an ICT tool (Wang, 2008). Information and Communication Technology can be used to connect learning to real world contexts: for example the use of video case studies to solve a life-like problem (Kozma \& Wagner, 2008).

\subsubsection{ICTs as Communication Media}

Communications are at the cornerstone of educational process and ICT can be used as a tool to remove communication barriers such as time and location. ICT use in education can be expected to rise dramatically due to the digital tools and applications available in digital form (Blurton, 1999). However, communication and access to information are limited in the rural island communities of the Maldives. This contributes to isolation and missed opportunities and hinders access to knowledge and skills that could help people generate income, and lead healthier and more productive lives.

E-mail is a tool that can be used to communicate between teachers and students. The most important features of e-mail are that it is a widely-used service on the Internet and an asynchronous form of communication (Hefzallah, 2004). The adoption of e-mail in higher education culture is growing as institutes use it as a channel for student contact (Breen et al., 2001). Hefzallah (2004) stated that " $e$ mail may be used to enrich and to enhance the educational experience of students" (p.191).

The digital generation is making use of ICT tools such as social networking sites and podcasting outside the formal learning environments (Scutter, Stupans, Sawyer \& King, 2010; Visagie \& Villiers, 2010). Punie et al. (2006) stated the 
"learning with, for instance, mobile game technologies can indeed make learning more pleasant and more effective" (p.13).

\subsubsection{ICTs as Collaborative Media}

Information and Communications Technology enables high-level educational collaborations among students, teachers and groups of people. Blurton (1999) explained that these collaborations can take place between individuals in widely dispersed geographical locations. It is also helpful in the development of collaborative learning, where students can work together on common tasks in remote locations (Bates, 2000b). Wang (2008) stressed that the learning environment must offer appropriate tools so that students can easily communicate and collaborate with others. By utilising Web 2.0 tools such as blogging and tweeting students can share their ideas and work collaboratively with peers (Lai, 2011).

The role of the Internet should play an important part in the rise of collaboration. Bates (2001b) believed that the Internet offers opportunities for international, cross-cultural and collaborative learning. Jung (2005) emphasised the importance of international collaboration and networking in education and professional development. Collaborations can take place in many forms, such as off line and real-time chat systems (Blurton, 1999).

The use of computers also has an impact on the educational purpose, unlike conventional chalkboards, textbooks, radio and television (Aduwa-Ogiegbaen \& Iyamu, 2005; Hsu, 2007). Punie (2007) suggested that in a collaborative learning environment learners are more stimulated and motivated, thus they learn better. According to Wang (2008), social constructivists advocate collaborative learning where students can learn from each other, resulting in the construction of coherent knowledge.

Lai (2011) concluded that using digital technologies can improve the quality of the learning experiences to support collaboration. ICT-based systems are making it 
convenient to create positive attitudes towards a collaborative and constructive learning perspective (Sangrà \& González-Sanmamed, 2010).

\subsubsection{ICTs as Constructive Media}

Hsu (2007) described Wikis and weblogs or Blogs as "constructivist tools", which allow users to develop and maintain their own content. Wikis and Blogs are moderately new technologies for users. Blogs are becoming a vital source of information for Internet users (Hsu, 2007; Punie, 2007).

Blogs are essential educational tools, which offer the benefits of increased information sharing and publication of information (Hsu, 2007). However, Hsu (2007) argued that Wikis are easier to create, which means they can be used for better collaboration compared to Blogs.

Podcasting is a method of publishing files to the Internet with no cost (Panday, 2009). Podcasting is considered to be another valuable tool in education this could be a driver for mobile learning (Scutter et al., 2010). Hsu (2007) explained that Podcasts are important for two reasons: 1) a mobile device such as an iPod makes a good medium to distribute education materials; 2) information can be easily accessed, which makes Podcasting suitable for learners. ICT offers tools that can significantly improve communication with peers and teachers.

\subsubsection{ICTs as Pedagogy Media}

Digital technology allows more interactive pedagogy by supporting online learning communities (Lai, 2011). Conventional libraries are no longer merely places for storing printed materials, and accessing various digital libraries is now the new practice in tertiary institutes (Hefzallah, 2004). These new online collections contain printed works like textbooks, journals, illustrations, maps, photographs, 3D models, animations and audio files (Blurton, 1999). These technological developments and the rise of online databanks are leading to an increase in learning outside the traditional classroom (Hefzallah, 2004). Students and teachers can use the Internet as an important informative tool for research, document searches and other academic work. 
Electronic and digital publications are often more up-to-date than printed information. Due to electronic publications, learners and teachers are increasingly able to access high quality documents at any time, at any place (Bates, 2000b). The World Wide Web (WWW) has the advantage that, through Internet links, teachers can access websites, and bring materials from these sites into online lectures (Bates, 2000b).

The use of presentational software such as Microsoft ${ }^{\circledR}$ PowerPoint is a simple example of computer technology to enhance lecture teaching (Bates, 2000b; Hsu, 2007). The employment of computing technologies includes the use of Microsoft ${ }^{\circledR}$ PowerPoint, web-based portals such as Blackboard and WebCT (Hsu, 2007) and video-conferencing (Bates, 2000b).

Video-conferencing is particularly popular in multi-campus organisations, such as state university systems in the USA (Bates, 2000b). A rural campus with few students can be linked to a larger class in the urban area without the need to hire a teacher (Bates, 2000b). Lai (2011) noted a lack of understanding among higher education institution teachers that why and how technology should be embedded in the traditional pedagogy. Nevertheless, teaching is changing with the help of new technologies and teachers need to be willing to utilise the new concepts such as video conferencing.

\subsection{Teaching and Learning Aspects}

Kozma (2002) claimed that education is at the centre of a learning society. This is due to the fact that higher education is facing unprecedented change as students and teachers are driven towards the knowledge society of the future (Jaffer et al., 2007; Sife et al., 2007). In a knowledge-driven society where information and knowledge are quickly increasing, it is vital for students to be able to discuss and analyse their learning activities within a social context (Punie, 2007).

Punie (2007) emphasised the importance of learning "main objective of learning is also important for its contribution to people's emancipation, empowerment and self- 
fulfilment and learning objectives such as social competence, critical thinking, and knowledge sharing and cooperation techniques also need to be pursued" (p.186).

\subsubsection{Constructivism}

Constructivism refers to "... set of psychological theories that share common assumptions about knowing and learning. Although constructivist theories have implications for pedagogy and instruction, they are not theories of either" (Swan, 2005, p.2). Constructivism can be considered as a major theory of learning, and in broader terms as a philosophy of education. Koohang, Riley and Smith (2009) defined constructivism as "... active construction of new knowledge based on a learner's prior experience" (p.92). The constructivist concept offers a powerful model for explaining how knowledge is produced and constructed from experience (Gordon, 2008).

Constructivist theories of learning assume that meaning is imposed by the individual because people construct new knowledge and understandings based on what they believe, prior experiences, and socio-cultural contexts (Dede, 2008). The fundamental difference between constructivism and objectivism in learning is that constructivism emphasises the construction of knowledge while objectivism concerns the object of knowing (Tam, 2000).

Prior knowledge plays a vital role in actively constructing knowledge, creating, inventing and developing one's own knowledge and meaning (Liu, 2010). Central to the principle of constructivism, learning is an active process (Koohang et al. 2009; Liu, 2010).

Distance learning provides a mechanism to infuse constructivist approaches, where students learn through self-motivation, self-direction and collaboration with each other (Mattar, 2010). Distance and flexible learning offer an effective route to widen access to higher education (UNESCO, 2011). The penetration of technology into the learning can have effects for learning, with students learning individually using technology for rich learning contexts, peer tutoring via computer, and telecommunications (Tam, 2000). There is a complementary relationship between 
computer technologies and constructivism with the implementation of each benefiting the other (Nanjappa \& Grant, 2003).

Constructivist approaches in teaching and learning originated from work of Jerome Bruner, Jean Piaget and Lev Vygotsky (Liu, 2010; Nanjappa \& Grant, 2003). Gordon (2008) advocated that in a constructivist classroom setting teacher and studentdirect learning is equal, requiring teachers to take an active role in the learning process. The teacher can act as facilitator whose role will be to help students become active participants in their learning in order to connect prior knowledge with new knowledge.

\subsubsection{Collaborative learning}

The use of collaborative learning using ICT encourages interaction among students, teachers, regardless of where they are. Collaboration enables learners to work with people from different cultures, thus enhancing learner and teacher collaboration and communication skills (Tinio, 2000). Teaching and Learning in a collaborative model shifts from knowledge transfer or discussion (the cooperative model) toward all participants sharing the construction of their knowledge (Clough, Jones, McAndrew \& Scanlon, 2008; Moore, 2005).

Information and Communications Technology allows for greater interactivity between students and teachers giving learners the opportunity to continue at their own pace using a combination of video, audio and text to improve the quality of instruction and learning (Guttman, 2003; Tinio, 2000).

The link between collaborative learning and ICT has revealed strong possibilities for a virtual environment (Sangrà \& González-Sanmamed, 2010). However, there are problems with collaborative learning in classrooms, and Liu, Wang, Liang, Chan, Ko and Jang (2003) pointed out two notable issues: processes of interaction may not be recorded, and high ability students tend to dominate the whole learning activity. 


\subsubsection{Student-Centred learning}

Bates (2000a) and Jones and Cresse (2001) pointed out that the student-centred approach is a constructivist approach to learning, which incorporates the needs of students in a way that is convenient, flexible and cost-effective and enables total control of learning.

Bates (2000a) also expressed that teachers will be more like "hired hands", guiding and at times originating new learning materials. The role of the teacher will change from knowledge provider to facilitator helping students to make choices and to solve problems (Guttman, 2003; Valentine \& Holloway, 1999; Webb \& Cox, 2004).

The student-centred concept was also evident in Erstad's (2003) study, where the learning environment for students was challenging and stimulating, which seemed to improve students' performance and learning processes (Schofield, 1997). Student-centred learning is always available for the students and is labelled as "personal", not teacher-directed (Douglas, 2011). The student-centered learning that puts students first contradicts the existing traditional teaching styles (i.e. teacher-centered) lecturing. There may be a conflict between the deep-rooted teacher-centred pedagogy and a shift to student-centred pedagogy with the introduction of ICT (Hu \& Webb, 2009).

Schofield (1997) believed that ICT can motivate students, increase peer interactions, and shift teachers' roles away from a didactic approach to studentcentred interaction. Erstad (2003) had no doubt that ICT can create new dimensions for closing the students' learning gap and being more active within the centre of their own learning, and more students could benefit from studentcentered learning. The student-centered approach ensures that learners work collaboratively, engage in meaningful contexts and develop problem-solving skills (Oliver, 2002). Kozma, McGhee, Quellmalz and Zalles (2004) agreed that the use of ICT in institutes is associated with student-centred and constructivist pedagogical practices. Douglas (2011) explained that, in order to be student-centric, the ICT device needs to be a "device of choice" such as an i-Pad or smart phone. 


\subsubsection{Distance Education}

There are many definitions of distance education but a generally accepted definition was provided by Godschalk and Lacey (2001) as: “... any approach to the delivery of education that replaces the same-time, face-to-face environment of the traditional classroom" (p. 476). The expressions distance education, electronic education, e-learning, online learning and virtual learning are terms that go handin-hand. The importance of technology education was significantly highlighted in the Maldives NDP under point 1.7 by the need to "develop guidelines for the establishment of online, virtual and distance education" (Department of National Planning, 2011, p. 136).

Distance education is an established teaching approach that has gained new momentum due to the development of information and telecommunicationsinteractive technologies beneficial to citizens' lifelong learning (Hefzallah, 2004). Distance education has grown fast and Sumner (2000) believed the two world wars encouraged the rapid growth and spread of distance education and its massive technological development.

The history of distance education had three main generations: the correspondence model; the multimedia model; and the tele-learning model (see Burgess \& Russell, 2003; Godschalk \& Lacey, 2001; Sumner, 2000). As Taylor (1994) and Burgess and Russell (2003) argued, there is now a fourth generation of distance education, the flexible learning model.

New communication technologies (i.e. the Internet) appear to offer possibilities for overcoming geographical access, resourcing, and cost barriers to learning (Gulati, 2008). The World Wide Web (WWW) has unique features that make it an excellent medium for communication (Hefzallah, 2004). The web-based system enables students and teachers to communicate at a distance through discussion forums, chats and e-mail to problem-solve and complete assignments (Godschalk \& Lacey, 2001). 
Information and Communications Technology in higher education now has ICT tools (i.e. hardware and software) that can solve some of the limitations such as lack of linkage between teachers and students separated by distance (Oh, 2003). WebCT and Blackboard are one of the mediums for posting courses online, offering discussion boards, mail systems, and live chat sessions, and content including web pages (Hefzallah, 2004; Kirkwood \& Price, 2006; Selwyn, 2007). Where there is a lack of opportunities in remote areas (Von Tigerstrom, 2005; World Bank, 2000), ICT can help to overcome the problems with geographical isolation, which is useful for teachers and students (Wims \& Lawler, 2007).

Table 1 shows the models of distance education as presented by Taylor.

\begin{tabular}{|c|c|c|c|c|c|}
\hline \multirow{3}{*}{$\begin{array}{l}\text { Models of distance education and } \\
\text { associated delivery technologies }\end{array}$} & \multicolumn{5}{|c|}{ Characteristics of Delivery Technologies } \\
\hline & \multicolumn{3}{|c|}{ Flexibility } & \multirow{2}{*}{\begin{tabular}{|c|} 
Highly \\
Refined \\
Materials
\end{tabular}} & \multirow{2}{*}{$\begin{array}{l}\text { Advanced } \\
\text { Interactive } \\
\text { Delivery }\end{array}$} \\
\hline & Time & Place & Pace & & \\
\hline $\begin{array}{l}\text { First Generation - The Correspondence Model } \\
\text { Print }\end{array}$ & Yes & Yes & Yes & Yes & No \\
\hline \begin{tabular}{|l|} 
Second Generation - The Multimedia Model \\
Print \\
Audiotape \\
Videotape \\
Computer-based learning (e.g. CML/CAL) \\
Interactive video (disk and tape) \\
\end{tabular} & $\begin{array}{l}\text { Yes } \\
\text { Yes } \\
\text { Yes } \\
\text { Yes } \\
\text { Yes }\end{array}$ & $\begin{array}{l}\text { Yes } \\
\text { Yes } \\
\text { Yes } \\
\text { Yes } \\
\text { Yes } \\
\end{array}$ & $\begin{array}{l}\text { Yes } \\
\text { Yes } \\
\text { Yes } \\
\text { Yes } \\
\text { Yes } \\
\end{array}$ & $\begin{array}{l}\text { Yes } \\
\text { Yes } \\
\text { Yes } \\
\text { Yes } \\
\text { Yes }\end{array}$ & $\begin{array}{l}\text { No } \\
\text { No } \\
\text { No } \\
\text { Yes } \\
\text { Yes } \\
\end{array}$ \\
\hline $\begin{array}{l}\text { Third Generation - The Tele-learning Model } \\
\text { Audio teleconferencing } \\
\text { Video conferencing } \\
\text { Audio-graphic (e.g. Smart 2000) } \\
\text { Broadcast TV/Radio + Audio teleconferencing } \\
\end{array}$ & $\begin{array}{l}\text { No } \\
\text { No } \\
\text { No } \\
\text { No }\end{array}$ & $\begin{array}{l}\text { No } \\
\text { No } \\
\text { No } \\
\text { No }\end{array}$ & $\begin{array}{l}\text { No } \\
\text { No } \\
\text { No } \\
\text { No }\end{array}$ & $\begin{array}{l}\text { No } \\
\text { No } \\
\text { Yes } \\
\text { Yes }\end{array}$ & $\begin{array}{l}\text { Yes } \\
\text { Yes } \\
\text { Yes } \\
\text { Yes }\end{array}$ \\
\hline $\begin{array}{l}\text { Fourth Generation - The Flexible Learning } \\
\text { Model } \\
\text { Interactive multimedia (IMM) } \\
\text { Computer mediated (Email, etc) }\end{array}$ & $\begin{array}{l}\text { Yes } \\
\text { Yes }\end{array}$ & $\begin{array}{l}\text { Yes } \\
\text { Yes }\end{array}$ & $\begin{array}{l}\text { Yes } \\
\text { Yes }\end{array}$ & $\begin{array}{l}\text { Yes } \\
\text { No }\end{array}$ & $\begin{array}{l}\text { Yes } \\
\text { Yes }\end{array}$ \\
\hline $\begin{array}{l}\text { Fifth Generation - Intelligent flexible learning } \\
\text { model }\end{array}$ & Yes & Yes & Yes & Yes & Yes \\
\hline
\end{tabular}

Table 1: Model of Distance Education (adapted from Taylor, 1994, p.2)

\section{a) Correspondence Model - First generation}

The correspondence model is based on print technology (Taylor, 2001). The earliest form of distance education was started in 1840 in England by Isaac Pitman to teach shorthand to aid business administration (Godschalk \& Lacey, 2001; Sumner, 2000). Sumner (2000) explained that, by the end of the nineteenth 
century, the correspondence model was well established in places like Canada and Australia.

\section{b) Multimedia Model - Second generation}

Godschalk and Lacey (2001) explained that the second generation of distance education started in the 1970s and still exists today. In short the multimedia model is based on print, audio and video technologies (Sumner, 2000; Taylor, 2001).

\section{c) Tele-learning Model - Third generation}

The tele-learning model is based on applications of telecommunications technologies for synchronous communication (Taylor, 2001). This model, sometimes called the multimedia model, started in the 1990s and is delivered through phone, satellite and cable lines (Godschalk \& Lacey, 2001). The third generation model commenced in the twentieth century, and started the information age with the help of the Internet, WWW and computer conferencing (Sumner, 2000).

\section{d) Flexible Learning Model - Fourth generation}

This model is based on online delivery via the Internet (Taylor, 2001). Most universities are now adjusting their infrastructure to accommodate this type of learning. The fourth generation distance education relies upon two-way communication via computer and technologies such as virtual reality (Burgess \& Russell, 2003).

\section{e) The Intelligent Flexible Learning Model - Fifth generation}

This model is the newest model in the series and has the potential to decrease the costs associated with providing access to online learning (Taylor, 2001). Taylor (2001) argued that the fifth generation is basically a derivation of the fourth generation, which aims to capture the features of the Internet and Web. Taylor (2001) also maintained that students will gain access to higher education at a cheaper price locally and globally with better quality education, more effective pedagogical and administrative services. 


\subsubsection{Electronic Learning}

Electronic Learning (e-Learning) refers to the use of ICT (i.e. computer system) to enhance and support learning (Sife et al., 2007) at all levels, both formal and nonformal, using an information network (Tinio, 2000). The principal technology being used for e-learning in the developed world is the Internet (Bates, 2001b).

E-learning offers uses for learners of all ages. Students enjoy its multi-media games and fun activities in acquiring very basic literacy skills; older students use its endless information resources for preparing homework, assignments and examinations (Guri-Rosenblit, 2006). E-learning is still at a premature stage when it comes to developing countries in particular, because they do not possess the appropriate resources and technology infrastructure to make e-learning available on a wide scale (Guri-Rosenblit, 2006).

\subsubsection{Blended Learning}

The term blended learning was defined as "... a system that combines face-to-face instruction with computer-mediated instructions" (Graham, 2005, p.5). A more comprehensive definition was provided by Watson (2008) as “... [combining] online delivery of educational content with the best features of classroom interaction and live instruction to personalize learning, allow thoughtful reflection, and differentiate instruction from student to student across a diverse group of learners" (p.4). Blended Learning combines online and face-to-face interactions, which includes providing online materials, and replacing portions of the face-to-face contents with electronic materials (Alebaikan \& Troudi, 2010).

Blended learning (face-to-face and online learning) has a significant influence on how students interact with their peers, and has transformed teaching and learning in and out of the traditional classroom (Benson et al., 2011). Benson et al. (2011) stated that there are challenges in implementing blended learning: “... build on positive staff attitudes, provide appropriate resources and help staff develop blends of teaching practices and use of technologies that deliver the best outcomes in particular teaching and learning contexts" (p.153). In addition, academic staff need 
to be aware of how to use the tools (multimedia, mobile technologies, Web 2.0 and social networks) in teaching (Benson et al., 2011).

Blended learning is most appropriate, according to Alebaikan and Troudi (2010), “... for students who live far away from the university or have other commitments that conflict with the on-campus class time” (p.51). The concept of blended learning is visualised to take the benefits of traditional teaching methods and online delivery (Alebaikan \& Troudi, 2010; Benson, Anderson \& Ooms, 2011). Graham (2005) explained that there are four reasons for choosing blended learning: pedagogy, access, flexibility, and cost-effectiveness. The pedagogy is different from the traditional face-to-face classroom since it requires a website for online interactions (Benson et al., 2011). The future of blended learning was explained by Watson (2008) as "likely to emerge as the predominant teaching model of the future" (p.4).

\subsubsection{Mobile Learning}

Mobile learning is a new phenomenon and its theoretical basis is currently under development (Kearney, Schuck, Burden \& Aubusson, 2012). Park (2011) defined mobile learning as "... the use of mobile or wireless devices for the purpose of learning while on the move” (p.79). A simple definition was provided by Kearney et al. (2012) as "... the occurrence of learning: the process of learning mediated by a mobile device" (p.2).

Mobile Learning (m-learning) is accessible from virtually anywhere (Liu et al., 2003). The concept of anytime and anywhere originated with online education but was revolutionised by mobile learning technologies (Caudill, 2007). The central advantage of learning with mobile technologies is the ability to learn within one's own context in time and space (Melhuish \& Falloon, 2010).

Mobile technologies such as mobile phones, iPods and iPhones, PDAs, and portable netbook computers have aroused considerable interest amongst the education sector (Melhuish \& Falloon, 2010). Mobile devices are becoming increasingly ubiquitous, and can be incorporated into teaching and learning (Park, 2011). 
Naismith, Lonsdale, Vavoula and Sharples (2004) noted that the challenge for educators in using mobile technologies is exploring how best to use the resources to support learning.

Mobility allows teaching and learning to extend to spaces beyond the traditional classroom, and within the classroom, mobile learning gives instructors and learners increased flexibility and new opportunities for interaction (Liu et al., 2003). Mobile technologies support learning experiences that are collaborative, accessible, and integrated with the world beyond the classroom.

Mobile Learning can use real-time video and audio streaming that allows urban and rural students to communicate with both lecturers and other students (Wataniya, 2011; Wataniya, 2012). The students have advantages with mLearning, such as greater ease of access and convenience, which streamline the learning process (Caudill, 2007).

Mobile technologies have a great influence on learning and are now becoming ubiquitous, with advanced functions for rich social connections, ICT awareness and Internet connectivity (Naismith et al., 2004). Research undertaken in the United Kingdom (UK) revealed that mobile technologies are a familiar part of the lives of most teachers and students (Naismith et al., 2004). The newest mobile phones and iPhones enable connectivity, either wired or wireless, anywhere and anytime (Dudding, 2009).

It is clear from the literature that there is a role for mobile learning in education. Mobile learning has three characteristics in pedagogy: authenticity, collaboration (Naismith et al., 2004), and personalisation (Cochrane, 2010). Cochrane (2010) also stated there is "... potential for mobile learning to bridge pedagogically designed learning contexts, facilitate learner-generated contexts, and content (both personal and collaborative), while providing personalisation and ubiquitous social connectedness, that sets it apart from more traditional learning environments" (p.134). 
Different researchers have said much the same thing; as Melhuish and Falloon (2010) noted there are five distinct features: a) Portability; b) Affordable and ubiquitous access; c) Situated, 'just-in-time' learning opportunities; d) Connection and convergence; e) Individualised and personalised experiences.

\subsection{Role of theory in research}

Creswell (2009) defined theory as “... interrelated set of constructs (or variables) formed into propositions or hypotheses that specify the relationship among variables (typically in terms of magnitude or direction" (p.51). According to Walsham (1995a), an important question for researchers is the role of theory in their research. Eisenhardt (1989) identified three distinct uses of theory in research: to guide a) guide to design and data collection; b) iterative process of data collection and analysis; and c) final outcome. A theory might be used in research as an argument, discussion or rationale to explain the phenomena (Creswell, 2009). This study is exploratory in nature and existing theoretical frameworks are important in order to understand about the existing knowledge and what the outcome will be.

The use of theory in interpretive cases research is important as it creates an initial theoretical framework, basis and approach on which initial empirical work is based (Walsham, 1995a). The choice of theory is essentially subjective and Walsham emphasised the freedom of choosing theories with which the researcher is comfortable (Walsham, 2006).

Walsham (1995a) warned not to use theory in a rigid way that might stifle new ideas and exploration. Therefore, it is necessary to maintain a considerable degree of openness to the collected data with changes to the initial assumptions and theories. The researcher is confident that choosing an existing theoretical framework will establish a leading edge, viewpoints, and a set of theoretical lenses through which to view the problem.

\subsubsection{Theories and frameworks}

Existing theories and frameworks developed by researchers have been used to explain the adoption and diffusion of technology. One of the most commonly 
employed models is the Technology Acceptance Model (TAM) developed by Davis (1989), which explains and predicts the acceptance of particular technologies across a range of populations (Lippert \& Govindarajulu, 2006).

In 1990 Tornatzky and Fleischer developed the Technology-OrganisationEnvironment (TOE) framework to explain the decision to adopt a technological innovation and its acceptance. As ICT-enabled higher education is an innovation, the TOE framework allows its adoption factors to be categorised.

Rogers' Diffusion of Innovation (DOI) also provides a theoretical lens to explain how an innovation diffuses through a society (Rogers, 2003).

\subsection{Technology-Organisation-Environment (TOE) framework}

The Technology-Organisation-Environment (TOE) framework (Figure 2) developed by Tornatzky and Fleischer (1990) has been a useful theoretical lens for understanding the technology adoption within organisations (Arpacl et al., 2012; Lippert \& Govindarajulu, 2006; Oliveira \& Martins, 2011; Pudjianto \& Hangjung, 2009). According to Pudjianto and Hangjung (2009), TOE provides a framework that explains the decision to adopt a technological innovation based on technological considerations, but also on the organisational and environmental contexts.

The TOE framework addresses the context in which innovation takes place, both within, and externally of the business. The TOE framework has a concrete theoretical basis, consistent empirical support and the potential to be applied to Information Systems innovation domains (Oliveira \& Martins, 2011). 


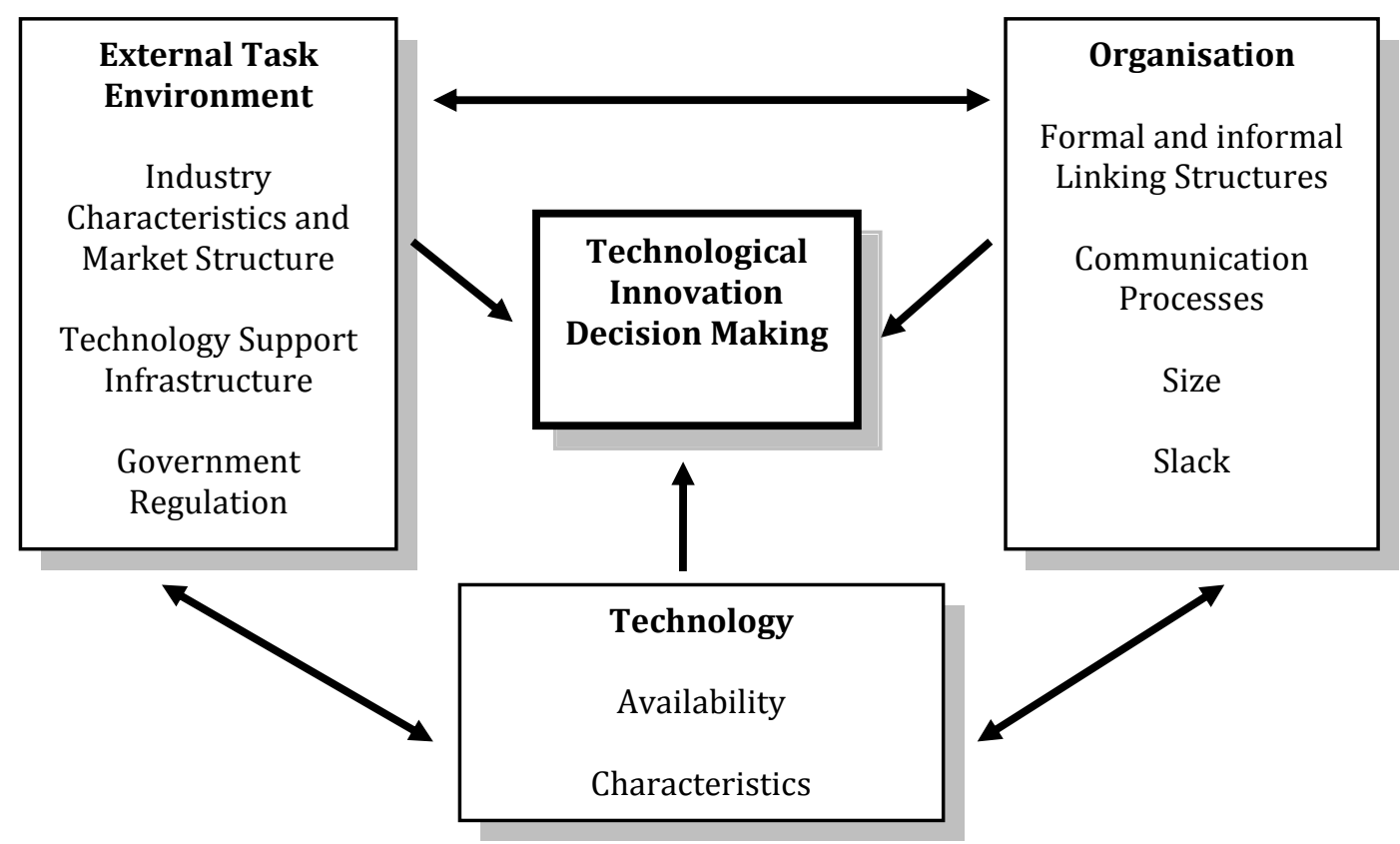

Figure 2: TOE framework (Source: Tornatzky \& Fleischer, 1990, p.154)

Several studies used the TOE framework as a theoretical lens to understand different IT adoptions, such as e-Government assimilation (Pudjianto \& Hangjung, 2009); e-Learning Implementation (Raouf, Naser \& Jassim, 2012); IT adoption at Firm Level (Oliveira \& Martins, 2011); e-Commerce (Awa \& Ukoha, 2012); OpenSystems (Awa \& Ukoha, 2012); Web Service Adoption (Lippert \& Govindarajulu, 2006); and mobile communications (Arpacl et al., 2012); and e-business (Hsu, Kraemer \& Dunkle, 2006). The IT adoption themes, research articles using TOE, research focuses and references are tabulated in Table 2.

\begin{tabular}{|l|l|l|l|}
\hline \multicolumn{1}{|c|}{ IT Adoption } & $\begin{array}{l}\text { Research articles } \\
\text { using TOE }\end{array}$ & \multicolumn{1}{|c|}{ Research Focus } & References \\
\hline $\begin{array}{l}\text { e-Government } \\
\text { Assimilation }\end{array}$ & $\begin{array}{l}\text { Factors affecting } \\
\text { the e-Government } \\
\text { Assimilation in } \\
\text { Developing } \\
\text { countries }\end{array}$ & $\begin{array}{l}\text { Technology: Existing technologies in use; } \\
\text { new technologies available } \\
\text { Organisation: organisational characteristics, } \\
\text { organisational readiness, top management } \\
\text { support } \\
\text { Environment: Regulatory environment and } \\
\text { competition environment }\end{array}$ & $\begin{array}{l}\text { Pudjianto \& } \\
\text { Hangjung } \\
(2009)\end{array}$ \\
\hline $\begin{array}{l}\text { e-Learning } \\
\text { Implementation }\end{array}$ & $\begin{array}{l}\text { Determinants of } \\
\text { E-Learning } \\
\text { Implementation } \\
\text { Success } \\
\text { In The Iraqi MoHE }\end{array}$ & $\begin{array}{l}\text { Technology: Information system } \\
\text { infrastructure, IS expertise } \\
\text { Organisation: Organisation compatibility } \\
\text { and organisation benefits } \\
\text { Environment: competitive pressure and } \\
\text { educational partner readiness }\end{array}$ & $\begin{array}{l}\text { Raouf, Naser } \\
\text { (2012) Jassim }\end{array}$ \\
\hline $\begin{array}{l}\text { IT adoption at } \\
\text { Firm Level }\end{array}$ & $\begin{array}{l}\text { Literature Review } \\
\text { of Information } \\
\text { Technology }\end{array}$ & $\begin{array}{l}\text { Technology: Internal and external } \\
\text { technologies such as includes current } \\
\text { practices and equipment internal to the firm }\end{array}$ & $\begin{array}{l}\text { Martins } \\
(2011)\end{array}$ \\
\hline
\end{tabular}




\begin{tabular}{|c|c|c|c|}
\hline & $\begin{array}{l}\text { Adoption Models } \\
\text { at Firm Level }\end{array}$ & $\begin{array}{l}\text { Organisation: scope, size, and managerial } \\
\text { structure. } \\
\text { Environment: industry, and competitors }\end{array}$ & \\
\hline e-Commerce & $\begin{array}{l}\text { Integrating TAM } \\
\text { and TOE } \\
\text { Frameworks and } \\
\text { Expanding their } \\
\text { Characteristic } \\
\text { Constructs for E- } \\
\text { Commerce } \\
\text { Adoption by SMEs }\end{array}$ & $\begin{array}{l}\text { Technology: perceived relative advantage } \\
\text { (gains), compatibility (both technical and } \\
\text { organisational), complexity (learning curve), } \\
\text { trialability (pilot test/experimentation), and } \\
\text { observability (visibility/imagination) } \\
\text { Organisation: firm's business scope, top } \\
\text { management support, organisational } \\
\text { culture, complexity of managerial structure } \\
\text { measured in terms of centralisation, } \\
\text { formalisation, and vertical differentiation, } \\
\text { the quality of human resource } \\
\text { Environment: Competitive pressure, trading } \\
\text { partners' readiness, socio-cultural issues, } \\
\text { government encouragement, and technology } \\
\text { support infrastructures }\end{array}$ & $\begin{array}{l}\text { Awa \& Ukoha } \\
\text { (2012) }\end{array}$ \\
\hline Open Systems & $\begin{array}{l}\text { Factors affecting } \\
\text { the adoption of } \\
\text { Open Systems: An } \\
\text { Exploratory Study }\end{array}$ & $\begin{array}{l}\text { Technology: Innovation such as perceived } \\
\text { Benefits; perceived barriers; perceived } \\
\text { Importance of compliance to standards, } \\
\text { interoperability, and Interconnectivity. } \\
\text { Organisation: complexity of IT } \\
\text { infrastructure; satisfaction with existing } \\
\text { systems; formalisation of system } \\
\text { development and management. } \\
\text { Environment: market uncertainty }\end{array}$ & $\begin{array}{l}\text { Chau \& Tam } \\
\text { (1997) }\end{array}$ \\
\hline $\begin{array}{l}\text { Web Service } \\
\text { Adoption }\end{array}$ & $\begin{array}{l}\text { Technological, } \\
\text { Organizational, } \\
\text { and Environment } \\
\text { Antecedents to } \\
\text { Web Services } \\
\text { Adoption }\end{array}$ & $\begin{array}{l}\text { Technology: Security concern, reliability } \\
\text { and deployability } \\
\text { Organisation: Firm size, firm scope, } \\
\text { technological knowledge and perceived } \\
\text { benefits. } \\
\text { Environment: Competitive pressure, } \\
\text { regulatory influence, dependent partner } \\
\text { readiness and trust in web service provider. }\end{array}$ & $\begin{array}{l}\text { Lippert \& } \\
\text { Govindarajulu } \\
(2006)\end{array}$ \\
\hline $\begin{array}{l}\text { Mobile } \\
\text { Communications }\end{array}$ & $\begin{array}{l}\text { Organizational } \\
\text { Adoption of } \\
\text { Mobile } \\
\text { Communication } \\
\text { Technologies }\end{array}$ & $\begin{array}{l}\text { Technology: relative advantage, } \\
\text { compatibility, complexity, visibility, result } \\
\text { demonstrability, trialability and cost. } \\
\text { Organisation: size, structure, innovativeness, } \\
\text { management support, resources and } \\
\text { information intensity. }\end{array}$ & $\begin{array}{l}\text { Arpacl, et al., } \\
\text { (2012) }\end{array}$ \\
\hline $\begin{array}{l}\text { E-Business } \\
\text { Diversity use: } \\
\text { diversity and } \\
\text { volume }\end{array}$ & $\begin{array}{l}\text { Determinants of } \\
\text { e-Business use in } \\
\text { U.S. firms }\end{array}$ & $\begin{array}{l}\text { Perceived benefits: perceived of innovations } \\
\text { Organisation readiness: firm, size, } \\
\text { technology resource, global level } \\
\text { External Pressure: trading partners } \\
\text { pressure, government pressure } \\
\text { Environment: regulatory conventions, } \\
\text { competition intensity } \\
\text { Control: industry effects }\end{array}$ & $\begin{array}{l}\text { Hsu, Kraemer } \\
\text { \& Dunkle } \\
(2006)\end{array}$ \\
\hline
\end{tabular}

Table 2: $\quad$ Some studies based on TOE framework

\subsubsection{TOE framework elements}

The TOE framework identifies three aspects that influence how an organisation adopts and implements technological innovations: Technology, Organisation, and Environment (Arpacl et al., 2012; Awa \& Ukoha, 2012; Chau \& Tam, 1997; Lippert 
\& Govindarajulu, 2006; Oliveira \& Martins, 2011; Pudjianto \& Hangjung, 2009; Raouf, Naser \& Jassim, 2012).

\subsubsection{Technology}

Tornatzky and Fleischer (1990) suggested that technology adoption is influenced by what technology is available and how the technology fits with the current internal technology infrastructure. Lippert and Govindarajulu (2006, p.148) defined the technological context as "... the available technologies important to the firm, both internal and external, that might be useful in improving organizational productivity". The technological context relates to the technologies available to an organisation and how technology characteristics themselves can influence the adoption process (Arpacı et al., 2012).

The technology context includes the internal and external technologies that are relevant to the organisation. The technology involves good ICT like fast Internet connections, sufficient up-to-date computers, and good computer networks (Raouf et al., 2012). In addition, technologies may include both equipment as well as processes.

Therefore, the technology context describes the existing technologies that are currently used and new technologies, which will be used in organisations. The technology is an important element when ICT is to be integrated to higher education.

\subsubsection{Organisation}

According to Tornatzky and Fleischer (1990) the organisation context refers to a set of features of an organisation that describes the structures and processes that either limit or facilitate the adoption of a technological innovation. Lippert and Govindarajulu (2006, p.148) defined the organisational context as "... resources available to support the acceptance of the innovation". The organisation context is vital for any IT innovation adoption in this research context. Pudjianto and Hangjung (2009) explained that researchers have utilised the TOE framework for explaining the organisational context by using various organisational factors. 


\subsubsection{Environment}

The environment context is described as the platform where the organisation operates, the industry, suppliers, customers, and government (Tornatzky and Fleischer, 1990), where all these factors influence innovation adoption. The environment context describes the environment conditions in which the organisation conducts the services (Arpacı et al., 2012). Lippert and Govindarajulu (2006, p.148) defined the environmental context as the "... setting in which the firm conducts business, and [is] influenced by the industry itself, its competitors, the firm's ability to access resources supplied by others, and interactions with the government".

Therefore, the environment includes the size and structure of the organisation competitors, macroeconomic context and regulatory environment (Tornatzky \& Fleischer, 1990).

\subsection{ICT-higher education in the Technology Context}

\subsubsection{Digital Divide}

There are many definitions of digital divide. A clear cut definition provided by Cullen (2001) was: "the gap that exists in most countries between those with ready access to the tools of information and communication technologies, and the knowledge that they provide access to, and those without such access or skills" (p.311). Doh and Stough (2010, p.55) stated that the “... digital divide means the multidimensional inequalities in ICT access, usage capacity, and usage level at the individual, community, state, and global level". In the same vein Sidorenko and Findlay (2001, p.18) gave a more precise definition where “... information haves and have-nots represents the "digital divide" (see also Gulati, 2008; Pee \& Kankanhalli, 2010; Shareef et al., 2010).

The digital divide can widen the gap between individuals advantaged by the Internet and those relatively disadvantaged by the lack of the Internet (Dada 2006; ITU, 2004; Reddi \& Sinha, 2005; Willis \& Tranter, 2006). Taking a similar view, ITU (2007) stated that the causes of the digital divide are unequal access to technology and uneven ability and knowledge to implement and use that technology (see also 
ITU, 2004; Lane, 2009; Reddi \& Sinha, 2005). The digital divide also can be characterised in relation to different types of social disparity (Lane, 2009; Shareef et al., 2010). Developing countries are often poor in e-readiness (Dada, 2006).

Despite the Internet having being available for many years, Rogers (2001) supported the view that connectivity remains the reason for the digital divide and lack of computer access to the Internet. In addition to this form of divide, Rogers claimed that other forms of divide are "educational divide", "socio-economicdivide" and "learning divide". Frédéric Riehl (Chairman of the Chairman of International Telecommunication Union Council 2007) stated that “... one of the ITU's top priorities is to assist in bridging the digital divide and reduce inequality in access to ICTs that will shape the future of tomorrow's knowledge economy" (ITU, 2007, p.6). The International Telecommunication Union was working hard to assist member countries to bridge national and international digital divides in ICT through improved infrastructure for universal access (ITU, 2007).

The Internet has been the most rapidly diffusing innovation in human history, especially since 1989, and in particular in the Asian countries in an uneven fashion (Goldfarb, 2006; Rogers, 2001). Rogers (2001) agreed that the uneven spread of the Internet has contributed to the popularity of the "digital divide" (Rogers, 2001). Rogers' (1995) and (2003) diffusion theory shows that new technologies spread unevenly (see Willis \& Tranter, 2006). Rogers' (2001) research showed that, both in the USA and on a global scale, the digital divide is a major social problem in terms of Internet diffusion.

Wims and Lawler (2007) insisted that "ICT curriculum" is a key step in bridging the digital divide. ICT usage on the outer islands in the Maldives is significantly lower in comparison to the islands of the capital (Athif \& Pimenidis, 2009). Integrating ICT into the national curriculum should be a priority if SIDS are to gain from the benefits of technology (UNESCO, 2011).

In summary, the digital divide is an inequality between groups or people in terms of access to use and knowledge of ICT. Doh and Stough (2010) argued that the 
digital divide may be different depending on the stage of development of an information society, and can exist within countries, with a global digital divide between countries.

\subsubsection{Infrastructure and resources}

A proper ICT infrastructure is crucial for ICT-enabled higher education; however developing countries often lack these infrastructures and resources (Dada, 2006). This lack of ICT infrastructure in developing countries was further highlighted by Cullen (2001) who emphasised ICT infrastructure as a mandatory prerequisite. Lack of infrastructure and the high cost of telecommunication facilities create a physical barrier to the use of technology in the educational arena (Sidorenko \& Findlay, 2001). Speaking in the context of ICT access, Kozma, McGhee, Quellmalz and Zalles (2004) pointed out that lack of access to technology reduces the prospects for developing countries to participate in the growing global economy.

Cullen (2001) added that the main barriers in ICT adaptation are physical access to telecommunications infrastructure and lack of ICT skills (see Kozma et al., 2004). Mac-Ikemenjima (2005) described inadequate ICT infrastructure, such as computer hardware and bandwidth, and ICT skilled manpower as the two major challenges faced in technology-based education. An ICT infrastructure is vitally important for the adoption of ICT-enabled higher education (UNCTAD, 2004a).

\subsection{ICT-higher education in the Organisation Context}

\subsubsection{Human Resources}

Human Development and utilisation of the human resource potential through wider employment opportunities are paramount elements for any national development plan (Country Report, 2009). Adam and Urquhart (2007) defined IT capacity building as “... the process of creating or enhancing local human and organisational abilities to use IT to perform specific tasks in organisations in order to attain organisational objectives, [...] is based on the idea of human capital" (p.317). 
SIDS needs to be internationally competitive in order to tackle the vulnerabilities of human resource development (Binger et al., 2002). The educational level in some developing countries is comparatively low and the end result produces limited human capital (Sidorenko \& Findlay, 2001). SIDS education systems are challenged in preparing high quality resources by major considerations, relating to geography, economic, social and cultural features (Bacchus, 2008).

Skilled staff are a major asset in any educational organisation and there is a strong need for them to be well trained and have skills appropriate to learning and teaching. The slow implementation of the Maldives' e-Government project and unsuccessful adoption and adaptation of information technology were due to a lack of information technology skills and human capital (Adam \& Urquhart, 2007; Aduwa-Ogiegbaen \& Iyamu, 2005). Several research studies indicated that a major problem in SIDS is a lack of trained human resource and skills due to limited resources (ITU, 2007; Kozma et al., 2004; Sidorenko \& Findlay 2001; UNCTAD, 2004a). Human capital and education are important for a country and are drivers of economic growth and social development (Sidorenko \& Findlay 2001; UNCTAD, 2004a).

As Kozma et al.'s (2004) study indicated, ICT access is a major contribution to human development. The International Telecommunication Union is playing an active role to mobilise the technical, financial and human resources needed to make the global information society a reality (ITU, 2007). According to the UNDP (2000) Human Development Report, the education system in the Maldives is not yet producing enough skilled graduates to meet internal demands.

The small population size in SIDS limits their demand for educated and trained people and results in a lack of career opportunities (Bacchus, 2008). Overseas training is very costly and, due to its limited resources, the Maldives may not be able to bear the cost of foreign training for all who wish to undertake it (UNDP, 2000). This point is true for most developing countries where the resources available for training within the country are limited (Adam \& Urquhart, 2007). IT 
training is obviously a critical factor (Adam \& Urquhart, 2007) that would help eliminate the global technology gap.

Kozma et al. (2004) explained that the use of ICT can make a difference in human development, provided there are those who have ICT access and the development of a highly skilled workforce. Adam and Urquhart (2007) stressed proper measures are necessary so that the technologies are appropriately adopted and adapted to the cultural context of the Maldives. There is also a need to promote computer literacy training and awareness among the public, particularly for those outside the workforce and academic environment and this development could be modelled on the Maldives' successful adult literacy programme (Minges \& Gray, 2004).

One of the main problems for developing countries is that the quality of the curriculum taught can be either out of date or inappropriate (Adam \& Urquhart, 2007). Lack of an Information Technology curriculum within schools and institutes constitutes a major problem in harnessing the ICT basic skills required by the students. Adam and Urquhart (2007) emphasised that the Maldives faces major problems with its lack of IT skills and stated that "lack of IT skills and human capital are major factors that contribute to the unsuccessful adoption and implementation of IT in organisations in developing countries" (p.331).

\subsubsection{ICT Policy}

Sidorenko and Findlay (2001) highlighted the importance of policies at national level for the application of any new technology. With regard to ICT policy formulation Farrell and Wachholz (2003) stated “...countries are at different stages of both development and implementation in the areas of policy formulation, ICT infrastructure development and access to it, content development, programme initiatives and the training provided for education personnel" (p.265).

Government involvement in formulating a national policy is important. Adam and Urquhart (2007) highlighted that there are two important points to consider: (a) to formulate and implement sound national context-driven information technology 
policy and (b) to increase national IT awareness with opportunities for IT skills use.

Like many developing countries, the Maldives has IT-related problems due to the lack of a national IT Policy and Strategy (Adam \& Urquhart, 2007). The Government Network of the Maldives (GNM) Master Plan gave priority to issues such as formulating a national ICT policy (MCST, 2005a; MCST, 2005b). The need for sound policies at a national level is crucial for successful implementation of ICT-based higher education models.

\subsubsection{Organisational Support and Systemic issues}

The organisational support and systematic issues are important elements for ICT integration into higher education. The legislative frameworks and organisational structures of institutes may make it impractical for ICT tools to be explored and appropriated pedagogically (Somekh, 2008). Legislative frameworks and organisational structures may “... severely constrain teachers' and students' agency, because they are in effect cultural tools that mediate pedagogies ..." (Somekh, 2008, p.450). Teachers are unlikely to use ICT in teaching and learning without intensive and active support from institutes. The structure of schools is an important factor affecting ICT integration, in the context of a systemic approach, in which policies and infrastructure should be considered (Salomon, 1991).

\subsubsection{Lack of confidence and competency of teachers}

Information and Communications Technology used by teachers can create a myriad of possibilities for instruction, making it easier for teachers and learners to use all learning styles (Flecknoe, 2002; Plumm, 2008). According to Plumm (2008), “... the number of teachers using technology in their classrooms is continually increasing" (p.1056). In the 1980s, technology in the classroom emerged as a challenge for teachers (Plumm, 2008). However, Plumm (2008) argued that teachers need to have a positive perception about using technology.

There are enormous concerns about the use of ICT tools, due to lack of competency among teachers (Bingimlas, 2009; Erstad, 2003; Littlejohn, 2002; Prain \& Hand, 
2003; Slay, Sieborger \& Hodgkinson-Williams, 2008). Bingimlas (2009) argued that, even when teachers have access to ICTs, they rarely use them effectively due to lack of confidence. Teachers' beliefs and practices in determining the level and type of technology use in the classrooms are important (Somekh 2008). Some teachers are less confident using ICT to promote complex teaching and learning (Sangrà \& González-Sanmamed, 2010).

Prain and Hand (2003) also noted that teachers resist change or lack sufficient training experience in using ICT technologies. According to Schofield (1997), students often know more than their teachers about the use of ICT technologies.

There are concerns that slow uptake of ICT by teachers (Hayes, 2007) and lack of competency among teachers cause resistance to using ICT tools (Bingimlas, 2009). Demiraslan and Usluel (2008) explained that lack of knowledge is one reason why teachers do not use ICT in courses. Some teachers are concerned about authority and react negatively to ICT tools because they detract from the teachers' role in the classroom (Schofield, 1997). Bingimlas (2009) stated, "lack of competence is one of the most important obstacles to teachers' use of technology in education" (p.242).

\subsection{ICT-higher education in the Environment Context}

\subsubsection{Socio-culture}

The socio-cultural aspect is an important phenomenon when it comes to adopting ICT into the higher education context. Taking into consideration the aspect of culture, a definition provided by Hofstede (1991) is “... the collective programming of the mind which distinguishes the members of one group or category of people from another" (p.5). A more precise definition was given by Olutimayin (2002): a "flexible set of rules that prescribe standards of proper and acceptable behaviour within a society" (p.2). A number of studies have shown that cultural perceptions toward different technologies are the key factors related to both the initial acceptance as well as the behaviour of use (Bates, 2001a; Myers \& Tan, 2002; Olutimayin, 2002). Olutimayin (2002) argued that little attention has been given to 
culture, although there is a strong belief that the use of ICT has positive effects in development.

Myers and Tan (2002) agreed that culture is important and a general consideration of the culture and its impact is useful when implementing ICT deployment in a global perspective. As Olutimayin (2002) believed, the issues of societal culture are an important element when adopting IT and changing to a "computer culture" (Olutimayin, 2002). Albirini (2006) stated, “... intellectuals from different developing nations have been aware of the difficulty involved in accommodating "the computer culture" within their home cultures" (p.51). Therefore, it is important to consider the culture aspect when ICT is to be integrated into higher education.

The social aspect is equally important aspect when adopting ICT into the higher education sector. Hsu (2007) argued that the concept of Instant Messaging (IM) could also be utilised in educational settings, due to its capacity for interactive realtime communications with immediate responses. In addition, Hsu (2007) stated that "IM not only allows students to collaborate more effectively on homework assignments and projects but also helps to maintain closer social network between students, which could have a positive impact on learning" (p.73). Social networking sites have pedagogical potential and can be used in both the academic and business worlds (Visagie \& Villiers, 2010). Somekh (2008) also indicated that ICT integration in teaching and learning is influenced by the cultural, social and organisational contexts in which students and teachers live and work.

\subsubsection{Pedagogy}

Webb and Cox (2004) provided an extensive review of research into the broad definition of "the science of teaching" (p.236). Successful integration of ICT is becoming an essential competency for educators and learners (Wang, 2008). Selwyn (2007) argued the potential of computer technologies to revolutionise tertiary institutes' teaching and learning has already been celebrated by education technologists. A research study by Somekh (2008) showed that teachers must be aware of the technology in order to adopt new roles and scaffold students' learning. 
Information and Communication Technology plays the role of a catalyst for pedagogical change (Czerniewicz, Ravjee \& Mlitwa, 2007). Wang (2008) defined pedagogy as "the teaching strategies, techniques or approaches that teachers use to deliver instruction or facilitate learning" (p.412). Wang (2008) explained that pedagogy, social interaction and technology are three components of a technologyflavoured learning environment, and a coherent design is necessary to allow teachers to integrate ICT into teaching. These studies indicated that pedagogical issues are vital ingredients in ICT integration into higher education.

The use of ICT has the potential to change current pedagogical practices in the Maldives' higher education sector, which relies on the traditional lecture approach. It is collectively accepted that ICT allows more teamwork and less didactic instruction (Aduwa-Ogiegbaen \& Iyamu, 2005).

Punie et al. (2006) explained that even most European universities are still at a stage where the use of ICT "consists of treating the computer as a sophisticated typewriter and as a means of facilitating communication via traditional pedagogy and didactics in the actual teaching situation" (p.11).

\subsubsection{Attitude towards technology}

Higher education institutes have invested significant funds to purchase new technologies (Gulbahar, 2007). However, successful use of ICT in the classroom setting depends on students' attitudes (Khan et al.. 2012). Learners within the learning environment take a keen interest in using new ICT technologies, which allows their learning to be more effective (Flecknoe, 2002). A study undertaken by Coffin and MacIntyre (1999) revealed that as "... students gain more experience with computers, their attitudes towards computers should become more positive" (p.555). As noted by Tinio (2000), Internet connectivity can increase learner motivation because it creates interactivity with other ICTs which facilitates connecting with people and collaborating in real world events.

ICTs such as videos and interactive multimedia can be used to engage the student in the learning process (Tinio, 2000). Generally, students use ICT for many 
different purposes: writing, e-mailing, Internet browsing, playing games and chatting (Erstad, 2003). When ICT is used, students are more motivated to learn (Demiraslan \& Usluel, 2008; Erstad, 2003; Ilomaki \& Rantanen, 2007) and develop skills and increase their ability to learn (Flecknoe, 2002). Plumm (2008) argued that students tend to have positive perceptions about using technology in the classroom.

Solvberg's (2003) longitudinal study found that students' inherent motivation when using ICT was high with no evidence of the novelty factor effect (see Coffin \& MacIntyre, 1999). McKinnon et al. (2000), in their study, found that an experimental group of students became enthusiastic when using computers and performed remarkably better than a non-experimental group of students. ICT does promote academic achievement, but the impact depends on how ICT is used (Saunders \& Klemming, 2003), and pleasurable and enjoyable learning activities (Podmore, 1991).

A study carried out by Demiraslan and Usluel (2008) confirmed that the use of ICT motivates students. This point was strongly emphasised by Kington et al.'s (2002) study which stated: “... the use of ICT by students for the production of their work motivated them to complete work and to redraft work to reach the required standard" (p.33). Podmore (1991) had a similar view and pointed out that "... computers are generally motivating and have holding power for children" (p.87). Schofield (1997) strongly argued that the huge body of research and experiments suggests that the use of ICT does motivate students and increases mental challenge, control and curiosity. There are issues in terms of attitudes towards technology as rightly pointed out by Kington et al. (2002), in that some students do not use computers.

\subsection{Technology Acceptance Model (TAM)}

The Technology Acceptance Model (TAM) was developed by Davis (1989). Technology Acceptance Model (Davis 1989; Davis et al., 1989), shown in Figure 3, is the most widely used model of user acceptance and usage. Researchers have used TAM to study the adoption of various technologies and TAM has become the 
most influential theory in the Information Systems field (Benbasat \& Barki, 2007; Chen, Li \& Li, 2010; Godoe \& Johansen, 2012). The Technology Acceptance Model explains that Perceived Usefulness (PU) and Perceived Ease of Use (PEOU) are beliefs about a new technology that influence an individual's attitude towards and use of that technology (Davis et al., 1989; Godoe \& Johansen, 2012).

Adoption of an IT artefact depends on two main constructs: PU and PEOU (Chen et al., 2010; Davis, 1989; Davis, 1993; Iqbal \& Qureshi, 2012; Venkatesh \& Bala, 2008). PU and PEOU determine the intention to use the system, which in turn has an effect on the actual system use. Perceived Usefulness and Perceived Ease of Use are assumed to be related to the acceptance of a computer or technology system (Chang, Yan \& Tseng, 2012; Godoe \& Johansen, 2012).

Davis (1993) explained that PEOU has a causal effect on PU since it has an indirect effect on attitudes towards and actual usage behaviour. Technology Acceptance Model suggests that perceived usefulness will be influenced by perceived ease of use because the easier a technology is to use, the more useful it will be (Venkatesh, 2000).

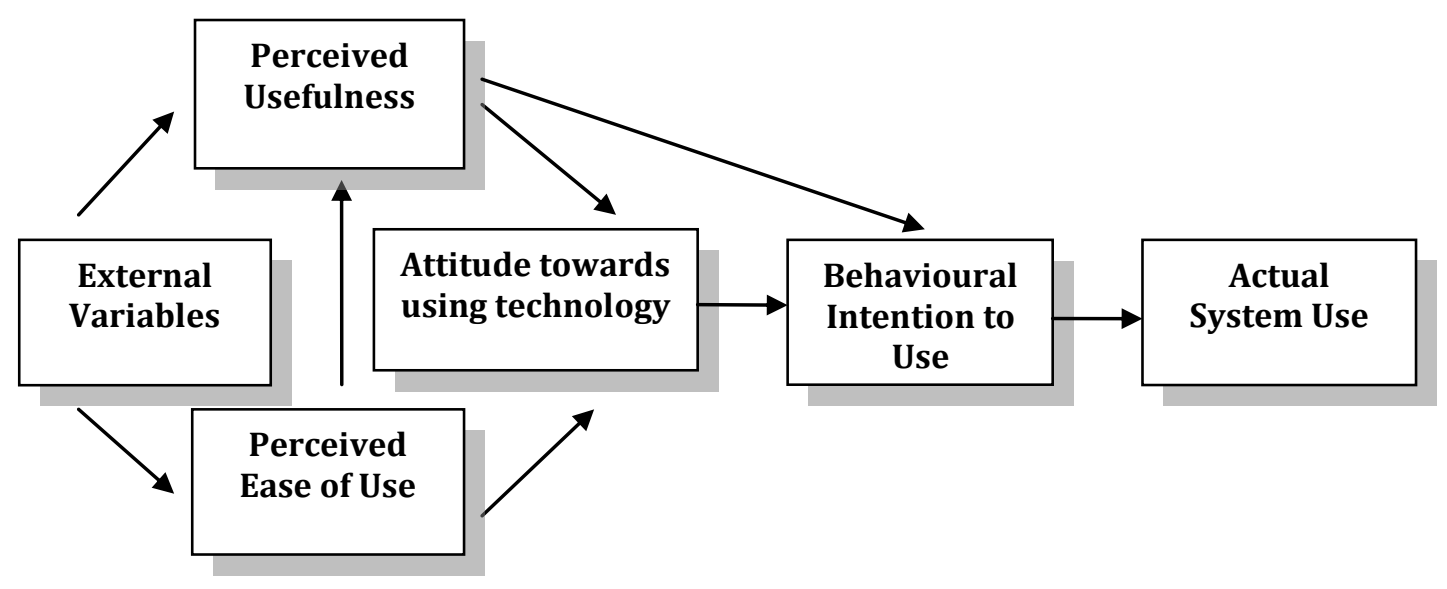

Figure 3: $\quad$ Original Technology Acceptance Model (Davis et al. 1989, p.985)

The Technology Acceptance Model is designed to predict information technology acceptance and usage on the job (Ventakesh, Morris, Davis \& Davis, 2003). The goal of TAM as explained by Davis, Bagozzi and Warshaw $(1989$, p.985) is: “ ... to provide an explanation of the determinants of computer acceptance that is general, 
capable of explaining user behaviour across a broad range of end-user computing technologies and user populations, while at the same time being both parsimonious and theoretically justified".

The Technology Acceptance Model has predictive power to enable it to apply to different situations but the model also has key limitations (Venkatesh, 2000). According to Benbasat and Barki (2007, p.212), "TAM is the most influential theory, but its the intense focus has led to a number of dysfunctional outcomes: a) the diversion of researchers' attention away from important phenomena; b) TAM-based research has led to the creation of an illusion of progress in knowledge accumulation; and c) The inability of TAM as a theory to provide a systematic means of expanding and adapting its core model has limited its usefulness in the constantly evolving IT adoption context".

\subsubsection{Perceived Ease of Use (PEOU)}

Davis (1989, p.320) defined PEOU as "the degree to which a person believes that using a particular system would be free of effort" (see also Chen et al., 2010, p.125). PEOU is a construct tied to an individual's assessment of the effort required in using the system (see Davis, 1989). Chang et al. (2012) described PEOU as the belief that a user does not expect to put much effort into making use of a particular system. If technology is skill demanding, individuals are more willing to use userfriendly systems that can achieve the same performance (Pee \& Kankanhalli, 2010).

\subsubsection{Perceived Usefulness (PU)}

Perceived usefulness is defined here as "the degree to which a person believes that using a particular system would enhance his or her job performance" (Davis, 1989, p.320). Perceived Usefulness is the belief that a user anticipates work efficiency can be enhanced by a particular application system (Chang et al., 2012). Outcome expectations are a vital antecedent to technology use behaviour, since individuals expect to solve problems in a favourable manner (Pee \& Kankanhalli, 2010). 
Doh and Stough (2010) argued that there is a belief that personal behaviour and personality are related depending on the perceived usefulness of that issue in our society. The relative advantages of IT cannot be achieved due to a lack of ICT adoption and usage is unimportant, despite the potential benefits (Doh and Stough, 2010).

\subsubsection{Attitude towards using technology}

Perceived Ease of Use and Perceived Usefulness positively affect the attitudes toward an information system and affect individuals' intentions to use and acceptance (Chen et al. 2010). Research undertaken by Chang, Yan and Tseng regarding mobile technology showed that perceived usefulness and attitude toward using had a considerable positive effect on continuance of intention to use (Chang et al., 2012).

\subsubsection{Behavioural intention of use}

Behavioural Intention (BI) was defined as the extent to which an individual intends to perform a specific behaviour (Davis et al., 1989). Both PEOU and PU positively affect attitudes toward an information system and individuals' intentions to use and the acceptance (Chen et al., 2010).

\subsubsection{Justification of TAM}

Researchers have used TAM to study the adoption of various ICT technologies. The Technology Acceptance Model has become the most influential theory in the Information Systems field (Benbasat \& Barki, 2007; Chen, Li \& Li, 2010; Godoe \& Johansen, 2012). The Technology Acceptance Model suggests that, when users are exposed to new technology, there will be several factors (i.e. PU and PEOU) that influence decisions about how and when they will use it.

As Venkatesh and Bala (2008) also noted, TAM was developed to predict individual adoption and use of new information technologies. The Technology Acceptance Model suggests that PU and PEOU are beliefs about a new technology that influence an individual's attitude toward and use of that technology (Davis et al., 1989). The researcher used TAM for this specific research since it is an Information Systems theory that models how users come to accept and use a technology. 


\subsection{Diffusion of Innovation (DOI)}

The sociologist Everett Rogers (1995) is best known for his "diffusion of innovation" theory and for introducing the term "early adopter". Rogers (2003) defined diffusion as "the process by which an innovation is communicated through certain channels over time among the members of a social system" (p.5). Diffusion of Innovation is important because it is hard to develop useful knowledge (Rogers, 2003). Diffusion of Innovation is an important theory in information system, which explains how and why technology spread through cultures (Rogers, 2003). According to Rogers, DOI is largely a social process, which means that innovations are "gradually worked out through a process of social construction" (Rogers, 2003, p. $\mathrm{xxi}$.

The word innovation describes as “... an idea, practice or object perceived as new by an individual or other unit of adoption" (Rogers, 2003, p.12; Sahin, 2006, p.14). Without diffusion, an innovation has no economic impact (Arpacı et al., 2012). The four components of diffusion of innovation are: a) an innovation; b) is communicated through certain channels; c) over time; and d) among the members of a social system (Knowlton, 2008; Rogers 1995; Rogers, 2003).

\subsubsection{Innovation}

Rogers described an innovation as an idea, practice, or object that is perceived as new by an individual or others (Rogers, 1995; Rogers, 2003). The innovation does not have to be objectively new but merely perceived as new to the people involved (Van de Ven, 1986). This innovation could be both technical (i.e. new technologies) and administrative (i.e. new procedures and policies) (Van de Ven, 1986).

Van de Ven (1986) defined innovation as "the development and implementation of new ideas by people who over time engage in transactions with others within an institutional context" (p.604). Innovation is viewed a good thing because the new idea must be useful, profitable and constructive, with any new ideas that are not useful regarded as mistakes (Van de Ven, 1986). According to Damanpour (1992), organisational innovation pertains to all parts of the organisation, all aspects of operation and all types of innovation. 
The five characteristics that determine the rate of adoption of innovation are relative advantage, compatibility, complexity, trialability, and observability (Bates, Manuel \& Oppenheim, 2007; Rogers \& Scott, 1997).

1. Relative advantage - "the degree to which an innovation is perceived as better than the idea it supersedes" (Rogers, 2003, p.229). It does not matter so much if an innovation has a great deal of objective advantage, Rogers and Scott (1997) explained that what is important is that the individual perceives the innovation as advantageous.

2. Compatibility - "the degree to which innovation is perceived as consistent with the existing values, past experience, and needs of potential adopters" (Rogers, 2003, p.15).

3. Complexity - "the degree to which an innovation is perceived as difficult to understand and use" (Rogers, 2003, p.15). Excessive complexity of an innovation is a vital obstacle in its adoption since it negatively correlates with the rate of adoption (Sahin, 2006).

4. Trialability - "the degree to which an innovation may be experimented on a limited basis" (Rogers, 2003, p.16). An innovation that is trialable presents less uncertainty to the individual who is considering adopting its use.

5. Observability - "the degree to which the results of an innovation are visible to others" (Rogers, 2003, p.16). The easier it is for individuals to see the results of an innovation, the more likely they are to adopt it.

\subsubsection{Communication Channels}

Communication is the process whereby participants create and share information with each other to reach shared understanding (Bates et al., 2007; Roger, 1995; Rogers, 2003). Communication is the heart of diffusion of innovation theory. A 
communication channel is the means by which messages get from one individual to another (i.e. through ICT).

The concept of the diffusion process is simple, as Rogers stated: "the diffusion process is the information exchange through which one individual communicates a new idea to one or several others" (Rogers, 2005, p.18). Communication is the process by which students and teachers create and share information with one another in order to reach a mutual understanding, using ICT within higher education.

Rogers identified two categories of communication channels: mass media and interpersonal communication (Rogers, 2003, Sahin, 2006). The role of mass media and Internet are considered the best channels to create awareness among users (Roman, 2003). Mass media include mass medium such as TV, radio or internet. On the other hand, "diffusion is a very social process that involves interpersonal communication relationships" (Rogers, 2003, p.19). Bates at al. (2007) argued that a combination of mass media (i.e. formal method) and interpersonal methods (i.e. informal methods) is important.

This element of the diffusion theory is important as one of the main ideas in the use of ICT is to increase the communication among teachers, learners, and the management of higher education authorities. Therefore, mass media channels are effective in creating knowledge about innovations and interpersonal channels are effective in changing attitudes towards a new idea.

\subsubsection{Time}

The time element has three phases: a) innovation-decision process; b) innovativeness; and c) innovation rate (Rogers, 1995; Rogers, 2003). Regarding the first phase, the innovation-decision process includes the five mental stages or steps that the individual passes through (Rogers, 2003) which are knowledge, persuasion, decision, implementation and confirmation (Rogers, 2003). In the second phase, the degree to which a member or members of a social system adopt 
an innovation earlier than others within the same social system is defined as innovativeness.

\subsubsection{Adopter Categories}

Rogers (1995) identified five adopter categories that reflect relative innovativeness: innovators, early adopters; early majority; late majority and laggards (see Rogers, 2003). It is believed that at the initial stage a few individuals adopt an innovation. Later the diffusion curves climbs up as the innovators, early adopters, and early majority adopt the innovation. The highest peak is somewhere between the early majority and late majority, and finally it slopes down to the laggards. The resistance to adoption is derived from research by Rogers and represented in Figure 4.

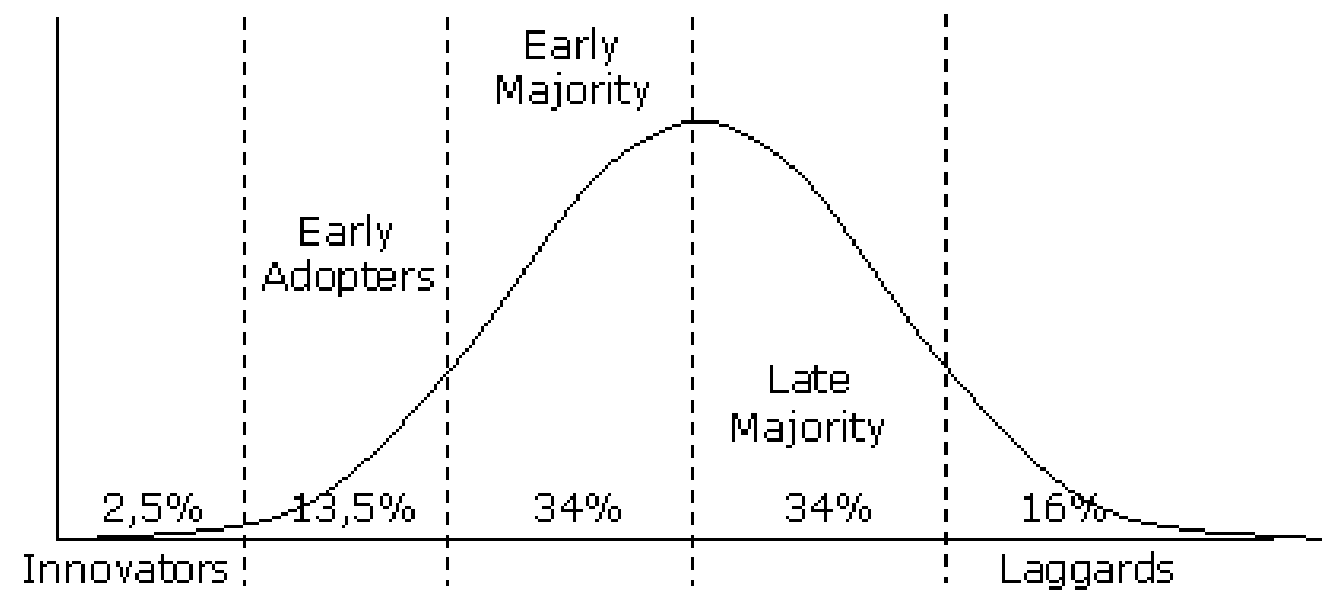

Figure 4: Rogers' Innovation adoption Curve (adapted from: Rogers, 2003, p.281)

Innovators (2.5\%): a small group of early adopters; they are enthusiasts who are committed to ICT use in higher education (Bates, 2001c). This group would be committed to change and do whatever they can to make the innovation work and are willing to experience new ideas (Rogers, 1995; Rogers, 2003). Innovators should be prepared to handle with unsuccessful innovations with a certain level of uncertainty about the innovation (Sahin, 2006).

Early Adopters (13.5\%): most important group for targeting innovative practice (Bates, 2001c). This group of people will have not yet adopted a new practice, but are open to change (Rogers, 1995; Rogers, 2003). Rogers (2003) argued this group is more likely to hold leadership roles in the social system. 
Early Majority (34\%): This group tend to be slower in the adoption process but have good interaction with other members of the social system (Rogers, 2003).

Late Majority (34\%): members are strongly hostile to change. They may have deep ideological or philosophical objections to change, or may see their status or position challenged by the change (Bates, 2001c). They are unlikely ever to embrace the innovation with a high degree of scepticism (Rogers, 1995; Rogers, 2003). The late majority includes almost one-third of all members who wait until most of their peers adopt the innovation (Sahin, 2006).

Laggards (16\%): last to adopt an innovation with no opinion leadership and are focused on traditions with caring for old ways (Rogers, 1995; Rogers, 2003). Laggards wait until an innovation is successful due to lack of awareness-knowledge and has no leadership role (Sahin, 2006).

\subsubsection{Social System}

Roger (2003, p.23) defined the social system as "a set of interrelated units that are engaged in joint problem-solving to accomplish a common goal”. An innovation "diffuses" within the boundary of a social system (Rogers \& Scott, 1997). Rogers $(1995 ; 2003)$ noted that the structure of a social system can facilitate or hinder the diffusion process. The social system is a norm which influences "the establishment of behaviour patterns of the members" (Rogers, 2003, p.37). Therefore, it may take time to learn new ICT technologies due to the social system.

\subsubsection{Justification of DOI to this study}

Rogers' DOI (Rogers, 1995; Rogers, 2003) has been chosen because it provides a general explanation for the manner in which new entities and ideas ICT over time disseminate through social system higher education (Uys, Nleya \& Molelu, 2004). The researcher chooses to use the DOI theory since it is a well-known and proven concept, which is widely used in information technology research (Mustonen-Ollila \& Lyytinen, 2003). The social side is important in order to understand the process of ICT adoption in any higher education institution. The social aspect will help to 
explain the social relationships and interactions among individuals (Knowlton, 2008).

\subsubsection{Organisational Innovation}

Organisation innovation is important since decision makers play a role in making decisions based in the elements of the model in Figure 5. Researchers have made efforts to comprehend the dynamics of different organisational components that enhance innovativeness. Individuals are seen as possessing varying degrees of willingness to accept innovations (Rogers, 1995).

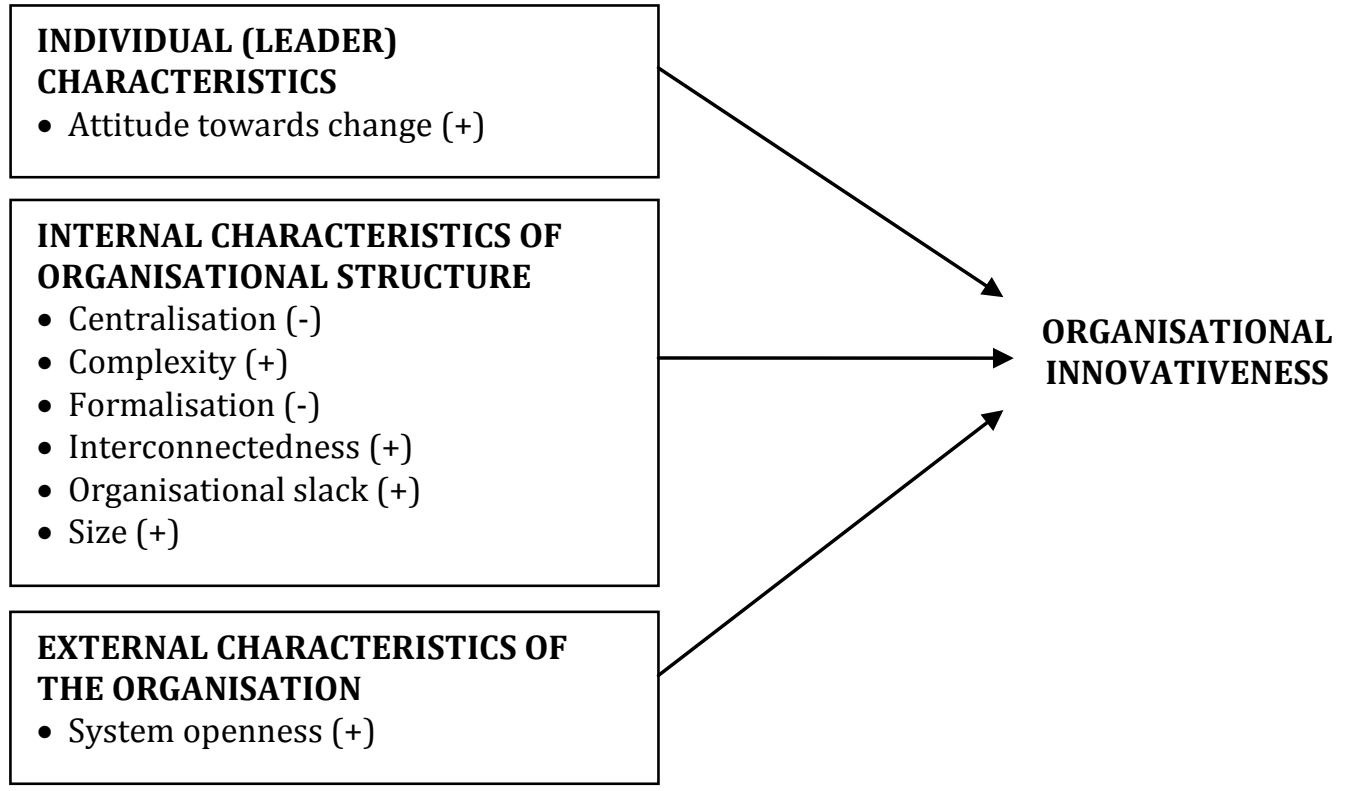

Figure 5: Diffusion of Innovation (Rogers, 2003, p.411)

Based on DOI theory at the organisational level (Rogers 1995; Rogers, 2003), innovativeness is related to such independent variables as individual (leader) characteristics, internal organisational structural characteristics, and external characteristics of the organisation (Figure 5).

a) The Individual characteristics describe the attitude towards change.

b) The Internal characteristics of organisational structure describe the Centralisation, Complexity, Formalisation, Interconnectedness, Organisational Slack and Size. 
Centralisation is the "degree to which power and control in a system are concentrated in the hands of a relatively few individuals" (Rogers, 2003, p.412).

Complexity is the "degree to which an organisation's members possess a relatively higher level knowledge and expertise" (Rogers, 2003, p.412).

Formalisation is the "degree to which an organisation emphasises its members" following rules and procedures" (Rogers, 2003, p.412).

Interconnectedness is the "degree to which the units in a social system are linked by interpersonal networks" (Rogers, 2003, p.412).

Organisational Slack is the "degree to which uncommitted resources are available to an organisation" (Rogers, 2003, p.412).

System Openness is defined "as the degree to which the members of a system are linked to other individuals who are external to the system" (Rogers, 2003, p.408).

c) The External characteristics of the organisation include System Openness only.

\subsubsection{Justification of Organisational Innovation}

Adoption refers to the decision of any individual or organisation to make use of an innovation (Rogers, 2003), whereas diffusion refers to the accumulated level of the innovation's users (Rogers, 1995). Organisational innovations can be considered as institutional changes in the utilisation of ICT within the higher education sector. The internal characteristics of the organisational structure, external characteristics of the organisation and individual leader characteristics outlined in Figure 5 are relevant to this study.

\subsection{UNESCO's continuum model of ICT development}

The use of models and frameworks is important in representing how a complex system works with a graphical representation of the major components and their relationships (Shinohara \& Nan-Zhao, 2006). 
The UNESCO continuum model of ICT development identified at least four broad stages of development in terms of adoption and use of ICT (UNESCO, 2002). The four broad approaches are: emerging, applying, infusing, and transforming (Olakulehin, 2007; UNESCO, 2002). Figure 6 shows the continuum model from the emerging to the applying to the infusing and culminates in the transforming process (Olakulehin, 2007).

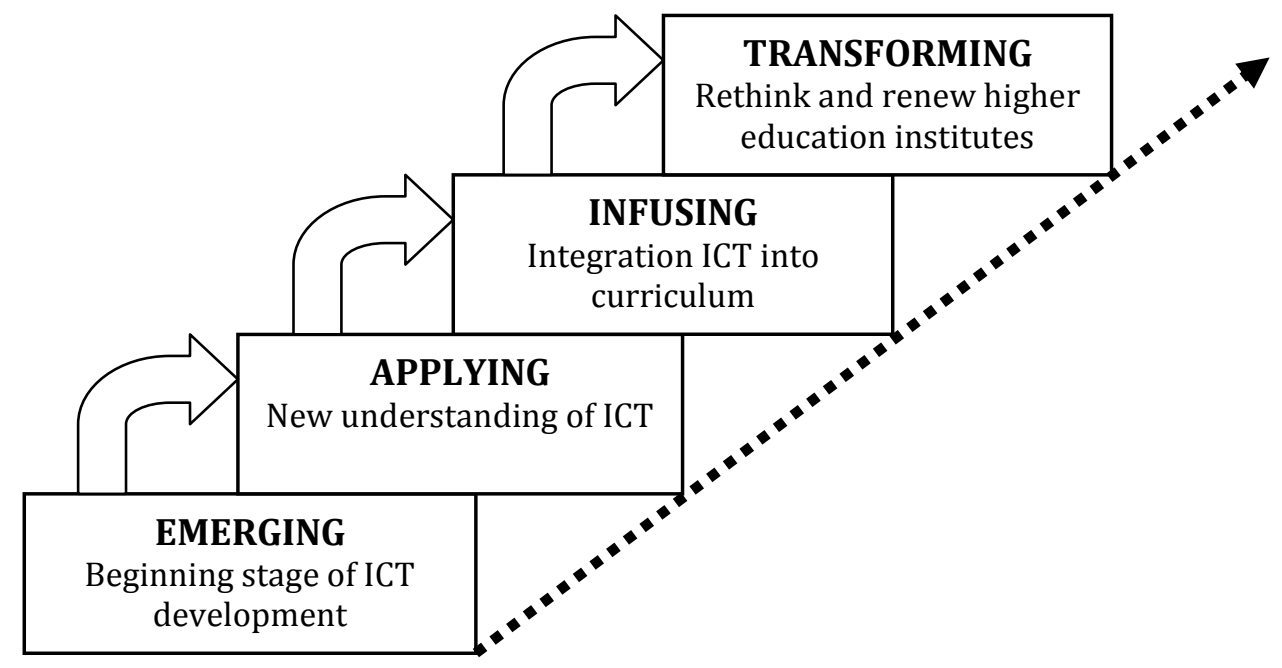

Figure 6: UNESCO's Continuum for ICT development (UNESCO, 2002, p.14)

Emerging: The emerging stage is where higher education institutes are in the initial phase of ICT development (Shinohara \& Nan-Zhao, 2006; UNESCO, 2002). In this phase, teachers and academic management will start to explore the possibilities of general use of ICT and, skills development (Olakulehin, 2007; Shinohara \& Nan-Zhao, 2006; UNESCO, 2002).

Higher education institutes at this emerging phase will still be firmly grounded in traditional methods (Shinohara \& Nan-Zhao, 2006; UNESCO, 2002) of teaching and learning. The emerging approach involves students and teachers developing their literacy skills in the use of ICT, such as word processing, use of the Internet and emailing (Shinohara \& Nan-Zhao, 2006).

Applying: This is new know-how about the contribution of ICT to learning (Shinohara \& Nan-Zhao, 2006; UNESCO, 2002). The academic management and teachers will use ICT for tasks already carried out at their institute (Shinohara \& 
Nan-Zhao, 2006; UNESC0, 2002). In the applying phase, teachers will use ICT for focusing on improving their teaching delivery, and enrich teaching and professional development (Olakulehin, 2007; Shinohara \& Nan-Zhao, 2006). The opportunity to apply ICT in all their teaching will be limited due to lack of knowhow and ICT competency and will not be fully integrated into all classroom settings (Shinohara \& Nan-Zhao, 2006).

Infusing: The infusing phase involves integrating ICT across the curriculum (Olakulehin, 2007; Shinohara \& Nan-Zhao, 2006; UNESCO, 2002). Teachers and students can explore new ways in which ICT can change professional practice (Olakulehin, 2007; UNESCO, 2002). The infusing phase of ICT infuses all aspects of teachers' and students' environments to improve teaching and learning (Shinohara \& Nan-Zhao, 2006). In this phase, teachers and students will integrate ICT into all aspects of their life in teaching and learning (Shinohara \& Nan-Zhao, 2006).

Transforming: There is a shift from the teacher-centred approach to a studentcentred approach. The focus of the curriculum will now be learner-centred, which integrates subject context with real-world applications (UNESCO, 2002). In the transforming approach, teachers and students will regard ICT as part of the everyday life (Olakulehin, 2007).

\subsubsection{Stages of teaching and learning through ICT}

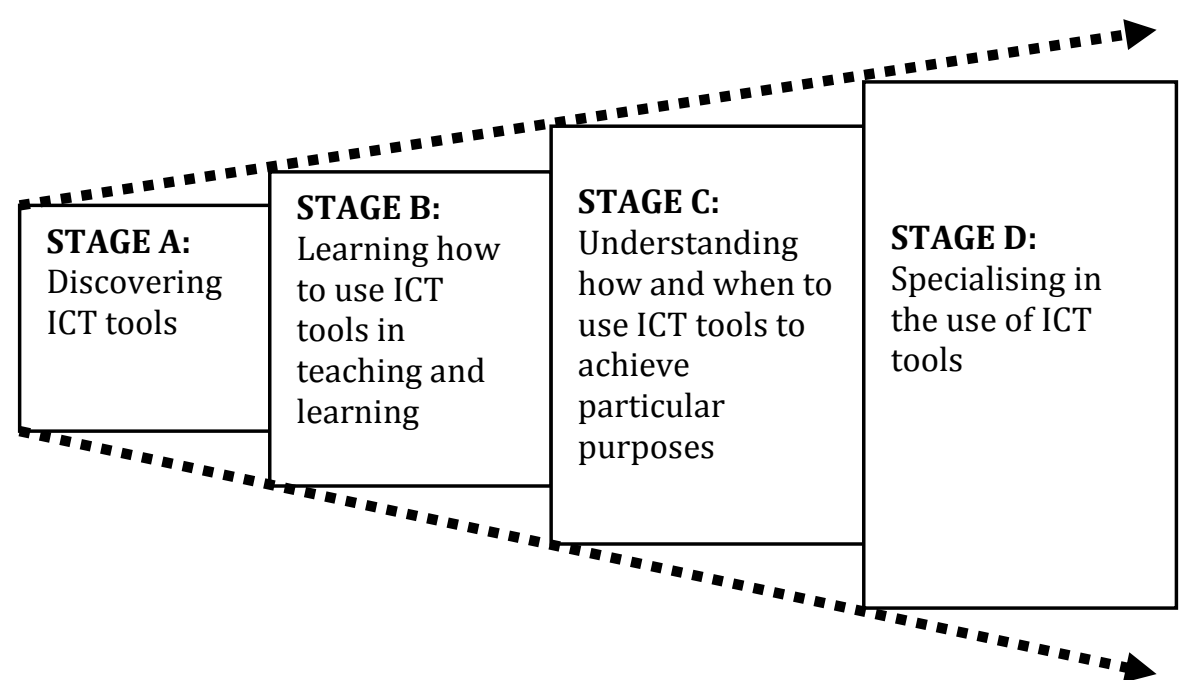

Figure 7: Models of stages of ICT development (UNESCO, 2002, p.17) 
UNESCO has formulated a four-stage continuum of learning ICT tools, which is represented in Figure 7.

Stage A: Discovering ICT tools: Students and teachers discover ICT tools in general use. This is the first stage where students and teachers will start using the ICT tools in their academic life.

Stage B: This is the stage of teaching and learning how to use ICT tools. This is the second stage where students and teachers will explore how to use the ICT tools in their teaching and learning process.

Stage C: This is the stage of understanding how and when to use ICT tools to achieve particular purposes, and includes the ability to recognise and apply ICT to tasks to solve real problems.

Stage D: This is the last stage where students and teachers specialise in the use of ICT tools and eventually become ICT specialists.

\subsection{Conceptual Model of this research}

Figure 8 provides a conceptual model of this research study's use of information system theories and frameworks. The use of a conceptual model helps to clearly show the entities, defined by Punch (2005) as “... representation, either graphically or in narrative form, of the main concept or variables, and their presumed relationship with each other" (p.53).

The researcher used one information system framework (i.e. TOE framework) and two information systems theories (TAM and DOI). The conceptual model is primarily based on the literature reviewed in this study. The literature helped the researcher to frame the problem, synthesis the knowledge base, and identify a need for this study. An integrated conceptual model of this kind helps to illustrate the components involved in this research. 
The TOE framework was used to embed the factors discovered regarding the adoption of ICT within higher education of SIDS such as the Maldives. These revealed factors are embedded within the TOE framework, as discussed in Chapter 6.

The Technology Acceptance Model was used as an information system theory to investigate how users accept and use a technology. The Technology Acceptance Model continues to be the most popularly used and practical theoretical model in the Information Systems field.

Diffusion of Innovation was used to explore how and why new ideas and technology spread through cultures.

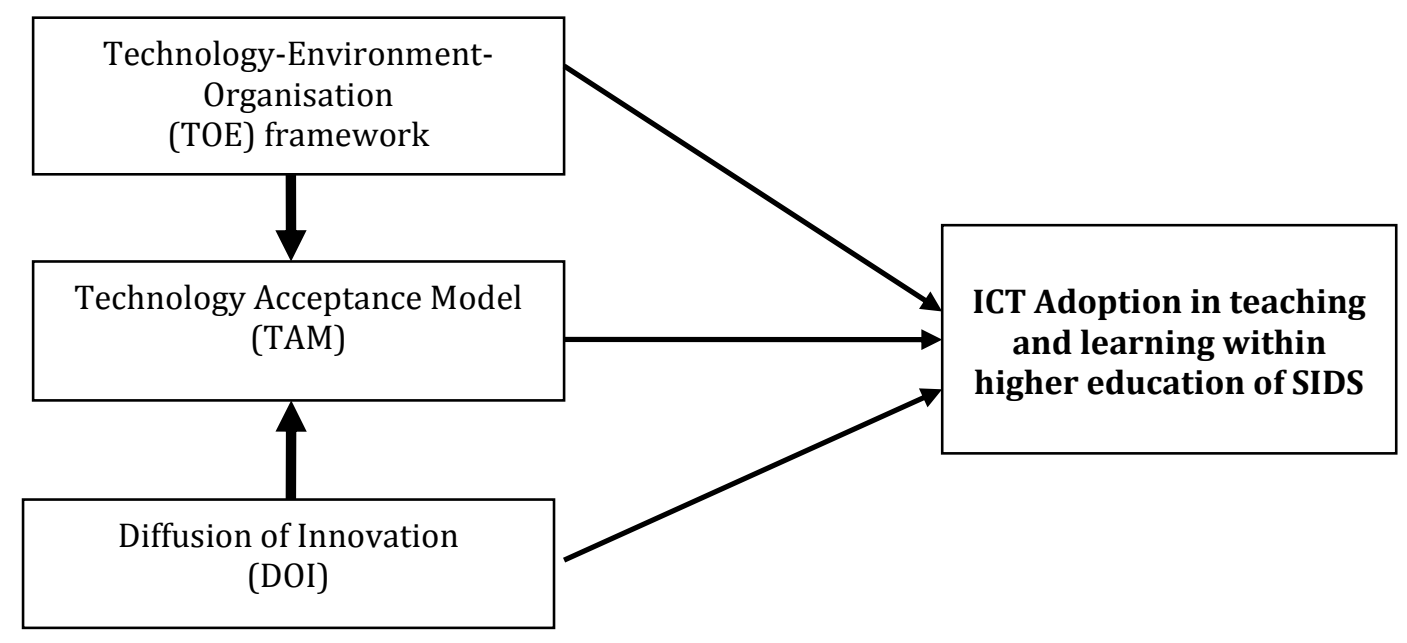

Figure 8: Conceptual Model of this research

\subsection{Gaps in Literature}

There is evidence from the reviewed literature that there is lack of ICT adoption in higher education of SIDS. There are three literature gaps noted in this research:

Firstly, there is a lack of literature identifying the factors that affect the adoption of ICT in higher education in the context of SIDS. Due to this, there is a need to undertake research about adoption of ICT in higher education in SIDS.

Secondly, there is a lack of research in the developing country and SIDS-milieu when it comes to ICT use in the higher education sector. Researchers like Walsham 
and Sahay in their research paper emphasised the importance of academic research in developing countries, by identifying gaps in the literature in this context (Walsham \& Sahay, 2006; Walsham et al., 2007).

Finally, a major gap is the need for an ICT adoption model or framework suitable for higher education in SIDS. The lack of available models and frameworks is a major problem faced by the higher education sector of SIDS such as the Maldives.

This research fills this gap in the literature and addresses the need to develop an adoption of ICT in higher education framework suitable for SIDS. This research addresses two major points: a) identification of the factors relevant to the adoption of ICT in higher education; and b) ways to utilise ICT within the higher education sector, specifically in SIDS such as the Maldives.

Research in this area seeks to address critical gaps to gain a wider understanding of future directions.

\subsection{Chapter Summary}

This chapter has reviewed the literature relevant to this research study. The chapter started by highlighting the three main elements of the research. The elements are ICT, higher education, and SIDS. This chapter also highlighted the theoretical frameworks, which are appropriate to this research. The theories and framework selected for this study are Technology-Organisation-Environment (TOE) framework, Technology Acceptance Model (TAM) and Diffusion of Innovation (DOI). The TOE framework has been selected as a useful theoretical lens to understand ICT adoption.

The main elements of TOE framework are applied in the contexts of technology, organisation and environment. Technology Acceptance Model is used in the acceptance and usage of information technology. Diffusion of Innovation is a theory that seeks to explain how new ideas and technology spread through environments. UNESCO's continuum model of ICT development is selected as a practical model for adopting ICT in the higher education context. 
The literature review in this chapter has helped to provide a general understanding about the key elements and challenges affecting adoption of ICT in higher education. The literature reviewed clearly showed there have been a limited number of studies carried out in SIDS context.

The chapter concluded by providing a conceptual model of this research study. This model uses two information system theories (i.e. TAM and DOI) and one information framework (i.e. TOE framework). A justification was provided why two information system theories and one framework was selected for this research. The literature review above lays the groundwork for this research and concludes with an identification of the research gaps this study is intended to fill. 


\section{CHAPTER 3}

\section{RESEARCH METHODOLOGY}

\subsection{Introduction}

This chapter describes the methodology used in this research. It provides a theoretical foundation for selecting a qualitative paradigm. The choices made in methodology for this research are related to the research problems, research questions and research objectives.

This chapter presents the research methodology and structured as follows:

1. Section 3.2 - provides an overview of the choice of methodology and the justifications and the epistemological stand of this research

2. Section 3.3 - describes the research design, research paradigm, and case research, explaining the sites and unit of analysis, and linking the research paradigm and methodology to the research questions and data collected.

3. Section 3.4 - explains the different data collecting methods employed in this research.

4. Section 3.5 - explains the quality of research.

5. Section 3.6 - describes the ethical considerations.

6. Section 3.7 - explains the confidentiality and anonymity considerations of this research.

7. Section 3.8 - explains the triangulation concept applied in this research.

8. Section 3.9 - summarises the chapter.

\subsection{The choice of methodology}

This part describes the development of the social science research process applied to this research and how it guides this study. In talking about research what can be known (ontology) and how it can be known (epistemology) influence the selection and choice of methodology (Hirscheim, 1992).

Ontology refers to the issues of what exists, or the basic nature of reality (Neuman, 2011). Johnson and Christensen (2008) define epistemology as “... study of knowledge, including its nature, how it is gained or generated, how it is warranted, 
and the standards that are used to judge its adequacy" (p.14). It is about the issue of how to know the world around us or make valid claims about truth (Neuman, 2011). Therefore, ontology understands knowledge and epistemology explains the study of knowledge and the accepting of valid knowledge.

A research method is the concrete techniques or procedures, which gathers and analyse the data (Crotty, 1998). In selecting the research methodology, Yin (2003) describes that there are certain characteristics to be followed such as: research topic, objectives, research questions and the nature of the research problem.

\subsection{Research Design Considerations}

A research design is the logic that links the data to be collected to the research questions (Yin, 2003). The research design in this research logically links the data and the conclusions to be drawn to the research questions of this study. Developing precise research questions and connecting them to the design, data collection and data analysis need careful planning (Punch, 2005). The nature of the research questions and the most appropriate design are important when investigating a problem (Merriam, 1988).

\subsubsection{Interpretive Paradigm}

For positivist researchers, good understanding is created by developing initial hypotheses, then testing them against the external reality (Crotty, 1998; Myers, 2006). This means positivist epistemology explores what happens in organisations through scientific measurement of the behaviour of people and systems.

Walsham (2006) explains the interpretive paradigm as “... interpretive methods of research starts from the position that our knowledge of reality, including the domain of human actions, is a social construction of human actors" (p.320). Interpretive research can help the information systems researcher to understand human thoughts and actions in social and organisational contexts (Klein \& Myers, 1999; Walsham, 2006). A simple explanation of the interpretive approach was given by Crotty (1998), as looking "for culturally derived and historically situated interpretations of the social life-world" (p.67). Interpretive research assumes "... 
our knowledge of reality is gained only through social constructions such as language, consciousness, shared meanings, documents, tools, and other artifacts" (Klein \& Myers, 1999, p. 69). This research employs an interpretivist paradigm in which knowledge can be created and understood through the view of the individuals who live and work in the context of a culture or organisation.

The importance of the interpretive paradigm to qualitative research was highlighted by Neuman (1997): "qualitative social research relies largely on the interpretive and critical approaches to social science" (p.328). The interpretive paradigm believes in the existence of multiple realities perceived by different human actors at different places. An Interpretive paradigm is relevant to this research because it helps comprehend the meaning of the real world understanding through the viewpoints of individuals (Myers, 2006). The perceptions of people are sorted out through personal experiences, and therefore we can never be objective about the interpretations made by others.

Interpretivist epistemology assumes that something can be done in a particular situation and makes sense of what will happen or is happening based on our experience and expectations (Myers, 2006). Neuman (1997) stated that the interpretivist approach is "... the systematic analysis of socially meaningful action through the direct detailed observation of people in natural settings in order to arrive at understandings and interpretations of how people create and maintain their social worlds" (p.68).

The interpretive approach ensured a sufficient dialogue between the researcher, interviewees, and focus groups respondents in order to collaboratively construct a meaningful reality. The interpretive approach is better than the positivist approach in this context because the social world can only be understood by studying the meaning of human constructs and their interactions. Based on the above discussion, the researcher believed that the philosophical stance of this research is located closest to the "interpretive paradigm". 


\subsubsection{Qualitative Approach}

Johnson and Christensen (2008) defined qualitative research as "... research relying primarily in the collection of qualitative data (non-numerical data, such as words and pictures" (p.388). Qualitative research is a situated activity that consists of a set of interpretive, material practices that make the world visible (Denzin \& Lincoln, 2000). This research required an in-depth analysis and approach, which used a qualitative approach as one of the data collecting approaches. Creswell (2009) explained qualitative research as "... exploring and understanding the meaning individuals or groups ascribe to a social or human problems" (p.4).

Punch (2005) and Pickard (2007) explained that qualitative research allows the researcher to understand people's attitudes, behaviours, value systems, concerns, motivations and the culture within which they live. Qualitative researchers explore or discover phenomena in an open-ended way and they prefer to study them as they naturally occur and view human behaviour as dynamic and changing (Johnson \& Christensen, 2008). Creswell (2009, p.176) stated ".. qualitative research is a form of interpretive inquiry in which researchers make an interpretation of what they see, hear, and understand".

In providing a detailed explanation of research participants' perspectives, the researcher has attempted to demonstrate the lived meaning of their experience through various data collection methods. This study attempted to explicitly adopt a qualitative approach, which was deemed as the most appropriate approach because by its very nature it allowed the researcher to develop an understanding of the meaning and nature of real life situations.

\subsubsection{Linking research paradigm and methodology to research questions}

The research paradigm (i.e. interpretivist) and research methodology (i.e. qualitative approach) align with the two research questions presented in this study: a) What are the factors that influence the adoption of ICT within higher education teaching and learning in SIDS such as the Maldives?; b) How can ICT be used to address the issues within the higher education teaching and learning of the SIDS such as the Maldives? 
The first research question deals with the specific factors influencing the adoption of ICT in higher education teaching and learning of SIDS such as the Maldives. This question relates to the theory of analysing (Gregor, 2006) by including a "what" question. The results of this research question produced a constructivist interpretation of the factors related to the adoption of ICT within higher education. The data were collected through qualitative data collection methods such as interviews, focus groups and a qualitative survey.

The second research questions addresses the issues discovered in the first research question and exploring how ICT can be used in higher education teaching and learning of SIDS such as the Maldives. This question relates to the theory of explaining or understanding (Gregor, 2006) because it includes how and why phenomena occur. Gregor (2006) further explains “... theory of explaining in IS that fits the interpretive paradigm..." (p. 624). The theory of explaining corresponds closely to some aspects of the interpretive paradigm (Klein \& Myers, 1999). Therefore, the second research question closely relates to the qualitative interpretivist approach, which is outlined in this research.

In qualitative and interpretive case research, the researcher is directly involved not only in the process of data collection but also in the analysis of the data gathered (Creswell, 2003). These data came from interviews, focus group discussion, a qualitative survey and document analyses - all obtained with the sole objective of addressing the research questions and, as such, qualitative interpretations (of one kind or another). The analysis followed the ultimate aim of the study to develop models, frameworks and theoretical constructs to explain the phenomena under investigation as well as bring about a better understanding of their relationships.

\subsubsection{The Research Methodology: Case Research}

There are several ways of doing social science research: one method is case research. For this research study, the definition of case research is based on the work of Bonoma (1985, p.203-204), Benbasat, Goldstein and Mead (1987, p.370); Eisenhardt (1989, p.534), and Yin (1994, p.13). However, Wilson (1987) defined 
case research studies as “... intrinsically incomplete: they are all based on the assumption that a case is defined by its boundaries in time and space" (p.43).

It is difficult to provide a straightforward definition for case research, but it can be done through documenting its strengths and weaknesses in capturing reality by studying phenomena in natural settings (Cavaye, 1996). Case research typically combines different data collection methods such as interviews, observation, questionnaires, and document analysis (Darke, Shanks \& Broadbent, 1998). Case research investigates phenomena in order to gain an in-depth understanding.

Case research can be carried out either by taking a positivist or an interpretivist stance; using qualitative and quantitative methods; and using one or multiple cases (Cavaye, 1996; Dooley, 2002; Merriam, 1988). The power of case research is the ability to use all methodologies within the data collection process and to compare within cases for research validity (Dooley, 2002).

Case research can also lead to new and creative insights and development of new theory, and multiple means of data collection increase validity (Voss, Tsikriktsis \& Frohlich, 2002). Case research is useful when a phenomenon is complex and indepth investigation is required (Benbasat et al., 1987; Bonoma 1985; Paré, 2004). Case research can be used in an interpretive manner, in order to understand the nature of a phenomenon and draw out meaning from the social setting (Cavaye, 1996). From the above literature by different authors it is clear that case research methodology has the power to use multiple data collection techniques, triangulate data and investigate multiple cases.

Case research methodology was chosen as the most appropriate approach to this study because of its ability to (a) provide a variety of participants' perspectives (b) use multiple data collection techniques and (c) examine technology integration (Voss et al., 2002).

This researcher believed that the case research approach would bring better understanding of the existing issues of the SIDS' context and would broaden the 
students', teachers', experts', consultants' and stakeholders' understanding. In addition, case research could examine the interplay of all the variables in order to provide a complete overview of the situation.

\subsubsection{Exploratory Case Research}

An exploratory investigation is used where the variables are unknown and the phenomenon not at all understood (Voss et al., 2002) because of a problem that has not been clearly defined. Most case studies are exploratory in nature (Benbasat et al., 1987).

The results of exploratory case research can be useful for decision-making and also provide insight into this research situation. Exploratory case research can reveal how the SIDS' higher education sector is coping with the situation and what the issues of concern are. This goal of this study was to find out the status quo in the sector in terms of ICT in higher education and to investigate the phenomenon without explicit expectations. Therefore, the researcher opted to use exploratory case research methodology.

\subsubsection{Definition of a "Case"}

A "Case" itself is an account of an activity, event, or problem (Dooley, 2002; Johnson \& Christensen, 2008). Stake (1995) explained that a case may be simple or complex. Johnson and Christensen (2008) defined a case as a bounded system, where "bounded" means having certain parameters such as actual event, location and time. "System" means a set of interrelated elements that form an organised whole.

Case research is the examination of a specific phenomenon such as an event or institution, where the phenomenon should be identified and the "Case" is selected as an instance of some concern (Merriam, 1988). Punch (2005, p.145) stated that there are four characteristics of a "Case":

1. The case is a "bounded system" in which the boundaries should be defined as clearly as possible; 
2. The case is a "case" of something;

3. The wholeness, unity and integrity of the case must be preserved; and

4. Multiple sources of data and multiple data collection methods are used in a naturalistic setting (Creswell, 2003; Dooley, 2002).

This research has clear boundaries because it specifically deals with higher education in SIDS. The system is a multiple interaction of things within a functioning whole. For this research, system means the higher education sector.

The single case considered in this research is "The Maldives". There are four subcases with the majority of the research participants from these higher education institutes, and the higher education and information technology sectors.

\subsubsection{Single Case}

Central to case research design is the decision whether to include one or several cases (Benbasat et al., 1987). The appropriate number of cases depends on how much is known about the phenomenon and what new information is likely to emerge from the cases (Eisenhardt, 1989). Cases can either be single or multiple (Darke, Shanks \& Broadbent, 1998; Meyer, 2001).

The number of cases to be studied depends on the research question, and rich description of that particular case. A problem of single cases is their limitation in terms of generalisability and information-processing biases (Eisenhardt, 1989). Multiple-case designs allow literal or theoretical replication and cross-case comparison. There is no ideal number of cases (Darke et al., 1998) and selecting cases is an important aspect of case methodology (Benabasat et al., 1987; Eisenhardt, 1989; Yin 2003).

The choice of the number of cases (single or multiple) depends on the time available for gathering data (Pare, 2004). It is crucial to decide on the number of cases in the case research design approach (Benbasat et al., 1987). In this research 
different groups were selected, comprising three higher education institutes, all situated in Male'. The research participants from these different groups were:

- Students - students studying in the higher education institutes;

- Teachers - teachers who are teaching in the higher education institutes;

- Stakeholders - top academic managers, decision makers, and higher education personnel;

- Consultants and Experts - information technology personnel, experts, systems system administrators, and higher education institutional managers.

The researcher was able to contact key stakeholders to obtain answers for the research questions. Choosing the right stakeholders was paramount since the Maldives is small with a limited number of people in the higher education sector. These key stakeholders could be influential in changing the existing ways of doing things within the Maldives' higher education system.

\subsubsection{Unit of analysis}

As a crucial part of the research design process, the case researcher needs to decide the unit of analysis, which involves setting the boundaries of the case and selecting the issues to emphasise (Yin, 2003). The researcher should determine the most relevant unit of analysis prior to searching for research sites (Benbasat et al., 1987). Identifying the unit of analysis defines what the "Case" is and there can be more than one unit of analysis (Yin, 2003). The unit of analysis for qualitative research should focus on words rather than numbers (Denscombe, 1999). Therefore, this research has the unit of analysis, "Adoption of ICT in higher education in SIDS such as the Maldives". 


\subsubsection{Nature of data required to answer the research questions}

This research required qualitative data to answer the two research questions raised:

1. What are the factors that influence the adoption of ICT within higher education teaching and learning in SIDS such as the Maldives?

2. How can ICT be used to address the issues within the higher education teaching and learning of the SIDS such as the Maldives?

This research required qualitative data whereby research participants speak in their own words and offer their perceptions, which provided the researcher with deep understanding by painting a rich picture of the context. The data also helped the researcher to understand the issues and how individuals or groups gave meaning to and expressed their understanding of themselves. Obtaining the correct data from reliable sources is an important part in any research discipline.

\subsection{Data collection methods}

Figure 9 represents the four data collection approaches, which were used in this research. All these data collection methods were qualitative in nature and data was analysed by using thematic coding methods.

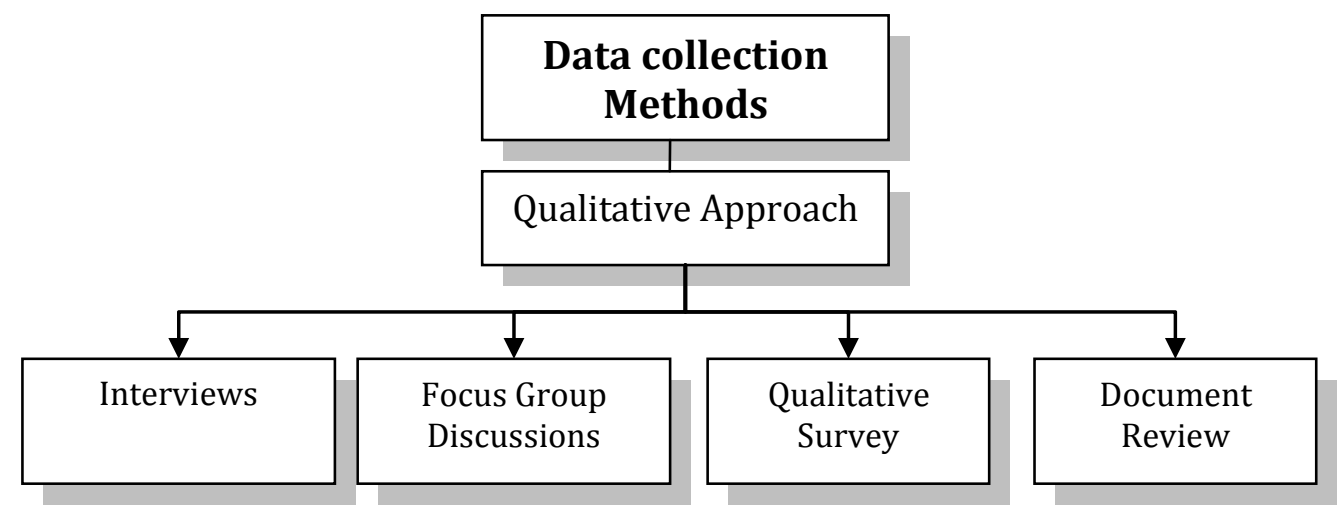

Figure 9: Data collection methods used in this research

\subsubsection{Interviews}

Qualitative data can be collected in various major forms such as interviews, which may be recorded and later transcribed (Cavaye, 1996; Dubé \& Paré, 2003). An 
interview is an interpersonal communication in which the interviewer can establish a rapport with the interviewee (Johnson \& Christensen, 2008). Like other qualitative methods, case research often has heavy reliance on personal interviews (Bonoma, 1985; Stuart et al., 2002).

Interviews are a part of most interpretive studies and a primary method of accessing the interpretations of informants in the field (Dooley, 2002; Neuman, 1997; Pickard, 2007; Walsham, 2006; Yin, 1994). Interviews are a good way of accessing people's perceptions, meanings, definitions of situations, and their construction of reality (Punch, 2005; Yin, 1994). Interviewing provides access to people's behaviour and enables researchers to comprehend the meaning of that behaviour (Seidman, 1998).

Seidman (1998) defined the interview as "... understanding the experience of the other people and the meaning they make of that experience" (p.3). An interview also has the advantage of probes - i.e. prompts used to obtain responses and additional information (Johnson \& Christensen, 2008). Neuman (2011) explained that the researcher should know when to use probes such as to clarify an ambiguous answer, incomplete answer or finding relevant responses. Therefore, the researcher chose to use interviews in this study as they are one of the data collection processes in qualitative research.

\subsubsection{Interview Format}

Research interviews comprise three different forms: structured, semi-structured and unstructured (Pickard, 2007). Semi-structured interviews give participants room to elaborate experiences and to express their own ideas and suggestions which would not be achieved by structured interviews (Nandhakumar \& Jones, 1997; Pickard, 2007, Punch 2005).

Semi-structured interviews allow the researcher to capture and explore predefined questions and therefore enable a wider range of interpretations to be found within the research area. The less the degree of structure within an 
interview, the more chance the researcher may have to explore the respondent's answers and non-standard use of terms (Nandhakumar \& Jones, 1997).

The open-ended nature of semi-structured interviews defines the research area and provides opportunities for both researcher and interviewee to discuss some topics in more detail. Based on this, the researcher undertook 32 semi-structured interviews. Seidman (1998) stated that an open-ended question "... establishes the territory to be explored while allowing the participant to take any direction he or she wants" (p.69). The interview guidelines and questions are attached (see Appendix 1). The interview guidelines have three parts: introductory components of the interviews, question format and closing components. The interview questions were based on the research problems and research objectives, in order to answer the two research questions.

\subsubsection{Selecting interview participants}

The participants included higher education managers, institute heads, teachers, and students, and experts from the education and information technology sectors. There were people from the islands outside Male'. The researcher believed it was important to include participants from rural areas. The researcher contacted the potential participants and asked for permission for an interview. Very people refused to provide an interview or participate in the focus group discussions due to lack of understanding about the topic.

\subsubsection{Recording Data}

Four main advantages of audio-recording are a) it gives a truer record of what was said compared with the taking of notes during the interview; b) it is possible to return to the transcript later for comments; c) it is useful for picking out direct quotes when writing up; and d) it frees the researcher's concentration in order to engage with the interviewee (Walsham, 1995a; Walsham, 2006).

Seidman (1998) explained the importance of recording during an interview "... if something is not clear in a transcript, the researchers can return to the source and check for accuracy" (p.97). The interviews and focus group discussions were 
audio-recorded digitally so as to preserve them for further use (Stuart et al., 2002) and enable them to be transcribed later. Audio-recording of interviews is a means of providing a complete description of the interviewees' responses (Darke et al., 1998).

After recording, the researcher translated and transcribed the interviews and focus group discussions. Transcribing audio-recorded versions is a time consuming and costly procedure (Seidman, 1998). The researcher spent about four hours of transcribing time for each hour of interview or focus group discussion.

\subsubsection{Focus Group discussions}

Focus groups are a good method for eliciting respondents' perceptions, attitudes and opinions (Pickard, 2007; Wilson, 1997). The focus group is one vital data collection method used in qualitative research. Denscombe (1999) described the focus group as “...an extremely popular form of interview technique” (p.115). The use of focus group discussions is a useful way of collecting data (Creswell, 2003) and a competent qualitative researcher may utilise the focus group method (Wilson, 1997). Focus groups allow the capturing of rich data from the participants (Morgan \& Spanish, 1984).

Both focus group discussions and interviews have four qualities: a) allow the exploration of a topic; b) talk about the things that are significant; c) serve as a useful guide to further data collection; d) explore issues more in-depth (Pickard, 2007).

The focus group guidelines and questions are attached (see Appendix 2). The focus group guidelines have three parts: introductory components of the focus group session, question format, and closing components.

\subsubsection{Structure of Focus Groups}

Different authors believed differently about the ideal size of focus groups. The ideal sizes of a focus group suggested by three different authors are: six to ten 
people (Pickard, 2007); six to eight people (Punch, 2005); and six to nine people (Denscombe, 1999).

In this research focus groups were another method of qualitative research, in which a group of people were asked questions about their perceptions and about ICT use in higher education teaching and learning. It is very important to get the correctly-balanced group composition; this research comprised four focus groups.

\subsubsection{Selecting focus group participants}

The researcher found students and teachers from the higher education institutes to contact for the focus group sessions. The first and second focus groups comprised information technology degree programme students. The third group had students from the business studies degree programme. The fourth group had teachers, consultants and experts from the information technology and education sectors. The researcher acted as an "observer" in the focus group discussions. The total duration of the each focus group discussion was about 90 minutes with two sessions for each group.

\subsubsection{Undertaking a focus group discussion}

Focus group discussions centre around five to six questions and questions are qualitative and open-ended. To make the focus group discussions effective and unbiased, the focus group sessions were conducted by a skilled moderator.

A "single moderator focus group approach" was used where the moderator ensured the session progressed smoothly and in an unbiased manner. The moderator is confident in initiating discussions and was trained by the researcher. The moderator should have the group dynamics skills and ability to ensure the group stays focused on the questions and maintain the flow of discussions (Denscombe, 1999).

A skilled moderator has three roles: formal direction, steering the topic and steering the dynamics (Flick, 2009). It is important in a focus group to manage the group dynamics to cultivate natural conversation and discussion (Punch, 2005). 
Group dynamics were apparent in all four focus group discussions of this study since all participants contributed well and expressed their candid views. Group dynamics in some focus groups created more thinking about the topic which led to in-depth discussions. The moderator's goal was to generate the maximum number of different ideas from the focus group participants in the time allocated and also ensure that the whole group was not dominated by one or two members.

\subsubsection{Qualitative Survey}

The term "survey" usually refers to a study that is used to investigate a representative sample. Punch (2005) defined a survey as "... any research which collects data from a sample of people" (p.75). The basic idea of the qualitative survey in this research was a fact-finding exercise to find out the existing ICT utilisation among students, teachers and academic managers at higher education institutes. This survey allowed the researcher to analyse the status quo of the existing situation of ICT usage within the higher education sector of the Maldives.

This qualitative survey consisted of general demographic information with 3-4 open-ended questions. The interpretive questions were similar to the type of questions asked in the interviews and focus group sessions. The surveys were distributed in two forms: paper based and electronic format (if required). The paper based survey forms were distributed to the participants who were willing to participate. A sample qualitative survey form attached (see Appendix 3).

\subsubsection{Selecting qualitative survey participants}

The participants in the qualitative survey included students and teachers. The researcher selected students from two research sites and potential participants were asked to participate. Participation was voluntary and anyone could withdraw at any time with no negative repercussions. The researcher emphasised the voluntary nature of qualitative survey participation.

\subsubsection{Undertaking Qualitative Survey}

The researcher visited the higher education institutions to meet with the students and distributed the survey forms in their respective classes. A briefing about the doctoral research and the objective of this qualitative survey was given to the 
participants. The researcher prepared an information sheet with a consent form survey participants (see Appendix 4). An assurance was given to the participants that participation in this survey was entirely voluntary; that there would be no repercussions for non-participation. The researcher scrupulously protected the anonymity of the qualitative survey.

\subsubsection{Data Analysis process}

The interview and focus group data was analysed based on the identification of themes in the qualitative open-ended questions, identified by means of a coding scheme. NViVo is a useful tool, which allows researchers to categorise chunks of the text into particular code or themes.

\subsubsection{Document Reviewing (Secondary data)}

Documents are data and can be used as important resources for research (Flick, 2009). Secondary data are existing documents originally collected at an earlier time by a different person for different purpose (Johnson \& Christensen, 2008). Secondary data may include formal reports, organisation charts, letters, memoranda, agendas, administrative documents, or any document that is relevant to the research (Benbasat et al., 1987; Denscombe, 1999; Paré, 2004). Johnson and Christensen (2008) stated “... [the] researcher must, however, find these data or artifacts so as to use them in his or her research" (p.217).

Documentation is particularly useful for tracing the history of organisations and statements made by key people in the organisations (Meyer, 2001). The document reviewing should be accurate, reliable and free from errors (Flick, 2009).

Document reviewing was important in this research since there were published and unpublished documents, which were relevant to this research. These documents were found in the education sector, higher education institutes and government organisations.

\subsection{Quality of qualitative research}

Reliability and validity are essential components for quality in quantitative research (Golafshani, 2003). In qualitative terms reliability means dependability 
or consistency and validity means truthfulness (Neuman, 2011). There are five approaches to quality within qualitative research underlined here: trustworthiness, reliability, transferability, dependability, and conformability (Cho \& Trent, 2006; Golafshani, 2003).

\subsubsection{Trustworthiness in qualitative research}

Weber (2004) explained that researchers within the interpretive paradigm propose criteria for evaluating knowledge claims like credibility, transferability, dependability and confirmability to confirm trustworthiness. Golafshani (2003) stated that “... to ensure reliability in qualitative research, examination of trustworthiness is crucial" (p.601). Qualitative researchers strive to adhere to the main principles of truthfulness (i.e. validity) (Neuman, 2011).

Creswell (2009) believed that truthfulness is one of the strengths of qualitative research since it determines whether the findings are accurate from the perspectives of both researcher and participants. Therefore, the trustworthiness of a qualitative study can be increased by maintaining high credibility and objectivity. Trustworthiness was important to this research since truthful information is necessary to build a viable thesis.

The researcher ensured that the participants provided truthful information by picking trusted research participants. In addition, the researcher also ensured trustworthiness by demonstrating that the documentation of evidence for the results was sound and the arguments were strongly justified. The researcher is confident about the truth of this study's findings based on the research design, research participants' perspectives and discovery of human experiences in the research context.

\subsubsection{Credibility}

Credibility means that the results of qualitative research are credible or believable from the perspective of the participants in the research (Trochim, 2006). Credibility in qualitative research involves close involvement with the research participants, triangulation of the techniques used to gather data, and member 
checking (Flick, 2009; Pickard, 2007). Member checking is a technique used to submit the interpretations to the scrutiny of the individuals upon whom they are based, and to seek their responses as to the interpretations' authenticity (Rowland, 2003; Seale, 1999).

The success of the research depends on credible inputs from the research participants, and it was therefore necessary for the researcher to ensure that credibility was maintained in order to make this research fruitful and consistent with the raw data.

\subsubsection{Transferability}

Transferability refers to the degree to which the results of qualitative research can be generalised to other contexts (Trochim, 2006). In qualitative research the goal is to allow for transferability of the findings rather than wholesale generalisation of those findings (Pickard, 2007). The researcher ensured that, to allow transferability, the research participants provided sufficient information about the research context to determine whether the existing environment was similar to other SIDS' situations and the findings could justifiably be applied. Transferability is important with respect to this research since it was necessary to maintain the quality and ensure generalisable findings are possible for other SIDS.

\subsubsection{Dependability}

Dependability means whether the same results appear when the same thing is done twice (Trochim, 2006). Dependability is important with respect to accuracy of transcripts (Pickard, 2007). Dependability also relates to repeating the operations of the study with similar expected results (Rowland, 2003). In other words, there can be numerous interpretations of the same data. The term "dependability" in qualitative research corresponds to the notion of "reliability" in quantitative research (Golafshani, 2003). Dependability is also proposed as a replacement for consistency, or reliability (Seale, 1999).

Dependability criteria are difficult to satisfy within qualitative work. Data on the same real life situation can be collected by different researchers, using different 
research methodologies, at different times. Neuman (2007) explained that the data sets from different settings may not collaborate into one conforming picture.

A considerable amount of time was spent in planning to ensure the interview and focus groups proceeded smoothly and captured the necessary information required to assess the research participants' perspectives. Dependability evaluates whether the process of research is logical, traceable and clearly documented. Triangulation was another point considered in this research in order to strengthen its dependability.

\subsubsection{Confirmability}

Confirmability refers to the degree to which the research outcome could be confirmed by others (Trochim, 2006). Confirmability is vital to limit the researcher's bias (Pickard, 2007). The goal of confirmability is to ensure that the results of the research can be traced back to the raw data (Pickard, 2007). To achieve confirmability, the researcher sought to demonstrate that the findings emerged from the data only and not his own predispositions.

\subsection{Ethical Considerations}

There are ethical issues in interpretive research, but very little has been written about this topic in Information Systems literature (Walsham, 2006). A researcher must ensure research participants are treated ethically (Johnson \& Christensen, 2008). Qualitative researchers may be ethically conflicted as their research may entail some degree of personal involvement in the lives of research participants. Special attention was given in this research to ensure ethical conduct in order to maximise beneficial effects and minimise harmful consequences.

To address the ethical considerations, all participants were given the participant information sheets (see Appendices 4, 8 and 10). The participants read the information sheets, which clarified doubts and answered all their queries. 
The Human Ethics Committee of The School of Information Management at Victoria University of Wellington granted ethical approval to conduct this research (see Appendix 5).

\subsection{Confidentiality and Anonymity}

Seidman (1998) believed that researchers take appropriate steps to protect the identity of the participants. Neuman (2011, p.152) defined anonymity as "... ethical protection that participants remain nameless; their identity is protected from disclosure and remains unknown". Anonymity means that recorded data is kept secretly and does not identify who it came from or refers to. Nobody knows whom it is about with certainty.

Confidentiality means "the identity of the participant will not be revealed when using any data provided by that participant" (Pickard, 2007, p.77). In other words, in this study, the researcher kept the raw data to himself and did not release it to others. The researcher only included the data in the findings that illustrated the conclusions without identifying the research participants. Neuman (2011, p.153) defined confidentiality as "the means of that we may attach names to information, but we hold it in confidence or keep it secret from the public".

In this research confidentiality was important because the integrity of the findings depended largely on the frankness of participants to disclose information about the research context. Although, it was difficult to maintain confidentiality in a small country like the Maldives, the small size is the reason why confidentiality was all the more important. Due to the small communities it would be difficult to hide the fact that people had attended a focus group or interviews. Nevertheless, the researcher ensured that what participants said was kept confidential by using pseudonyms (e.g. "survey respondents") in the documentation. The qualitative survey responses were anonymous, which offered more protection.

The researcher established an open and trusting working relationship with the participants for the entire duration of the study. Most importantly, care was taken to obtain the informed consents of participants and institutions, and to protect 
confidentiality. Therefore, confidentiality and anonymity were ensured for the research participants.

\subsection{Triangulation}

Johnson and Christensen (2008) defined triangulation as “... term given when the researcher seeks convergence and corroboration of results from different methods studying the same phenomenon" (p.451). Triangulation means using more than one data collection method (Denscombe, 1999; Neuman, 1997; Stake, 1995). Combining multiple data collection methods (Denscombe, 1999; Denzin \& Lincoln, 2000; Dubé \& Paré, 2003; Golafshani, 2003; Neuman, 1997; Pickard, 2007; Stake, 1995), the researcher overcame the weaknesses or intrinsic biases and problems that might arise from using a single data collection method. Neuman (2011) stated that "... looking at something from multiple points of view improves accuracy" (p.164).

Within-method type of triangulation could be used for multiple complementary methods are used (Hussein, 2009). In this research the multiple data collection methods were interviews, focus group discussions and a qualitative survey. The researcher used data collection multiple methods within the qualitative paradigm towards increasing credibility, completeness and to confirm of findings through convergence of different perspectives. Use of multiple data collection methods can be used for completeness purposes (Hussein, 2009). In addition, the researcher aimed to achieve validity of findings by adopting the triangulation method.

\subsection{Chapter Summary}

This chapter identified the research methodology adopted for this study. The principal methodology is best described as a case research methodology, where the Maldives is considered as the main Case. The chapter began by outlining the philosophical background of the research with an introduction to the qualitative approach.

The chapter explained the design considerations with the type of data required; how the data was collected and discussing about the unit of analysis. The data 
collection methods were interviews, focus group discussion, qualitative survey and document reviewing.

The chapter concluded by highlighting the qualities of qualitative research (i.e. credibility, transferability, dependability and confirmability). The rigour of the research, ethical considerations, confidentiality and anonymity were discussed. The concept of data collection conditions and triangulation were explained in the last sections of this Chapter.

The next chapter provides the background to the specific case of the Maldives and explains the geography, culture, education system, ICT, and main characteristics of ICT in higher education. 


\section{CHAPTER 4}

\section{CASE OF THE MALDIVES}

\subsection{Introduction}

This chapter explains the case of the Maldives where the research was undertaken. It starts with an overview of the Maldives and its geography. The chapter gives a description of the Maldives' economy, population densities in the islands, and system of governance. It also explains the culture of the Maldives, including its Islamic dimensions and gender aspects.

The chapter highlights the education system in the Maldives, which includes primary education, secondary education and higher education. Finally, the chapter concludes by describing the ICT in the Maldives, with special emphasis on the existence of ICT infrastructures and ICT projects. A comparison is made between the Maldives and other South Asian Regional Countries, together with SIDS.

\subsection{The Maldives and geography}

\subsubsection{The Maldives}

The Maldives is an archipelago comprising 1,192 coral islands across the Indian Ocean over an area of 850 square kilometres, grouped into 20 atolls ${ }^{1}$ with 198 inhabited islands (Country Report, 2009; InfoDev, 2010a; Minges \& Gray, 2004; Reddi \& Sinha, 2005; Shareef et al., 2010). The Maldives is among the 52 SIDS countries listed by United Nations (UN), consistent with the criteria of "islandness", "stateness", and "smallness" (UNCTAD, 2004b). The World Bank (2000) report in particular highlighted that remoteness and natural disasters such as tsunami pose considerable risks and challenges to the Maldives.

Only a few SIDS such as the Maldives have hundreds of small, inhabited islands where basic services have to be delivered. Most islands are economically

\footnotetext{
${ }^{1}$ An atoll is a group of islands. The Maldives has 26 natural atolls, which, for administrative purposes, are divided into 20 atolls.
} 
dependent on the capital, Male' (Ibrahim, 2006), and people must travel by traditional boat (i.e. dhoani ${ }^{2}$ ), which is time consuming (Infodev, 2010a).

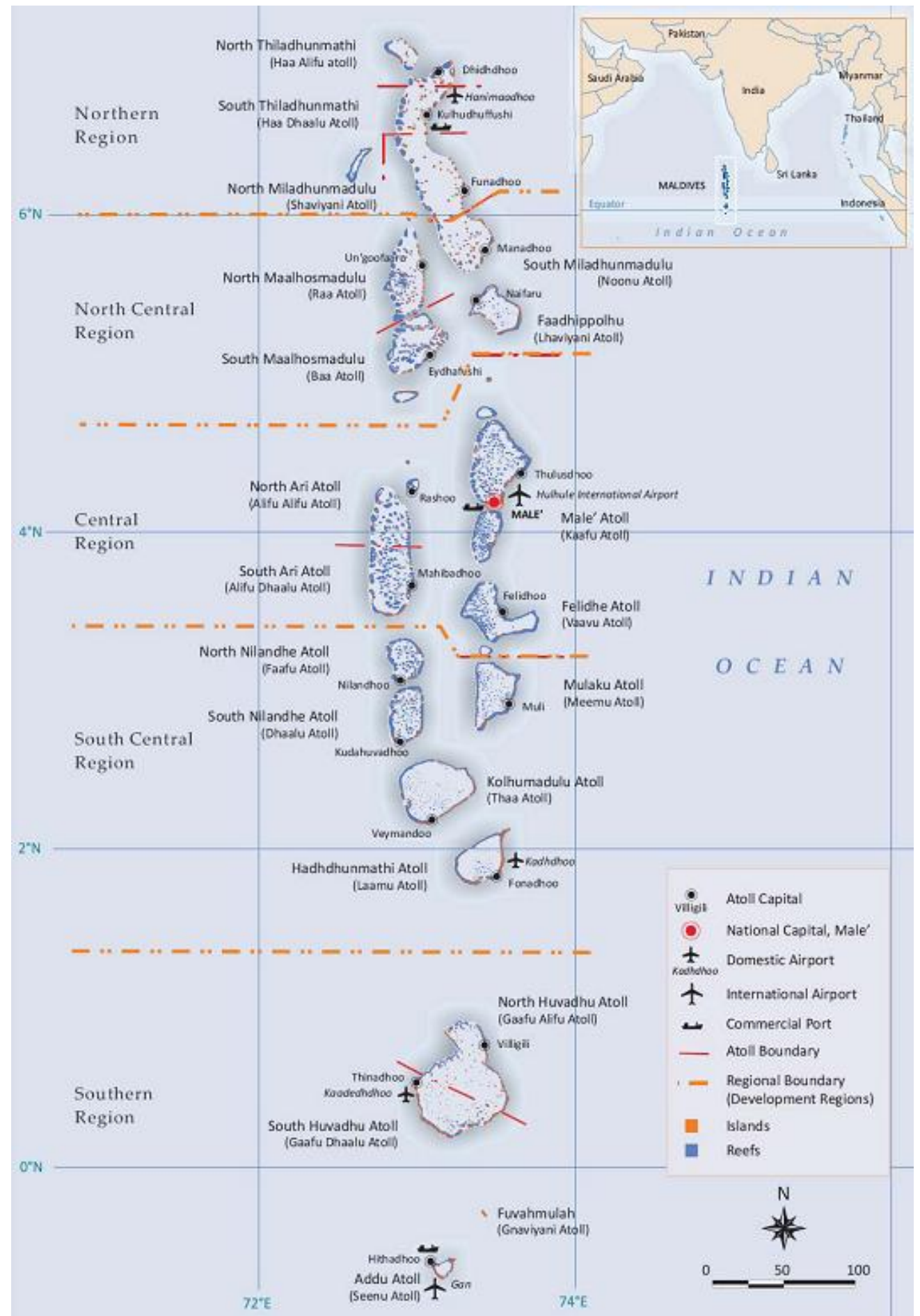

Figure 10: Map of the Maldives

Source: Department of National Planning (2011, p.vii)

${ }^{2}$ Dhoani is a traditional fishing vessel, also used as inter-island ferries throughout the Maldives. 
Figure 10 shows a map of the Maldives with all 20 atolls. The word "atoll" was derived from the national language of the Maldives, called Dhivehi. ${ }^{3}$ In the Dhivehi language, the word atoll is "atholhu". The atolls can vary widely in size, from a single island of a few square kilometres to large blue lagoons with diameters of more than 70 kilometres (Country Report, 2009).

The islands are small, and a large proportion of the islands have an area of less than half a square kilometre and only a few are bigger than two square kilometres (Shareef et al., 2010). Due to the geographical area there are many logistical challenges in delivering higher education services to all the islands of the Maldives.

The Government of the Maldives has categorised the 20 atolls into seven administrative provinces (Decentralisation Act, 2010). These provinces are each headed by a State Minister in order to govern the development of local government and empower citizens at local levels (Decentralisation Act, 2010). The Government envisions the establishment of a three-tier governance structure: a) Ministries at urban level; b) Province Offices and Atoll Offices at regional level; and c) Island Offices at island level with decentralised functions under elected councils (Decentralisation Act, 2010).

The oceanic environment of SIDS creates problems such as natural disasters (Koonjul, 2004). Arguably most SIDS are subject to natural disasters of some form such as typhoons and tsunamis, which are accentuated by global warming. Global warming and sea level rises have consequently become major environmental concerns in the Maldives (Country Report, 2009). Sea-level rise estimates of 18-59 cm by year 2100 would be dire for SIDS such as Tuvalu, Marshall Islands, Micronesia, and the Maldives (Wong, 2011).

The Government of the Maldives has articulated a vision for an information society that emphasises the notion of widespread access to technology that can nurture human capital and provide better education for all citizens (Department of

${ }^{3}$ Dhivehi Language the national language of the Maldives. The script of Dhivehi Language is called "Thaana". 
National Planning, 2011). The National Development Plan envisaged that effective use of technology has the potential to revolutionise the higher education system within the Maldives (Department of National Planning, 2011).

Despite having a growing number of young people, the Maldives faces a significant challenge in harnessing and promoting higher education facilities in urban and rural areas. There is no critical mass of population in these small islands to support expensive higher education delivery through conventional Higher Education Institutes (HEI).

In addition, it would be impractical and uneconomical for the Maldives to establish tertiary institutes on every island. An obvious response is to make better use of existing ICT within the higher education sector and to distribute higher education services through ICT. This would allow the delivery of higher education services to remote islands as well as urban populations in Male'. The Maldivian Government is aware that increasing access to tertiary education is important for improving its human resources, which is an important element in the country (Country Report, 2009).

\subsubsection{Geography}

The Maldives is an independent SIDS with a small economy. However, the Maldives like other SIDS has problems in terms of income vulnerability and lack of opportunity, often exacerbated by external economic shocks and natural disasters. SIDS such as the Maldives have development challenges due to sea level rise and global climate change (Wong, 2011).

Geographical remoteness in rural areas counts as a barrier for higher education service delivery (Lane, 2009). The low population in the islands makes the Maldives distinctive, even among small island archipelago states in the world.

Even the tourism sector is heavily dependent on expatriate workers on all levels due to lack of human capital in the Maldives (Adam \& Urquhart, 2007). Expatriate 
workers (Ibrahim, 2010) make up about one fourth of the labour force (Minges \& Gray, 2004).

\subsubsection{Transportation}

Table 3 illustrates the diverse population numbers of the individual capital atoll islands. ${ }^{4}$ Table 3 shows the maximum distance to Male' from the most distant island within each atoll and the maximum distance to each atoll's capital island.

Table 3 show the great distances between islands, which means the islanders have to spend a lot of money in travel between islands and Male'. The transport costs between the islands are extremely high and $80 \%$ of teachers' training costs involve transportation (InfoDev, 2010b). Transportation is difficult and accessibility of resources has become a problem (Aneh Dhivehi Rajje, 2009-20135).

The IT service providers are unwilling to provide network services due to travelling costs, time, money and lack of return on investments. The delivery of services is difficult due to the unavailability of regular transport between islands (Aneh Dhivehi Rajje, 2009-2013). The geographical situation of the Maldives raises the cost of delivering education (Koonjul, 2004; Shareef \& Kinshuk, 2002; WHO, 2006).

\begin{tabular}{|c|l|c|l|c|c|}
\hline No & \multicolumn{1}{|c|}{ Atolls } & $\begin{array}{c}\text { Population } \\
\text { of an atoll }\end{array}$ & $\begin{array}{c}\text { Capital Atoll } \\
\text { Island }\end{array}$ & $\begin{array}{c}\text { Maximum Distance } \\
\text { to Male' from the } \\
\text { most distant island } \\
\text { within atoll (in km) }\end{array}$ & $\begin{array}{c}\text { Maximum Distance } \\
\text { to Capital Atoll } \\
\text { within atolls (in } \\
\text { km) }\end{array}$ \\
\hline 1 & Haa. Alifu & 13,314 & Dhidhdhoo & 332.6 & 33.3 \\
\hline 2 & Haa Dhaalu & 16,214 & Kulhudhufushi & 294.9 & 20.3 \\
\hline 3 & Shaviyani & 11,830 & Funadhoo & 260.2 & 52.4 \\
\hline 4 & Noonu & 10,015 & Manadhoo & 201.5 & 25.2 \\
\hline 5 & Raa & 14,643 & Un'goofaaru & 208.5 & 34.2 \\
\hline 6 & Baa & 8,893 & Eydhafushi & 134.5 & 28.6 \\
\hline 7 & Lhaviyani & 8,346 & Naifaru & 147.0 & 31.9 \\
\hline 8 & Kaafu & 10,149 & Thulusdhoo & 0 & 31.9 \\
\hline 9 & Alifu Alifu & 4,855 & Rasdhoo & 90.2 & 47.5 \\
\hline 10 & Alifu Dhaalu & 6,921 & Mahibadhoo & 110.8 & 36.4 \\
\hline 11 & Vaavu & 1,502 & Felidhoo & 95.5 & 27.2 \\
\hline
\end{tabular}

${ }^{4}$ For easy governance each atoll has a capital atoll island

${ }^{5}$ The Strategic Action Plan 2009-2013 will serve as the principal planning document of the government in the delivery of the Government's pledges and programmes outlined in the Maldives Democratic Party Alliance Manifesto. 


\begin{tabular}{|c|l|c|l|c|c|}
\hline No & \multicolumn{1}{|c|}{ Atolls } & $\begin{array}{c}\text { Population } \\
\text { of an atoll }\end{array}$ & $\begin{array}{c}\text { Capital Atoll } \\
\text { Island }\end{array}$ & $\begin{array}{c}\text { Maximum Distance } \\
\text { to Male' from the } \\
\text { most distant island } \\
\text { within atoll (in km) }\end{array}$ & $\begin{array}{c}\text { Maximum Distance } \\
\text { to Capital Atoll } \\
\text { within atolls (in } \\
\text { km) }\end{array}$ \\
\hline 12 & Meemu & 4,654 & Muli & 155.1 & 23.3 \\
\hline 13 & Faafu & 3,662 & Nilandhoo & 141.9 & 26.8 \\
\hline 14 & Dhaalu & 4,720 & Kudahuvadhoo & 180.4 & 38.3 \\
\hline 15 & Thaa & 8,451 & Veymandoo & 220.8 & 43.2 \\
\hline 16 & Laamu & 11,743 & Fonadhoo & 266.5 & 32.7 \\
\hline 17 & Gaa Alifu & 8.007 & Viligili & 424.4 & 46.3 \\
\hline 18 & Gaa Dhaalu & 10,991 & Thinadhoo & 443.1 & 57.4 \\
\hline 19 & Ghaviyani & 7,636 & Fuvahmulah & 497.0 & 0 \\
\hline 20 & Seenu & 17,862 & Hithadhoo & 541.1 & 15.9 \\
\hline
\end{tabular}

Table 3: Atolls populations and distance to Male'

Source: Department of National Planning, 2011; Department of National Planning, 2010b; Shareef, 2005

The UNCTAD (2004b) report stated that: “ ... in the early UNCTAD and United Nations General Assembly resolutions of the 1970s and 1980s on island developing countries, the problems associated with smallness, lack of natural resources, heavy dependence on imports, limited internal markets, export concentration" (p.7).

Koonjul (2004) explained that developing countries, both SIDS and non-SIDS, all face substantial challenges in their development paths. Nevertheless, the common mother tongue (Dhivehi) and religion (Islam) have been two important factors that unite the Maldivian people into a cohesive and peaceful society (MTCA, 2008; MPND, 2006).

The International Telecommunication Union pointed out that, due to the small and dispersed islands in the Maldives, the digital divide and technology gap pose a major challenge (ITU, 2004). The disproportionate growth of the capital Male' with respect to the other atolls is an issue of major concern. Reddi and Sinha (2005) described the disparity of ICT in the Maldives as "disparity in the accessibility of ICT between urban and rural areas" (p.256).

The digital divide is a gap that exists between the wired rich living in Male' and the limited or unconnected poor on the islands. The digital divide is because of numerous small island communities geographically distributed across a vast sea area (ITU, 2004). 
The geographic and demographic natures of the islands are challenges to the national development of the Maldives (Shareef et al., 2010). Reducing this digital divide is a major governmental challenge, caused by the difficulty of providing services to small and dispersed locations (Minges \& Gray, 2004).

It is important to increase the number of people who have access to and use the technology to raise the level of digital inclusion (Herselman \& Britton, 2002). These remote islands depend largely on the capital for services and islanders travel to the capital for higher education services. The islanders are particularly isolated because of the distance and areas of sea between the many islands and atolls (Reddi \& Sinha, 2005).

\subsubsection{Capital - Male' and remote islands}

The capital of the Maldives is Male'. This is the largest city with almost one third of the population (Department of National Planning, 2011; Shareef et al. 2010; Department of National Planning, 2010a; Department of National Planning, 2010b) of this total lives in Male' an area of less than 2 sq. km (Department of National Planning, 2010b; MPND, 2006).

Due to overcrowding, housing has been an issue in Male'. The Human Rights Commission of Maldives report found that around 12,000 families in Male' do not have their own housing and 70 per cent live fails to meet "adequate housing" criteria (HRCM, 2009).

Figure 11 illustrates Kaafu atoll where capital Male' is located. This figure shows the islands and reefs within an atoll with deep sea around. Travelling between the islands is difficult due to lack of available transportation and impossible in bad weather. 


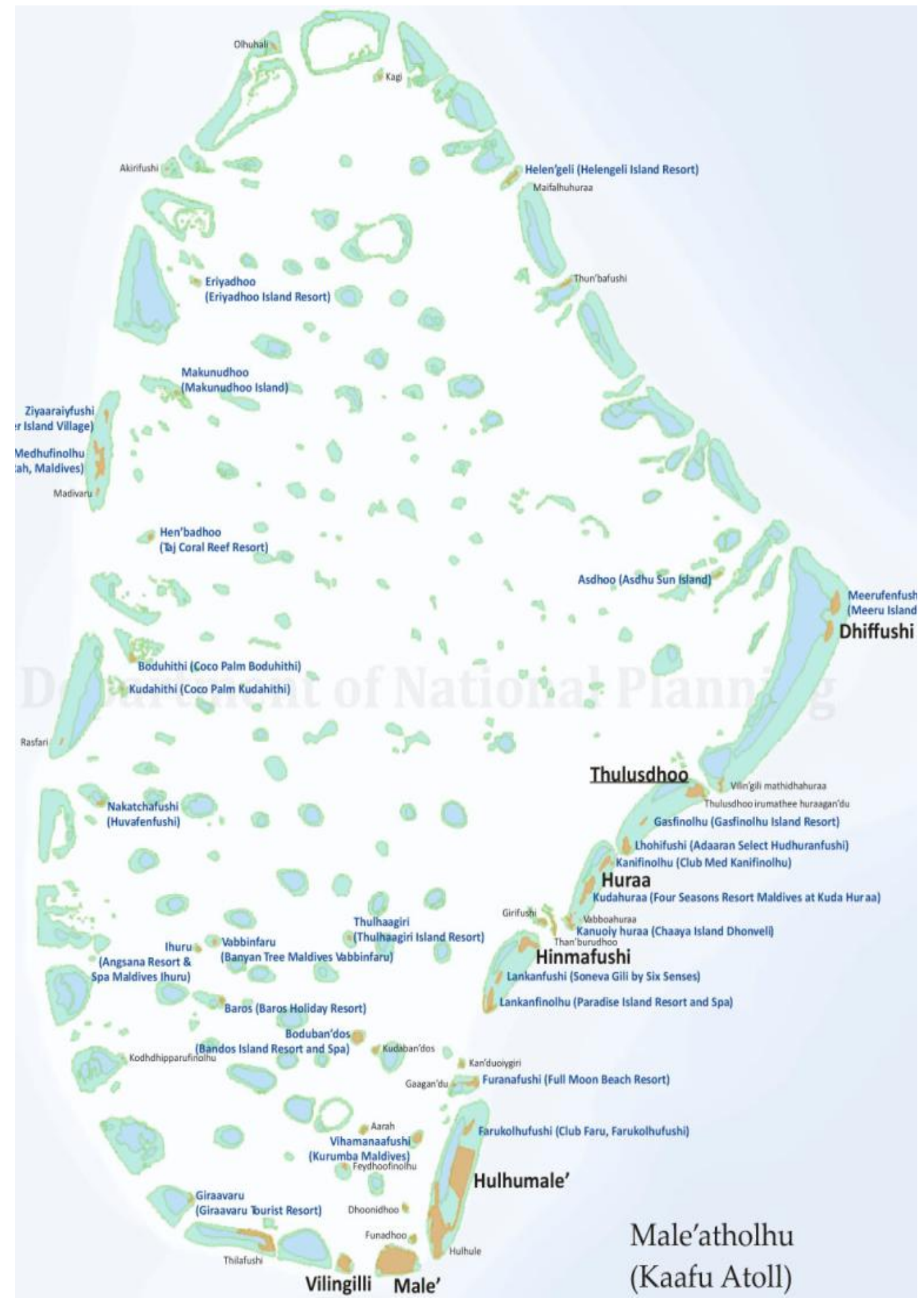

Figure 11: Kaafu atoll in the Maldives

Source: Department of National Planning, 2012c

\subsection{Maldives' economy, population and governance}

The economy, population and governance are three important aspects to consider in the context of the Maldives. The economy is primarily based on tourism. The population is small compared to other regional countries, but not the lowest 
among SIDS. The governance is a four-tiered architecture: central government, city council, atoll council, and island council.

\subsubsection{Economy}

The economy of the Maldives is stable and based on two major activities - tourism and fishing (Minges \& Gray, 2004). The Maldives is a popular tourism destination in the world (Henderson, 2008; MTCA; 2008). The annual number of tourist arrivals is almost three times greater than the entire population of the Maldives (Department of National Planning, 2010a; Department of National Planning, 2010b).

According to the Department of National Planning (2012b) key economic indicators, the latest Gross Domestic Product (GDP) per capita grew between 1978 (US\$300) to 2012 (US\$3936). In 2005, the Maldives had the fastest growing economy in South Asia with an annual growth rate of 6\% (MPND, 2006).

\subsubsection{Population}

According to the Department of National Planning (2012a), the latest estimated population figure is 350,759 with almost $51 \%$ male and $49 \%$ female inhabitants. The Maldives has a young population with 45\% aged between 6 to 20 years (Department of National Planning, 2012a). With the increase in the young population, special attention is being given by local educational authorities to expansion of higher education to all regions. Table 4 depicts the number of islands in terms of with population size. It is economically impossible to deploy basic physical higher education infrastructure in all these islands.

\begin{tabular}{|c|c|}
\hline Population Size & $\begin{array}{c}\text { No of } \\
\text { islands }\end{array}$ \\
\hline Less than 500 & 95 \\
\hline $500-900$ & 47 \\
\hline $1000-1499$ & 42 \\
\hline $1500-4999$ & 11 \\
\hline $5000-9999$ & 2 \\
\hline 10000 and over & 1 \\
\hline
\end{tabular}

Table 4: Number of islands by size of population

Source: Department of National Planning, 2011 
Among the 198 inhabited islands, almost one third have a population of less than 500 , and $70 \%$ have a population of less than 1,000 (Department of National Planning, 2012a; Department of National Planning, 2011; Shareef et al., 2010). There are about 95 islands with less than 500 people and some islands have only 30-50 inhabitants (Department of National Planning, 2012a; Department of National Planning, 2011; Shareef, 2005). According to Ibrahim (2006), the population density varies across the Maldives and only six islands have more than 3000 inhabitants. Due to the small populations there are few people working, which creates diseconomies of scale that hinder the development of the country (Department of National Planning, 2011; InfoDev, 2010a; Shareef et al., 2010).

\subsubsection{Employment}

Demographically, the proportion of young unemployed males is growing which is made worse by the lack of opportunities and higher education within the Maldives (Henderson, 2008). Creating employment opportunities, particularly for secondary school leavers, is a major challenge for any Government in the Maldives. Considerable resources have been invested in human resource development in recent years, which is under the Seventh National Development Plan with a greater focus on youth employment (Department of National Planning, 2011).

The use of drugs, lack of educational opportunities for youth and boredom have led to a social crisis and an increase in crime within the Maldivian community (Fulu, 2007). Henderson (2008) believed that problems such as drug abuse are issues that need to be addressed urgently by the Government of the Maldives.

The dilemma of drug addiction has touched almost all families in the Maldives. According to the Narcotics Control Board (2006), "... drug abuse has become more open and visible on Male streets and even some cafes (p.16). This statement provides a clear reflection of the seriousness of the issues, which is a huge challenge for all the families. Ageel's (2006) study raised concern that current practices were neither implemented nor enforced in the Maldives but merely a piece of paper. 


\subsubsection{Governance System}

According to the Decentralisation Act (2010), the Maldivian governance system is a democracy and has four levels:

- Central Government: The central Government is the capital city with all the powers.

- City Council: There are two cities in Maldives: Male' city (Male' atoll) and Addu city (Seenu atoll). Male' city is in the middle and Addu city in the south of the Maldives. A city is an inhabited area with a population of at least 25,000 people with a "city council" governing.

- Atoll Council: An atoll council has the power to govern within the atoll. Each twenty administrative atolls have an "atoll council" which monitors the work of the "island councils" in the atoll, and maintains communications with the central government.

- Island Council: An island council has the power to govern within the island. Island refers to a land-mass surrounded by water.

\subsection{Culture}

The culture includes the Maldives' Islamic dimensions and employment. As part of culture, the gender aspect is considered here as an important element when it comes adopting ICT into higher education.

\subsubsection{Islamic Dimensions}

Ethnically Maldivians are Dravidian and, while their origins go back to different groups, they are predominantly of Indian and Sri Lankan descent (Minges \& Gray, 2004). The Maldives converted to Islam in the $12^{\text {th }}$ century (Duch, 2005) and the country is fully Islamic with Islam as the official religion (Cambridge Education, 2005; Duch, 2005; Fulu, 2007). 
Public observance of religion other than Islam is strictly prohibited in the Maldives. Islam also strictly prohibits drugs and alcohol consumption. Alcohol can be consumed only by foreigners in the resorts of the Maldives. Islam has a strict dress code and basic decency in dress is expected.

According to the constitution of the Maldives, every citizen of the Maldives should follow Islam as their religion (D. Hussain, 2008). Muslims pray to Allah five times a day, responding to the calls over the loud speakers of the many mosques within the Maldives. The significance and importance of Islam flow through all aspects of life and are given high priority in teaching and learning. The Holy Quran strictly guides the teaching and learning concept as the first five verses revealed by Allah were about teaching and learning.

Fulu (2007) believed that, although the Maldives is a geographically dispersed island nation, the country is connected through bonds of religion and language (Minges \& Gray, 2004), which makes it distinct from the other countries in the region (Cambridge Education, 2005). The Dhivehi language is only spoken by the people of the Maldives. The script of Dhivehi language is called "Thaana" and can be represented by computers. While the official language is Dhivehi, English is widely spoken and the de facto language of instruction in education (Cambridge Education, 2005).

\subsubsection{Gender}

Although there is no official gender discrimination in the Maldives, females have fewer opportunities for jobs and education. Females in developing countries are disadvantaged with respect to males (Looker, 2008; Elnaggar, 2008). There is also a gender imbalance in the labour force where Maldivian women are heavily underrepresented (Henderson, 2008). In the case of ICT use, Looker (2008) noted that females in the developing world have less access to ICT and lower rates of IT use.

Ainley, Enger and Searle (2008) observed gender differences in ICT use even in developed countries, with males using the Internet more than females. This situation is the same in other regions of the world where women's access and 
utilisation of IT within the Arab states continue to lag behind that of men, which creates a widening gender digital divide (Elnaggar, 2008). Research undertaken in the Pacific Islands showed that women are more likely to get higher education if they can access distance learning, which allows them to stay with their families (UNESCO, 2011).

\subsection{Education System}

The education in the Maldives today is directly connected to the traditional system of schooling that has existed for centuries. Higher Education is provided by the Maldives College of Higher Education6, private colleges and other institutions which offer certificates, diplomas, degrees, masters, doctorate in courses in a range of disciplines.

Aneh Dhivehi Rajje (2009-2013), which highlighted the national education strategies for 2009-2013, has three points which are worthy to note: a) to facilitate use of relevant Internet resources for teaching and learning; b) to expand equitable access to ICT facilities; and c) to develop and facilitate use of appropriate ICTs in the administration and management of schools.

\subsubsection{School System}

There are two school systems in the Maldives (i.e. traditional and western style). Students usually attend both school systems.

The traditional school system in the Maldives comprises three types of schools: Edhuruge, makthab and madhrasa (UNESCO, 2010; Pillay \& Elliot, 2005). These are privately owned or operated by the island communities and are usually selffinancing (UNESCO, 2000). Traditionally children aged three and upwards in the Maldives were educated in Islamic "Makthab" classes or "Edhuruge", generally using a single large room or the shelter of a tree (Duch, 2005).

The "Edhuruge" concept entails Holy Quranic schools which teach the Holy Quran, basic arithmetic, and the ability to read and write Dhivehi (UNESCO, 2000;

\footnotetext{
${ }^{6}$ Now it is called The Maldives National University. When the researcher collected the data it was called Maldives College of Higher Education
} 
UNESCO, 2010). Primary-school children attend "Edhuruge" to gain extra tuition in Quran recitation.

Madhrasa schools offer a wide curriculum, which includes more subjects such as literacy skills, and have contributed towards achieving high rate of literacy (UNESCO, 2000). The modern teaching involves primary and secondary schooling with Dhivehi, Islam, and the Quran as subjects (UNESCO, 2000).

The Maldives secondary school system follows the British system of education (Duch, 2005; Minges \& Gray, 2004). The Maldives became a British protectorate in 1887 (Duch, 2005). The Maldives gained political independence from Britain on the 26 $6^{\text {th }}$ July 1965 (Duch, 2005; Minges \& Gray, 2004). The British education system was adopted in 1960 by the Government of the Maldives. The western style of schooling was introduced to the Maldives to prepare individuals to meet the increasing developmental needs (UNESCO, 2000).

There is no direct relationship between the traditional school system and the British education system (UNESCO, 2000). The London EdExcel syllabus is followed in the secondary schools, which allows the students to sit for exam-based assessments.

\subsubsection{Higher Education in the Maldives}

The higher education sector has been important to the Maldives over recent decades (Department of National Planning, 2011). The success of school system in the Maldives in the last ten years has intensified the demand for higher education (Waheed, 2009). This view is expressed in the Maldives Seventh National Development Plan (NDP), which stated "strengthening and expansion of higher education and training is central to achieving the prime objectives of reducing vulnerability and poverty" (Department of National Planning, 2011, p.134).

It is estimated that almost one third of the population of the Maldives are studying in primary and secondary schooling or tertiary education (MOE, 2012). Over the 
last decade there has been an increased number of secondary school leavers (i.e. lower secondary and higher secondary).

\begin{tabular}{|c|c|c|}
\hline Year & $\begin{array}{c}\text { No of school } \\
\text { leavers lower } \\
\text { secondary (Grade } \\
\mathbf{8 - 1 0} \text { schooling) }\end{array}$ & $\begin{array}{c}\text { Number of school } \\
\text { leavers in higher } \\
\text { secondary (Grade } \\
\mathbf{1 1 - 1 2} \text { schooling) }\end{array}$ \\
\hline 2004 & 26141 & 1622 \\
\hline 2005 & 28646 & 1942 \\
\hline 2006 & 29084 & 2214 \\
\hline 2007 & 29417 & 2486 \\
\hline 2008 & 28164 & 2651 \\
\hline 2009 & 26120 & 2920 \\
\hline 2010 & 24588 & 3243 \\
\hline 2011 & 22788 & 4413 \\
\hline
\end{tabular}

Table 5: Secondary School Leavers (2004 to 2011) Source: MOE, 2012

Table 5 shows the secondary school leavers from 2004 to 2011. An increase in number of school leavers does not necessarily means they should go into higher education. In fact, the higher education institutes are unable to cater for the increased number of secondary school leavers. There is a lack of jobs for the school leavers since most jobs require higher-level educational qualifications.

Therefore, the need for higher education is important for the Government and people of the Maldives. Higher education will be always be a priority for any country, and this need is clearly stated in the Maldives NDP “... strengthening and expansion of higher education and training is central to achieving the prime objectives of reducing vulnerability and poverty in Maldives" (Department of National Planning, 2011, p.134).

The UNESCO conference paper (Country Report, 2009) presented by the Maldivian delegate stated that the "... Maldivian Government recognises that increasing access to tertiary education is important for improving its human resource base - ultimate the nation's most important resource" (p.2.) 


\begin{tabular}{|l|c|}
\hline \multicolumn{1}{|c|}{ Institutes } & \multicolumn{1}{|c|}{$\begin{array}{c}\text { Estimated } \\
\text { number of } \\
\text { students } \\
\text { studying }\end{array}$} \\
\hline $\begin{array}{l}\text { Maldives College of Higher Education (public) - current name The Maldives } \\
\text { National University }\end{array}$ & 4095 \\
\hline Villa College (private) & 608 \\
\hline Mandhu College (private) & 472 \\
\hline Cyryx College (private) & 2557 \\
\hline Modern Academy for Professional Studies (MAPS) College (private) & 237 \\
\hline Clique College (private) & 757 \\
\hline Focus Education Centre (private) & 130 \\
\hline
\end{tabular}

Table 6: Student numbers at seven higher education institutes

Source: $\quad$ World Bank, 2011; MNU, 2011

Higher education in the Maldives is delivered by a wide range of institutions both public and private (World Bank, 2011). Table 6 shows the total number of students in the public and private higher education institutes based in Male'. There is one large public higher education institute, Maldives College of Higher Education $(\mathrm{MCHE})^{7}$, with several private registered institutes operating in the Maldives.

The MCHE was established to improve the tertiary education in the Maldives (Country Report, 2009; World Bank, 2011). Maldives College of Higher Education is the only public degree-granting public institution in the Maldives (Cambridge Education, 2005; Mohamed, 2005). The higher education system in the Maldives (i.e. degree or above and pre-degree level) has an estimated total enrolment of about 11,000-12,000 students (World Bank, 2011).

The Maldives National University was launched on 15 th February 2011 (MNU, 2011). The opening of the national university has allowed students to have more quality tertiary level educational opportunities. The concept of higher education opportunities is stated in National Development Plan Policy 1 of the higher education chapter as "expand and increase higher education opportunities" (p.135). However, the higher educational opportunities are limited and the World Bank

\footnotetext{
${ }^{7}$ Maldives College of Higher Education (MCHE) was renamed The Maldives National University (MNU) on $15^{\text {th }}$ February 2011. When the researcher collected data in May to September 2009, the institution was known as MCHE. For consistency, the researcher has used MCHE throughout the thesis.
} 
(2011) report stated, "Higher education enrolment in the Maldives is low for a middle-income country" (p9).

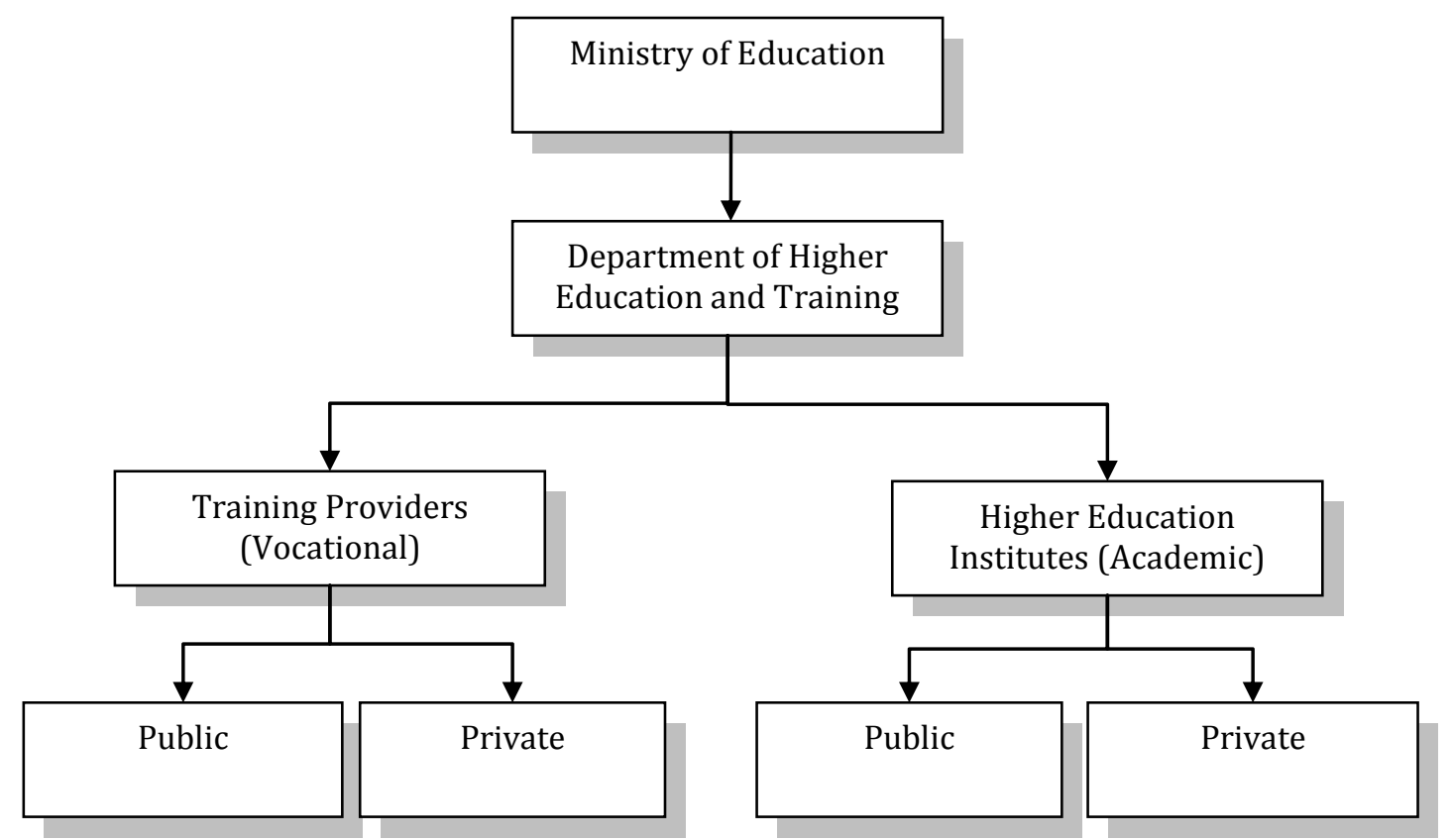

Figure 12: Higher Education System in the Maldives Source: MQA, 2009

Figure 12 illustrates the hierarchy of the higher education system in the Maldives. It is a top-down approach where the highest authority for education is the Ministry of Education. The Maldives Government has now a central role to play in strengthening and expanding higher education and training to achieve the national development objectives (Country Report, 2009; World Bank, 2011).

\subsubsection{Maldives National Qualification Framework (MNQF)}

The Maldives Qualifications Authority (MQA) is responsible for managing the Maldives National Qualifications Framework (Country Report, 2009). The main objective of the MNQF was "strengthened MNQF is the unified qualifications system which guarantees more international recognition for the qualifications and the skills and knowledge of Maldivian citizens" (MQA, 2009, p.4).

Figure 13 shows the Maldives National Qualifications Framework (MNQF) framework has 10 levels of qualifications including Certificate 1, Certificate II, Certificate III, Advanced Certificate, Diploma, Advanced Diploma, Advanced 
Diploma, Bachelors degree, Master's degree and Doctoral degree (MQA, 2009, Mohamed, 2005; UNESCO, 2010).

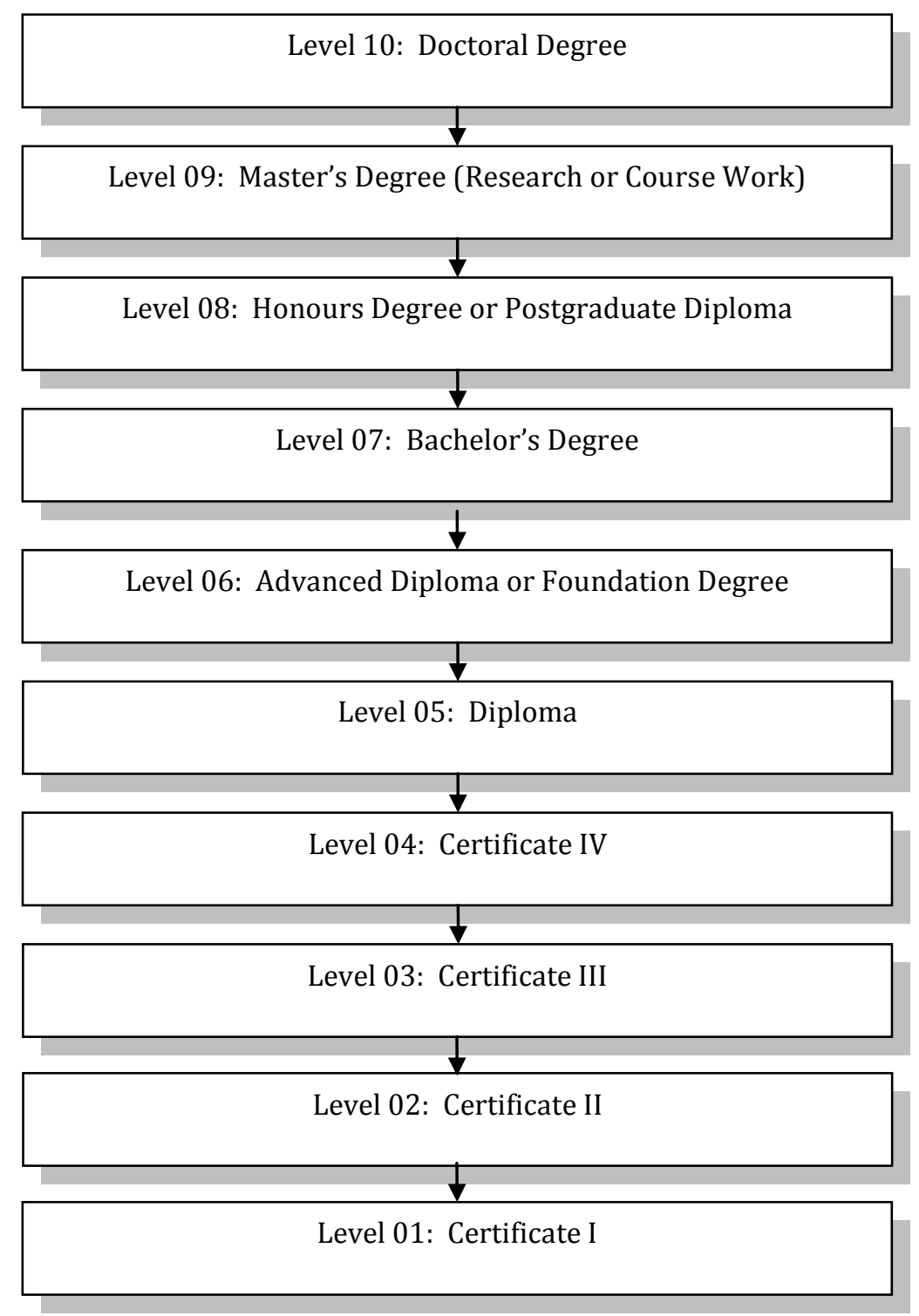

Figure 13: Maldives National Qualification Framework (MNQF)

Source: $\quad$ MQA, 2009; World Bank, 2011; UNESCO, 2010

The MNQF provides a coherent framework that facilitates quality improvement, quality assurance, and higher education (MQA, 2009). The MNQF ensures that students, employers, institutes understand comprehend the learning outcomes involved in various qualifications (Country Report, 2009; Mohamed, 2005). 


\subsection{Information and Communications Technology (ICT)}

The National Development Plan highlighted the importance of expanding and promoting ICT in higher education through reduction of Internet charges and improved connectivity (Department of National Planning, 2011). National Development Plan has emphasised greater use of ICT in the school curriculum, creating ICT-based institutes of higher education and expanding the use of distance learning in the Maldives (Department of National Planning, 2011). The technologydriven methodologies have potential in higher education. Ibrahim (2006) stated that "e-Government, distance learning, telemedicine and e-commerce also have enormous potential in this nation of atolls" (p.167).

\subsubsection{ICT in the Maldives}

The Government of the Maldives appreciates the benefits that can be gained from ICT in all spheres of the rapidly growing sector of ICT education (Department of National Planning, 2011; Reddi \& Sinha, 2005). Educational processes are moving into a virtual world, which allows $24 / 7$ access from any location (Loxley \& Julien, 2004). In 1999, the former President of the Maldives introduced a programme to make all students "Computer Literate". The need for ICT-based higher education is regarded as a key priority by the Maldives Government (Department of National Planning, 2011; MCHE, 2008).

\begin{tabular}{|l|l|c|}
\hline \multicolumn{1}{|c|}{ Type } & \multicolumn{1}{c|}{ Activity } & Amount \\
\hline Demographics & Population (2012 figure) & 350,759 \\
\hline & Gross Domestic Product (GDP) & US\$2,900 \\
\hline Fixed Telephone lines & Total number of telephone lines (includes payphones) & 48,056 \\
\hline & Other inhabited islands & 12,509 \\
\hline & Tourist Resorts & 980 \\
\hline Mobile & Total Number of registered mobile phones & 540,004 \\
\hline & Mobile subscriptions - Post-paid & 77,652 \\
\hline & Mobile subscriptions - Pre-paid & 462,352 \\
\hline Tele-density & Overall Teledensity - Fixed lines & 15.03 \\
\hline & Overall Teledensity - Fixed lines \& Mobile lines & 168.89 \\
\hline Internet & Internet subscription customers & 1,323 \\
\hline & ISDN lines & 1,219 \\
\hline & ADSL lines & 10,195 \\
\hline & Leased lines & 46 \\
\hline & Internet users & 107,460 \\
\hline & Internet penetration rate (\% population) & $28.9 \%$ \\
\hline & Number of computers (estimated) & 40,000 \\
\hline & V-Sat Links (20 atolls) TRC & 20 \\
\hline
\end{tabular}

8 Telecommunications penetration expressed as a percentage of population 


\begin{tabular}{|l|l|c|}
\hline \multicolumn{1}{|c|}{ Type } & \multicolumn{1}{|c|}{ Activity } & Amount \\
\hline & Submarine cables & 4 \\
\hline & Internet Service Providers (ISP) & 2 \\
\hline Card phones & Total number of public and card phones & 371 \\
\hline & Card phones in capital & 98 \\
\hline & Card phones in other islands & 337 \\
\hline & Private card phones & 50 \\
\hline Radio \& TV & Radio Stations & 4 \\
\hline & TV stations & 2 \\
\hline
\end{tabular}

Table 7: ICT in the Maldives

Source: CAM, 2013; Department of National Planning, 2011; ITU, 2010; Internet World Stats, 2013

Table 7 illustrates some basic statistics about ICT in the Maldives. The ICT include Internet connectivity, mobile penetration, card phones, radio and TV. The latest figures from Internet World Stats (2013) showed that the Internet penetration figures are even more promising for the Maldives, since there are now more than 107,460 Internet users, with an Internet penetration rate of 28.9\% (Internet World Stats, 2013).

The statistical figures provided by International Telecommunication Union showed that the Internet penetration in the Maldives ranks at number one among South Asian countries, second among the Least Developed Countries and 14th in SIDS (InfoDev, 2010a; ITU, 2010; UNCTAD, 2009; UNESCAP, 2008). With these promising statistics, the Maldives now should find ways to use the available ICT existing resources within the higher education system and also in other sectors.

There is complete telecommunication coverage with different modes of communication in the Maldives, making international communication very easy (MTC, 2006; TAM, 2011). The Maldives has good telecommunication coverage compared to other neighbouring countries in the region (Asian Development Bank - ADB, 2010; ITU, 2010; TAM, 2011; UNCTAD, 2009) and among SIDS (ITU, 2010). Virtually all islands offer all telecommunication services, from international direct dial phone and fax service to Internet access (TAM, 2011).

The Maldives now has more mobile subscriptions, both pre-paid and post-paid, than the entire population (TAM, 2011; UNCTAD, 2009). These levels make 
Maldivians the highest mobile subscribers in the South Asia region (ITU, 2010; UNCTAD, 2009).

Figure 14 illustrates the percentage of access to the Internet per 100 inhabitants in the Maldives from 1995 to 2011. This figure shows there was sharp increase in Internet users over the past year except for 2010.

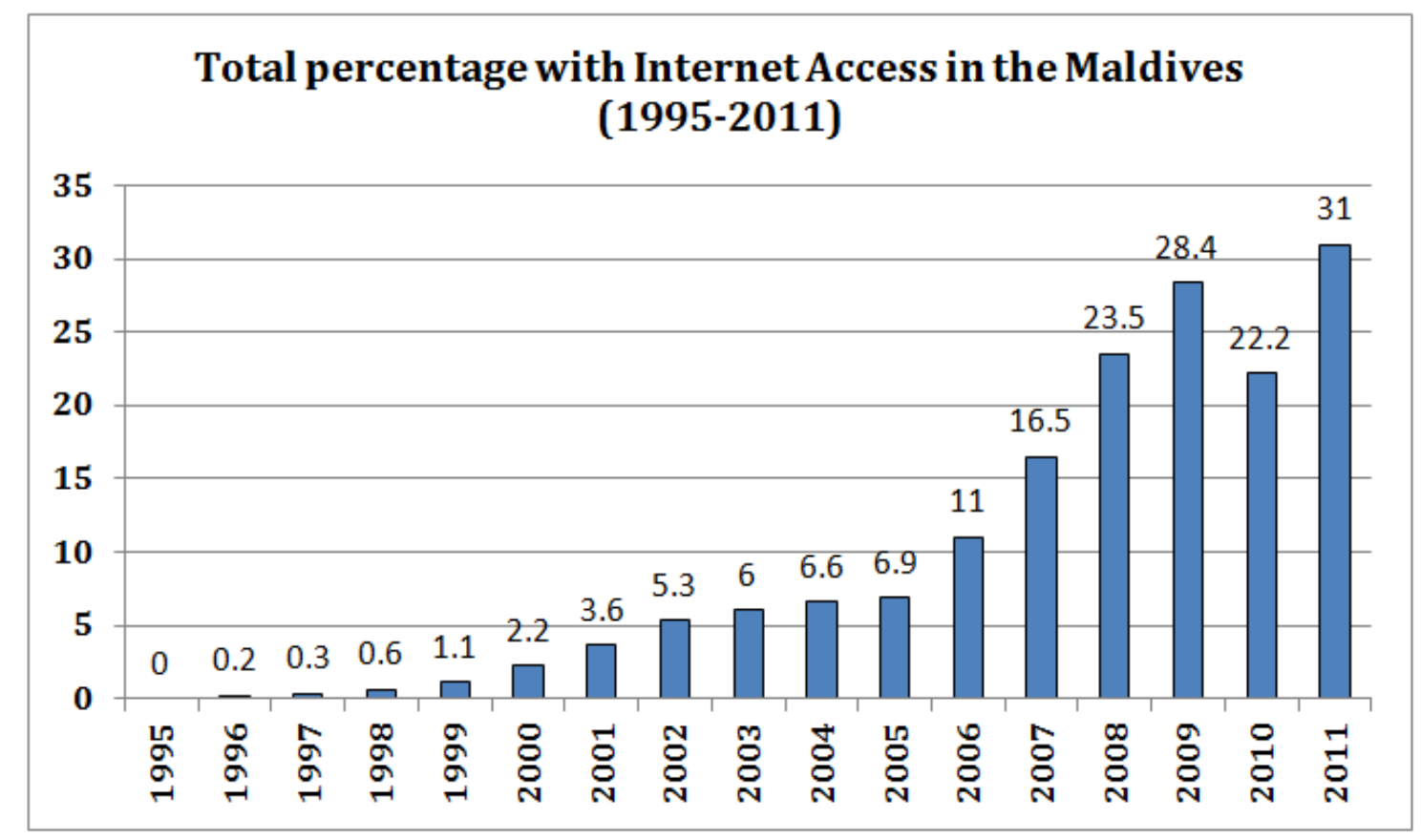

Figure 14: Internet users with 100 per inhabitants

Source: $\quad$ CAM, 2013; ITU, 2010

Despite the promising Internet penetration rate in the Maldives, Adam and Urquhart (2007) expressed concern that there is also a general lack of Internet affordability and accessibility because only two Internet Service Providers (ISPs) exist in the Maldives. Chan and Welebir (2003) noted that, because the Internet has 24 hour accessibility, people who do not attend conventional universities can obtain education at a distance more easily and study at their own pace.

\subsubsection{ICT Projects in the Maldives}

E-Government: The e-Government network (i.e. hardware and software) set up by the National Centre for Information Technology is a potential platform that can 
be used for ICT-based teaching and learning in the higher education sector. The eGovernment is not yet fully completed but it is now in the final stage.

Teacher Resource Centres (TRCs): The UNICEF-funded project of Teacher Resource Centres (TRCs) is a major network, which provides Internet connections in the atolls for students. TRCs are equipped with modern technology such as the "smart board", which is a replacement for the traditional white board used in schools (InfoDev, 2010a; InfoDev, 2010b). In the Maldives, higher education institutions lack the skills to fully utilise technology in curriculum implementation, therefore the traditional chalk and duster approach still dominates in higher education pedagogy. Teacher Resource Centres have given island students the opportunity to access quality education (UNICEF, 2010). The UNICEF representative for the Maldives Mr. Ken Maskall, said, "It's down to basics. Transport is costly, making it expensive and often dangerous for children to travel between islands to get better education and for teachers to update their skills" (UNICEF, 2010, p.1).

Teacher Resource Centres have helped island students and teachers to increase ICT competency skill levels, foster quality education, increase know-how and update their ICT knowledge. The Maldives' former Minister of Education Zahiya Zareer said "One of the main constraints of achieving our goal of quality education has been the remoteness of individual communities" (UNICEF, 2010, p.1). She also said: "the establishment of TRCs and the provision of broadband Internet with access to virtual learning environments is a giant leap towards achieving quality education" (UNICEF, 2010, p.1).

Furthermore, the UNICEF Representative in the Maldives, Ken Maskall also commented: "All children should have access to the same opportunities, regardless of where they live" (UNICEF, 2010, p.1). He also said: "A good quality education will help Maldivian children to make more valuable contributions to their communities" (UNICEF, 2010, p.1). 
Given the relatively high penetration of the Internet, the availability of WiFi is a priority enabling people to connect their own devices, rather than providing devices in a resource centre.

Maldives High-speed network rollout programme: A new submarine cable project in the Maldives will further increase the Internet connectivity to the islands. The project will be completed in 2013 by Dhiraagu which plays a major role in providing telecommunication services to the Maldives. The Maldives highspeed network rollout programme project allows broadband access from the islands at an affordable price. The Internet access will be through wired and wireless connections. The completion of this project will enable every island of the Maldives to have high-speed broadband. Even if there are only minimal Internet connections, the proposed solution will work for the remote islands.

Multi-Purpose Community Tele-Centres (MCTC): The existing MCTC and kiosks are potential tools which can be used in providing access to the Internet for higher education students and teachers. As MCTC are a new concept, they can be regarded as a resource centre for students and teachers. Multi-Purpose Community Tele-Centres are available in most of the highly populated islands. The initiative from The National Centre for Information Technology to establish the MCTC's in every inhabited island will help provide access to higher education, bridge the digital gap, and empower the islanders to take the opportunities available through ICT connectivity.

\subsubsection{ICT infrastructures}

Computers were introduced to the Maldives in the 1980s when the first minicomputer was imported (MCST, 2005a). The machine (i.e. model DG S120) was brought with assistance from the UNDP by the former Ministry of Planning and Development (Ibrahim, 2006).

The Strategic Action Plan (2009-2013) focuses on strengthening the ICT industry and extending the technology-based education in the Maldives. To that extent, it 
articulates policies and plans required to expand the ICT infrastructure and ensure affordability of ICT services for all citizens (InfovDev, 2010a).

The growth in the use of mobile phones is noteworthy in the Maldives with its advanced telecommunication systems. According to the UNCTAD (2009) report, Montenegro saw the greatest increase in mobile penetration, followed by Qatar, Bahrain and the Maldives. Table 8 shows the Maldives in $10^{\text {th }}$ place amongst the twenty most dynamic economies in terms of mobile penetrations, some of which are SIDS:

\begin{tabular}{|c|l|c|}
\hline No & \multicolumn{1}{|c|}{ Country } & $\begin{array}{c}\text { Mobile Penetration } \\
\text { devices per 100 } \\
\text { inhabitants }\end{array}$ \\
\hline 1 & Montenegro & 235 \\
\hline 2 & Qatar & 203 \\
\hline 3 & Bahrain & 190 \\
\hline 4 & United Arab Emirates & 178 \\
\hline 5 & Macao, China & 169 \\
\hline 6 & Lithuania & 150 \\
\hline 7 & Latvia & 146 \\
\hline 8 & Saint Kitts and Nevis & 143 \\
\hline 9 & Bulgaria & 142 \\
\hline 10 & Maldives & 138 \\
\hline 11 & Serbia & 136 \\
\hline 12 & Antigua and Barbuda & 135 \\
\hline 13 & Russian Federation & 132 \\
\hline 14 & Dominica & 124 \\
\hline 15 & Romania & 123 \\
\hline 16 & Oman & 121 \\
\hline 17 & Ukraine & 120 \\
\hline 18 & Argentina & 117 \\
\hline 19 & Trinidad and Tobago & 116 \\
\hline 20 & Uruguay & 105 \\
\hline & & \\
\hline
\end{tabular}

Table 8: The twenty most dynamic economies in terms of mobile penetration (2003-2008) Source: UNCTAD, 2009

According to the Asian Development Bank - ADB (2010) report, the Maldives was also among the ten most dynamic economies in the Asia and Pacific region where cellular phone subscriptions out-number people.

The Maldives' leading telecom service provider, Wataniya, launched an m-Learning service which is a distinct method that allows any person, regardless of location, to pursue education by utilising mobile devices (Wataniya, 2012). Speaking at the 
launch, the former Vice President of the Maldives, Dr. Mohamed Waheed Hassan, commended Wataniya's commitment to the improvement of education in the Maldives. Wataniya (2012) emphasised the importance of students no longer needing to travel to schools and colleges but only requiring a laptop and Wataniya Broadband connection.

The Maldives has invested heavily in the development of telecommunications and is regarded as having one of the best telecommunication infrastructures in the South Asia region (Asian Development Bank - ADB, 2010; ITU, 2009; ITU, 2008; TAM, 2011; UNESCAP, 2008). According to MTC (2006), the telecommunication policy of the Maldives:

Telecommunication can play a vital role in linking our dispersed communities and reduce the impact of the geographical isolation and physical separation that exists between island communities of Maldives. Telecommunications will also play a major role in achieving the economic and social developmental objectives envisaged in the Vision 2020 of the Maldives" (p.1).

Use of existing ICT resources (i.e. Internet technologies, networks, mobile technologies and computers) will allow teaching and learning to be carried out from remote islands. The teachers will have more teaching opportunities through the use of existing ICTs such as smart boards and multimedia technologies available in the TRCs at atoll level (InfoDev, 2010b).

\subsubsection{The Maldives compared with regional countries with regard to ICT}

Table 9 shows the ranking order statistics and comparison of countries in the SAARC region. The Maldives is placed in number one spot with regard to Internet users; mobile coverage; personal computer rate per 100 people; mobile coverage and TV coverage (ITU, 2010; Asian Development Bank - ADB, 2010): 


\begin{tabular}{|c|l|c|c|c|c|c|}
\hline No & Country & $\begin{array}{c}\text { Internet } \\
\text { Users }\end{array}$ & $\begin{array}{c}\text { Internet } \\
\text { penetration } \\
\text { rate \% }\end{array}$ & $\begin{array}{c}\text { Personal } \\
\text { Computer } \\
\text { (per 100) }\end{array}$ & $\begin{array}{c}\text { Mobile } \\
\text { coverage \% } \\
\text { as per } \\
\text { population }\end{array}$ & $\begin{array}{c}\text { TV with } \\
\text { household } \\
\text { \% as per } \\
\text { population }\end{array}$ \\
\hline 1 & Maldives & 120,000 & 28.9 & 20.08 & 103 & 92 \\
\hline 2 & Pakistan & $20,350,000$ & 11.5 & 0.46 & 90 & 46.5 \\
\hline 3 & Sri Lanka & $1,776,200$ & 8.3 & 3.54 & 90 & 31.6 \\
\hline 4 & Bhutan & 50,000 & 7.1 & 0.6 & 21 & 57.7 \\
\hline 5 & India & $81,000,000$ & 6.9 & 3.17 & 61 & 32 \\
\hline 6 & Afghanistan & $1,000,000$ & 3.4 & 0.32 & 72 & 6.2 \\
\hline 7 & Nepal & 625,800 & 2.2 & 0.49 & 10 & 13.1 \\
\hline 8 & Bangladesh & 995,560 & 0.6 & 2.42 & 90 & 22.9 \\
\hline
\end{tabular}

Table 9: $\quad$ ICT in South Asian Countries (SAARC countries)

Source: Internet World Stats, 2013

The Maldives is enjoying one of the highest mobile phone ownership rates in the region (ITU, 2010). Figure 15 shows a representation of the mobile technology coverage in the SAARC region:

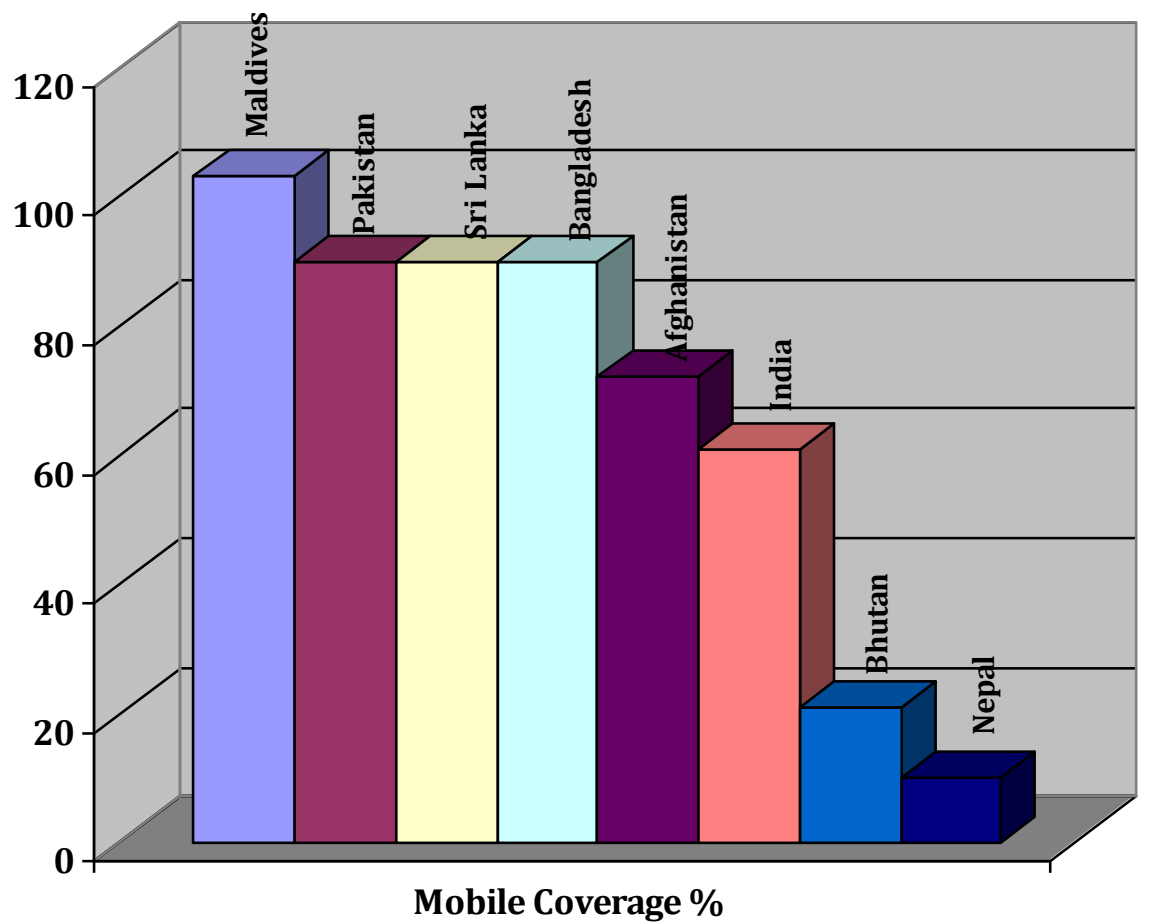

Figure 15: Mobile coverage in South Asian Countries (SAARC countries) Source: Internet World Stats, 2013

The access to IT is likely to change even more in the near future through the Mobile Communications Technology (MCT) to reduce the digital divide and empower the islanders to take the opportunities available through ICT connectivity (InfoDev, 
2010a). The use of MCT will enable students and teachers to use 3G and mobile technologies in the learning process. In addition, according to the International Telecommunication Union, the Maldives were among the top ten economies that gained most in value on the ICT access sub index between 2002 and 2007 (ITU, 2010).

The National Development Plan also stated as Policy 1 of its information technology chapter to "enable all students to use computers for communication, information dissemination and research" (Department of National Planning, 2011, p.91).

\subsubsection{SIDS comparison in terms of geography and ICT}

Table 10 illustrates the comparison of ICTs within the SIDS. The table illustrates the geographical area, total population, internet users, internet penetration rate and mobile cellular coverage of SIDS.

In terms of Internet penetration, the Maldives is rated as 29 out of 52 SIDS. The highest three highest Internet penetration rates are listed as St. Lucia, Niue, and Antigua and Barbuda. Internet penetration is high among SIDS.

In terms of mobile cellular penetration rates, Trinidad and Tobago is the highest with Antigua and Barbuda second highest and Suriname as third highest. The mobile cellular penetration rate is also extremely high in some SIDS. The Maldives has a mobile cellular percentage rate of $153 \%$ (i.e. on average one person has 1.5 mobile cellular phones). 


\begin{tabular}{|c|c|c|c|c|c|c|c|}
\hline No & Country & $\begin{array}{c}\text { Area (sq } \\
\mathbf{k m})\end{array}$ & $\begin{array}{c}\text { Total } \\
\text { Population }\end{array}$ & $\begin{array}{l}\text { Internet } \\
\text { Users }\end{array}$ & $\begin{array}{c}\text { Internet } \\
\text { penetration } \\
\text { rate } \%\end{array}$ & $\begin{array}{l}\text { Mobile } \\
\text { Cellular }\end{array}$ & $\begin{array}{c}\text { Mobile } \\
\text { Cellular } \\
\% \\
\end{array}$ \\
\hline 1 & St. Lucia & 616 & 161,557 & 142,900 & 88.5 & 215,500 & 133 \\
\hline 2 & Niue & 260 & 1,311 & 1,100 & 83.9 & - & - \\
\hline 3 & Antigua and Barbuda & 442.6 & 87,884 & 70,968 & 80.8 & 176,000 & 200 \\
\hline 4 & Singapore & 697 & $4,740,737$ & $3,658,400$ & 77.2 & $7,794,000$ & 164 \\
\hline 5 & $\begin{array}{l}\text { St. Vincent and the } \\
\text { Grenadines }\end{array}$ & 389 & 103,869 & 76,000 & 73.2 & 131,800 & 126 \\
\hline 6 & Barbados & 430 & 286,705 & 191,878 & 66.9 & 347,900 & 121 \\
\hline 7 & British Virgin Islands & 151 & 25,383 & 14,620 & 57.6 & 46,800 & 184 \\
\hline 8 & Bahrain & 760 & $1,214,705$ & 649,009 & 57.1 & $1,694,000$ & 139 \\
\hline 9 & Jamaica & 10,991 & $2,868,380$ & $1,581,100$ & 55.1 & $2,975,000$ & 103 \\
\hline 10 & Cook Islands & 236 & 11,124 & 6,000 & 53.9 & 7,800 & 70 \\
\hline 11 & Trinidad and Tobago & 5,128 & 222,505 & 650,611 & 53.2 & $1,825,000$ & 820 \\
\hline 12 & Bahamas & 13,880 & 313,312 & 158,700 & 50.7 & 298,800 & 95 \\
\hline 13 & Guam & 544 & 183,286 & 90,000 & 49.1 & 98,000 & 53 \\
\hline 14 & Aruba & - & 106,113 & 51,280 & 48.3 & - & - \\
\hline 15 & Dominican Republic & 48,670 & $9,956,648$ & $4,643,393$ & 46.6 & $8,770,000$ & 88 \\
\hline 16 & Anguilla (UK) & 91 & 15,094 & 6,940 & 46.0 & 26,000 & 172 \\
\hline 17 & French Polynesia & 4,167 & 132,674 & 294,935 & 45.0 & 222,800 & 167 \\
\hline 18 & St. Kitts and Nevis & 261 & 50,314 & 22,340 & 44.4 & 84,600 & 168 \\
\hline 19 & Dominica & 751 & 72,969 & 32,151 & 44.1 & 111,000 & 152 \\
\hline 20 & Puerto Rico & 13,790 & $3,989,133$ & $1,698,301$ & 42.6 & $3,108,000$ & 80 \\
\hline 21 & Tuvalu & 26 & 10,544 & 4,300 & 40.8 & 2,100 & 20 \\
\hline 22 & Seychelles & 455 & 89,188 & 33,900 & 38.0 & 126,600 & 141 \\
\hline 23 & Netherlands Antilles & - & 199,800 & 92,500 & 37.2 & - & - \\
\hline 24 & $\begin{array}{l}\text { Commonwealth of Northern } \\
\text { Marianas }\end{array}$ & 464 & 46,050 & 15,980 & 34.7 & - & - \\
\hline 25 & New Caledonia & 18,575 & 256,275 & 87,420 & 34.1 & 227,300 & 87 \\
\hline 26 & Suriname & 163,820 & 491,989 & 44,000 & 33.1 & 947,000 & 192 \\
\hline 27 & Grenada & 344 & 108,419 & 34,961 & 32.2 & 121,900 & 112 \\
\hline 28 & Guyana & 214,969 & 744,768 & 220,000 & 29.5 & 528,800 & 71 \\
\hline 29 & Maldives & 298 & 350,759 & 114,100 & 28.9 & 540,004 & 153 \\
\hline 30 & Cape Verde & 4,033 & 516,100 & 148,800 & 28.8 & 396,400 & 77 \\
\hline 31 & Palau & 459 & 20,956 & 5,980 & 28.5 & 15,400 & 73 \\
\hline 32 & US Virgin Islands & 1,910 & 109,666 & 30,000 & 27.4 & 80,300 & 73 \\
\hline 33 & Mauritius & 2,040 & $1,303,717$ & 323,494 & 24.8 & $1,294,000$ & 99 \\
\hline 34 & Montserrat & 102 & 5,140 & 1,200 & 23.3 & 4,200 & 82 \\
\hline 35 & $\begin{array}{l}\text { Federated States of } \\
\text { Micronesia }\end{array}$ & 702 & 106,836 & 22,213 & 20.8 & 27,500 & 26 \\
\hline 36 & Belize & 22,966 & 321,115 & 63,580 & 19.8 & 222,000 & 69 \\
\hline 37 & Fiji & 18,274 & 883,125 & 162,880 & 18.4 & 727,000 & 82 \\
\hline 38 & Sao Tome and Principe & 964 & 179,506 & 31,012 & 17.3 & 115,000 & 64 \\
\hline 39 & Cuba & 110,860 & $11,087,330$ & $1,702,206$ & 15.4 & $1,315,000$ & 12 \\
\hline 40 & Tonga & 747 & 105,916 & 12,487 & 11.8 & 55,000 & 5 \\
\hline 41 & Haiti & 27,750 & $9,719,932$ & $1,000,000$ & 10.3 & $4,200,000$ & 43 \\
\hline 42 & Marshall Islands & 181 & 67,182 & 6,540 & 9.7 & 3,800 & 6 \\
\hline 43 & Kiribati & 811 & 100,743 & 8,959 & 8.9 & 13,800 & 14 \\
\hline 44 & Vanuatu & 12,189 & 224,564 & 19,172 & 8.5 & 137,000 & 61 \\
\hline 45 & Samoa & 2,831 & 193,161 & 12,813 & 6.6 & 167,400 & 87 \\
\hline 46 & Comoros & 2,235 & 794,683 & 34,472 & 4.7 & 216,400 & 27 \\
\hline 47 & Solomon Islands & 28,986 & 571,890 & 26,907 & 4.7 & 274,900 & 48 \\
\hline 48 & American Samoa & 199 & 67,242 & 3,040 & 4.5 & - & - \\
\hline 49 & Nauru & 21 & 9,322 & 340 & 3.6 & 6,700 & 72 \\
\hline 50 & Guinea-Bissau & 36,125 & $1,596,677$ & 37,123 & 2.3 & 869,100 & 54 \\
\hline 51 & Papua New Guinea & 462,840 & $6,187,591$ & 125,000 & 2.0 & $2,400,000$ & 39 \\
\hline 52 & Timor-Leste & 14,874 & $1,177,834$ & 2,361 & 0.2 & 614,200 & 52 \\
\hline
\end{tabular}

Table 10: SIDS geographical context with ICT

Sources: Internet World Stats, 2013; Central Intelligence Agency - CIA (2012); CAM, 2013 


\subsection{Chapter Summary}

This chapter gave a literature overview about the Maldives. The chapter started by introducing the Maldives, its geography, population and economy. The chapter shed light on the culture, primary, secondary and higher education contexts and ICT infrastructure in the Maldives. The chapter also highlighted the ICT infrastructure in the Maldives and the main ICT initiatives.

This chapter summed up the particular issues in the Maldives that are related to this research. The geographical distribution is the major issue, which limits the higher education services and low enrolment in the islands. The chapter outlined the availability of ICT resources within the Maldives, which is a potential advantage where we can utilise in higher education. The next chapter focuses on the research procedure and what actually happened during the data collection process. 


\section{CHAPTER 5}

\section{RESEARCH PROCEDURE}

\subsection{Introduction}

This chapter focuses on the actual research process. Based on the number of students enrolled and the quality and reputation of the Maldives' higher education institutes, seven research sites were considered. Three sites were selected for data collection. These three research sites were reputable tertiary institutions in the Maldivian higher education sector.

The chapter describes how the data was collected and analysed. The data analysis section of this chapter explains the translating, transcribing, and coding processes, theme generation procedures and conceptualisation of major themes. The chapter concludes by outlining the data collection experience and roles played by the researcher.

\subsection{Research Sites}

\subsubsection{Short listed Data Collection at Higher Education Institutes}

The research site selection was carried out by the researcher using a selected list of higher education institutes based on the researcher's experience and the reputation of the institutes. Key stakeholders were selected for interviewing from amongst the higher education and information technology sectors, Government organisations, private organisations, and ICT and education experts in the Maldives. The seven institutions were:

1. Maldives College of Higher Education

2. Villa College

3. Cyryx College

4. Modern Academy for Professional Studies (MAPS) College

5. Clique College

6. Mandhu College

7. Focus Education Centre 
The researcher selected a total of three institutions (i.e. one large public higher education institute and two private higher institutes). The selected three tertiary institutes were the Maldives College of Higher Education, Villa College and Clique College. The large public higher education institute was the most reputable among the seven. The other two private higher education institutes (Villa College and Clique College) had good enrolments with higher reputations with regard to teaching and learning.

The researcher gained access to the three tertiary institutes by presenting the research purpose, explaining the importance of the research and the motivation for doing this kind of research in the context of the Maldives.

\subsubsection{Access to research sites}

Access to the research sites was easy since the researcher was familiar with the tertiary institutes (Maldives College of Higher Education, Villa College and Clique College) and had good rapport with the members of staff. Before the researcher came to New Zealand for doctoral study, he had actively participated in lecturing at two of these institutes (The Maldives College of Higher Education and Villa College). This saved time in gaining acceptance and familiarity with the research sites and respondents.

The researcher enclosed a request for participation letter to the selected three higher institutes (see Appendix 6) to obtain a prime contact person. The prime contact person was senior person who could able to identify the appropriate people to contact (Voss et al., 2002). The three institutions assigned senior staff members as prime contacts, which enabled the researcher to liaise and coordinate easily during the data collection process.

The higher education institutes all welcomed the researcher. This was an advantage because the teachers, students and academic management were willing to share their experience with the researcher and had good working relationships. 
The researcher obtained formal approval for access to the designated tertiary institutes. The sample consent forms for higher education institutes are attached (see Appendix 7). The higher education institutes, through written documentation, granted formal approval. Once approval had been granted to undertake the research, direct access to teachers, students, academic management and key stakeholders was possible.

Accessing the research sites during official working hours was avoided since it might disrupt the teachers, students and academic management. Creswell (2003) stated, "... researchers need to respect research sites so that the sites are left undisturbed after the research study" (p.65). The researcher ensured that no disturbance was caused during the course of the data collection.

There were separate formal meetings with the heads of the three institutes who promised full cooperation during the course of the data collection process. The heads of the tertiary institutes requested their line academic managers, teachers and students to also help the researcher.

After the formal introduction to the heads of the tertiary institutes, the researcher was provided with a list of prime contacts in the institutes. The prime contacts came up with a list of potential interviewees from each tertiary institute and had access to students and teachers for the focus groups.

The researcher had an opportunity to have informal meetings with interested staff to brief them about the research. These meetings were helpful to convey the importance of this research, convince the academic management to participate in it, and helped in selecting people for the interviewing and formation of focus groups.

\subsubsection{Maldives College of Higher Education}

The Maldives College of Higher Education (MCHE) is the largest public tertiary institute in the Maldives. The researcher worked there as a part-time lecturer for more than ten years. Operating under the aegis of the Ministry of Education, MCHE 
is the only public degree-granting institution in the Maldives. The institute offers a range of bachelor's degrees, Master's Degrees and doctoral degree courses. MCHE was selected as a research site since it is the largest public tertiary institute in the Maldives.

The Rector of MCHE expressed his enthusiasm about the usefulness of such a study at a very important stage where the Maldives higher education system required a change. He also promised his full support for the researcher and requested the Deans of the faculties to provide support.

\subsubsection{Villa College}

Villa College is privately owned by a multi-millionaire and Member of Parliament (Hon. Qasim Ibrahim), a philanthropist who has helped the Maldivians by providing services such as medical services, education and social services. Villa College prides itself on offering quality and internationally recognised tertiary education opportunities to many Maldivians, at an affordable price (Villa College, 2011).

Villa College works in collaboration with different universities and professional bodies. The College offers a variety of courses in business, information technology, education, tourism, hospitality, marine studies and Islamic shariah (Villa College, 2013). Villa College is the largest private institution and the first private institute to offer degree level courses to Maldivians (Villa College, 2013). Villa College was chosen as the second research site due to its higher reputation in the Maldives.

Access to Villa College was easy. The senior management welcomed the researcher and were very impressed with the research. Villa College senior management gave permission to interact with staff, teachers and students. The rector of Villa College emphasised the importance of such academic research. He promised to provide his full support for the researcher and instructed all senior members of the faculties to help in obtaining the required information for the researcher. Villa College senior management also gave permission to interact with staff, teachers and students 


\subsubsection{Other Institutes}

Clique College is a private tertiary institution owned by a group of local businessmen. Clique College offers quality and internationally recognised tertiary education opportunities to many Maldivians (Clique College, 2013). It works in collaboration with different universities and professional bodies. Clique College offers a variety of customised degree programmes in information technology and business (Clique College, 2013). Clique College is an emerging tertiary institute offering a variety of courses. Clique College was chosen since it offers a variety of courses to the public, and is one of the most prestigious institutes among Maldivians. Gaining access to Clique College was not difficult. The management welcomed the researcher and were impressed with the research. There was also one interviewee from Mandhu College, where the participant was working parttime.

\subsubsection{Selecting Research Sites}

Upon receiving formal confirmation from the prime contact, the researcher briefed the research participants about the procedures for collecting data from their institutes. This gave the prime contact person sufficient time to obtain relevant documentation and information for the study before the data collection process commenced. In addition, it also provided extra information, which was helpful for the researcher. Figure 16 shows the sites selected.
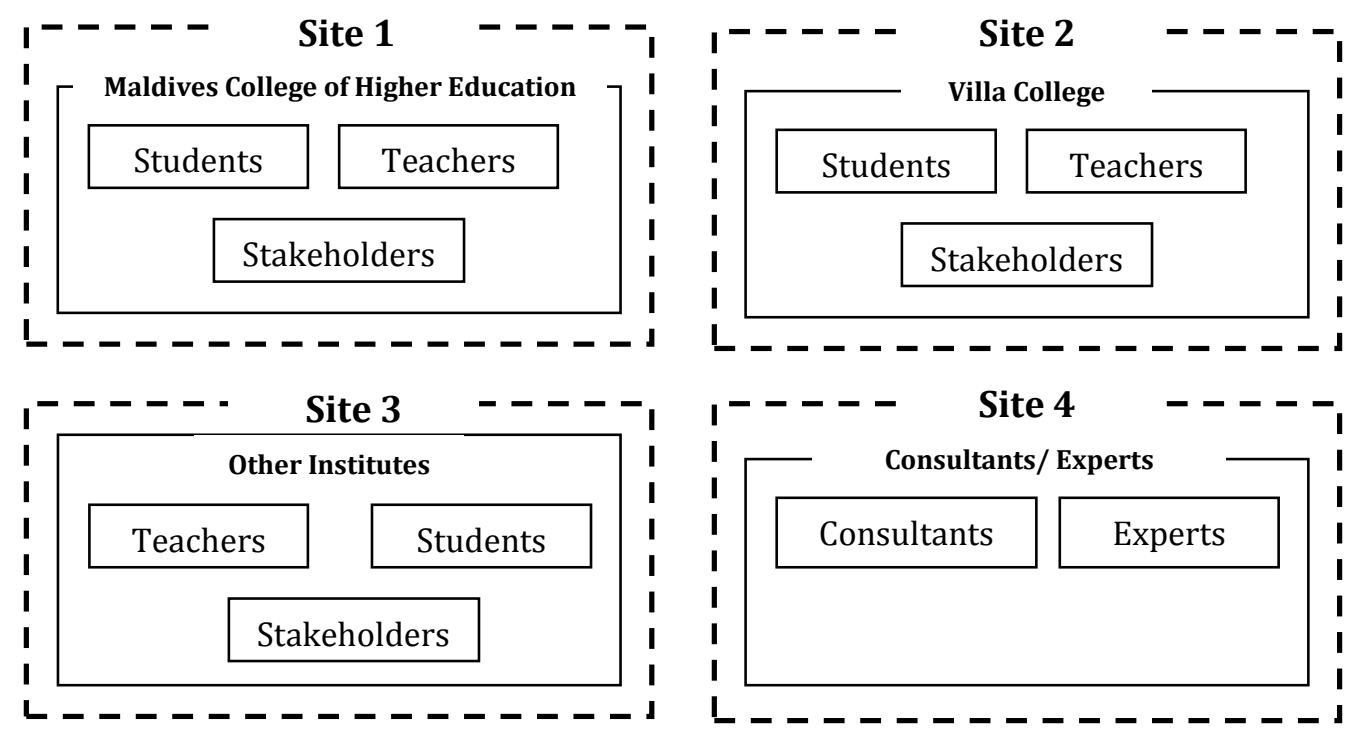

Figure 16: Site Selection 
Site 1: The research participants included students, teachers and stakeholders from The Maldives College of Higher Education.

Site 2: The research participants included students, teachers and stakeholders from Villa College.

Site 3: The research participants included students, teachers and stakeholders from the other institutes (i.e. Clique College and Mandhu College).

Site 4: $\quad$ The research participants included consultants and experts from education and information technology sectors.

\subsubsection{Access to interviewees}

The researcher began with a list of 45 interviewees to contact. The contact numbers of the interviewees were provided by the institutes and the researcher had no problem gaining access. A total of 32 interviews were successfully reached by contacting the interviewees, all of whom consented to participation. The remaining 13 initial contacts were not able to provide interviews for this study due to unavailability and busy work schedules.

Here is the list of organisations that provided interviewees for this research.

- Education Development Centre

- Elections Commission

- Civil Service Commission

- Clique College

- Department of Higher Education and Training

- Info Tech Maldives Private Limited

- Maldives College of Higher Education

- Mandhu College

- Ministry of Education

- Ministry of Higher Education, Human Resources and Social Security

- National Centre for Information Technology

- Office of the Audit 
- Teacher Resource Centre at Upper South Province

- Villa College

\section{Role of Interviewees}

\begin{tabular}{|l|c|}
\hline \multicolumn{1}{|c|}{ Labels } & No of respondents \\
\hline Coordinator & 1 \\
\hline Education Administrator & 1 \\
\hline Education Consultant & 1 \\
\hline Executive in higher education & 8 \\
\hline Government Department & 3 \\
\hline Executive & 2 \\
\hline Government Department Leader & 1 \\
\hline Information Technology Executive & 3 \\
\hline Information Technology Expert & 8 \\
\hline Lecturer & 4 \\
\hline Student & \\
\hline
\end{tabular}

Table 11: Role of Interviewees with total numbers

Table 11 shows a summary of respondents from the selected research sites. There were a total of 32 respondents including education administrators, education consultants, executives in higher education, government department executives, government department leaders, information technology executives, information technology experts, lecturers and students.

The researcher met with interviewees before the actual interviews in an informal session. These informal chats with the respondents provided an opportunity to go through the interview questions, which the researcher had developed. This was a good exercise since the respondents were able to understand the nature of the research and had sufficient time to prepare beforehand. Research of this nature is scarce in the Maldives and some interviewees required more general information.

The researcher provided an information sheet to all interview participants (see Appendix 8). Before each interview, the researcher ensured that research 
participants had signed the interview consent form. A sample consent form is attached (see Appendix 9).

\subsubsection{Focus group participants}

There were four focus group discussions. The size of each focus group was 7-9 participants. The first two groups consisted of final year Bachelor of Information Technology students. These students were selected from the Maldives College of Higher Education. The third group consisted of final year Bachelor of Business Administration students from Villa College. The final group consisted of teachers, IT experts and education experts. There were no participants from Clique College in the focus groups.

\begin{tabular}{|c|l|l|c|}
\hline No & \multicolumn{1}{|c|}{ Group name } & \multicolumn{1}{c|}{ Label } & $\begin{array}{c}\text { No of } \\
\text { participants }\end{array}$ \\
\hline 1 & Information Technology - Focus Group 1 & Student - Focus Group & 8 \\
\hline 2 & Information Technology - Focus Group 2 & Student - Focus Group & 7 \\
\hline 3 & Business - Focus Group 3 & Student - Focus Group & 10 \\
\hline 4 & $\begin{array}{l}\text { Student, Teachers, Consultants, Student- } \\
\text { teachers and Experts - Focus Group 4 }\end{array}$ & $\begin{array}{l}\text { Student, Teachers, } \\
\text { Consultants, Student- } \\
\text { teachers and Experts }\end{array}$ & 8 \\
\hline
\end{tabular}

Table 12: Summary of focus group members

Table 12 shows a summary of participants in the four focus groups, together with group names, label and number of participants in each focus group.

\section{Group 1:}

Group 1 consisted of eight participants. The Information Technology group consisted of one man and seven women. These were students from the final year of the Bachelor of Information Technology programme at the Maldives College of Higher Education. Generally, this group were experienced in information technology concepts.

\section{Group 2:}

Group 2 consisted of seven participants. The Information Technology group consists of five men and two women. These were also students from the final year 
of the Bachelor of Information Technology programme at Maldives College of Higher Education. Generally, this group were also well experienced in information technology.

\section{Group 3:}

Group 3 consisted of ten participants. The Business Studies group of students consisted of four men and six women. These were students from the final year of the Bachelor of Business programme at Villa College. Generally, this group were less experienced in Information Technology concepts.

\section{Group 4:}

Group 4 consisted of eight participants. This group of students consisted of eight men only. These were a student, teachers, consultants, student-teachers and Experts. Generally, this group had good knowledge in their area of specialisation. This group was a mixture of people from different backgrounds with a rapport was built amongst participants.

All the participants in the focus groups were willing to participate. The researcher did not have any informal meeting with the focus group members but provided a list of questions before the focus group discussion commenced to ensure that participants understood the research context.

The participants in the focus group discussions were active. The focus group discussions offered a consultative opportunity to actively engage in providing information about participants' beliefs about ICT use and their perception of using ICT in the higher education.

Some members were also enthusiastic to provide information because they had never participated in a focus group session before. Nevertheless, the level of expertise was good since most of the participants were actively engaged in the higher education sector. The average focus groups sessions were about 90 minutes long, but some sessions lasted for more than 120 minutes. An information sheet for focus group discussion (see Appendix 10) was distributed to all focus group 
participants. Before commencement, the researcher ensured all participants had signed the focus group consent form. A sample consent form for focus groups is attached (see Appendix 11).

\subsubsection{Qualitative Survey participants}

The qualitative survey was a good method for capturing other data that was required for this research. A total of 350 survey forms were distributed to teachers and students through paper-based and e-mail delivery. The researcher received 100 forms that were completed and correctly returned. This return rate of just under $29 \%$ was reasonable and reflected the fact that both students and teachers had a keen interest in contributing to the research. There was a limited response rate through e-mails.

\subsubsection{Analysis of Qualitative Survey}

The qualitative survey, which involved students and teachers, was analysed using Microsoft ${ }^{\circledR}$ Excel. The researcher created a Microsoft ${ }^{\circledR}$ Excel worksheet and entered the data. There were 92 responses from students and 8 responses from teachers. There were a total of 41 male and 59 female respondents.

The age groups of the participants were also analysed. It was found that most participants (75 participants) fell into the age group of 16-25 years. This was followed by 26-35 years (24 participants) and 36-45 years ( 1 participant). None of the participants was older than 45 .

\subsubsection{Documents Reviewed}

Document reviewing was an important concept to get an overall idea of the research context. The document reviewing process had commenced before the primary research. As discussed in Chapter 4, the reviewed documents gave useful insight into the research context. Obtaining existing records from the Maldivian organisations was important. These records included higher education and ICT archival material, unpublished documents, archival material, e-Government initiative reports, and other reports. 
Typical documents reviewed were records from the higher education sector, ICT and unpublished documents in higher education institutes. Other specific documents included enrolment details, strategic plans, teaching and learning plan, operational priorities and courses offered.

\subsubsection{Informed Consent}

Obtaining informed consent from participants is an important step in conducting research because it recognises the autonomy of research participants (Johnson \& Christensen, 2008). Informed consent means that participants understand what they are agreeing to, accept what is being asked and are comfortable in providing the information (Pickard, 2007). Johnson and Christensen (2008) defined informed consent as “... agreeing to participate in a study after being informed of its purpose, procedures, risks, benefits, alternative procedures, and limits of confidentiality" (p.109).

The researcher develops an informed consent form for participants to sign before the research commences (Creswell, 2009; Neuman, 2011). Consent is an ethical tenet of voluntary participation, which cannot force anyone to participate in the research (Neuman, 2011). Seidman (1998) remarked that consent of the participant is important and a signature is required.

The researcher translated the consent form for those participants who had difficulty in understanding English. All the focus groups were conducted in the Dhivehi language since some participants were not fluent in speaking and understanding English. Relevant information was provided about the confidentiality of the data collected through these discussions and the promise that the data collected would not be used for any other purpose.

The interviews and focus group sessions were audio-recorded and the participants were fully aware of and understood the terms and conditions. For the interviews, the researcher read out the consent form and explained that the participant was voluntary. The interviewees were informed that the interviews would be audiorecorded and the participants fully understood the terms and conditions. 
The researcher provided information about protecting the confidentiality of participants' identities and data and promised that the data collected would not be used for any other purpose except for this research. On average the duration of the interviews was about 45-60 minutes per participant. Special attention was given to the issues of trust, which meant the researcher had a chance to establish good rapport with the participants in order to maintain the reliability and credibility of the research.

For the focus group discussions, the consent form, which explained that the focus group participation was expected from the participants without influence from anyone, was read out. The researcher pointed out that the participants were free to withdraw from participating in the research at any time without penalty. The nature of the research was explained to ensure that everybody understood the process. It is important for respondents to understand the elements of consent, in order to expect what to anticipate in the research (Creswell, 2003).

\subsubsection{Data Collection process}

The interview data was obtained from participants from the higher education institutes, information technology sector, higher education sector and experts from information technology and education sector. The focus groups' data was collected from four focus group discussions among students and teachers.

It is important to note that there were some qualified educational and information technology experts available within the Maldives who were able to provide substantial input into this research. The researcher met with senior government officials from the Ministry of Education and information technology sector.

The languages chosen for this research were both the local Maldivian language (Dhivehi) and English. The researcher advised the participants to choose in which language they were more comfortable to speak and communicate well. Any written documents in Dhivehi were translated into English by the researcher. There were no risks associated in speaking Dhivehi, which also gave participants the advantage of expressing their ideas fluently. 
The translations of recorded materials (Dhivehi interviews and focus groups discussions) were verified by an independent consultant. The translated and transcribed materials were checked by the consultant for translation errors.

\subsection{Data Analysis}

As this was a qualitative study, the raw data in the form of words from the interview and focus group sessions were not meaningful in themselves. Figure 17 shows the four main steps involved in the data analysis process: a) translating and transcribing (Punch, 2005); b) identification of themes (Neuman, 2011); c) coding data (Neuman, 2011; Punch, 2005) and d) conceptualisation of themes (Neuman, 2011).

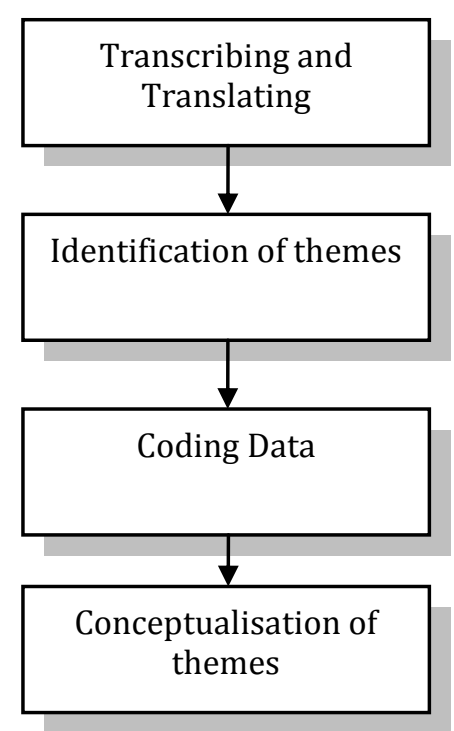

Figure 17: Data analysis process (adapted from Punch, 2005 and Neuman, 2011)

\subsubsection{Translating and transcribing}

The digital audio-recorded versions of the interviews and focus group sessions were displayed by playing the recorded version through a multimedia player, which the researcher had control to start and stop by pausing. The interview recordings were transcribed verbatim.

There were a total of 32 interviews and four focus group sessions to transcribe. Out of the 32 interviews, 18 were in the Dhivehi Language, which the researcher had to translate and transcribe. The remaining 14 interviews were conducted in 
English. All four focus groups sessions were conducted in the Dhivehi Language and the comments were also translated and transcribed.

After transcribing all the interviews and focus groups sessions, the transcripts were checked against the recordings for a second time. Softcopies of the interview transcripts were e-mailed to the respondents to check for credibility. Some respondents did not reply, which the researcher assumed meant their transcripts were correct. There were no issues raised by the research participants. The researcher felt that returning the interview transcripts to the participants enabled greater trustworthiness. A minimised version of a sample interview transcript is attached (see Appendix 12). A minimised version of one of the focus group discussion transcripts is also attached (see Appendix 13).

\subsubsection{Identification of themes}

The coded data was sorted into arrays according to the major categories, giving special attention to the research questions. All the chunks of data that had the same labels or closely related labels were categorised. Theme identification is one of the most basic tasks in qualitative research.

\subsubsection{Coding the data}

Qualitative analysis starts with coding which is a core feature (Creswell \& Clark, 2007; Denscombe, 1999; Neuman, 1997) which is crucial to effective case research (Voss et al., 2002). Dividing the text into small units and grouping and assigning labels to each (Creswell \& Clark, 2007; Neuman, 1997). These labels were derived from the data received from the interviews, focus group sessions, document reviewing and qualitative survey. Codes are tags or labels for assigning units of meaning (Neuman, 2011). Codes are especially useful tools for data reduction purposes (Denscombe, 1999; Neuman, 1997). .

The researcher used NViVo software to analyse the qualitative data. NViVo also allowed the researcher to link, annotate, and create relationships which eventually reshaped and reorganised the coding easily (Creswell \& Clark, 2007; Neuman, 
2011). The software has the capacity to organise codes in diagrammatic form (Creswell \& Clark, 2007; Neuman, 1997).

The researcher found that NViVo software was a useful tool to index words or segments of text / short phrase into identified themes. NViVo database was created for interviews and focus group discussion transcripts are saved in a file. The researcher went through the data looking for pieces of data that were applicable to answering the two research questions. Coding was carried out by reading the interview and focus group transcripts.

Due to interpretive nature of this research, it was important to find out the relationships among themes by comparing one or more code. The researcher was familiar with NViVo and had no trouble in manipulating the software. Sample NViVo tree nodes are attached (see Appendix 14).

\subsubsection{Conceptualisation of themes}

The conceptualisation of themes tied the data together and paved the way in answering the research questions. The researcher found that this was a critical step in understanding the relationships from the gathered data. Coded themes were juggled to create conceptual diagrams or mappings. The diagrams were drawn using Microsoft ${ }^{\circledR}$ Visio, which made it easy to create conceptual diagrams. The basic ideas for conceptualisation were to document the general idea inferred from specific instances of the data collected. A sample conceptualisation diagram is attached (see Appendix 15).

\subsection{Data Collection Experience}

The data collection depended on interaction between respondents (i.e. data) and the researcher, which pointed to interdependence between the data and researcher. Since the data was qualitative in nature, large amounts were collected from a limited number of respondents. The researcher had to depend on information from a few respondents in this research. 
In the data collection process, the researcher interviewed 32 people and undertook four focus group discussions. The interview participants mostly highlighted the crucial need to utilise ICT in the higher education sector. Some participants had a personal interest in contributing since they knew that nobody has undertaken research of this nature in the Maldives.

\subsection{Roles of the Researcher}

The researcher had worked as a lecturer at The Maldives College of Higher Education (MCHE), Villa College and Clique College before arriving in New Zealand for doctoral studies. Some of the researcher's other related activities included working as an Information Technology Officer for the United Nations International Children's Emergency Fund (UNICEF) for a total of four years and as an Information Technology staff member in The President's Office in the Maldives for ten years.

To undertake this research, it was vitally important to have good knowledge of the Maldives' higher education institutions, its people, problems and current as well as potential issues. Having previously established rapport and trust with many of the main actors in the higher education sector was an added advantage.

In interviews, the researcher avoided leading questions (i.e. questions that tend to elicit a particular response) that might influences respondents' answers. The interview questions were reviewed to ensure that no leading questions were included. The researcher was aware that a leading question might result in a leading answer, which might be untrue or mask the truth.

In the focus group sessions, the researcher assigned a skilled moderator to undertake the research to reduce direct involvement. The moderator had the skills to control the session to avoid dominant respondents who could influence other respondents by taking more time. 
In the qualitative survey, the researcher employed a similar approach such as avoiding biased questions. The qualitative survey questionnaire was made short and simple to ensure the participants could fill in the forms easily.

The researcher built a close relationship with the higher educational institutes; key stakeholders and ICT sector personnel in the Maldives to carry out this research. Throughout the research phase, the researcher ensured the study was well constructed to ensure reliability and credibility. The researcher participated in the focus group discussions as an observer to gain an empathic and in-depth understanding. A skilled moderator was employed to undertake the focus groups discussions to avoid considerations of bias.

Walsham (2006) explained that interpretive researchers need to gain access to relevant organisations for data collection. Gaining access to the research sites was not a problem for the researcher, since he already had assurances of co-operation from the higher education institutes contacted.

Walsham (1995a) explained that interpretive researchers are attempting the difficult task of accessing other people's interpretations and also have a view of their own role. Creswell (2003) emphasised that there also are other roles for researchers, such as addressing ethical issues that might arise (see Pickard, 2007).

Gaining entry to the research site was an important issue to consider. The first step was writing to or informing each potential contact from the organisation (Voss et al., 2002). Some key personnel in the higher education sector of the Maldives were contacted before the researcher arrived for data collection. This ensured a smooth data collection process with easy access to the higher education institutes.

\subsection{Chapter Summary}

This chapter highlighted how the data was collected by the researcher, how the research sites were selected and what happened during the research process, as well as describing the data collection sites, access to research participants and 
ethical considerations. The chapter documented how the actual data was analysed. The data analysis was explained in detailed, such as translating, transcribing, coding data and conceptualisation of major themes. The chapter concluded by explaining the data collection experience and the roles played by the researcher. 


\section{CHAPTER 6}

\section{RESULTS - SPECIFIC FACTORS DISCOVERED}

\subsection{Introduction}

The previous chapter highlighted how the actual data was collected, analysed, coded and conceptualised. The chapter also explained how the research was conducted, the research sites, and access to research participants, together with ethical considerations.

This chapter focuses on the findings of this research inquiry. It documents the specific factors discovered in the adoption of ICT in higher education teaching and learning of SIDS such as the Maldives. The specific factors are categorised and discussed according to the contexts of technology, organisation, environment and geography.

\subsection{Discovered factors}

The discovered factors are illustrated in Figure 18. These factors are categorised in terms of:

\section{Technology Context:}

- E-Readiness: Government support for information technology, ICT support for teachers and ICT Infrastructure.

- ICT: Underutilisation of ICT resources, perception of benefits from the present ICT infrastructure, ICT policies, digital divide and ICT confidence among teachers

\section{Organisation Context:}

- Finance, centralisation, human resources, top management support and research

\section{Environment Context:}

- $\quad$ Social Issues: Drugs and overcrowding 
- Culture Issues: Gender disparity and cultural homogeneity among students and teachers and no culture of using ICT in academic learning

- Pedagogy tradition: Spoon-feeding

\section{Geography Context:}

- Geography

- Digital divide

- Transportation

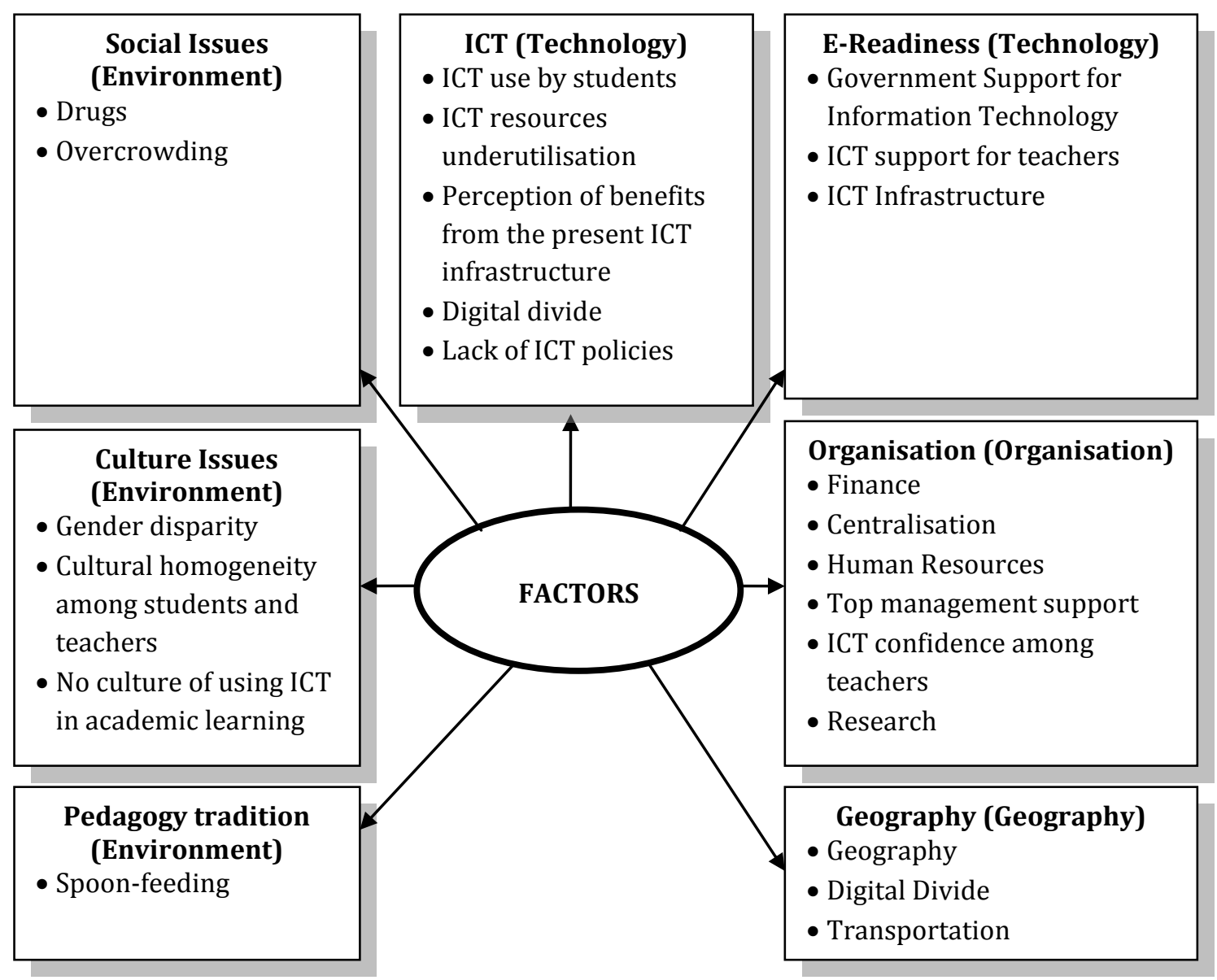

Figure 18: Discovered factors from this research

\subsection{Technology Context}

This section explains the technology factors for ICT adoption within the higher education sector that were discovered in the context of the Maldives. The technology and e-readiness factors include Government Support for Information 
Technology, ICT support for teachers, ICT Infrastructure, ICT resources underutilisation and perception of benefits from the present ICT infrastructure.

\subsubsection{E-Readiness}

\subsubsection{Government Support for Information Technology}

A major initiative by the National Centre for Information Technology (NCIT) is the e-Government project. This e-Government initiative enables the utilisation of ICT services to enhance access to and delivery of Government services to citizens. The e-Government initiative provides ICT services, which reduces the digital divide especially by educating students using ICT facilities (Ibrahim, 2006).

The NCIT is working hard to develop the portals required to provide online information and services to the public. Citizen Centric Solutions (CCS) manage and organise workflow processes, in order to improve communications between Government departments and citizens. This has enabled citizens to gain access to services easily. Two participants interviewed in this study were hopeful of a successful e-Government project:

The e-Government project is to provide the general services required by all the government agencies. We need the authentications and every citizen will have a unique username and password issued by the Government. With the help of this username and password the citizens will be able to interact with the online services available in the Government.

[Information Technology Executive]

The National Centre for Information Technology is doing a significant amount of work in developing and pushing the government network and setting up the government portals. With this e-Government project lot of people will have access and getting access to the government facilities and I am sure people will be more aware of the technological facilities.

[Information Technology Expert]

According to NCIT (2013), one of the objectives of the e-Government project is to provide opportunities for tele-education (see Ibrahim, 2006). Therefore, the eGovernment project in the Maldives will help to strengthen tele-education. The use of tele-education could also make use of existing ICT resources within the higher 
education sector, thus making ICT a medium of communication and sharing information among island schools and tertiary institutes:

Technology is an increasingly influential factor in higher education sector. Computers and mobile phones are used in developed countries both to complement established higher education practices and develop new ways of learning such as online education (a type of distance education).

[Information Technology Expert]

The Government E-letter Management System (GEMS) is making Government administration more efficient, reducing costs, and improving the delivery of services, credibility, transparency and monitoring of the government bureaucracy (NCIT, 2013). The e-Government initiative should develop an ICT-enabled community as a cornerstone of its initiatives to narrow the digital divide that exists between rural communities and Male'.

\subsubsection{ICT support for teachers}

Broadband enabled TRCs have created a single learning community across many islands, to support and link teachers, administrators and students. TRCs have alleviated logistical problems and can decentralise services and resources to reach teachers and students in the remote communities. An Executive in higher education said:

We can utilise the existing TRCs since they are fully ICT-equipped with modern technologies such as 20-25 computers. We should encourage the students to attend these TRCs to utilise the existing ICT resources....

The TRCs are equipped with state-of-the-art technology to improve the quality of secondary and higher education. They have microwave relay and cable Internet equipment which will enable the teachers at tertiary level institutes to use the TRCs to browse the Internet. The existing utilisation of resources within TRCs was raised by the Education Administrator:

TRC's are mainly used for different programs specifically targeted for teachers. For example, in my province forty teachers are taught English upgrading programmes. The majority of the programme is mostly targeted at self-direct learning. Teachers are given some guidance and a little facilitation, then the students use the Internet on their own for online testing practices in English. Students identify their areas of difficulty areas of using and they provide information about their difficult areas where they need help. 
The institutes are unable to cater for the increasing demand from the students, although there are several ICT technologies with high expectations within Maldivian higher education sector. Therefore, there is urgent need to provide courses through technology:

ICT usage is very limited in the Maldivian higher education sector. At the same time I do believe that there is a huge potential in the ICT usage. ... ICT as well as technology usage will grow in the coming years and we will have fully online courses as I mentioned before in the next few years.

[Executive in Higher Education]

Despite having well-equipped TRCs in the islands, there is concern about the utilisation of these ICT resources. The failures of the TRCs are mostly due to lack of skilled human resources working at island level and it looks as if the infrastructure and expensive equipment are being wasted:

The island teachers who graduated from Faculty of Education are using the Teacher Resource Centres (TRCs) which have been is developed with assistance from UNICEF. Some atolls do utilise these TRCs but other atolls since there is no coordinators to assist the TRCs are kept idle.

[Lecturer]

All Atolls have TRCs, but they are not fully functional or properly used for the enhancement and empowerment of teachers' professional development.

[Lecturer]

I did tell you the fate of the UNICEF-sponsored Teacher Resource Centres in the Maldives. I believe you have already interviewed the concerned administrators. It appears that much of the traffic at these Centres is pornography and Job hunting.

[Executive in Higher Education]

\subsubsection{ICT Infrastructures}

A positive driver is the availability of ICT infrastructures in the Maldives. The Maldives does not have an ICT industry given its smallness, but is an ardent importer of ICT products due to high demand from the people. Having ICT infrastructures is important when integrating technology to higher education:

... Significant amount of ICT hardware available, however that is not used to its maximum benefit. Let me specify we do have computer systems virtually in every school and in every college or higher education institution anywhere in the country and even in the smallest of the islands in the school there will be a computer.

[Government Department Executive] 
Availability of ICT resources was highlighted by participants. For example:

If we look at the regional countries, I would consider the Maldives has embraced many technologies in all the industries. If you look specifically at the education sector, school system or even higher education sector we do have the ICT technologies. We are having the ICT infrastructure but we do not utilise these technological ICT infrastructures to the optimum level with huge investments.

[Executive in Higher Education]

There is clear evidence from this study's data that the ICT infrastructures within the higher education institutes are good. An Education Consultant provided promising news, claiming that the majority of the population is now exposed to several technologies but that geography plays a role in gaining access:

IT itself is very strong in the Maldives. I mean IT itself is very strong in the Maldives means that they are quite literate but the vast majority of the population is still being deprived of various IT applications probably because of geographical nature ...

Although there is ICT infrastructure at many institutes, broadening the reliability and affordability of these technologies to all the remote islands is still important:

Access is a fairly big issue in the Maldives. The Internet penetration is high in the urban centres - Male' - but in the atolls the Internet penetration rate is not so high. We need to shorten the digital divide we already have.

[Information Technology Executive]

Multi-purpose Community Tele-Centres (MCTC) will facilitate the provision and expansion of important services, bringing ICT to the island communities at a sustainable and affordable cost (NCIT, 2013). MCTC will help to bridge the digital divide to support e-Government, and open opportunities for islanders to use the ICT resources (InfoDev, 2010a):

Within the school system or even higher education sector we do have the ICT technologies. We are having the ICT infrastructure but we do not utilise these technological ICT infrastructures to the optimum level with huge investments.

[Executive in Higher Education]

It is a fact that all schools and tertiary institutes at least have computers and ICT resources. The equipment is mainly used for classroom delivery and some is used for administrative purposes: 
[There is a] significant amount of ICT hardware available: however that is not used to its maximum benefit. Let me specify ... virtually in every school and in every college [and] higher education institution anywhere in the country and even on the smallest of the islands in the school there will be a computer.

[Government Department Leader]

The Maldivians have the habit of purchasing the latest technologies due to the inherent cultural aspect of competing with each other. Due to the widespread availability of ICT among the people it is cost effective to utilise the ICT resources and ICT they have already purchased:

Maldivians are good at having the most up-to-date technological ICT device with them....

[Education Consultant]

The challenge for educators and academic managers, however, is to understanding and explore how best to use these resources to support learning. Some tertiary institutes have already started delivering information through quick alerts about topics such as rescheduled classes through the SMS facility to student mobile phones.

\subsubsection{ICT}

\subsubsection{ICT use by students}

The qualitative survey undertaken in this research shows in Figure 19 that overall use of the Internet by students was moderately high. The survey showed almost $70 \%$ of the students were using the Internet on daily basis. About one quarter of the students used the Internet several times a day. Very few participants used it only once week. There were no students who did not use the Internet. The findings show participants' Internet usage frequency: 


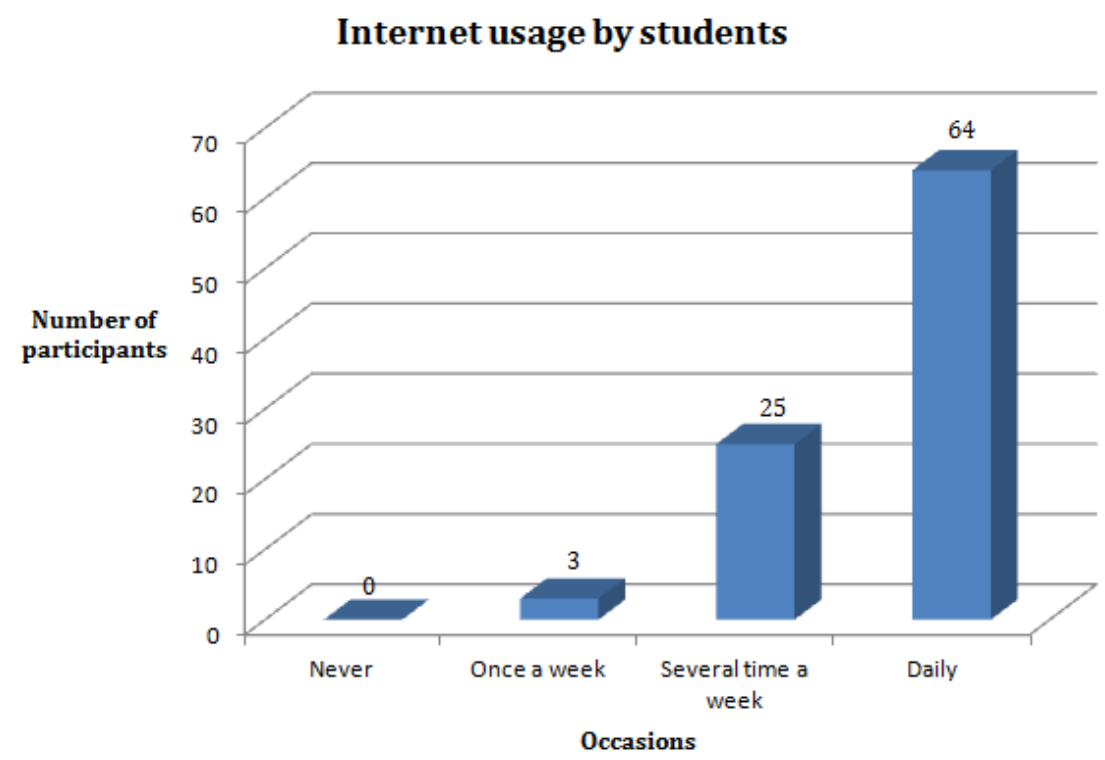

Figure 19: How often do you use Internet?

Figure 20 shows the ICT usage in education by students from the qualitative survey. There were 91 responses from 92 participants. Twenty-seven participants had used ICT for one year; 17 participants for two years; 11 participants for three years; 14 participants for four years and 11 participants for more than four years.

\section{ICT-use by students}

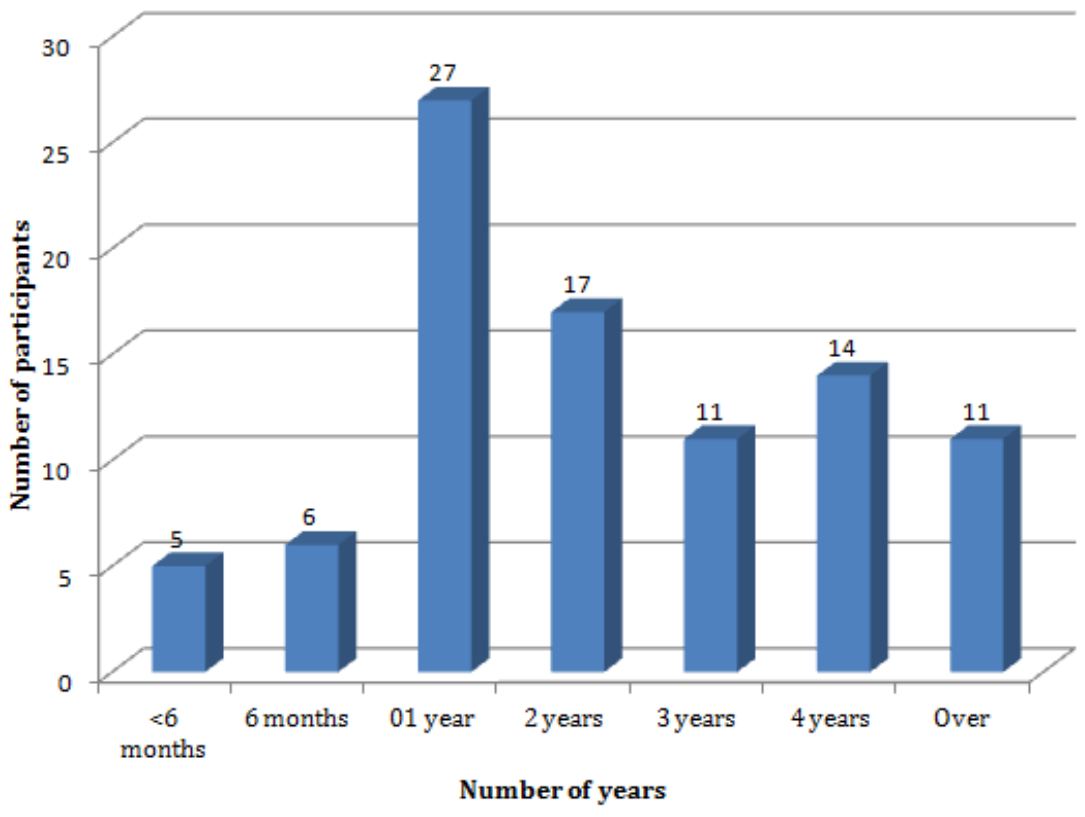

Figure 20: ICT-use by students 
The Maldives has worked over the years to increase citizens' ICT skills and promote more general use of ICT in education, moving away from the traditional exam-focused orientation of instruction (MOE, 2012). These efforts have increased student access to ICT at secondary level, and enhanced knowledge, especially among those who are responsible for increasing the use of ICT.

Currently, for the students at tertiary level, study notes from teachers are distributed either in printed form, softcopies, e-mails or uploaded via a portal. Computer laboratories are provided and students prepare their assignments, assessments and learning materials using these facilities.

Figure 21 illustrates the ICT equipment use by students, according to the qualitative results. The research shows the use of computers in their academic life, out of a total of 92 students. The participants also used other ICT equipment for teaching and learning. About 84 participants listened to lectures where multimedia projectors were used, 85 participants used DVDs, 87 participants used TV and 83 participants used mobile devices.

It is clear from these findings that ICT resources were used by the students. However, it is not clear for what purpose the students were using these ICT facilities. 


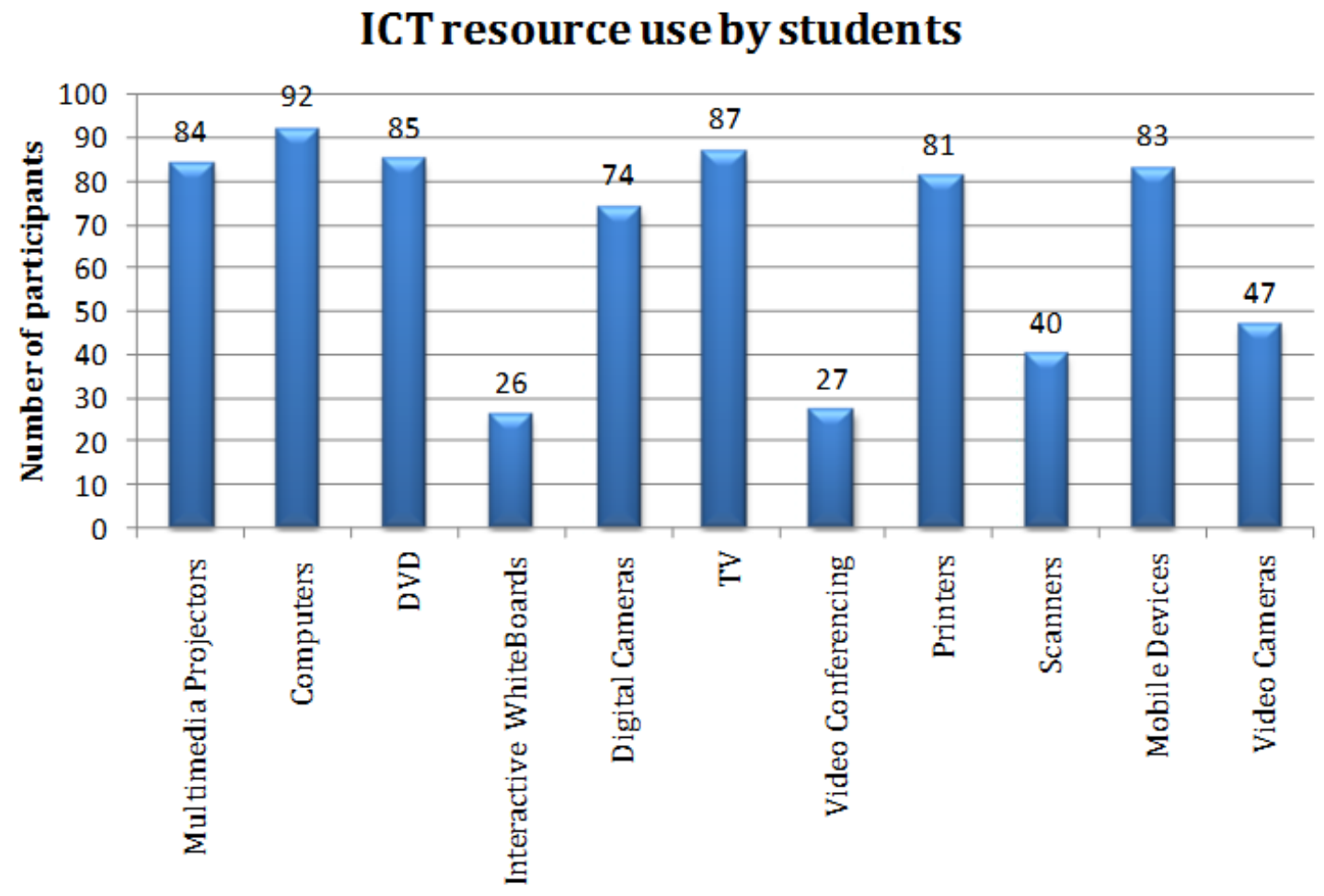

ICT Resources

Figure 21: ICT use as a learning tool by students

\subsubsection{ICT resources underutilised}

There was evidence from the data that the ICT resources were underutilised. The utilisation of existing ICT resources within the tertiary institutes is an important issue to consider. This underutilisation might be due to students and teachers being unaware of the potential of ICT usage. Some were using ICT such as Microsoft ${ }^{\circledR}$ Office package for basic computer operations in their work.

In terms of ICT facilities I would consider the Maldives is far ahead compare to other countries. When we compare with the neighbouring countries, suppose if we look into the quantity and quality of computers and technologies used in the government offices I have to say the Maldives is far ahead. But the difference is how we are using these ICT technological facilities and they are not utilised. Now we face the situation of having the required ICT facilities but lacking how we can utilise these ICT facilities in an innovative fashion.

[Information Technology Expert]

The existing ICT tools were being used to replicate the traditional methods. Most students and teachers were using computers as a device to change existing documents into electronic format: 
Aah! At the moment from my experience ICT is being used more or less as a typewriter. We got a computer for every person generally but they often used [it] as a typewriter for typing stuff and they do not use [it] much [for] teaching and learning except doing presentations.

[Education Consultant]

This is evidence even from the higher education sector, government sector; and private sector, usually computers [are] treated as an extension of a typewriter.

[Information Technology Executive]

\section{A similar statement was made by another interviewee:}

Some instance you will know computers are the most frequent ICT tool and sometimes computers are used as a typewriter. In reality the computer should not be used a typewriter and should be used more significantly because the computer does more things than a typewriter.

[Executive in Higher Education]

Though ICT resources were readily available, there was a lack of skilled ICT personnel on the islands, which worsened the problems. A Government Department Executive said:

ICT usage is used in higher education for word-processing purposes. In reality the ICT usage is unknown or unexplained to the students, teachers or others. This is because the skilled and knowledgeable teachers are not available. We do not have the skilled people to give directives or delegate in the use of ICT.

The latest technologies, especially on the islands, were often untouched because of a lack of ICT literacy or comprehensive support from top management, or teachers were not sufficiently trained to use and teach with them. However, one participant provided evidence that lack of direction from management was another factor for underutilisation:

The resources are underutilised because there is no directive. The computer is mostly used in the Maldives for Internet surfing and they might search some materials for class preparations. But mostly the Internet is used for browsing and for social purposes.

[Government Department Executive]

Higher education institutes failed to take advantage of available ICT resources to develop teaching contents and policies. An Executive in Higher Education commented: 
In fact, the management of MCHE can be run virtually paperless as a result of the investments it has made on ICT over the years. However, the existing Government Policies still require paper trails for many activities of the institution.

Although the computers were not being used to their potential, it is evident from the data that reasonable ICT infrastructures existed within Male' and, together with the reasonable amount in the islands, it should be important to utilise the existing resources.

\subsubsection{Perception of benefits from the present ICT infrastructure}

Almost all academic staff within the higher education institutes of Male' had access to the Internet. ICT can help students in acquiring knowledge and assists teachers and administrators in enhancing teaching and learning. Technology also encompasses the knowledge and skills required to effectively use ICT (Hong \& Songan, 2011).

\section{- ICT as a next generation tool}

Mobile penetration in the Maldives had reached a remarkable high level with 3G and $4 \mathrm{G}$ services launched by Wataniya (i.e. Internet Service Provider in the Maldives) allowing users of $3 G$ services to view video content including music videos (Wataniya, 2011). Youths had a positive perception of new technologies such as $3 \mathrm{G}$ or $4 \mathrm{G}$ and ICT skills enhancement:

Wataniya 3G mobile infrastructures can be used for streaming so easily. This kind of infrastructure can be used to build the learning and teaching and this is a positive driver and we diffuse it. The students (i.e. young crowd) are quite curious to use these kinds of mobile technologies for different purposes.

[Lecturer]

I have to note that our existing 3G services are limited to Male' and to nearby islands (Hulhumale' and tourist resorts) but 3G services are now growing slowly. The 3G services connection speed will be higher than the broadband connection speed. The 3G connection speed would ensure video streaming but will take some time to ensure these services available to the whole country. But I think the $3 \mathrm{G}$ services initiation is very promising for the citizens of the Maldives.

[Student - Focus Group] 
There was demand for the $3 G$ and $4 G$ services among the public in general (Wataniya, 2011). The use of 3G and 4G services should allow students and teachers to actively participate in sessions for learning:

The mobile Internet services have already widened with the $3 \mathrm{G}$ services. The $3 \mathrm{G}$ services ... will increase the speed of Internet connection. With the help of $3 \mathrm{G}$ services via mobiles we can participate in any course at any location within the Maldives. 3G services will also allow us to hear the online lecture and we can communicate with the institutes.

[Student - Focus Group]

The introduction of the $3 G$ network was also emphasised by the former Vice President of the Maldives. He said "still college campuses are very much restricted to Male' and few other islands. But with this new system, many islands that have access to $3 G$ broadband will now be able to access these courses without leaving their islands" (HaveeruOnline, 2009, p.1), a view also reinforced by other participants:

We do have $3 \mathrm{G}$ networks such as video calling and we can use this in teaching and learning and we do not necessarily have to be present in classroom sessions.

[Student - Focus Group]

The Web 2.0 networks allowed video calling, which could be used within the higher education system with the 3G generation of mobile telephony enabling students to ensure maximum benefits for learning

\section{- ICT as a blended learning tool}

A blended learning approach combines both face-to-face instruction and technology-mediated instruction. This approach could enable 24/7 access to all learning content and would ensure equality of access:

An offline model would be good since we don't need an Internet connection all the time and there is a cost reduction. We need the Internet connection at the time of synchronisation and therefore cost will reduce in an offline and online model.

[Student - Focus Group]

The technology-mediated instruction could either be carried out through computers, smart phones, cellular phones, video-conferencing, TV, radio or other emerging technologies:

[Using] offline and online means delivery of training materials that is happening across various [institutes], you know for post-secondary and other training programs.

[Education Consultant] 
The students could participate and engage with interactive learning opportunities in their face-to-face learning sessions. A focus group student shared his/her own experience:

We are building up a system for our final year project - GEM offline system. This would be ideal solution for students in the rural areas and this would be a synchronised system with minimal online time which students can use in offline mode as well. The lecture materials uploaded from Male' can be accessed by the students in the rural islands.

Higher education is primarily based in Male' and there is a knowledge and digital gap between Male' and the remote islands. Access to higher education was a problem in the islands, as shown by this specific example:

... Access to the higher education in the Maldives is very limited. If you live in Male' you have access to higher education but if you live in the further islands then you probably don't have as much access to higher education as you would in Male'.

[Executive in Higher Education]

The blended approach was suggested as a flexible option that could combine faceto-face teaching and learning sessions with a web-based medium:

Using blended learning we can deliver our courses to the rural areas. There are people who are working at the tourist resorts and people who cannot attend a higher education institute due to job commitments and these people can get an opportunity to learn.

[Executive in Higher Education]

It is clear from the data that potential students were stuck on the islands and staff were working in the tourist resorts and were busy and unable to attend tertiary institutes for higher education. The blended approach would be best suited for the Maldives given its constraints:

I think a blended learning approach can combine face-to-face instruction with computermediated instruction which is the best combination based on our geographical situation. It also applies science or IT activities with the assistance of educational technologies using computer, cellular or i-phones or videoconferencing and other emerging electronic media.

[Information Technology Expert]

The islands greatly lacked access to the resources that are necessary for higher education. Textbooks were provided from Male' and getting access to digital libraries would be important to widen the higher education at island levels. There 
was a wider possibility for improving the teaching and learning process, which could be successful with the introduction of ICT:

Giving access to student on the islands who do not have the same access to the library resources as Male' that is also an additional bonus. They do feel that the system has given them wider options. So I definitely feel that ICT is improving and will further improve teaching and learning.

[Executive in Higher Education]

The basic idea of blended learning would provide opportunities for students and teachers on remote islands to make learning more independent and helpful.

\section{- ICT as a video conferencing tool}

The use of video conferencing would allow communications technology to be integrated with video and voice to connect to remote learners (Seenu Hithadhoo campus) on the islands. The hardware required (i.e. computer, webcam, microphone and Internet connections) was available at the tertiary institutes. However, video conference was not being used due to non-availability of human resources or video conferencing centres on the islands:

Video conferencing sessions can be conducted for our programs from FMC and our lecturers can be used for undertaking sessions for students in $\mathrm{H}$. Dh. Kulhudhufushi Campus or S. Hithadhoo campus. These sessions would allow the lecturer at Faculty of Management and Computing at the Maldives College of Higher Education can talk and instant collaboration can take place with far away campuses. Still we are lacking the features to conduct these video conferencing sessions. These new features would enable to increase the richness of courses and improve the standard of courses we are running.

[Executive in Higher Education]

Video lectures and video conferencing facilities were mentioned as two key areas where students could participate interactively in formal lecture sessions:

Video lectures or video conferencing are very appropriate since we are now talking about lack of skilled teachers, lacking subject specific teachers, or lack of teachers. ICT would allow utilising the few teachers we have to conduct video conferencing sessions to a mass audience of students. The teachers do not necessarily limit their teaching to a specific institution and the same teacher can be used by all the institutions or one teacher for the whole country.

[Student - Focus Group] 
The concept is simple as one lecturer could be stationed in Male'. The concept of "one teacher with many students" is important when there is a lack of human resources. The use of videoconferencing would allow the use of telecommunication, both audio and video, which could bring students from different islands together for live sessions. A lecturer in the higher education sector gave his view, maintaining that the "one-teacher-many students" model for video conferencing would be practical, and shared his Australian experience:

I went to South Australia several years ago and they have these Technical and Further Education (TAFE) institutions and I went to this one hall in one TAFE and it is linked to several other remote TAFES in South Australia and it is very remote. This is in a city called Porto Augusta and it is a very small town. The lecturer stands in that room and through that multimedia system he delivers the lecture to six other campuses and they have cameras fixed in all the campuses and everybody in every different campus can ask a question. I think this was in early 90s when I went to Australia. That is a system that we can actually use in the Maldives instead of building campuses and sending a teacher to every single campus.

[Lecturer]

A similar concept could be applied to the context of the Maldives. This concept is would be ideal if one teacher were located in Male' for lecturing and the students on the islands could participate interactively. Video-conferencing could also be undertaken at classroom level with the help of smart boards:

I think when you talked about a model, what comes to my mind is these small places on remote islands. I mean they don't have to be on remote islands, they could be anywhere they could be on the capital too. I see these small places where students gather together under the care of a tutor: one person / one tutor. That is this tutor and he simply facilitates the use of ICT so that these students can learn on their own.

[Executive in Higher Education]

Video conferencing would enable the students to see the teacher so that in turn they could give attention to the lecture:

We don't need to get teachers for each islands and if we can get only one teacher we can pay salary and incentives to this teacher only. I'm saying video conferencing, audio conferencing, and software systems are important ICT tools which can be used in the Maldives.

[Information Technology Expert] 
The students could interact in the session by asking questions and these students might have met before in face-to-face meetings. Video conference meetings would be effective if the students had met before, or meet on a regular basis. No doubt video conferencing could offer solutions for the Maldives' growing challenges and geographical issues:

Better use of video conferencing and other materials can be used in an ICT form. For example: on packs of CDs like that and online exams, so that would certainly improve the learning and teaching. Because right now what is happening is that students who do the courses outside Male' in the campuses they take exams but their marks have to come all the way to Male' to the centre to be processed.

[Lecturer]

Video conferencing and broadband Internet could give higher education for students who were currently denied higher education on the islands:

The video conferencing model would be a feasible solution for us. For example, we have ICT infrastructure at the TRCs. The lecturer can take a session in Male' and with the help of video conferencing the island students can get access to the lecture and participate. The initial cost may be high but the return will be high in long-term.

[Student - Focus Group]

Students could connect with other students on the islands, thereby broadening their knowledge and sharing content. A focus group student also highlighted the need to use video conferencing between Male' and remote islands, since it would be a cost saving exercise:

MCHE have outreach campuses and we can make use of video conferencing facilities whereby we can run sessions in Male' and students from the rural campuses can participate interactively. Video conferencing would be a cost-effective solution for us and a mass audience can participate.

The tele-learners on the islands could benefit from video conferencing because they would be able to live with their families and still study through their computer and video conferencing. One focus group session student said:

Some students do not want to study by textbooks and interactive and interesting video lectures would enable students to learn more. In my experience when I was in Aminiya School (secondary school) when we study by using traditional method of teaching is very boring. But later we studied through videos and it was very interesting for the students. 
The higher education system (i.e. teachers and students) could use course management applications, the Internet, and video conferencing to reduce the human resources:

We are challenged by the fact that we have such limited human resources. And to make existing expertise available over the distributed population, we need to have virtual classrooms and virtual meeting spaces and we need mechanisms by which students can transfer their assignments and work and teachers can transfer their instructions virtually.

[Government Department Executive]

Creating virtual learning environments with the possibility of online or offline approaches would be a practical solution for the Maldives. Video conferencing would allow the creation of "virtual institutes" where academics could work together without the constraint of geographical disparity:

ICT can be used for communicating between learners and teachers like video conferencing can be undertaken with synchronous meetings in a virtual environment or online Forums (also called bulletin boards, discussion boards, or news groups). Blogs (Weblog) are webbased journals and are usually a component of a larger personal or corporate website. Some are more topical and others are highly personal. They typically allow readers to post replies or to be promoted to a co-contributor role.

[Student - Interviewed]

Video conferencing would save a significant amount of money due to the reduction of travel costs. The students could study with peers as if in a face-to-face meeting, which would give them a chance to see one another's body language and facial expressions:

I think it would be the model I described earlier where you link several locations on different islands through ICT and you can have one staff member sitting in one classroom and deliver a lecture or lesson to students located in six different locations. They can all be linked through multimedia systems: kids can raise questions from any location at any given time and the teacher in his own lecture hall can control from where he is going to take the questions.

[Lecturer]

\section{- ICT as a mobile learning tool}

Participants noted that mobile technologies were becoming ubiquitous with enhanced capabilities for teaching and learning: 
[There is] use of mobile technology in teaching and learning within this sector. Hopefully we can have some form of mobile learning within a year or two.

[Executive in Higher Education]

Due to high mobile penetration there was potential to use mobile technologies as an additional tool in the teaching and learning process within the higher education sector:

I think instead of using mobile phones as for basic services you know communication we can use them as teaching and learning process as well, as we have a good number of mobile phone subscribers currently in the country.

[Education Consultant]

We do have the accessibility in mobile telephony and it would be worthwhile to build the learning and teaching environment in this existing mobile infrastructure.

[Lecturer]

According to participants, mobile Learning was a teaching and learning concept that broke the traditional boundaries of education through the use of advanced technologies:

There may be opportunities for us to learn through mobile technologies. For example, we are doing a final project where mobile is used as revision tool. The students can send SMS and get the answers and we can encourage the students to use mobile in their learning process.

[Student - Focus Group]

Mobile technologies were a familiar part of the lives of most teachers and students in the Maldives today. Mobile technologies were the most favoured option, with many people having more than one device:

But still we are very familiar and also we have access to ICT in our daily lives. We have access to computers and we have access to the mobiles. The mobiles that we use in the Maldives are not just ordinary mobiles. Every single mobile can be used to surf the web: we can use them to send text messages and MMS.

[Lecturer]

Interviewee participants expressed the interest among youths in technology. The majority of students were eager to use mobile technologies:

The Maldivian youths are generally very technology friendly. As you know ... everybody is having mobile phones. The youth generation is very keen in using the latest version of mobile phones. The mobiles phones are now used in getting access to Internet.

[Executive in Higher Education] 
The m-Learning project in the Maldives should enable "higher education anywhere" and the new service could enable students across the country to study in "virtual classrooms over Wataniya's $3 G$ network" (HaveeruOnline, 2009, p.1).

There may be opportunities for us to learn through mobile technologies. For example, we are doing a final project where the mobile is used as revision tool. The students can send SMS and get the answers and we can encourage the students to use mobiles in their learning process.

[Student - Focus Group]

The greatest advantage was that students were already familiar with the newest technologies such as $4 \mathrm{G}$ and $3 \mathrm{G}$ networks, which would be a positive sign towards embracing technology quickly:

Students are already quite familiar with computer usage. They know web browsing, searching materials and have mobile phone networks using chat purposes. The students have already embraced ICT but embraced more for socialising needs.

[Executive in Higher Education]

Podcasts enable both teachers and students to listen to educational material both inside and beyond the classroom and advance students' development (Pearce \& Scutter, 2010). The availability of mobile technology would allow podcasting as an enabling technology in facilitating learning through mobile devices (Pearce \& Scutter, 2010; Scutter et al., 2010). The newest technologies such as iPads were being used by students who were ready to adopt ICT as a learning tool (Dale, 2008):

It's possible with the existing technologies in the Maldives, students and teachers can use functions like podcasts for lectures, download tutorials onto mobiles, and undertake video conferencing sessions.

[Information Technology Expert]

There were issues related to podcasting as Scutter et al. (2010) pointed out: that student might no longer attend lectures and disengage from the academic environment:

We need customised solutions for using mobile phones in learning. This will allow the people to use mobile phones even the old people who did not use mobile phones in their entire lifetime. ... We need to cater for the mindset of the people and customise the services from Dhiraagu and Wataniya.

[Student - Focus Group] 
The mobile technology was now playing a key role in the education sector by enabling students to access the Internet, even in remote islands, through using mobile broadband or WiFi:

The mobile technologies for example and use of laptops among students and others have increased tremendously. For example, some people are given laptops for birthday presents. It is evident that students are making use of the WiFi zones at MCHE.

[Executive in Higher Education]

\subsubsection{ICT Policies}

ICT policies could ensure better access to various opportunities in all aspects of life such as higher education and the academic administration of institutes. At the organisational level, there was a lack of ICT policies:

There are no ICT policies and guidelines formulated with respect to the unique context of the Maldives. What we see now are ICT policies and guidelines developed from other countries which are geographically different from the Maldives.

[Information Technology Expert]

The Government is unable to come up with such an information technology policy. Lack of policy has hindered the use of ICT, how we can deliver ICT, and there isn't any control mechanism.

[Coordinator]

A specific example where lack of policy restricted academic members from gaining ICT skills or utilising them was the following:

I'm not aware of any ICT policy in the higher education sector. Lack of policy does not facilitate the allocation of required resources nor does the situation allow systematic promotion of ICT usage in teaching and learning. Lack of policy also does not allow academic staff to plan for ICT skills.

[Government Department Leader]

There was clear evidence of interest being taken at national level to use and enhance ICT facilities to provide better teaching and learning environments. The Government was now trying to formulate policy and guidelines. The use of proper policy should come through the e-Government system, which would benefit the institutes:

There is a lack of ICT policies and guidelines relevant to the Maldivian context, but these ICT policies and guidelines are now in the drafting stage and we are moving in the right direction.

[Information Technology Expert] 
The non-availability of ICT policies was identified as being due to lack of awareness among the top management, who did not seem to understand the importance of having policies at national and organisational levels:

I would say the government should have the policy to either provide mandatory, you know applications whenever there is services to use ICT or else encourage, you know conversion of various applications into ICT mode so that people will have no choice more or less get familiarise understand the various IT benefits you know and others in the country.

[Education Consultant]

Findings revealed there was a lack of ICT policies currently in existence but procedures and guidelines were being followed. A Government Department Leader commented:

The other is the lack of policy emphasis on this. As I said before there is no policy specifically on this one although there are certain practices. Certain practices can be translated as policy in practice. But I think a clear policy guideline has to be there in order to promote this sector.

No foreign investor would invest in or build a venture when there was no clear ICT policy. Two participants further explained the concern about foreign investment:

Since we are having this kind of geographical challenges and we do not have proper ICT written policies in place, the foreign investors will not be interested in investing. I would consider this as a barrier since there are no ICT policies.

[Student - Focus group]

The biggest perceived problem is that we do not have information technology policy. Investors are resisting investing since there is no policy and the investor are reluctant to invest because they don't know what will happen to their investments.

[Coordinator]

The lack of sound policies at national level was a major constraint, which limited the dispersal of ICT resources and equipment. A student in the focus group raised a voice regarding the importance of policy formulation:

Since there is no central body in governing the ICT facilities and there are no policies and I would say no foreign parties will invest in the Maldives if policies are not formulated.

\subsection{Organisation Context}

This section provides a description of the organisational factors that were discovered in this research: finance, centralisation, human resources, and research. 


\subsubsection{Finance}

The financial issues were a concern not only for the higher education sector but had also affected other sectors as well. The Government had realised the role technology could play in reducing costs. Despite this concern, the Government had limited finance for ICT improvements. Two interview participants expressed concerns about limited budgets:

The Government authorities have limited finance to improve ICT and there is no budget. Although the initial investment is high, we need to work hard to get the finance and I think it is a problem. There is no support to develop the education sector at Ministerial level; parliament level, or other levels.

[Information Technology Expert]

But due to the financial difficulties we are unable to use ICT resources to the extent that we expect. Instead we are using very limited ICT resources and we can use more widely ICT in the context of the Maldives.

[Executive in Higher Education]

An interviewed student declared:

We don't have money and still the teacher salaries are now reduced. How can the Government be capable financially to carry out such technology project?

\section{An Executive in Higher Education argued:}

As I have mentioned before at country level there isn't anyone who would give financial support for ICT use. There are island about 200 people in islands and we are unable to provide ICT resources to all the islands of the Maldives. This is a barrier in terms of the Maldives.

An Information technology expert provided a specific example where lack of finance had prevented ICT implementation:

And the lack of finance and the high cost of acquiring, installing, operating, maintaining and replacing ICTs is another challenge in our context. Introducing ICT systems for teaching in the Maldives has a particularly high opportunity costs.

It is clear from the data that there was a lack of financial support targeting the students and teachers in all the research and professional development programmes. 


\subsubsection{Centralisation}

The traditional centralised system practices resulted in the rapid development of Male' while simply ignoring the remote islands' development. There was a heavy reliance on Male' for education, health, social, and other services:

If you look at higher education the services are primarily focused in Male' - the capital. The higher education institutes are stressing hard the importance of using Internet in their students' learning.

[Coordinator]

In addition, the centralisation has created a divide in terms of accessibility to higher education between Male' and islands, due to fewer institutes on the islands and atolls:

Access to the higher education in the Maldives is very limited. If you live in Male' you have access to higher education but if you live in the further islands then you probably don't have as much access to higher education as you would in Male'.

[Executive in Higher Education]

The centralisation system led to many difficulties in terms of congestion in Male'. The influx of people from other islands has been a practise for many centuries. Some islands continued to have a certain level of community autonomy even under the centralised government, while others had become very reliant on the centralised government:

The Maldives have a centralised system and all the island communities are slowly migrating to the capital - Male'. The family migration to Male' has now become a problem and congested Male' cannot cater for these migrants. We are helpless now and the situation looks very grim for us. I feel very concerned.

[Information Technology Expert]

The centralisation in the Maldives caused a lot of delays, rather than a decentralised arrangement where each atoll would have its own autonomous system. The congestion on Male' was further amplified by the continuous flow of people from the islands for higher education, health, and social needs:

A lot of people from the islands are coming to Male' for studies. Male' is congested and hardly have any place to stay. The use of ICT would be the only solution for these students to study while staying in their respective islands. As the Maldives is a geographically dispersed island nation I would say ICT is the best tool we can pick.

[Student - Focus Group] 
There were also problems when someone wanted to study; he/she had to terminate his/her existing job and might be without a job after higher studies:

In our old system we got paid leave for studies but now we need a complete job termination when someone wants to study. The paid worker will have a family with a wife and 3-4 children. When we look in economic terms it is a risk to leave the job and attend higher education studies. We have no guarantee that job will be retained after finishing the course since there is huge reduction in jobs. The islanders also should look after their families, come to Male' and also want to study.

\section{[Government Department Executive]}

The education available in the Maldives is, however, limited to primary and secondary education, although there are growing higher education opportunities. Most could not afford higher education and, with increasing number of students and financial problems, the local tertiary institutes were under constant pressure to reduce costs:

Maldives College of Higher Education is also under great pressure to control our costs. In fact, we have taken drastic steps to reduce the cost of teaching. Our libraries do not stay open as they did before. Students are no longer provided with photocopies of readings. They have to download and print them themselves. The more discerning students we get now stress the quality of education. We have been unable to raise our pay to retain the better part-time staff. Many of them have left us to greener pastures.

[Executive in Higher Education]

The youth in society had fewer tertiary level educational and employment opportunities, both in the public and private sectors.

\subsubsection{Human Resources}

Human resources have been a major issue in terms of implementing a technologybased system in the Maldives. The expatriate teachers at all levels were of equal cost or more expensive than local teachers. Even when salaries were set lower, travel and other benefits typically made it more expensive overall to hire expatriates:

Aaah! I think we have to understand that the Maldives is depending a lot on the expatriate teachers and at the same time local teachers. But the teachers within the school system have to be made more aware of the ICT if we're to successfully incorporate them into the school system. So the ICT competency among the teachers is an issue, at the moment. I believe would hinder the process of, you know successfully applying ICT applications.

[Education Consultant] 
The Maldives depended on expatriate teachers due to the unavailability of local skilled personnel (Mohamed, 2006). The majority of these expatriate teachers and local teachers preferred the traditional methods of teaching and were less ICT friendly:

At schools we have more expatriate teachers than the local teachers and the teachers are mostly from India or Sri Lanka. ... They do not know the London EdExcel syllabus. The expatriate teachers have no know-how to operate multimedia projectors and these teachers still prefer chalk and board.

[Student - Focus Group]

The majority of teachers in the higher education sector are expatriate teachers and the amount could be around $90 \%$ and teachers are coming from India and other neighbouring countries. These foreign teachers are not ICT-savvy and I noticed that these teachers are very distant from the newest technologies due to less experience ...

[Information Technology Expert]

The teaching staff were mainly tutoring privately and spent their spare time in the afternoons lecturing. These lecturers were lecturing for extra money due to the low salaries paid by their full-time jobs:

We also have limited time and a lot of our lecturers are part-time teachers. We have not gone into a training whereby the teachers will utilise the existing ICT facilities. We are using multimedia projectors in classroom deliveries but I would regard that we should utilise all the ICT facilities in the classrooms such as Internet and multimedia projectors. We do have a computer server dedicated for teachers only (i.e. academic server), and teachers can store their teaching materials in this server. I have to say this academic server is also under-used.

[Executive in Higher Education]

Many part-time teachers experienced frustration with their computer skills and felt constricted since they did not have time to become involved in utilising ICT or to learn how to use it in their teaching profession. There was no sound initiation from the higher education institutes either:

As the majority of our teaching staff are part-time lecturers, we are finding it very hard to get lecturers to get involved in using ICT in classroom delivery. There are a significant amount of lecturers who are coming to this institute for 2-3 years. But sadly we haven't undertaken any initiation to utilise ICT in the teaching process.

[Executive in Higher Education] 
The sustainability of existing human resources was an issue raised by Information Technology Executive:

The degree graduates are not there, Master's graduates are not there and diploma graduates are also not there but instead people try to go for further studies and basically nobody is retained. If we cannot retain qualified people in their jobs there is no learning knowledge transfer or no development is happening in the Maldives. If we focus on the professional knowledge academic studies, we are finding extremely difficult to get the real output from the qualified pool of people.

To overcome this barrier, many Maldivian students pursued their higher education, including professional qualifications, university degrees and college diplomas, in overseas countries.

\subsubsection{Top management support}

A need for top management support (from the highest to the middle levels of management) and other relevant people is important for technology-based teaching and learning. Failure often results in abandoning technology-based initiatives or management not giving their support because they are not ready to invest.

According to participants, the top management within the higher education sector of the Maldives feared losing their jobs and did not promote technology to enhance their status even if the technology was appropriate:

Well, I am sure the top academic management do not support the use of technology in our education. Top management should be replaced immediately. You know some are old and resist change. The support from the top academic management is lacking and we need to motivate them as well. The resistance from top academic management is fear of losing their existing jobs.

[Information Technology Expert]

Top management commitment would be required to ensure the continuity and sustainability of ICT-based higher education. The top academic posts were usually filled with old people, thus preventing chance of implementing any kind of technology-based systems:

The thinking level will change in the long-run by incorporating the education personnel into this sector. The institutional heads are not freshly educated graduates but old people 
are leading this sector. This generation of people might not favour using the ICT in terms of business return.

[Executive in Higher Education]

The opinions of the research respondents suggested that management lacked the motivation to change the existing method of teaching and learning due to inability to understand the newest technologies:

We do have the people; we have the knowledge; and we have the technology. The main reason for ICT underutilisation in the Maldives because people are not motivated at all. We need to motivate the top management (i.e. senior staffs) staff with the work of IT qualified people or people with doctoral degrees.

[Information Technology Expert]

Uncertainty of new systems compounded the staff to continue with the "old way" to which they had grown accustomed. A specific example was provided by a focus group student:

The top management is very reluctant to face the new technological changes. For example: employees at Directorial Level are finding difficult even to send e-mails or communicate through text messages because they do not have the required knowledge. Therefore, the top management level staffs are reluctant to face the new technological changes because there is a lack of trained personnel (i.e. human resources).

The current processes and systems seemed fine to the top managers, who were opposed to change due to their limited know-how about technology. Top management at institutes underestimated critical issues, according to another example provided by a focus group student:

The top management do not believe that we should be having the activated (original) software. The top management believes that pirated or trail software is $\mathrm{OK}$ and we can do our job. This is not the way we should be using software in teaching and learning for higher education.

\subsubsection{ICT confidence among teachers}

ICT has become part of society for communication between students and teachers, searching for entertainment and education. But the greatest challenge is that the local teachers were lacking the know-how to utilise ICT in higher education, even though they might be using it for social communications:

I also note that most teachers in the higher education sector do not have the know-how nor do they have the information regarding the potential of ICT in learning and teaching. The 
main reason, I think I have pointed out is that the teachers do not really have the knowhow on using ICT for delivery.

[Executive in Higher Education]

Some participants noted that teachers resisted change and were reluctant:

For teachers the preparation of the lecture materials becomes a headache and teacher do not want an extra work or burden. The teachers are confident that when the teachers use the traditional method it is easy to deliver what the students want. We are changing the phrase "resistance to use ICT" to "do not want to use ICT". Certainly, the use of ICT in teacher delivery puts an extra work or burden.

[Student - Focus Group]

There is evidence from the data that there was a lack of skilled teachers as well as a lack of guidance from academic management. There was a lack of clear directives, which led to poor maintenance of ICT equipment and equally contributed to the underutilisation of ICT resources. A focus group student commented:

The students are having these ICT facilities but I think the biggest problem is that teachers are not ICT savvy and are uneducated in delivering. The teachers do not have the required standard or do not have confidence in using the ICT resources.

In order to make teachers more ICT aware, a laptop programme for secondary education teachers' initiative was introduced in 2009, whereby the Ministry of Education provided 500 laptops for teachers (InfoDev, 2010a). The teachers needed to repay the cost of the laptops in the long term on an instalment basis. Teachers working for private institutions were not eligible to apply for laptops in this scheme:

The other thing is the use of laptops among people is extremely high. The Government has already started a program called "A Laptop for Every Teacher" which means every teacher will have a laptop in future.

[Executive in Higher Education]

Teachers had to learn technical skills adequately enough to use ICT effectively, as well as to guide their students to use ICT purposefully. Teachers not only had to become familiar with ICT but also to acquire the pedagogical know-how needed for productively working with the new technology-oriented learning platforms. But this was not always happening: 
First, the lack of awareness ... ICT facility is available but the full knowledge of the potential is still lacking for various staff to maximise. How it can be used specifically for improving teaching and learning, if there is lack of awareness on this one.

[Government Department Leader]

Some teachers sometimes lacked the confidence in utilising technology in the classroom since their students were more technologically aware:

In my opinion the lecturers don't know how to use ICT. For example, a teacher might think when he / she takes the laptop to the classroom he / she might not even know how to connect the laptop. The teacher might think he / she has to connect the laptop with the help of a student and therefore the teachers resist and have less confidence in using ICT.

[Student - Focus Group]

This is a significant cultural point to be noted. In western cultures teachers might be comfortable with handing technical tasks over to a student, but the culture in the Maldives is different:

There is fear among the teachers to use ICT and this fear is inherited from traditional times and teachers are still resisting using a new technology. A change is not preferred because people might criticise, and due to fear and criticism people do not wanted to use ICT at all.

[Student - Focus Group]

The problem is lack of adequate training and lack of understanding. Good realworld connections and primary source materials are only a few of the resources that would enable teachers to provide excellent opportunities for conceptual understanding. These opportunities were not being explored by the teachers:

The teachers are not actually trained to use those multimedia systems, so that is actually one challenge or one problem we are facing. Staff are not fully trained to use the available resources to its maximum capacity.

[Lecturer]

ICT was still seen as an extra or add-on, rather than an integrated resource within teaching; many teachers were still concerned with "teaching ICT" rather than "teaching with ICT":

No foundation of ICT at primary level schooling. At school level we have access to technologies for senior grade students of the schooling. The computer studies is considered as a subject and taught from Grade 8 level and upwards. I would believe the biggest challenge is that there are no computer foundations at primary level schooling.

[Student - Focus Group] 
The main problem was that a teacher might have ICT skills but lack the ICT knowhow to deliver content in the classrooms. Teachers did not just need ICT related skills, but also knowledge of how to use ICT to promote learning:

Most teachers are not really confident with the ICT in terms of ICT literacy to deliver content. ... However, when it comes to deliver through ICT they are not that confident. I do not feel that they do not have the expertise, but I just feel that they are lacking the confidence. It is not a matter of expertise but it is a matter of having the confidence themselves.

[Executive in Higher Education]

In today's modern tertiary education classroom, teachers must have the expertise and confidence in order to provide technology-supported learning opportunities for their students. The resistance was due to poor teacher support in using technology in their teaching work. The benefits of utilising ICT in classrooms are important but were hindered by factors such as lack of ICT skills among teachers.

\subsubsection{Research}

SIDS and developing countries need research in ICT teaching and learning, especially within the higher education institutes:

No research has carried out in this area of ICT use in higher education. The strategic people might think this is important but they don't know how it can be used. We need to do researches and find out what is important now for us and what should we do now.

There are no researches and no master plans at strategic level decision makers.

[Student - Focus group]

The developing countries and SIDS need to access funds to conduct research and therefore sufficient resources of their own are required. SIDS had insufficient resources to attain sufficient standards to undertake research:

In the case of the Maldives although we don't have our own research carried out and even if the research is there it is not shared. There is no exposure in the Maldives to access online libraries and journals even if we are anywhere. I think we should combine all these in order to use in teaching and learning.

[Executive in Higher Education]

The need for research was also emphasised by a student who has interviewed:

There is no research carried out or feasibility studies carried out or not even a requirement assessment study... 
The availability of research materials in electronic format is also important. Higher education institutes must have online databases and access to journals. An Executive in Higher Education said:

We are not information creators at the moment and we do create information little. If we look on the developed countries or other developing countries the research undertaken by these countries are coming to us in printed format. We now have to embrace ICT to transfer information otherwise we will be lagging behind.

\subsection{Environment Context}

This section describes the environment factors that were discovered in this research: social issues, culture context, pedagogy issues and policy.

\subsubsection{Social Issues}

The diversity and complexity of social issues in the use of ICT in higher education have far-reaching consequences. The consideration of social impediments is important when it comes to integrating ICT into higher education.

\subsubsection{Overcrowding}

The overcrowding in Male' was a major concern where people are cramped in a small area with housing problems. This remarkably high population density in Male' negatively influenced the quality of life, accommodation, congestion, poor sanitary conditions and pollution.

The accommodation cost is exorbitantly high. Landlords were renting out their flats and apartments at a high cost, which had never been encountered previously:

... Male' will be regarded as one of the most densely populated cities in the world [but] I don't have the exact statistics.

[Information Technology Expert]

The population congestion in Male' was a challenge to the Government and the focus was on finding solutions to the issue through the development of regional growth centres in selected islands. Two interview participants commented:

Look, at Male' we have so many people and vehicles cramped within 2 square kilometres. It is just too overcrowded. I don't think we can accommodate everyone from the islands in Male' and we have serious housing problems.

[Information Technology Expert] 
The overcrowding of Male' has created a thriving real estate market and demand for houses and flats are at an all time high. The accommodation costs are extremely high and students from the islands cannot afford to live in Male' and finding extremely hard life if living in Male'. Overcrowding does not only affect people living in overcrowded houses, it affects everyone living in Male'.

[Information Technology Expert]

There was a growing danger of children being left behind on the islands because their parents were coming to Male' seeking jobs and money. This was the only choice for the parents to earn money to support their children's education and other basic needs. There were no opportunities on the islands with limited available services:

The Maldives now face a serious problem of island families are migrating to the capital Male'. The migration is happening at an alarming rate and the Government is trying to stop this migration by trying to implement decentralisation projects. The island communities are migrating to Male' due to lack of opportunities in jobs, education, health and other reasons.

[Student - Interviewed]

Island families were forced to leave the islands for better opportunities. The small children were left alone and some parents came to Male' with their elder children who were seeking tertiary education:

The island families are migrating to Male' for seeking higher education opportunities since most tertiary educational facilities are available in Male'. Some members of the island families might already have settled in Male' and islanders have to come to Male' for higher educational opportunities and mostly the whole family will migrate along with children to Male'. The increase in migration of islanders is leading to congestion problems in Male'.

[Information Technology Expert]

However, the Government was now moving toward the decentralisation concept in order to make significant in-roads into promoting increased student learning across the Maldives (Country Report, 2009):

The Maldives have heavy reliance on the capital - Male' due to the traditional centralised system. The higher education institutes and other resources are mainly available in Male'.

[Information Technology Expert]

The Maldives' urbanisation increased all the development in the country, which was mostly taking place in Male' due to the centralised Government system. This 
led many people from the islands to migrate to Male', resulting in an increase in the population of the capital island:

Everybody will not come to Male' and estimated around 1\% will not come to Male' and will stay in their respective islands.

[Information Technology Expert]

These people who migrated to Male' either worked in the Government, private firms or companies, or self-employed jobs. The accommodation costs in Male' were expensive and students had family commitments, which required them to be stationed in Male' for long durations:

There may be a lot of students and teachers who cannot come to Male' due to unavoidable circumstances. There will be lot of students who cannot come to Male' due to problems in getting accommodation in Male'.

[Student - Focus Group]

The low populations on the islands limited infrastructural developments, mainly due to the lack of economies of scale. In addition, it was also impractical to setup small businesses, as shown by the following specific example:

It is not feasible to set-up a computer repair centre in the islands.

[Information Technology Expert]

\subsubsection{Drugs}

There were a large number of secondary school leavers. Most students did not have higher education opportunities due to the limited number of higher education institutes. The islands did not have higher education resources and heavily depended on Male'.

Social problems such as drug addiction were increased due to lack of employment opportunities, lack of higher education opportunities and family issues:

There are serious social issues in the society of the Maldives like there is a huge number of Maldivian youths are now addicted to illicit drugs. This is happening due to lack of opportunities at tertiary level lack of employment opportunities for youth and lack of facilities.

[Student - Interviewed] 
Due to lack of higher education opportunities in the islands, school leavers are prone to illicit activities:

It is sad that the drugs are mainly used by youths and school leavers since these school leavers do not have post-secondary and tertiary educational opportunities. In addition, there is a lack of employment opportunities and have tendency to go for drug use.

[Information Technology Expert]

The drugs users in families created risks and the likelihood that members of the families might develop drug problems simply because they shared the same house:

There must be some way to return the drug addicts to our society since they are also Maldivians.

[Information Technology Expert]

The overcrowding of Male' led to social problems. There was hardly a family in Maldives, in the capital Male' or on any inhabited island, that had not been affected by the drugs pandemic. Families were thrown into confusion by the discovery that their children had developed a problem with drugs:

There are social risks when mothers from the islands are leaving their small children and coming to Male' for studies. The children are exposed to different social risks since the mothers are away from their children. We need to establish a system so that at least the theory part of the course is completed while the mothers stay on their respective islands.

[Student - Focus Group]

The drug usage was not confined to Male' but was also an inhibitor for going to Male' for higher education.

\subsubsection{Culture}

Cultural issues are important when adopting ICT-enabled higher education. Some cultural issues identified in this study included gender disparity, cultural homogeneity among students and teachers, and no culture of using ICT in academic learning.

\subsubsection{Gender Disparity}

The inferior status of women in the Maldives created economic liabilities because some girl students living on the islands might not have the chance to study for higher education. Existing socio-cultural norms and attitudes combined with prevailing traditional resulted in the woman's position still remaining 
disadvantaged in terms of ICT and education and active participation in the development of the nation (Elnaggar, 2008):

The culture has restriction in our society with respect to gender with families moving to Male' with islands getting vacated. The girls have restrictions in our society partly because of Islamic dimensions and females are not allowed to stay alone in Male' due to insecurity.

[Information Technology Expert]

The disparity between girls and boys arose due to socio-cultural factors. Cultural restrictions existed for female students. Parents took care of their daughters in a restricted fashion:

The parents on the island communities are restricting sending their children to Male', specially the girls for higher education, due to the increase in social problems, such as drugs, violence and high cost of living in Male'.

[Information Technology Expert]

There is a gender disparity in our society when it comes to higher education. Lots of girls from the islands are not getting the opportunity for higher studies. They cannot come to Male' due to serious social problems. Parents do not allow them to come alone to Male'.

[Information Technology Expert]

Due to our culture the female students, as I have said, are particularly restricted by parents from going to Male' thus losing the higher education opportunities. The female student would require secure accommodation with more specifically the girls being specially supervised by the parents. The culture in the Maldives does not allow leaving girls and boys alone when they are 18 years old.

[Student - Interviewed]

Although there were a number of institutions offering higher education in Male', women in the remote atolls found it difficult to travel or stay in Male' to obtain these qualifications. The parents found it difficult to send girls away from home to seek higher education:

The male students can come to Male' and they can sleep in the sitting room of a house. But female students cannot sleep in a sitting room because they will need to sleep and stay in a secure room.

[Executive in Higher Education]

In the island communities, men went out fishing during the day and women were responsible for looking after the children's education and the family, and very often the community. This phenomenon remains so even today in smaller island 
communities. Social interactions and the participation of parents and social groups could play a key role in developing knowledge.

\subsubsection{Cultural homogeneity among students and teachers}

The families in Maldivian society have cultural homogeneity. The island communities are small and people within the atolls know each other. Family traditions among the Maldivians were associated with close relationship practices and beliefs handed down from one generation to the next generation:

The Maldivian society is a close society with everyone knowing each other quite well. There are friends everywhere we go. If you go out you will be meeting dozens of your friends and probably some of them might be your close family relatives. ... We are living in a harmonious society since we cannot even keep secrets.

[Student - Interviewed]

Since the islands are small, and given the existence of community closeness, it was impractical to hide stories or secrets. The social connections between remote island communities were also binding. From a country perspective, the close culture was an advantage:

The Maldivian community is unique and we have bonded communities with close interactions. The Maldivians do know each other quite well since it is a close community and I have to correctly say there are no secrets among people and we cannot hide stories and connections between people are extremely good.

[Information Technology Expert]

The students and teachers on the islands lived together in close proximity. It was very easy to know the detailed family histories of teachers and students. This close rapport of knowing each other had effects, such as teachers favouring students:

The unique features about our education culture are things like our students and teachers living in a close proximity. The students know each other, including their friends. The teachers know their peers, including their families. The students know teachers and teachers know students very well.

[Student - Interviewed]

The students at tertiary institutes had an understanding about their teacher's family backgrounds and vice versa. The students and teachers communicated daily. The Maldives College of Higher Education academic top manager described this 
state of affairs as unique and impossible to be applied to larger countries like New Zealand:

We are a gregarious community of people. Contrast this to another country, say New Zealand, where we may find people living away from each other, often in isolated spaces. I wonder whether these characteristics, the national ethos, alienate or encourage learning in isolation.

[Executive in Higher Education]

\subsubsection{No culture of using ICT in academic learning}

The widely-recognised importance of culture for the successful integration of ICT was even more important for the higher education sector. According to participants, it was important to spread an ICT-flavoured culture in the country and more explicitly in tertiary education:

We do not have an IT culture established in order to use ICT technologies in the Maldives.

[Student - Focus Group]

The culture of using information technologies at school level was limited and in most cases it was not happening due to lack of awareness:

But if you look at schools you will still see 20 computers and computers are treated as a privileged tool and will be allowed to be used once in a while. Once again it falls back to the culture.

[Executive in Higher Education]

We don't have the culture to use technology in our teaching and learning. The bottom line is that there is lack of ICT awareness at all levels. Parents also believe that computers are meant for chatting or to view some website, not as an educational tool.

[Executive in Higher Education]

Another Executive in Higher Education also commented:

The students are not using ICT and from students perspective this is happening due to the school systems and arriving at higher education learning in the Maldives. Most students are coming to higher education institutes after finishing secondary school (i.e. GCE A-Level) and after working. Most of the programmes do not have ICT modules.

There was a general concern among the academics about the impact of spoonfeeding structures between the primary and secondary systems, access to technology, and the positive and negative impacts of using ICT:

So once again it is the same culture practised in secondary schooling and especially in the secondary schooling it is primarily concentrated on spoon feeding styles since it is mainly 
based on exams. The whole purpose of studying in secondary schooling is the final exam or Ordinary Level ( 0 -Level) exam and it mostly concentrated on exams and students heavily rely on notes. The students continuously do exam papers over and over again.

\section{[Executive in Higher Education]}

A key role of higher education is to empower students with skills and attitudes, which are essential in the knowledge society. However, access to computers and the Internet did not mean new learning methods would suddenly emerge:

Clique College has provided students with computer labs which have computer systems, Internet, and are networked with other relevant facilities, but no student is using these ICT facilities. We kept the same ICT resources in the library, hoping that they might be able to use facilities during the class hours. There are free materials available online which are relevant to their courses, but the students are also not utilising these resources.

[Executive in Higher Education]

Parents were concerned about exposing their children to the Internet due to its inappropriately explicit materials. This reluctance was further exacerbated by parents totally forbidding their children from participating in Internet discussion forums:

There are cultural issues associated with online participation since some parents totally refuse their children to be exposed to sexual materials in the Internet and this is partly due to strong Islamic dimensions practised among the people. Most students still refuse to participate even in discussion forums since they fear they might be criticised.

[Information Technology Expert]

Social networking websites tended to be used for social communications only and not for academic learning. Intranets and web facilities were available at institutes but students and teachers rarely used these resources:

You can see very rarely, for example, things like blogging, web facilities, use of intranets to provide resources to the students, communicating or arranging interactive facilities. This is happening may be because it is due to our culture of the Maldives. If you look at the webbased technologies used in the developed nations, you will see in the Maldives web-based technologies are used rarely by few people and in few scenarios.

[Information Technology Expert]

There was limited awareness about the web-based technologies to be used in academia among the students and teachers. Despite students having some useful web-based technologies (for example GEM at The Maldives College of Higher Education), still they were hesitant about utilising them. 


\subsubsection{Pedagogy practices}

The typical approach to teaching and learning was traditional means throughout the education system. Traditional teaching is concerned with the teacher being the controller of the learning environment and the students learn as a result of the process. The traditional "chalk-board-talk" dominated the teacher-centred methodology throughout the primary and secondary schools, and even the higher education institutes in the Maldives:

Maldivians believe that modern methods are better than the traditional method of teaching but yet these two methods are both successful ways. The students get bored of the same way of teaching method done by the teacher, which is on the blackboard and listening to the teacher talk while they sit down in class and heat up their chairs.

[Information Technology Expert]

The traditional method is a difficult role for teachers and parents. The teachers and parents forced the students to study harder and probably memorise their lessons. Extreme power and responsibility were held by the teachers and they played the role of instructor. The existing teaching practices in the Maldivian tertiary institutions had an emphasis on whole class teaching with all students engaged in the same work:

The students adhere to the traditional method of dictating lessons from textbooks; dictating notes word-for-word and students are too much acclimatised to these traditional methods. Therefore, embracing a new method or technology-based methods will be difficult because traditional teaching and learning methods are still practised and it is taking a considerable amount of time in switching to a new method.

[Information Technology Expert]

Even though the traditional method dominated the entire schooling system, schools in the Maldives were now equipped with state-of-the-art technology such as interactive whiteboards and multimedia technologies:

Still traditional methods such as chalk and board are practised in the Maldives and I don't think there are plans to abolish the traditional methods and replace them with ICT tools.

[Student - Focus Group]

The traditional methods we were practising are the chalk and board concept. This is because this traditional method of teaching is carved in our styles but the new model will enable the people to get used to it.

[Student - Focus Group] 
In the case of traditional classroom settings, we use chalk and board with lecture notes distributed to the students. Let us see, the total duration for a class is two hours, and within these two hours the teacher will be writing on the board for one hour and the remaining one hour will be teaching. In the case of using ICT we can take the maximum advantage of full two hours in teaching process.

[Student - Focus Group]

The students were preparing for international exams at secondary school and the curriculum was designed for exam-driven approaches. The assessment-driven methods lacked creativity, which was largely ignored in tertiary institutions, thus limiting students' innovative research:

The curriculum is exam-driven, so it is a paper and pencil exam isn't it? So they don't have to produce except for a statistical project in maths, for which also I don't think you need even to use statistical packages.

[Lecturer]

The students were prepared for written exam-oriented assessments rather than skills-based assessments. Teaching was practised by doing exams throughout the academic year. The teaching methods teachers used were the cramming method:

There is no culture existing in primary schooling or secondary schooling. The students are learning assessment-based learning rather than skills-based learning. The students are continuously given exams and the students are preparing for the exams. If we don't use skills the students will not find or explore and experiment concepts. But rather the students are used to the habit of learning through notes.

[Executive in Higher Education]

At primary and middle schooling levels, ICT literacy classes were optional for students. The teachers should have opportunities to develop ICT skills for the development of general pedagogical capability in the classroom settings:

ICT is still not integrated into the teaching process of the Maldives. I would consider that proper steps were not taken in integrating ICT into the teaching process. I think a dramatic change is required in order to integrate ICT into the teaching and change is required in order to properly push ICT into the teaching process.

[Information Technology Expert]

Concern was raised over the quality of learning, which was not happening because both teachers and students struggled to cope with basic ICT issues. On the other 
hand, the private ICT training providers were self-motivated and had launched a campaign to introduce ICT into the primary and secondary schooling:

ICT is not integrated with the educational curriculum. If you look at the existing curriculums, we don't see the ICT components incorporated into our existing curriculum.

[Executive in Higher Education]

\subsubsection{Spoon-Feeding}

The tradition of spoon-feeding (i.e. rote learning) was the norm in the traditional form of learning. Spoon-fed facts, lessons and notes were replicated in the exams. Spoon-feeding materials to students meant that teachers did everything for them or told them everything that they needed to know, thus preventing them from having to think or act for themselves:

The real problem of the traditional method of teaching is preparing for international exams. The students are preparing for international exams and the spoon-feeding style of teaching is very much practised.

[Lecturer]

The teachers are practising rote-learning in order to increase the scope. This is a huge challenge and institutes are trying to spoon-feed to get good grades from these international exams. We are going not for the purpose of learning but we are going more for the purpose of assessment. We are practicing very assessment-oriented teaching and learning.

[Lecturer]

Most teachers would agree that traditional methods of teaching did not encourage students to become independent learners or to take responsibility for their own learning. But instead students depended heavily on lecture class notes and lecturer delivery. Participants commented that the teachers were conservative and did not want to change from the spoon-feeding style of teaching to technologybased teaching:

The traditional methods are practised such as spoon-feeding practises are still dominating our teaching process. The teachers are teaching their lessons in a spoon-feeding style and new methods of delivery are still not practised. The Government does not have the required policies to push the use of ICT in our education system.

[Student - Focus Group]

Use of ICT culture does not exist in the teaching and learning process. The higher education system is heavily dominated by spoon-feeding styles whereby lecturers provide notes, 
distribute printed materials for students, and there is no room for students to explore themselves and this is my experience.

[Executive in Higher Education]

... The same culture practised in secondary schooling and especially in the secondary schooling it is primarily concentrated on spoon-feeding styles since it is mainly based on exams. The whole purpose of studying in secondary schooling is the final exam or Ordinary Level (O-Level) exam and since it is mostly concentrated on exams the students heavily rely on notes. The students continuously do exam papers over and over again.

[Executive in Higher Education]

Spoon-feeding might retard students' creativity rather than encourage them to learn through inquiry, discovery and problem-solving. The spoon-feeding tradition was practised on the islands, since lessons were taken directly from textbooks and dictated notes.

The nature and practice of the role of academic management were primarily based on preparation to acquire good grades in examinations. Spoon-feeding students might be seen as providing resources that they should acquire themselves:

The students are arriving at higher education learning without breaking the spoon-feeding culture practised in secondary schooling. The students are coming from one age to another with the same kind of spoon-feeding expectations.

[Executive in Higher Education]

\subsection{Geography Context}

This section explains the geography related factors that were discovered in the research. The factors are geography, digital divide and transportation.

\subsubsection{Geography}

The geography plays an important role in the context of SIDS such as the Maldives. As one interview participant commented:

Because of the unique geography of our nation, we are forced to use ICT facilities to deliver information to the islands.

[Lecturer]

Due to the geography there are no economies of scale to support conventional higher education services by building a higher education institute on every island. The geographical concern was repeatedly remarked upon by research participants: 
We live on widely dispersed islands and it's very hard for people living on one island to travel to the other island by boat everyday on a daily basis even to attend higher education institutions.

\section{[Executive in Higher Education]}

[The] Maldives being dispersed across the ocean, ICT can best be used in distance learning where the island students can be educated in their own islands without attending to the institutes.

[Survey Participant]

The geographical formation might not necessarily be challenging, but the remoteness has caused severe problems in extending higher education to the islands. Another of the interview participants commented:

Due to the smallness of the islands it's not possible to extend the higher education services to the student populations in the Maldives.

[Lecturer]

There was a high cost of delivering essential education, social and administrative services to people in remote areas. The students who moved from the islands to Male' incurred high costs of living and transport. The only feasible solution would be to use and apply ICT services to deliver higher education from the atoll levels to the island levels:

Ability to reach many students in different locations simultaneously.

[Government Department Leader]

Almost all the islands had primary and secondary schooling facilities, but the majority of the islands did not have tertiary institutes (MOE, 2012). There was little technical expertise available on the islands to cater for the existing infrastructures or cost of transport:

Given the geography it's extremely difficult to maintain the ICT infrastructures in islands since there aren't qualified technicians to repair the machines and the islands solely depend on technicians from Male'.

[Information Technology Expert]

\subsubsection{Digital Divide}

Economic activities were constrained by geography, leading to inefficiencies and diseconomies of scale. In addition, human resource deficiencies were constraints across the entire spectrum of the development process in the Maldives: 
The ICT devices used in Male' are not familiar in the rural communities. Internet facilities are unknown in the rural areas and not utilised and few students who are exposed in Male' are using these facilities in the rural islands.

[Executive in Higher Education]

Male' has all the products, services and technologies but the islanders are vulnerable. The cause and impact of this disproportionate division of higher educational opportunities extended beyond the education sector and related to other economic and social policies:

The rural islanders are unfamiliar with information technology. It could be the geographical nature of this country is the core problem and the rich people are living in Male'.

[Information Technology Expert]

Despite sound ICT infrastructures, inconsistencies between Male' and the atolls are increasing. Extending ICT services to these distant islands was often a timeconsuming and expensive undertaking:

Income disparity exists among most people and I would consider this a factor as well. Everybody will not have Internet connections or computers at home to retrieve information or do research at their individual homes.

[Executive in Higher Education]

These inequalities posed significant challenges to the country, which included rising unemployment, a digital divide, and unequal educational opportunities. The divide was further increasing, due to the large number of school graduates and lack of higher education opportunities, leading to inappropriate social behaviour. Many young secondary school graduates, determined and with high expectations, were not intending to return to their home island since the job opportunities were limited:

When we look down at the way people live and income disparity is quite strong across the Maldives as well. This means you might not expect to have the same sort of income from the people living in urban areas like in Male' across to the islands.

[Education Consultant]

The small size and low concentration of population limited the scope for providing comprehensive social, higher education, and infrastructure services:

We don't have the critical mass to support the conventional higher education system as such because of our lack of strength and our limited capacity. 
The diseconomies of scale acted as a barrier to sustainable socio-economic and educational development in the Maldives:

The geophysical issue is mainly related to lack of critical mass.

[Government Department Leader]

The low populations on the islands limited the infrastructural developments, mainly due to the lack of economies of scale:

If I am to put in everything into perspective, let me compare ourselves to the largest cities nearby like Colombo for example. We are 300 thousand people meaning just one suburb of Colombo scattered over a region larger than perhaps geographically larger than Sri Lanka. But then the actual landscape is so small that our urban centres constituted with the highest population in densities.

[Government Department Executive]

The inherent constraints imposed by the dispersed and small populations adversely affected the provision of infrastructural facilities and services:

The number of students in each island is very much limited and it is very small actually and it is very difficult to provide expensive ICT technologies for each and every island.

[Lecturer]

The inability to achieve even minimum economies of scale made the operation difficult, not only for small scale activities, but also for secondary schools and institutions of higher learning:

The Maldives consist of small islands with small populations dispersed [over] a wide area and this creates a problem of reaching higher education services to the entire population of the Maldives.

[Coordinator]

\subsubsection{Transportation}

The students and teachers were unable to travel to and from the islands during times of bad weather. There were many students who received their tertiary education by travelling to nearby islands. Parents would not allow their children to travel during rough seas:

How can we travel in bad weather? Can you travel? The answer is a No. There are keen students who are deprived of tertiary education due to geographical and social problems.

[Information Technology Expert] 
When the weather became rough, the students and teachers abandoned travelling, which impeded their educational activities. An Executive in Higher Education noted a specific example:

So if you want to setup a higher education institution say, for example in an atoll, say Gaafu Alifu atoll right? You will have this higher education facility located probably on Villingilli and what that means is that the other islands that are in the same atoll will have people who would want to attend these institutions (i.e. institutions of higher learning on Villingilli). But they wouldn't be able to do so because transport is so prohibitively expensive.

Most SIDS are physically remote and vastly isolated; this remoteness forces dependence on transport providers and associated high transport costs (Gaible, 2009a). From participants' perceptions, web-based systems would answer the geographical problems:

Without travelling the only way to distribute the resources on a daily basis and quickly would be with the help of the Internet.

[Education Administrator]

Inter-island transport was costly because of the distances involved. The small numbers of people wishing to travel made scheduled transport services uneconomic. A Government Department Executive commented:

Connectivity is essential and the first mode of connectivity has been transport. But then transport is so expensive because it consumes so much fuel, so expensive that we have to seek other modes of connectivity without physically transporting ourselves. So ICT is a life saver for us.

From participants' responses, it is clear that the high cost of transportation and communication to and from these remote islands was the most difficult challenge faced.

\subsection{Chapter Summary}

The chapter documented the specific factors discovered in this research inquiry. The analysis in this chapter aimed to comprehend and confirm the importance of the factors affecting ICT adoption within the higher education sector in the specific 
context of the Maldives. The factors were categorised using technology, organisation, environment and geography as main elements. 


\section{CHAPTER 7}

\section{ANALYSIS - HOW CAN ICT BE USED IN HIGHER EDUCATION}

\subsection{Introduction}

The aim of this chapter is to answer the second research question of this study: How can ICT be used to address the issues within the higher education teaching and learning of the SIDS such as the Maldives?

In order to address this research question, the researcher used TAM and DOI as two important theories. In addition, a TOEG framework was formulated using the original TOE by Tornatzky and Fleischer. Technology-Organisation-Environment (TOE) framework is comprehensive and can serve as a theoretical guideline for studying factors shaping the value of ICT in higher education. Based on the analysed data in this research, the researcher has added geography as a fourth element to the original TOE framework.

Finally, this chapter extends the TAM of ICT use in higher education into a SIDS model formulated using the TOEG framework, TAM and DOI. The final model produced using the TOEG framework, TAM and DOI could be a sound blueprint for top management decision makers, academic management and teachers at higher education institutes in defining the new strategies and future trends.

\subsubsection{Technology Acceptance Model}

The Technology Acceptance Model (Davis, 1989) is an important information systems theory that models how users adopt technology, which can be applied to how students and teachers come to accept and use ICT in higher education. The model suggests that when students and teachers are presented with a new technology such as ICT, two factors influence their decisions about how and when they will use it: perceived usefulness and perceived ease of use. 
According to the TAM, perceived usefulness and perceived ease of use are beliefs that are presumed to a) influence attitudes toward new technology, and b) mediate the relationship between external and variables and attitudes (Davis et al., 1989). Perceived usefulness is a great influence on attitude towards computer use and behavioural intention which is influenced by attitude towards computer use.

\subsubsection{Perceived Usefulness}

Perceived usefulness is the belief that a technology is beneficial and enhances performance. This is in line with Davis (1989) who stated the perceived usefulness is the belief that using an application would increase one's performance.

The concept of perceived usefulness can provide important information about a) how academic management perceive technology, and b) how students and teachers will continue to use technology. Teachers' beliefs about the usefulness of ICT in teaching are vital for effective use of technology.

As the factor perceived usefulness explains the user's perception that the technology will improve performance (Davis et al., 1989), the perception of how usefully the technology is performing is important for decreasing the time doing the job, efficiency and accuracy.

\subsubsection{Perceived Ease of Use}

Perceived Ease of Use is a vital belief when intending to adopt ICT within the higher education system since the technology is free of effort. Perceived Ease of Use explains the user's perception of the amount of effort required to utilise the system or the extent to which a user believes that using a particular technology will be effortless (Davis et al., 1989).

The factor of Perceived Ease of Use can provide important information about a) individual differences such as computer self-efficacy and computer anxiety (i.e. resistance to change); and b) individual characteristics such as attitudes towards change or resistance to change. 


\subsubsection{Attitude towards use of technology}

The attitude of top management, students and teachers towards the use of ICT in higher education are a major determinant in whether they actually use or reject ICT. Acceptance of using ICT-based higher education is important to enable the utilisation by students and teachers.

\subsubsection{Behavioural Intention of use}

Behavioural intention is the interest of students and teachers in using ICT in higher education in future. The students and teachers intentions to adopt using ICT are an important factor in the TAM. The pedagogical styles of teaching and learning and culture homogeneity are aspects that are relevant in this research, influencing behavioural intention to use.

\subsubsection{Diffusion of Innovation}

Diffusion of Innovation in organisations (Rogers, 2003) is used in the context of innovation and the TOE framework for studying contextual factors. Diffusion of Innovation has the following three components: Individual Leader Characteristics, Internal characteristics of organisational structure, and External characteristics of the organisation (Rogers, 2003).

\subsubsection{Individual leader characteristics}

Individual leader characteristics such as attitude towards change and resistance to change are important for ICT adoption in higher education. Top Management in higher education institutes are the people who formulate strategic plans but may have insufficient long-term know-how to make sense of the new technologies for themselves. Therefore, ICT awareness among top management is vital for ICT adoption in higher education.

\subsubsection{Internal characteristics of organisational structure}

The research revealed there was a centralised system of higher education system practised in the Maldives. This centralised system inhibited the spreading of higher education to the distant islands. 
The interconnectedness or homogenous culture was an important aspect discovered in the research. There was closeness among families and people knew each other due to the small communities. The interconnectedness between actors such as the higher education institutes was also important. The close interactions between teachers, students and academic management could play a key role in fostering ICT adoption within the higher education sector.

The organisational slack is again another element that can be applied in this study. The organisation slack had a connection with ICT resource underutilisation as was discovered from this study.

It is evident that the process of diffusion can take place from highly centralised systems to highly decentralised systems (Rogers, 1995). In a highly centralised system innovations should be diffused with a low degree of adoption (Rogers, 2003). A decentralised system has a wide range of power sharing and control among the diffusion system, including peer diffusion of innovations through horizontal networks (Rogers, 2003).

\subsubsection{External characteristics of the organisation}

Degree of openness links with the ICT adoption in higher education. The openness affects whether the optimal higher education decision makers choose to invest to utilise ICT. A degree of openness to technology in higher education would be important to sustain this proposed framework.

There is a greater degree of openness within higher education but this does not mean in practice that many teachers and students are able to avail themselves of these new opportunities. Specifically, teachers may have access to digital technologies but lack the necessary ICT skills or confidence to use such technologies. 


\subsubsection{TOEG Framework}

Figure 22 represents the proposed framework using the original TOE framework to formulate TOEG. The factors discovered from this researcher were grounded in the TOEG framework elements (i.e. technology, organisation, environment and geography). These four groups of factors influenced decisions in assimilating ICT adoption within the context of higher education.

The TOEG framework will be a blueprint for academic top managers, academic policy makers and teachers within the higher education sector. The TOEG framework offers a foundation for academic policy makers to review their existing initiatives and undertake upcoming strategies according to the factors raised. The framework should be of importance in the context of SIDS, which have not yet adopted ICT into their higher educational mainstreams. 


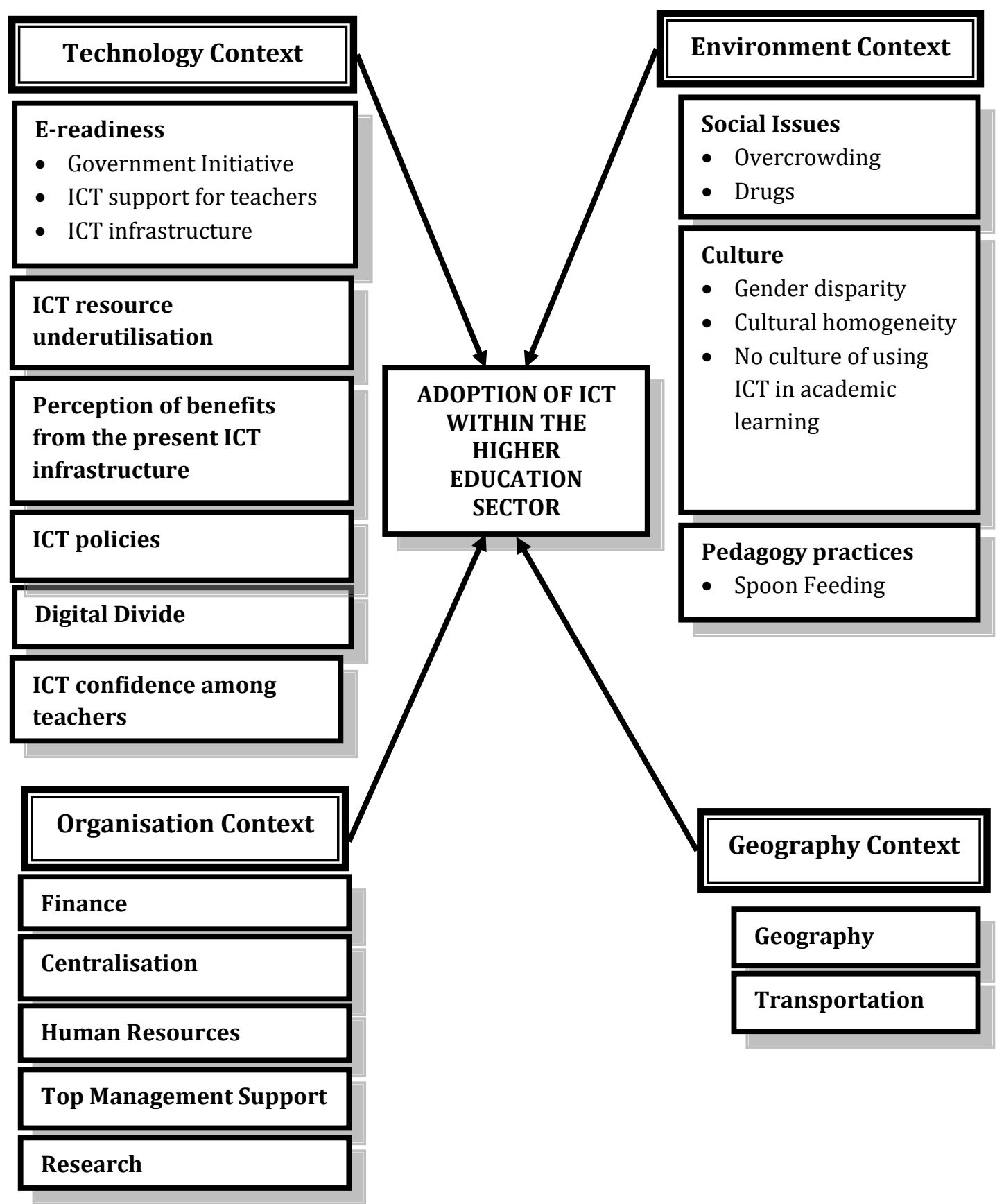

Figure 22: TOEG framework - adapted from original TOE framework (Tornatzky \& Fleischer, 1990)

\subsection{Embedding TOEG framework using original TOE framework}

The TOE framework provided critical elements that are relevant to the use of ICT within higher education. The framework provided a methodical and structured approach in exploring the factors that influence technology, organisation, environment, and geography in the adoption of ICT within the higher education teaching and learning in SIDS. 
From the reviewed literature it was found that the TOE framework was used to examine technology adoption and diffusion. The TOE framework provided a useful analytical framework for studying the adoption assimilation of different types of IT innovations (Oliveira \& Martins, 2011).

Even though the TOE framework has been used in various research contexts, it has not been used extensively to analyse the adoption of ICT within the higher education context. One example was in e-Learning Implementation (Raouf et al., 2012). Raouf el al.'s research used the TOE framework to guide data analysis showing how higher education could adopt and implement the Open Source Software of the Electronic Learning Management System.

The researcher in this study is confident that enrichment of the adoption literature by utilising TOE framework in the Maldives context can provide guidance into how SIDS such as the Maldives could adapt higher education using ICT. Also, the researcher believes the use of the TOE framework in theoretical development strengthens what has been generally neglected in the DOI theory: the specific contexts of technology and organisation.

\subsubsection{Technology Context}

The technology factors identified in the proposed framework were found to be both appropriate and vital for the adoption of ICT into higher education. The technology context describes the existing technologies and new technologies available in the higher education sector.

\subsubsection{E-Readiness}

Government support for information technology: The research data showed the existing ICT structures in the Male' had modern state-of-the-art devices. The use of existing technological resources, such as computers, Internet, multimedia technologies and mobile technologies, in higher education in the Maldives showed a high level of e-readiness that should create opportunities for teachers, students and academic management within the higher education system. 
The e-government initiative seeks to develop an ICT-enabled community in the islands to narrow the digital divide, and bring the facilities, services and opportunities to the people. The current technology projects in the Maldives are eGovernment initiatives for the whole country: Teacher Resource Centres (TRC) in the atolls and Multi-purpose Community Tele-Centres (MCTC) on the islands.

ICT support for teachers: The establishment of TRCs has the potential to improve technology access to the atolls and islands. Some students on the islands had their own computers, while others relied on computer labs at TRCs. The atoll TRCs are a good source of ICT resources, and utilisation of these should be maximised.

Accessibility to TRCs, cybercafés and cyber kiosks is possible in the capital islands of the atolls. The atoll capital islands have stable Internet connectivity but the remote islands lack cyber kiosks and household Internet connections.

ICT Infrastructures: The data showed the most notable feature was that the Maldives had adequate ICT infrastructures in Male' and also on the rural islands. ICT infrastructures include radio, television, Internet, computers, telephony, and smart boards. Every island had good coverage for radio and television.

The speed of the Internet provided by the ISP in the Maldives has increased over the past decade, and could be used for video conferencing on the remote islands to enable students and teachers to see each other, exchange information and transfer knowledge in real time.

\subsubsection{ICT resources underutilisation}

There was clear evidence from the data (refer section 6.3.2.2) that the existing ICT resources were underutilised in the higher education sector of the Maldives. It is clear that higher education institutes wanted to utilise ICT in their teaching and learning. However, challenges such as lack of directives from top management and lack of ICT skills bedevilled their cause. ICT resources have not been fully exploited to address the educational problems that Maldives faces. 
Strong ICT policies should promote the maximum utilisation of ICT resources within each higher education institute. The problem of ICT resource underutilisation should be addressed by top management and policy makers. Top managers should understand that there is no reason to demand new technology if they cannot utilise what is available.

Lack of ICT skills in utilising the existing infrastructure is a significant issue. Skilled human resources are required in Male' and on the islands in order to make best use of the resources.

The cultural issues that prevented teachers from exploiting ICT should also be considered. Teachers' resistance to utilise ICT in classroom delivery was noted in this research.

\subsubsection{Perceived Benefits of using ICT in higher education}

The researcher emphasises the importance of using existing ICT resources within the higher education:

Permanent accessibility (24/7): An ICT-based enabled higher education system would allow students and teachers to have access to resources 24/7. The challenge now was that students and teachers from the islands did not have physical access to textbooks, learning materials or other resources. This permanent $24 / 7$ access might already be available in the remote islands giving access to resources that are unavailable even in Male'.

Direct communication and collaboration: Allows direct communication between students-to-students, students-to-teachers and teachers-to-teachers. This direct communication allows fast and easy interactions. Collaboration in higher education could involve groups of students and teachers working together, including social networking, instant messaging, web sharing, video and audio conferencing. 
New pedagogical methods based on online interactions between students and teachers: Collaborative online communities would provide new opportunities for students and teachers. The ways that students input could be accessed and built upon by other peers, teachers, professionals and experts. These perceived benefits are a direct manifestation of the TAM factor Perceived Ease of Use.

\subsubsection{ICT policies}

The introduction of technology-based systems should result in the development of new ICT policies or utilisation of existing ICT policies. The curriculum should be revised to include courses for students and teachers: a basic ICT-skills and a foundation ICT course. Basic training in Male' could be carried out using tablet applications due to the availability and ease of using mobile technologies. The basic ICT training skills should be undertaken in Male' and the target group would be teachers and academic management.

\subsubsection{Digital Divide}

Geography and socio-economic factors were the cause of the digital divide, and infrastructural and economic gaps exist between Male' and the remote small islands. The remote islanders do not have the same degree of access to modern digital ICT as citizens in Male', and lack the same prospects for social, economic and technology development.

\subsubsection{ICT confidence among teachers}

The teachers possibly resisted technology because they lacked confidence in utilising it. This lack of confidence was due to cultural factors, which made it difficult for them to use technology that students understood and could use better, along with traditional pedagogy.

The teachers lacked the confidence in utilising ICT in the classrooms. Most of the teachers maintained order in the classroom by having a controlled learning environment. Due to lack of ICT knowledge, any attempt in adopting new innovative teaching styles using ICT might be seen as threatening the traditional methods and therefore not desirable. 
Some research participants expressed that there was a genuine fear amongst some teachers about utilising ICT in teaching and disbelief in its value for students. There were often problems with teachers who had some knowledge about ICT but not enough time to plan lessons incorporating their use. Some teachers also lacked the basic ICT skills required.

This low level of confidence in the use of ICT for higher education teaching (and its effects on students' learning) also affects the perceptions about how easy it would be to actually use ICT themselves - the TAM's factor of Perceived Ease of Use. This linkage is described in Figure 24.

\subsubsection{Organisation Context}

It is clear that past research studies utilising the TOE framework used a range of organisational factors. The organisation context describes the nature of characteristics such as finance, top management support; human resources; centralisation and other factors that are relevant.

\subsubsection{Finance}

The lack of finance in higher education (refer section 6.4.1) was a problem found in this study. The high cost remained a drawback for local tertiary institutes. There were financial problems in providing appropriate infrastructures or equipment for students and teachers in the higher education sector.

The required funds were not available and funding was donated from donor organisations, projects or other international organisations. The tertiary institutes did not have an appropriate budget or funds and most institutes were raising their own funds to run their institutions.

\subsubsection{Centralisation}

The educational opportunities were limited within the country and few students had opportunities for higher education. The research participants held the belief that centralisation was the barrier to spreading higher education to remote islands (refer section 6.4.2). 
Within the context of decentralisation, the opportunity to distribute higher education with the help of technology should be introduced soon in the outer islands to give opportunities for students since there was no space in the higher education institutes on the main islands.

Where ICT was used within the higher education sector there was a great challenge of decentralising the services to the remote islands. The decentralisation process sought to increase greater participation of the people (Country Report, 2009).

\subsubsection{Human Resources}

Human resources were an important element in this research. The most prevalent problem in utilising ICT at atoll and island levels was the extreme lack of human resources (refer section 6.4.3). The ICT-skilled people were primarily working in urban Male' and the majority of unskilled labourers were working on the remote islands.

ICT-skilled people would have to be deployed in the atolls, which would need at least a few persons. There should be a minimum of one or two ICT resource people in the remote islands and a few teachers in the atolls (capital islands). ICT-enabled higher education would ensure administrators and teachers could be part of one learning community across the country.

Higher education teachers could simultaneously receive online training for teacher delivery and access and exchange information through the common network. Internet and state-of-the-art technologies aim to increase interactive higher education and to increase motivation of both students and teachers. It would benefit the local populations on the islands, atoll councils and island communities where there was a major lack of higher education due to insufficient human resources.

There was a general shortage of teachers and technical staff, which hindered delivering higher education services to remote islands. The geographical challenge prevented trained human resources from being sent to the smaller populated 
islands. Teachers did not tend to stay on the islands since the income levels and opportunities were low in the rural areas. This barrier could be overcome through the use of ICT to make higher education available on the islands.

The training of teachers and learners should take placed in two streams (i.e. urban and island levels). The training should focus on computer and IT skills and more advanced features. There should be ICT-skilled personnel deployed in the capital atoll islands to ensure that the system is running. Skilled people would naturally be stationed in Male' due to the high salaries and availability of services. The highly skilled people could be deployed as support anchors in the atolls.

\subsubsection{Top Management Support}

ICT-based higher education systems should be implemented in close collaboration with the Ministry of Education, Department of Higher Education and other Governmental organisations. Top management support is a crucial factor in adopting ICT-based higher education. Without serious commitment, the management would not be able to benefit from the technology-based systems in order to enhance institutional learning and teaching processes. Top management had great power to influence the behaviour of other staff members within the higher education institutes.

A strategy should be considered in order to motivate the top management in terms of information technology usage in organisational activities. Through long-term strategic vision and planning, the top management could encourage the higher education organisations to participate in ICT-based higher education systems.

There was a lack of ICT policies at institution and country level. The policy makers at the institutes were the final decision makers and their inability to understand the ICT matters was a major concern. ICT initiatives in the education and other sectors had largely not materialised due to this lack of knowledge and understanding. 


\subsubsection{Environment Context}

The environmental context refers to the arena in which an organisation conducts its operations or services and includes factors such as social issues, cultural impediments; social impediments and pedagogical practices.

\subsubsection{Social Issues}

Overcrowding: The findings showed that overcrowding in Male' caused social problems. The overcrowding was caused by the centralised nature of the Maldives. This not only affected people living in overcrowded houses but everyone living in Male'. The huge migration of people from the remote islands was the main cause of congestion in Male'.

The social impediment factors that were discovered in the findings are important points to consider. The research data (refer section 6.5.1) showed, that parents from the islands were generally hesitant to send their children to Male' due to increasing violence and exposure to drugs in the city. Overcrowding problems caused an increase in crime activities and drugs addicts were also a problem. It is a serious national concern, which affects the country's security and social well-being.

Drugs: Building of effective institutional mechanisms to deal with drug abuse should be grounded in a more rehabilitative approach. This could include provision of easy access to counselling with existing familial ties to deal with new and recovering drug addicts. An education system conducive to producing qualified sociologists, psychologists and psychiatrists was required. Also, ICT could play a role in providing opportunities to bring the drug users back into society through dispersion of higher education to islands.

\subsubsection{Culture}

Gender disparity: The gender aspect is another important area to consider, since the findings showed that there were gender disparities where higher education was concerned. Use of ICT (i.e. Internet and mobile technologies) for teaching and learning would certainly open doors for female students who were stuck on the islands due to cultural and social considerations. 


\subsubsection{Pedagogical practices}

Spoon feeding: There was a tendency to spoon-feed students, since teachers found it a quicker and easier way to prepare students for international exams. The traditional practice at schools entailed students getting good grades by rote learning. At some islands schools and institutes teachers still used chalk and board as the primary tool of delivery tool.

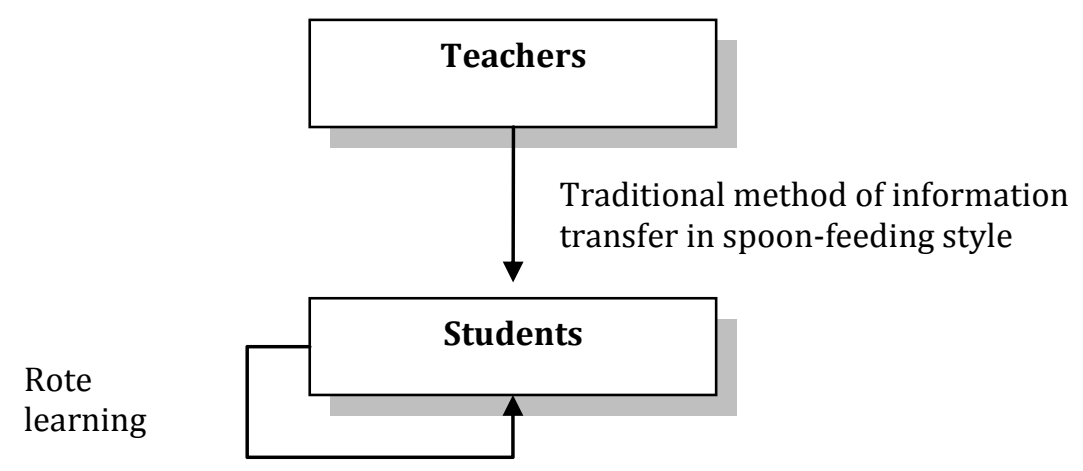

Figure 23: Traditional method of knowledge transfer

Figure 23 shows the traditional model of teaching and learning practised from primary to tertiary education. This concept has been in practice through the education system in the Maldives for ages. The teacher dictates or transforms information through notes and the students listen and take notes in their classrooms. Traditional teaching is concerned with the teacher being the main controller of the learning environment. The traditional teacher-centred methods focus on rote learning, memorisation and spoon-feeding. The implications for ICT would be that students lacked creativity, which might prevent ICT use for research.

\subsubsection{Geography Context}

As the findings have shown in chapter 6, geographical factors were important to consider in relation to ICT adoption within higher education. Geography is a component new to the TOE framework. The geographical separation of islands might benefit from the use of ICT in by making higher education services available to islands' student populations.

\subsubsection{Geography}

The researcher regards geography as the central point. The existing higher education system was unable to deliver higher education to the islands. The 
findings also showed that geography aggravates factors such as human resource development, ICT resources, infrastructure allocations and socio-cultural issues.

The geographic formation of the islands, together with their small populations, was not conducive to achieving economies of scale in higher education and economic infrastructures. This increased the unit costs, creating additional weight, both administrative and technical, which made it operationally difficult to provide higher education services.

\subsubsection{Transportation}

Transport costs were high for students who did not have access to quality institutes on their island, which meant receiving higher education became expensive. With almost $99 \%$ of the territorial area being the ocean, the main form of transport between the islands is by sea (Shareef \& Kinshuk, 2002). Transport has been one of the most significant barriers to dispersion of higher education in the Maldives. Rural teachers and students were unable to travel to Male' and between islands due to high prices, lengthy durations, perceived risks and lack of transport services. 


\subsection{Extended TAM of ICT use in higher education in SIDS model}

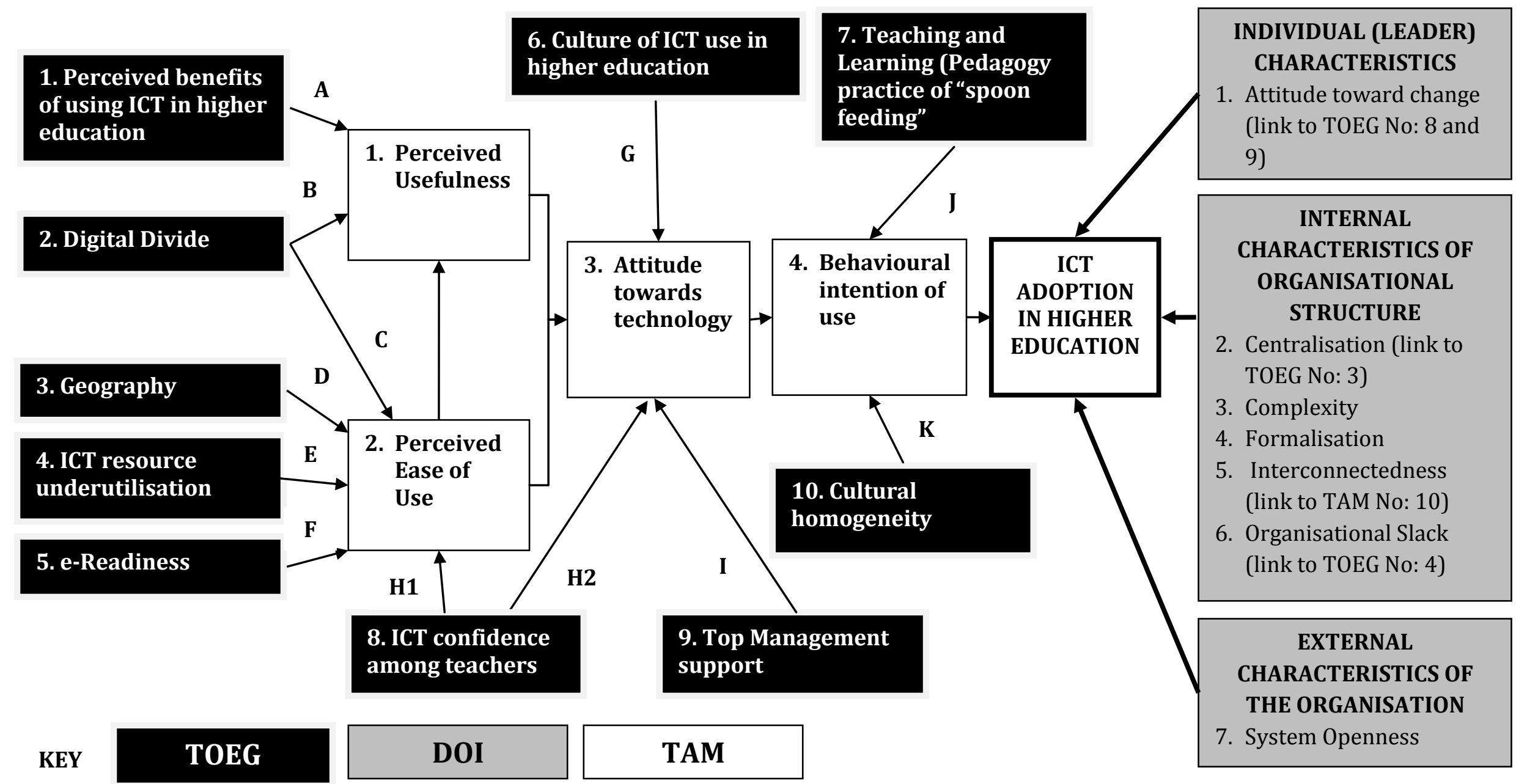

Figure 24: Adapted TAM using TOEG framework and DOI theory 


\subsubsection{Framework Illustration}

Figure 24 shows an extended TAM of ICT use in higher education in SIDS model. To arrive at this model, the researcher applied the TAM theory, TOEG framework and DOI theory. The original TOE framework is consistent with the Diffusion of Innovation theory, in which Rogers (1995; 2003) emphasised individual characteristics, and both the internal and external characteristics of the organisation, as drivers for innovativeness (Oliveira \& Martins, 2011). Several authors used the TOE framework with other theories to understand IT adoption (Awa \& Ukoha, 2012; Hsu et al., 2006; Oliveira \& Martins, 2011).

Rogers (2003) theory of Diffusion of Innovation offers a holistic insight into organisational adoption of innovation and is suitable to consider the problems around the adoption of emerging technologies. The components in Diffusion of Innovation have similar features to the technology and organisational contexts of the TOE framework. The original TOE framework has two components, which are new to DOI (i.e. environment and geography). However, the environmental context presents both constraints and opportunities for technological innovation (Oliveira \& Martins, 2011).

The use of the TAM theory enabled the researcher to use the components to combine with diffusion of innovation and TOEG framework. TAM is a wellregarded theory in Information Systems and is an important theoretical tool for technology acceptance. The TAM is a robust model that has proven useful in explaining and predicting technology use behaviour where social and situational contexts in which technology is overlooked (Venkatesh et al., 2003).

The researcher conceptualised the model by applying the main elements of TAM theory such as Perceived Usefulness, Perceived Ease of Use, attitude towards technology and behavioural intention of use. The factors of the TOEG framework are then further added. The DOI theory is placed on the right hand side of the model in the adoption of ICT in higher education. 


\subsubsection{Technology-Organisation-Environment-Geography (TOEG) framework}

The researcher introduced geography as a fourth component to the TOE framework. Geography played an important role in the adoption of ICT within the higher education of the Maldives. Geography is a basic element for countries with small islands dispersed over a wider area. The researcher believed that this has added value to the original TOE framework and creates a new understanding about the contextual factors in the TOEG framework.

\subsubsection{Technology factors}

The technology factors identified in the TOEG framework integrated with TAM and DOI in Figure 24 are relevant and appropriate for ICT adoption in higher education in the Maldives. The technology factors are the perceived benefits of using ICT in higher education, digital divide, ICT resource underutilisation, e-readiness and ICT confidence among teachers. The links in Figure 24 are explained below:

\section{Link A: Perceived benefits of using ICT in higher education have a direct influence on Perceived Usefulness}

When the Perceived Benefits of using ICT increases, the Perceived Usefulness also increases. In this case, the perceived benefit is being able to study remotely, so the usefulness of ICT is evident. For example, the utilisation of ICT within the higher education sector could create opportunities for the students in remote areas. One student interviewee said:

If the students in the islands have the opportunity to study they can study while at home by online means.

Also an information technology expert mentioned:

Not every student [has] the opportunity for tertiary education, especially students in the islands. The technology based learning can open doors to all the students who are living in the islands. The islanders have fewer opportunities and ICT will open opportunities and there is no need to come to Male'. 


\section{Link B: Digital divide has an influence on Perceived Usefulness}

Narrowing the digital divide could improve the understanding of utilising ICT in higher education and would enhance the performance and improve higher education and thus its perceived usefulness. Perceived Usefulness of ICT is important in order for individuals to access and use information devices in the information society.

The perceived expectation of utilising ICT will be an important precursor to technology use because students and teachers may be more likely to engage in behaviours that they expect will solve problems or result in favourable consequences. For example: a study carried out by Doh and Stough (2010) showed that perceived usefulness of ICT has a direct influence on the digital divide between disabled and non-disabled people (Doh \& Stough, 2010).

\section{Link C: Digital divide has an influence on Perceived Ease of Use}

Perceived Ease of Use (PEOU) can be considered as the frequency of using and the intention to use ICT. There is an inverse relationship, with people in Male' having a high PEOU because Male' is on the right side of the digital divide. In contract, remote islanders experience the digital divide, and have a low PEOU. Empirical evidence in previous Information Systems research has shown that perceived ease of use is significantly related to ICT use intention (Venkatesh et al., 2003).

In the case of the Maldives, almost one third of the population are Internet users (Internet World Stats, 2013). It is likely that higher education students in Male' have good knowledge and experience whereas remote islanders have less knowledge in utilising ICT. Due to remote islanders' relative unfamiliarity with ICT, PEOU is likely to be an important aspect of consideration when it comes to the use of teacher resources centres and multi-purpose community tele-centres in the islands. Therefore, PEOU is likely to be an important aspect of consideration when it comes to the use of ICT in higher education in the Maldives. 
Link E: The underutilisation of ICT resources and Perceived Ease of Use are linked.

With the availability of ICT resources in the context of the Maldives, the focus should be how these resources can translate into meaningful benefits within the higher education sector. Rogers (2003) found that PEOU is an important technology characteristic that generally influences innovation diffusion. For example, better ICT resource underutilisation in the higher education sector could enhance teaching and learning for teachers and students. The need to utilise the existing ICT infrastructure was expressed by research participants:

There is good infrastructure already setup and we can utilise these. In the worst case scenario even if we don't have the proper ICT infrastructures still we can use the existing ICTs.

[Student - Focus Group]

\section{Link F: E-readiness or technology readiness has a direct influence on Perceived Ease of Use.}

E-readiness is the tendency of a community to embrace and utilise new technologies to fulfil goals and is related to the degree to which a particular system would be free from effort. E-readiness explains the level at which SIDS may be prepared or willing to obtain the benefits that arise from the digital economy. For example, there should be a strong chance to embrace ICT since there are already available resources in higher education. When e-readiness increases, the Perceived Ease of Use also increases. One student interviewee explained some benefits of utilising ICT:

The e-Government programme enables connecting all the Government offices. This eGovernment project would allow in sharing information, sharing resources, sharing documents, letters etc. ... The e-Government project would largely benefit to the higher education sector.

\section{Link H1: ICT confidence among teachers is related to Perceived Ease of Use of TAM}

The less confident teachers are with ICT use in their teaching, the weaker their perception of the Ease of Use of the technology and vice versa. The more difficult the ICT is to use for their teaching process, the less confident they will be to actually use it themselves. One Executive in Higher Education observed: 
“... when it comes to deliver through ICT they are not that confident. I do not feel that they do not have the expertise, but I just feel that they are lacking the confidence. It is not a matter of expertise but it is a matter of having the confidence themselves"

A more specific example is where the higher education system changes along with the new pedagogical-style classrooms using ICT. The pedagogy will change when teachers have to use technology in their classrooms. Under these new pedagogical tenets the teachers can act as mentors who provide students with the skills and confidence to conduct their own learning processes.

\section{Link H2: ICT confidence among teachers is related to their attitude towards technology of TAM.}

This research showed that teachers resisted utilising ICT in the teaching delivery process. Teachers' attitudes depended upon how easily they perceived ICT could be utilised at a personal level as well as for teaching in the classroom.

Teachers' attitudes also depended upon how easily they perceived utilising ICT at a personal level and for teaching. Davis et al.'s (1989) model showed that ease of use and perceived usefulness can have a positive influence on teachers' utilisation of ICT.

The more positive the factors such as perceived usefulness and perceived ease of use, the more positive the attitudes of teachers towards ICT and the more likely they are to utilise it in the teaching process. For example: teachers resisted utilising ICT in classrooms since their status might be affected by students being more technologically-savvy. Two research participants expressed their own viewpoints:

The students are having these ICT facilities but I think the biggest problem is that teachers [are] not ICT savvy and [are] uneducated in delivering. The teachers do not have the required standard or do not have the confidence in using the ICT resources.

[Student - Focus Group] Most lecturers with information technology minded young lecturers are using IT [but] some non-IT teachers do not use any kind of ICT due to huge resistance because they get frustrated in front of IT friendly students.

[Information Technology Expert] 


\subsubsection{Organisation factors}

The organisations factors highlighted in the TOEG framework, integrated with TAM and DOI, in Figure 24, relate to top management support.

\section{Link I: Top management support has an influence on attitudes towards technology of the TAM.}

This research showed that there was a lack of support from top management in harnessing higher education initiatives using technology. The top management support in the TOEG framework has a relationship with attitude towards change in the TAM and a connection with attitude towards change in DOI. For example, top management support would depend on managers' attitudes towards ICT use in higher education. An information technology expert expressed the following thoughts:

The top academic posts are mostly filled by old people and they have a tendency to use the traditional methods of working and have disrupted [major] opportunities for institutional development.

\subsubsection{Environment factors}

The environmental factors were (Figure 24) were equally important in addressing the challenges faced in the external environment. The environmental factors were the culture of ICT use in higher education, teaching and learning (pedagogy practices i.e. spoon-feeding) and cultural homogeneity. The relationships between the TOEG factors and the TAM elements are explained here:

\section{Link G: The culture of ICT use in higher education has a link to attitude towards technology of the TAM.}

The culture of ICT use in higher education might be geared to societal changes. For example there was no existing culture of using ICT in academic learning in the Maldives. A focus group student stated:

There is also no culture embedded in using ICT [in higher education institutes] and this is the reason why people are finding difficult to embrace ICT. 


\section{Link J: Teaching and learning (pedagogical practices) are related to behavioural intention of use in TAM.}

The use of ICT could enhance pedagogy if the teachers in the higher education understood that ICT was another platform to achieve teaching and learning goals. Teaching and learning pedagogical practice had a direct influence on teachers' behavioural intention to use ICT in teaching. For example, it is important to change the practice of spoon-feeding style delivery in the classrooms:

If we can promote a system of learning and teaching which enables the students in the islands to learn while in the islands this will be again another driving force which we can create. The only issue with this approach is that Maldivians are too much accustomed to classroom teaching and some might say spoon-feeding, and introducing an alternative needs a lot of effort.

[Executive in Higher Education] I should assert that higher education institutes should make an effort to break this spoonfeeding culture.

[Executive in Higher Education]

\section{Link K: Cultural homogeneity is related to behavioural intention of use}

Due to cultural homogeneity among students and teacher culture context of the Maldives, social networks are also used by the students from the islands and Male' to promote teacher-parent interactions. A higher education executive said:

It is more to do with our culture and how we have been brought up. Students are hesitant to write something in the public forum where somebody else will see it and get criticism and all that. So we will have to change the thinking process and the culture of learning within our institutions so that they do have the confidence to participate in the public domains.

\subsubsection{Geography Factors}

Figure 24 shows that geography was the most critical element in addressing the challenges in delivering higher education in SIDS such as the Maldives. The relationships between the TOEG factors and TAM elements are explained here:

Link D: Geography has a direct relationship to Perceived Ease of Use in TAM. A strong connection was found between geography and Perceived Ease of Use. It was found that geography was the major impediment in delivering higher education to the remote islands. A solution to the geographic separation would 
enhance performance and improve the higher education services in the remote island. In addition, ICT adoption would enhance the spread of higher education to remote islands. ICT is perceived as easy to use and the geographical challenge would become less of a barrier in delivering higher education to the remote islands. A higher education executive highlighted:

Well you know the geography of Maldives as much as I do. Because of this geographical context ICT is seen as a having huge potential in the Maldives.

\subsubsection{Linkages between TOEG and DOI factors}

ICT resource underutilisation (TOEG) vs Organisational Slack (DOI): The researcher argues that there is relationship between ICT resource underutilisation placed in TOEG and organisational slack in DOI. In DOI the organisational slack is the degree to which uncommitted resources are available to an organisation (Rogers, 2003). In other words in this research context slack resources could be described as the existing ICT resources within the higher education sector which are underutilised. This study found ICT resource underutilisation had significant influences on the adoption of ICT in higher education. An interview participant commented on the availability of ICT resources and its level of utilisation:

We have the latest technologies but I don't think ICT is utilised in the higher education institutions at that high level by students. I don't think ICT is utilised in teaching and learning and it is not happening at that level.

[Lecturer]

\section{ICT confidence among teachers and Top Management Support (TOEG) vs} Attitude toward Change (DOI): ICT confidence among teachers and top management attitudes and characteristics could play a vital role and create a positive organisational attitude towards the adoption of ICT in higher education. ICT confidence among teachers and top management support and commitment to the innovation would be essential to ensure adequate financial and managerial resources were provided to create a supportive environment for adoption. However, one student interviewee noted:

... fear and resistance to technology by top management and teachers within the higher education sector [are] everywhere except with students. 
Cultural Homogeneity (TOEG) vs Interconnectedness (DOI): The data showed the cultural homogeneity existed between students and teachers living in the remote islands with sharing of ideas generally leading to greater interconnectedness and ways of life in remote communities. New ideas flow more easily among organisations when there is high degree of network interconnectedness (Rogers, 2003). Therefore, there is a relationship between culture homogeneity in TOEG and to Interconnectedness in DOI. The cultural homogeneity aspect is documented in Chapter 6 of this thesis (refer to section 6.5.2.2).

Geography (TOEG) vs Centralisation (DOI): The centralisation of DOI links to the geography of TOEG. Centralisation refers to the systems where the power and control are concentrated in the hands of a few individuals (Rogers, 2003). Centralisation is the system of governance with a central body stationed in Male', which has complete administrative power over all of the higher education institutions.

The decentralisation concept is relevant to this context because the administration of higher education services could be delegated to atoll or island level. However, decentralisation would require extra human resources, which would need to be deployed within the atolls and islands.

ICT adoption in higher education vs System Openness (DOI): System openness can help us understand how ICT adoption in higher education could manoeuvre in an open innovation setting. Top managers would need to organise their internal processes to get the maximum benefits.

\subsection{Chapter Summary}

This chapter has presented a framework, TOEG, to explain how ICT could be used, with an emphasis on the existing higher education system of the Maldives and the findings. 
The chapter documented the TOEG framework based on the original TOE framework. The TOEG framework consists of four elements: technology, organisation, environment and geography. The integration of the TOEG framework with TAM and DOI was also presented in this chapter. This framework could be a model for ICT adoption within the higher education of SIDS. The researcher utilised TOEG, DOI and TAM to formulate the model.

In the next chapter the researcher will focus attention on presenting the discussion of this thesis. Key findings and reviewed literature are compared to reveal new findings for this research. Essentially, the discussion chapter 8 explains to the reader what the findings in Chapter 6 might mean, how beneficial they could be and why. 


\section{CHAPTER 8}

\section{DISCUSSION}

\subsection{Introduction}

This chapter has two main sections. The first section reviews the major findings of this research. These findings are then compared with the reviewed literature that is relevant to this study. This chapter discusses whether the findings and results corroborate or challenge existing perspectives.

The second section of this chapter focuses primarily on the implications of the research findings for the use of ICT in the provision of higher education in SIDS. The major themes discovered in Chapter 6 are grounded in UNESCO's continuum model of ICT development and TOEG framework, which provides a practical roadmap for the adoption of ICT within higher education. The practical roadmap can be used in the context of SIDS such as the Maldives.

\subsection{Major Findings}

This research has explored contextual factors in the context of technology, organisation, environment and geography and their influence upon ICT adoption in higher education in the context of SIDS, using the Maldives as a specific case. It is evident from the data that these specific factors are vital to understand the Maldivian context. The findings support the existing literature on the use of ICT in higher education and help in revealing the specific factors paramount for ICT adoption in the higher education sector.

The contextual factors discovered are listed here. The technology context factors were Government initiatives, ICT support for teachers, ICT Infrastructure, ICT resources underutilisation, perception of benefits from the present ICT infrastructure, ICT policies, digital divide and ICT confidence among teachers.

The organisation context factors were finance, centralisation, human resources, top management support and research. 
The environment context factors were drugs and overcrowding, gender disparity, cultural homogeneity among students and teachers, no culture of using ICT in academic learning and spoon-feeding.

The geography context factors were: a) the remoteness of the Maldives' atolls, and b) transportation.

The model was developed by adapting the TOE framework together with DOI and the TAM. The TOEG framework, defines four contextual factors for ICT adoption in higher education (technology, organisation, environment and geography).

The TOEG framework in the previous chapter can be utilised by academic researchers in two ways: a) understanding the factors in the context of SIDS and the challenges faced in the adoption process; and b) understanding how ICT resources can be utilised in the context of higher education.

\subsection{Contextual Factors}

The categorisation of the results of this research confirmed that the technology, organisation, environment and geography contexts all have direct and indirect relationships to the adoption process. In addition, the research has identified geography as an important element. The researcher introduced geography as the fourth element to the original TOE framework. Geography is considered vital since it is a feature common to all SIDS.

Tables 13, 14, 15 and 16 show a comparison of the literature reviewed in this study. The second column labelled "Findings in this research" represents factors that were not discussed thoroughly in the literature, are fairly new and have been extended by this current research. The third column labelled "Recommendations" indicates the recommendations drawn from the new findings in the research context. 


\subsubsection{Technology Context Discussion}

The findings in this research revealed that e-readiness plays an important role in the technology content if ICT is to be integrated within higher education in the Maldives. The technology factors identified in the proposed framework were found to be both pertinent and applicable for adopting ICT into the higher education in the context of the Maldives and in SIDS (see table 13).

\begin{tabular}{|c|c|c|}
\hline $\begin{array}{c}\text { THEMES FROM } \\
\text { LITERATURE REVIEW }\end{array}$ & $\begin{array}{c}\text { FINDINGS IN THIS } \\
\text { RESEARCH }\end{array}$ & RECOMMENDATIONS \\
\hline $\begin{array}{l}\text { o ICT Infrastructure and } \\
\text { resources (refer } \\
\text { section } 2.7 .2 \text { ) }\end{array}$ & $\begin{array}{l}\text { E-readiness } \\
\text { o Government Support for } \\
\text { information technology } \\
\text { (refer section 6.3.1.1) } \\
\text { o Good ICT Infrastructures } \\
\text { within the Maldives } \\
\text { (refer section 6.3.1.3) }\end{array}$ & $\begin{array}{l}\text { E-readiness } \\
\text { o Government policies } \\
\text { should support use of } \\
\text { ICT } \\
\text { o Existing ICT } \\
\text { infrastructures should } \\
\text { be utilised in higher } \\
\text { education }\end{array}$ \\
\hline $\begin{array}{l}\text { ICT resources } \\
\underline{\text { underutilised }}\end{array}$ & $\begin{array}{l}\text { ICT resources } \\
\text { underutilised } \\
\text { o ICT resource } \\
\text { underutilisation (refer } \\
\text { section } 6.3 .2 .2 \text { ) }\end{array}$ & $\begin{array}{l}\text { ICT resources } \\
\text { underutilised } \\
\text { o Higher education } \\
\text { institutes should } \\
\text { support the use of } \\
\text { existing ICT resources. } \\
\text { o Higher education } \\
\text { institutes should ensure } \\
\text { that ICT resources are } \\
\text { utilised in teaching and } \\
\text { learning }\end{array}$ \\
\hline $\begin{array}{l}\text { Perception of benefits } \\
\text { from the present ICT } \\
\text { infrastructure }\end{array}$ & $\begin{array}{l}\text { Perception of benefits } \\
\text { from the present ICT } \\
\text { infrastructure } \\
\text { o Perceived benefits of } \\
\text { using ICT in higher } \\
\text { education (refer section } \\
6.3 .2 .3 \text { ) }\end{array}$ & $\begin{array}{l}\text { Perception of benefits } \\
\text { from the present ICT } \\
\text { infrastructure } \\
\text { o Create opportunities for } \\
\text { remote islanders to } \\
\text { engage in higher } \\
\text { education utilising ICT }\end{array}$ \\
\hline
\end{tabular}




\begin{tabular}{|c|c|c|}
\hline $\begin{array}{c}\text { THEMES FROM } \\
\text { LITERATURE REVIEW }\end{array}$ & $\begin{array}{c}\text { FINDINGS IN THIS } \\
\text { RESEARCH }\end{array}$ & RECOMMENDATIONS \\
\hline $\begin{array}{l}\text { Policies } \\
\text { o ICT Policies (refer } \\
\text { section 2.8.2) }\end{array}$ & $\begin{array}{l}\text { Policies } \\
\text { ○ Lack of ICT policies (refer } \\
\text { section 6.3.3) }\end{array}$ & $\begin{array}{l}\text { Policies } \\
\text { o Government and higher } \\
\text { education institutes } \\
\text { should formulate ICT } \\
\text { policies } \\
\text { O Top management } \\
\text { should instigate to } \\
\text { formulate ICT policies } \\
\text { in higher education } \\
\text { sector }\end{array}$ \\
\hline $\begin{array}{l}\text { Digital Divide } \\
\text { o Digital divide (refer } \\
\text { section } 2.7 .1 \text { ) }\end{array}$ & $\begin{array}{l}\text { Digital Divide } \\
\text { o Digital divide between } \\
\text { Male' and the rest (refer } \\
\text { section 6.6.2) }\end{array}$ & $\begin{array}{l}\text { Digital Divide } \\
\text { o Reduce the digital } \\
\text { divide by utilisation of } \\
\text { ICT resources in the } \\
\text { remote islands }\end{array}$ \\
\hline $\begin{array}{l}\text { ICT confidence among } \\
\text { teachers } \\
\text { o Lack of confidence } \\
\text { and competency of } \\
\text { teacher (refer section } \\
2.8 .4 \text { ) }\end{array}$ & $\begin{array}{l}\text { ICT confidence among } \\
\underline{\text { teachers }} \\
\text { o ICT confidence among } \\
\text { teachers (refer section } \\
\text { 6.4.5) } \\
\text { O ICT support for teachers } \\
\text { (refer section 6.3.1.2) }\end{array}$ & $\begin{array}{l}\text { ICT confidence among } \\
\text { teachers } \\
\text { o Higher education } \\
\text { institute should create } \\
\text { initiative to increase the } \\
\text { ICT confidence among } \\
\text { teachers } \\
\text { o Teachers need to be } \\
\text { able to experiment with } \\
\text { ICT without feeling that } \\
\text { their status is } \\
\text { threatened if they fail. }\end{array}$ \\
\hline
\end{tabular}

Table 13: Technology context factors

\section{$\underline{\text { E-readiness }}$}

The data showed the need for Government support in implementing information technology initiatives (see section 6.3.1.1). The major information technology initiatives are documented in section 6.3.1.1. In order to implement these initiatives, the Government should put policies in place to support the use of ICT 
within the higher education sector. The data emphasised that ICT infrastructure is a key element in the adoption of ICT within higher education. There were concerns raised about the lack of ICT usage, with research participants highlighting the lack of guiding policies about ICT usage. Ainley et al.'s (2008) study also raised key question for institutes and recommended engaging students with education by using ICT.

The findings and analysed data showed a strong consensus among research participants about the availability of existing ICT infrastructures within the Maldives. This is consistent with the literature (CAM, 2013; ITU, 2010; Internet World Stats, 2013), which showed existing ICT infrastructures were good. ICT resources were reasonable compared to other regional countries (Asian Development Bank - ADB, 2010; InfoDev, 2010a; ITU, 2010; UNCTAD, 2009; UNESCAP, 2008) as well.

Statistics confirmed that, compared with other countries in South East Asia, the Maldives had reasonably good ICT technologies (ITU, 2010; UNCTAD, 2009). Given all the available resources, high Internet penetration rate, mobile coverage and ICT infrastructures in the Maldives (Internet World Stats, 2013), the researcher argues that the existing ICT should be utilised within higher education. The lack of existing ICT resource utilisation was an area of concern raised in this research. The researcher believes that technology alone is not a panacea and there is a need to have skilled personnel to utilise the existing resources at higher education institutes. In order to take maximum advantage, there should be increased utilisation of existing ICT infrastructures within the higher education sector.

\section{ICT resources underutilised}

The most noteworthy points revealed by the research participants were concerns over the issues of ICT resource underutilisation. This research confirmed the availability of ICT resources, on the islands, at atoll level and in the urban areas, but unfortunately these resources were under-used due to the lack of skilled human resources or directives from the top management. 
In addition, some research participants commented that the ICT resource underutilisation occurred because there were no proper ICT policies. The teachers needed to be guided in utilising the existing resources more effectively. Lane (2009) confirmed from an international perspective that "... personal circumstances mean they also lack the necessary skills and the confidence to use such technologies in general or for education in particular" (p.1).

The provision of TRCs in the atolls was a good example of available ICT resources quoted in the research by participants. These TRCs have training rooms, computer labs, smart boards, and broadband Internet, and are strategically placed on the capital island of every atoll. With better use of TRCs, teaching and learning within the higher education system could be interactive and participatory for both teachers and students. The increased availability of personal mobile devices is another factor that should be considered, since these are likely to be a significant part of the ICT resources available to students.

Another new finding at the institutional level was that there has been no research carried out the Maldives to find out why ICT resources are underused. It is also unclear how ICT resources are underused in the context of higher education. However, this study has provided some insights, suggesting that cultural factors might inhibit teachers from adopting ICT.

The concept of ICT resource underutilisation is not novel; as Sutherland et al. (2004) pointed out, there may be problems of ICT underutilisation at institutional level even when the resources are readily available. Appropriate use of TRCs, telecentres, e-Centres and e-Government initiatives are potential areas that could be utilised in future. The higher education institutes should support the use of existing ICT resources in teaching and learning. The researcher concludes that resource underutilisation is a critical factor affecting the adoption of ICT within the higher education sector of the Maldives. 


\section{Perception of benefits from the present ICT infrastructure}

The findings also highlighted that the potential benefits of ICT could be immense for the Maldives due to its geographical aspect and socio-cultural context. This confirms the literature reviewed. InfoDev (2010a) stated that “... the Maldives is an example of a country which can reap great benefits from ICT implementation particularly because of its geographical make up and the Government initiatives" (p.76). Due to its topographical nature, there are logistical problems in providing higher education services to the island communities. The Government would have to inject significant funds to deliver higher education to distant islands, most of which are currently utilised for transportation between islands.

There is a natural separation of ICT into older and more modern technology. The former includes fixed-line telephones, radios and televisions and the latter includes mobile phones, video conferencing, m-Learning, computers and the Internet, and with the existing ICT technologies, the solution should be straightforward. If the students on the islands cannot come to Male' for tertiary education, then the higher education should come to the students.

Technology-based higher education could create opportunities for islanders to learn and gain knowledge while remaining with their families. Placing ICT at the forefront could provide higher education for students who are currently disadvantaged on the remote islands due to geography.

\section{$\underline{\text { ICT Policies }}$}

The findings of this research highlighted the lack of ICT policies (refer section 6.3.3). Not having an ICT-guided policy was a problem in the context of using information technology in the Maldives. The need for ICT policies was raised by the participants. The importance of having policies at national level was also highlighted by the reviewed literature (Adam \& Urquhart, 2007). The need for a national ICT policy would ensure maximisation of the potentials of ICT, by providing over-arching policies, incentives and legislation According to Aneh Dhivehi Rajje (2009-2013), the national education strategies for 2009-2013 
contain a policy related to ICT (referred to Policy 13): "enhance and optimise teaching, learning and management through the use of ICT"' (p.232).

The most important area of consideration should be integration of ICT policies within the higher education sector. Regulatory bodies such as the National Centre for Information Technology in the Maldives could enforce such ICT policies. As this research found teachers were not confident in utilising in classrooms, specific attention should be paid to making teachers more aware of the potential of ICT, and more confident in experimenting with ICT. The Government and higher education institutes should formulate ICT policies in order to implement ICT-based initiatives within the higher education sector.

ICT policy is required to promote teachers' ICT use in order to maximise its full potential in teaching and learning ( $\mathrm{Hu} \& \mathrm{Webb}, 2009)$. In primary and secondary schooling, IT literacy classes are currently optional. Most primary and secondary schools in Male' do have computer laboratories, which are managed by a local company. Regulatory bodies should take the initiatives in ICT policy development, expanding the frontiers of technology education to primary, secondary and tertiary institutions.

The National Centre for Information Technology could facilitate the development of ICT policies at national level, advise the Government on the National IT Strategy, and also review and undertake studies in technology and policy. Top management should instigate the formulation of ICT policies within the higher education sector.

In conclusion, this research has shown that sound ICT policies are vital within higher education institutes and at the national level.

\section{Digital Divide}

The findings supported the literature confirming that the digital gap (Gulati, 2008; Shareef et al., 2010) is ubiquitous between urban and rural areas, which was an expected outcome for this research. The prevailing digital divide problems (Athif \& Pimenidis, 2009; ITU; 2004) brought uneasiness to students, teachers, academic 
managers and parents in remote areas who were unfamiliar with new technologies.

As show in the literature (Dada 2006; Reddi \& Sinha, 2005; Rogers, 2001; Willis \& Tranter, 2006), this research confirmed that remote island communities were usually disadvantaged by the lack of Internet services. However, in the case of the Maldives, a reasonable amount of ICT resources were already available for use within the higher education sector. It was confirmed by the research participants that Male' and the atoll capital islands were equipped with ICT resources and connectivity, which could be utilised for higher education teaching and learning.

Literature also showed that the existence of a digital divide caused students and teachers to lack confidence with the new technology and digital devices (Bingimlas, 2009). The Perceived Ease of Use was that ICT technologies were too problematical or complicated for teachers who were unsure about teaching or how to use them in their teaching. The research showed that teachers (i.e. Late Majority in Rogers adoption curve) and top management (i.e. Laggards in Rogers adoption curve) in who were uncertain or had an ambiguous attitude towards computers, the Internet, and other recent technologies.

The smallness and/or remoteness of the islands posed problems in setting up secondary schools and tertiary institutes. The most daunting phenomenon was the high transport costs in SIDS (Binger et al., 2002; Gaible, 2009a). The Government has promised to establish a nationwide transport system (Aneh Dhivehi Rajje, 2009-2013).

The low populations on the islands meant no economies of scale, making for support for conventional higher education difficult. The majority of islands had a population of fewer than 500 people (Ibrahim, 2006; Shareef et al. 2010). The digital divide problem could be tackled through a four-step process: understanding, access to technology, technology sustenance, and resource people (Herselman \& Britton, 2002). From the analysed data, with reference to Herselman and Britton's four-step process, understanding and access to 
technology would be easy to implement, but the Maldives lacked technology sustenance (i.e. resource utilisation) and resource people (human resources).

The utilisation of existing ICT resources in remote islands would have the potential to narrow the digital divide that exists in the Maldives. In conclusion, the researcher maintains that the digital divide is an important factor when it comes to SIDS.

\section{ICT confidence among teachers}

The findings showed that there was a lack of ICT confidence among teachers (refer section 6.4.5). This compares with other literature, which revealed a lack of ICT competency among teachers (Bingimlas, 2009; Erstad, 2003; Prain \& Hand, 2003; Slay et al., 2008). In a similar manner, Bingimlas (2009) stated that "teachers who resist change are not rejecting the need for change but lack the necessary education in accepting the changes and are given insufficient long-term opportunities to make sense of new technologies for themselves" (p.239). In order to address the lack of ICT confidence among teachers, higher education institutes should create initiatives to increase awareness and knowledge about utilising technology in classrooms.

Teachers need to be confident and able to experiment with utilising ICT in classroom settings without feeling their status will be threatened if they fail. In the case of the Maldives context, the students were more confident than their teachers in utilising ICT in classroom, and teachers were simply resistant. There were limited local teachers but they should be able to experiment with ICT and an awareness programme should be created for them to encourage them to utilise technology in classrooms. The higher education institutes could play an important role in developing initiatives to increase the ICT confidence among teachers.

Rogers' (1995; 2003), DOI theory classified users of innovation into five categories, from innovators to laggards. These categorisations show that there are differences between later ICT adopters' and earlier ICT adopters' personal characteristics. A non-technical approach would be desirable at the institutes, since those teachers 
who are technologically-distant might have more difficulty in adopting ICT (Rogers, 1995; Rogers, 2003).

Complexity from Rogers' five characteristics of adoption of innovation is important to consider here. The teachers' resistance to change might be due to perceived difficulty in understanding the use of ICT. Different people have different approaches to gaining skills and knowledge with any technology. The usage of ICT among learners and teachers required skills and knowledge and time.

In conclusion, ICT confidence among teachers is a vital factor influencing ICT adoption found by this research.

\subsubsection{Organisation Context Discussion}

Based on the findings of this study, the organisation context factors included finance, centralisation, human resources, top management support and research.

The analysis of the empirical data in this research confirmed the strong relationship between the adoption of ICT in higher education and the identified organisational factors (see table 14). 


\begin{tabular}{|c|c|c|}
\hline $\begin{array}{c}\text { THEMES FROM } \\
\text { LITERATURE REVIEW }\end{array}$ & $\begin{array}{c}\text { FINDINGS IN THIS } \\
\text { RESEARCH }\end{array}$ & RECOMMENDATIONS \\
\hline Finance & $\begin{array}{l}\text { Finance } \\
\text { o Finance (refer } \\
\text { section 6.4.1) }\end{array}$ & $\begin{array}{l}\text { Finance } \\
\text { o Higher education institutes } \\
\text { should explore ways to } \\
\text { obtain finance through } \\
\text { international donor } \\
\text { organisations or } \\
\text { Government projects. }\end{array}$ \\
\hline $\begin{array}{l}\text { Centralisation of } \\
\text { Higher } \quad \text { Education } \\
\underline{\text { services }}\end{array}$ & $\begin{array}{l}\text { Centralisation of } \\
\text { Higher Education } \\
\underline{\text { services }} \\
\text { o Centralisation (refer } \\
\text { section 6.4.2) }\end{array}$ & $\begin{array}{l}\text { Centralisation of Higher } \\
\text { Education services } \\
\text { o Government and higher } \\
\text { education institutes should } \\
\text { support the decentralisation } \\
\text { of higher education at atoll } \\
\text { and island levels. } \\
\text { o ICT should be used to } \\
\text { deliver higher education } \\
\text { services to the remote } \\
\text { islands }\end{array}$ \\
\hline $\begin{array}{l}\text { Human Resources } \\
\text { o Human Resources } \\
\text { (refer section 2.8.1) }\end{array}$ & $\begin{array}{l}\text { Human Resources } \\
\text { o Human Resource ICT } \\
\text { skills (refer section } \\
6.4 .3 \text { ) }\end{array}$ & $\begin{array}{l}\text { Human Resources } \\
\text { o ICT skilled people should be } \\
\text { produced and encouraged } \\
\text { to be available in remote } \\
\text { islands to cater for demand }\end{array}$ \\
\hline $\begin{array}{l}\text { Top management } \\
\underline{\text { Support }} \\
\text { o Organisational } \\
\text { Support and systemic } \\
\text { issues (refer section } \\
2.8 .3 \text { ) }\end{array}$ & $\begin{array}{l}\text { Top management } \\
\text { Support } \\
\text { ○ Top management } \\
\text { support (refer } \\
\text { section } 6.4 .4 \text { ) }\end{array}$ & $\begin{array}{l}\text { Top management Support } \\
\text { o Top management should } \\
\text { support the use of ICT at } \\
\text { higher education learning } \\
\text { o Demonstrate to top } \\
\text { management the potential } \\
\text { benefits ICT can bring to } \\
\text { higher education teaching } \\
\text { and learning }\end{array}$ \\
\hline
\end{tabular}

Table 14: Organisation context factors 


\section{Finance}

The findings showed that lack of finance was an important issue at the higher education institutes (refer section 6.4.1). Most institutes lacked the financial support ICT adoption, due to limited budget allocations. The data showed the high costs of running the institutes and acquiring appropriate infrastructures for students and teachers. Government and higher education institutes within SIDS such as the Maldives should explore ways to obtain finance. The required funds could be potentially provided by international donor organisations or acquired from Government projects.

\section{Centralisation}

The research data showed that centralisation of higher education services was found to be a challenging and complex issue. The findings highlighted how centralisation was hindering the higher education services, and social and cultural aspects. For example the students from the islands had difficulty attending higher education, because all the institutes were based in Male'. The literature confirmed that the Maldives is a country with a centralised system of Government administration and Male' is where the main business is concentrated (Adam \& Urquhart, 2007; Aneh Dhivehi Rajje, 2009-2013), although decentralisation was a key priority for the new government (Country Report, 2009).

The research data showed that the population of the Maldives was distributed among the outer atolls, and there had always been a tendency for migration to Male', for better higher education and employment opportunities. The centralised system of the Maldives (Adam \& Urquhart, 2007; Country Report, 2009) with its poorly developed inter-island transportation system was a major concern. Due to the existing transportation system, the Government was developing a national transportation system as a means to overcome the transportation barrier in selected regional hubs. The presence of such facilities should serve as an incentive for the population to coalesce around these regional hubs rather than in Male'.

The most practical possible solution would be to decentralise the higher education services to the remote islands. The research participants expressed that 
centralisation issues should be addressed by decentralisation. However, decentralisation was hampered by lack of infrastructure and the poor inter-islands transport system (Ibrahim, 2006). Nevertheless, this could be achieved through the provision of ICT services to the remote islands. Therefore, the Government and higher education institutes should support decentralisation of higher education at atoll and island levels.

This research did not investigate how many females and males were deprived of higher education on the remote islands. There were no exact statistics available nationally, but there would be many students who could not come to Male' for higher studies. The data evidence showed that ICT could play a role in delivering higher education services to the remote islands of the Maldives. Based on the evidence from the data, the researcher believes better utilisation of existing ICT infrastructures and integration of ICT into higher education is crucial. At institutional and national levels, the Government should ensure policy and regulatory frameworks are available to support the uptake of ICT in higher education.

In conclusion, it was found that centralisation is an important factor influencing the adoption of higher education in SIDS in general.

\section{Human Resources}

The research confirmed that there was a crucial need to develop skilled ICT human resources. Due to the small population, there were insufficient human resources to cater for the entire population. With the increase of new technologies there was a demand to increase the professional teacher capacity, ICT-skilled technicians, and academic managers, in order to embrace new technology in the learning and teaching process. According to the data evidence from the interviewees and focus group sessions, this factor was seen as a crucial element in order to deploy higher education in the remote islands.

The study has confirmed the findings of Kozma et al. (2004) and Aduwa-Ogiegbaen and Iyamu (2005), that lack of ICT skills is a major impediment to successful 
adoption of ICT into higher education. Adam and Urquhart's (2007) research paper also showed that in the case of the Maldives skilled human resources were critically required. The findings regarding the lack of human resources in the Maldives revealed the same weakness pointed out by Binger et al. (2004) as existing in SIDS.

The findings also confirmed that the Maldives does not have enough human resources to cater for the country's requirements (Adam \& Urquhart, 2007). The primary and secondary education sectors depended on expatriate teachers due to the unavailability of locally trained teachers. However, the higher education sector was less dependent on foreign teachers due to the availability of local teachers.

The Maldives relies heavily on foreign workers (Ibrahim, 2010). Technical human resource development in the Maldives requires capacity building, training, and employment opportunities in ICT. In light of these critical challenges, the need for higher education is important, particularly for the youth unemployed due to lack of qualifications or human capital with appropriate programmes for youth.

The youth population was high and there was growing concern about unemployment despite remarkable economic growth during the past three decades (MHYRS, 2010). There was a shortage of ICT skilled people, not only in tertiary institutes but also in the Government agencies. This issue is already posing even greater challenges and its severity continues into the future. Several ICTs, such as smart boards, multimedia technologies, computers and i-phones, were underutilised due to lack of skilled personnel. Technical support to maintain the resources and a significant level of skills was needed to take full advantage of the potential of ICT to transform learning (Bingimlas, 2009).

About 30 years ago the Maldives launched a basic literacy programme, the outcome of which was very successful (Department of National Planning, 2011). The Maldives reached an almost 100\% literacy rate (Department of National Planning, 2011; UNICEF, 2010). According to UNESCAP (2008), the Maldives had one of the highest adult literacy rates in the Asia and Pacific Region. However, 
these statistics did not indicate whether people were highly qualified or had the required ICT skills.

A long history of using traditional methods of teaching had failed to produce skilled ICT workforces nationally or within the higher education sector. Over the last years, less emphasis had been given by the Government to train people in acquiring ICT skills. It was hard to find people with the skill sets required to operate the facilities (Ibrahim, 2006). ICT-skilled people should be deployed on the islands for the teaching and learning process. However, ICT-skilled people could only be attracted to the islands by better incentives.

In conclusion, with regard to ICT skills, the research findings indicated that awareness programmes could plays a vital role in the ICT adoption process at national level. Lack of skilled ICT personnel remained a major impediment and researcher concludes that providing skilled people is a critical factor in the adoption of higher education. Therefore, ICT skilled people should be trained and encouraged to make themselves available in the remote islands to cater for the increased demand within the higher education sector.

\section{Top Management Support}

The findings showed that top management support would be a critical component in the ICT adoption in the higher education. The top management should encourage changing the traditional management procedures. The research showed that changing the mind-set of top management would be difficult and resistance encountered. Also, ICT adoption in higher education required top management support, which played a central role in the process.

Top management appeared to regard the traditional methods of teaching and learning as more beneficial that the technology view. Top management resisted using technology due to lack of ICT awareness and the potential benefits of ICT were unknown. In addition, top management resisted due to the fear of job loss and authority. Motivation and awareness were two points highlighted from the findings. In order to motivate and create awareness, the top management need to 
be made aware of the potential benefits ICT could bring to higher education teaching and learning.

\subsubsection{Environment Context Discussion}

The environment context plays an important role in the model in terms of understanding about social issues, cultural context and pedagogical issues (see table 15).

\begin{tabular}{|c|c|c|}
\hline $\begin{array}{l}\text { THEMES FROM } \\
\text { LITERATURE } \\
\text { REVIEW }\end{array}$ & $\begin{array}{c}\text { FINDINGS IN THIS } \\
\text { RESEARCH }\end{array}$ & RECOMMENDATIONS \\
\hline $\begin{array}{l}\text { Social issues } \\
\text { o Social Aspect (refer } \\
\text { section 2.9.1) }\end{array}$ & $\begin{array}{l}\text { Social issues } \\
\text { o Overcrowding in Male' } \\
\text { (refer section 6.5.1.1) }\end{array}$ & $\begin{array}{l}\text { Social issues } \\
\text { o Government policies should } \\
\text { include decentralisation of } \\
\text { services } \\
\text { o Government and higher } \\
\text { education institutes should } \\
\text { ensure to incorporate many } \\
\text { secondary school leavers to } \\
\text { higher education studies. }\end{array}$ \\
\hline $\begin{array}{l}\text { Culture issues } \\
\text { o Culture (refer } \\
\text { section } 4.4 \text { ) }\end{array}$ & $\begin{array}{l}\text { o Cultural homogeneity } \\
\text { among students and } \\
\text { teachers (refer section } \\
6.5 .2 .2 \text { ) } \\
\text { o No culture of using ICT } \\
\text { in academic learning } \\
\text { (refer section } 6.5 .2 .3 \text { ) }\end{array}$ & $\begin{array}{l}\text { Culture issues } \\
\text { O Island female students should } \\
\text { have more opportunities for } \\
\text { higher education learning } \\
\text { o Gender gap in education } \\
\text { should be narrowed } \\
\text { o Cultural homogeneity should } \\
\text { support the ICT adoption in } \\
\text { higher education } \\
\text { o Limited culture of using ICT in } \\
\text { academic learning should be } \\
\text { addressed in order to foster } \\
\text { greater technology utilisation } \\
\text { in higher education }\end{array}$ \\
\hline
\end{tabular}




\begin{tabular}{|l|l|l|}
\hline $\begin{array}{c}\text { THEMES FROM } \\
\text { LITERATURE } \\
\text { REVIEW }\end{array}$ & $\begin{array}{c}\text { FINDINGS IN THIS } \\
\text { RESEARCH }\end{array}$ & \multicolumn{1}{c|}{ RECOMMENDATIONS } \\
\hline $\begin{array}{l}\text { Teaching and } \\
\text { learning (Pedagogy } \\
\text { practices) }\end{array}$ & $\begin{array}{l}\text { Teaching and learning } \\
\text { O Pedagogy (refer } \\
\text { section 2.9.2) }\end{array}$ & $\begin{array}{l}\text { Teaching and learning } \\
\text { (Pedagogy practices) }\end{array}$ \\
& section 6.5.3.1) & $\begin{array}{l}\text { O Culture of spoon-feeding } \\
\text { teaching styles should be } \\
\text { replaced my more student- } \\
\text { centred approach. }\end{array}$ \\
\hline
\end{tabular}

Table 15: Environment Context Factors

\section{Social Issues}

The findings in this study highlighted overcrowding in Male' was the result of centralisation, which led to social issues. The regional development and population consolidation process started in the mid-1990s to maximise economies of scale in the provision of socio-economic services. This process was started to support the inhabitants of remote islands to voluntarily move to larger islands, where socio-economic services and employment opportunities were available to improve the standard of living.

The issue of overcrowding in Male' was raised by the research participants as an issue of great concern. The high influxes of expatriate workers had created local youth unemployment and social problems. The locals were not willing to take up jobs because of low skills or wages. There were no unemployment benefits and some of these youths hung out in the streets. Due to overcrowding in many capital islands of the Maldives, the research revealed several social issues such as drugs and gang violence. Research participants noted the increased use of drugs among youths, which extended to all families because of the threat posed by drug abuse to society and the image of the Maldives. Literature also confirmed drug abuse had become a serious lifestyle issue among affected youth (Ageel, 2006; FASHAN \& NCB, 2003).

The researcher concludes that social issues were important factors to address and ICT could alleviate these problems by providing higher education opportunities, 
particularly for students living in the remote islands. Therefore, the Government policies should foster the decentralisation of higher education services.

The research revealed that the increase in social problems arose due to lack of opportunities for higher education and employment. The literature also showed that the increased number of youths as well as unemployed was due to the massive number of yearly school leavers (Narcotics Control Bureau, 2006).

Due to the limited higher education opportunities, students were involved in gang violence and theft and also exposed to drugs. Information and Communications Technology could have a role, as the UNESCO (2005) report stated: "ICT can also play a major role for the diffusion of information on matters having direct impact on the social, cultural and economic life of the youth" (p.54). In summary, the Government and higher education institutes should seek ways to encourage more secondary school leavers to engage in higher education studies.

\section{Cultural issues}

\section{Gender disparity in higher education}

The research has shown evidence that cultural disparities existed between males and females in terms of higher education opportunities. Literature (UNESCO, 2011) has shown that in the Pacific Islands women benefit through distance learning. Looker's (2008) research showed that cultural issues with respect to appropriate gender behaviour and attitudes were particularly important in the developing world. Efforts were needed among many stakeholders, such as Government, private sector and ICT companies, to facilitate job placement opportunities for ICT females (Elnaggar, 2008).

This research also confirmed that, with respect to the Maldives' culture, children (i.e. male or female) below 18 years were not encouraged to live alone. Even when the children reached above 18 years of age or older, parents provided all the required support. This feature is different compared to Western countries. The research participants expressed that the parents followed a strict pattern with 
regard to girls in the Maldives. Most females lived with their parents even if they were married and it was similar for most males as well.

Literature has shown that this kind of cultural aspect occurs in in Arab countries. As an example, Elnaggar (2008) explained that female students in the higher education institutes of Saudi Arabia, UAE, and Oman were not allowed to leave the campus without their families' permission. Culturally, in the Maldives parents take care of the children even when they are adults.

Digital divide is seen in terms of the ways females are disadvantaged relative to males (Looker, 2008). Female students do have cultural restrictions imposed by the parents and caretakers. Nonetheless, the gender gap in education is closing since there are equal opportunities for girls and boys in schools. There are wide differences within and across countries and far too many females experience disadvantages (Elnaggar, 2008; Looker, 2008) or leave prematurely from school. With regard to social and cultural reforms, the Maldives is quite a number of years away from treating women on same footing as men (Henderson, 2008).

This research did not find any evidence of how many females and males are deprived of higher education on the remote islands. In addition, there are no exact statistics available nationally, there will be many students who cannot come to Male' for higher studies.

In conclusion, the researcher believes that the culture aspect is important point to consider. This research did not focus on the exact gender divide in the utilisation of ICT in the Maldives, which would be another topic of research on its own. Nevertheless, providing greater higher education opportunities for female students on the remote islands would help narrow the gender gap in education.

\section{Cultural Homogeneity among students and teachers}

The findings in this research have revealed the cultural homogeneity among students and teachers. Cultural homogeneity meant that everyone knew each other and saw each other very often with little cultural diversity. 
The islands are small with people living close to each other in small communities. In daily life, they interact with each other often. The Maldivian society is a close society where everyone knows each other. There are friends everywhere with whom living in a harmonious society with no secrets. A special value of living together is a basic part of our unifying culture. The social harmony shows the result of the education system that teaches our young people to live together. In conclusion, this cultural homogeneity should operate to support ICT adoption in higher education.

\section{Limited culture of using ICT in academic learning}

The findings in this research also revealed that there was limited culture of using ICT within the higher education sector despite its adoption for social purposes. The reviewed literature about culture in this study (refer section 2.9.1) also confirmed that cultural perceptions towards computer related technologies existed. Myers and Tan (2002) highlighted the role of culture in implementing ICT deployment. Literature from Somekh (2008) confirmed that culture, social and organisation contexts directly influenced ICT integration in teaching and learning.

The limited culture of using ICT for academic learning in general resulted in a lack of technology utilisation within higher education. In concluding, creating a culture of utilising ICT in academic learning is important and should be taken seriously. This research has clearly shown that students were utilising ICT for social communication activities but not necessarily for academic activities.

\section{Teaching and learning (Pedagogy practices)}

The research data showed that the teacher-centred methods through direct explanation and some discussion were the primary methods employed by teachers in primary, secondary and tertiary education. The research confirmed that the traditional method of delivery was practised throughout the education system, which was inconsistent with the introduction of technology in tertiary education.

In the teacher-centred approach, teachers are the primary source for knowledge, which leads to rote learning (i.e. dictating and learning teacher notes or lectures) 
as was the norm in the Maldives. In contrast, the student-centred approach focuses on students' needs, abilities and interests, with the teacher as a mentor.

The literature showed the broader requirements of pedagogical practices. Effective pedagogical integration required a change process where all stakeholders were involved to re-examine their existing structures (Sife et al., 2007). Bates (2000a) suggested that minor adjustments in current practices would be required if tertiary institutes were to successfully integrate technologies for teaching and learning. The pedagogy of ICT should be explained within a broad framework of educational practice (Webb \& Cox, 2004) to decision makers at Ministerial level in order to enable the practice to become universal in the higher education system. This initiative should come from academic managers within the tertiary institutes.

The findings of the researcher confirmed that the teacher-centred approach dominated the existing education system. This study showed that the traditional practices needed to change. As literature from Douglas (2011) confirmed, ICT utilisation in teaching and learning is based more on a learner-centred approach. The data revealed teacher resistance to technology, since it required different pedagogical practices. $\mathrm{Hu}$ and Webb (2009) highlighted that the teachers were often aware of the benefit of ICT, but implementation of the pedagogy was "alien".

Consistent with the literature (Bates, 2000a; Douglas, 2011; Guttman, 2003; Valentine \& Holloway, 1999; Webb \& Cox, 2004), the student-centred approach is a method of education where the main focus is on the students instead of the teachers or lecturers. This method puts the students first. In order to make the student-centred approach a success, there would be many implications for the design of curriculum and course content (Oliver, 2002).

In essence, several researchers (Bates, 2000a; Jones \& Cresse, 2001; Kozma et al., 2004; Tinio, 2000) believed teaching and learning was moving to a technologybased concept. This would require a paradigm shift from teacher-centred to constructivist-learning paradigm (i.e. student-centred approach) and the researcher believes that this paradigm shift could occur based on the recognition 
of learning styles appropriate for the technological world. The technology-based higher education system would likely be closer to the student-centred approach.

In concluding, social, culture and pedagogical practices are important factors to consider when it comes to ICT adoption in higher education of SIDS. Furthermore, a more student-centred approach should be implemented, thus creating less of a culture of spoon-feeding.

\subsubsection{Geography Context Discussion}

The geography in the Maldives poses logistical, social and cultural problems. The analysed data in this research reinforced the connection between the geography and the process of ICT adoption (see table 16).

The geography factors identified in the proposed framework are relevant and applicable. There were many students who had completed the pre-university requirements but were deprived of higher education, due to geographical problems. Therefore, the researcher believes geography is the most important barrier in the adoption of higher education and ICT could offers tools to overcome the geographic barrier.

\begin{tabular}{|c|c|c|}
\hline $\begin{array}{c}\text { THEMES FROM } \\
\text { LITERATURE REVIEW }\end{array}$ & FINDINGS IN THIS RESEARCH & RECOMMENDATIONS \\
\hline Geography & Geography & Geography Context \\
\hline $\begin{array}{l}\text { o Geography (refer } \\
\text { section } 4.2 \text { ) }\end{array}$ & $\begin{array}{l}\text { o Geography context in the } \\
\text { Maldives (refer section } \\
6.6 .1 \& 6.6 .2 \text { ) }\end{array}$ & $\begin{array}{l}\text { o Bridging the } \\
\text { geography separation } \\
\text { through ICT by the } \\
\text { Government }\end{array}$ \\
\hline $\begin{array}{l}\text { o Transportation } \\
\text { (refer section } 4.2 .3 \text { ) }\end{array}$ & $\begin{array}{l}\text { o Transportation (refer } \\
\text { section 6.6.3) }\end{array}$ & $\begin{array}{l}\text { o Complete } \\
\text { transportation system } \\
\text { should be established } \\
\text { to travel between } \\
\text { islands }\end{array}$ \\
\hline
\end{tabular}




\begin{tabular}{|c|c|c|}
\hline $\begin{array}{c}\text { THEMES FROM } \\
\text { LITERATURE REVIEW }\end{array}$ & FINDINGS IN THIS RESEARCH & RECOMMENDATIONS \\
\hline & $\begin{array}{l}\text { Taking higher education } \\
\text { to remote islands } \\
\text { o Taking higher education } \\
\text { services to remote islands }\end{array}$ & $\begin{array}{l}\text { Taking higher } \\
\text { education to remote } \\
\text { islands } \\
\text { o Higher education } \\
\text { should be taken to the } \\
\text { islands through ICT. } \\
\text { o ICT should be seen as } \\
\text { the ultimate solution } \\
\text { to bridge the higher } \\
\text { education divide. }\end{array}$ \\
\hline
\end{tabular}

Table 16: Geography context factors

\section{Geography}

This research showed the geography of the Maldives caused problems in delivering higher education services to distant islands. As several participants pointed out one of the greatest challenges for the Maldives was that the population was dispersed across many islands. This exact point was raised by Lane (2009), that geography is a barrier for delivering higher education. Several studies (Koonjul, 2004; Shareef, 2005; Shareef \& Kinshuk, 2002; Shareef \& Kinshuk, 2006; WHO, 2006) highlighted that small populated islands experienced diseconomies of scale, which led to difficulties in delivering higher education services to the inhabited islands.

The researcher believes that geography is the most critical factor to consider when it comes to SIDS like the Maldives. Due to the topography of the islands, the conventional form of higher education delivered primarily at higher education institutes was impossible and this is where ICT could have a role to play. The Government must play a vital role in bridging geographic separation through the use of ICT.

The research confirmed that, given the geographical problems, it would be difficult to set up higher education institutes on each inhabited island due to limited 
resources and poor transportation systems. All basic education services were available on all inhabited islands but the services differed greatly in their quality in comparison to what was being offered in Male'. Likewise, a complete transportation system should be established to facilitate travel between islands.

The researcher expected that the geography would be raised by the research participants as a focus of discussion when talking about integrating ICT into higher education. This is fact that can never be ignored in the context of the Maldives.

The research participants expressed that geography was one of the important factors in the context of the Maldives. This study also confirmed the centrality of geographical difficulties to the subject of the research. Literature shows that there are severe challenges faced with respect to remoteness of islands (Country Report, 2009; Lane, 2009; Shareef et al., 2010).

The Maldives is one of the few countries among the 52 SIDS where there is a multitude of remotely located inhabited islands (UNCTAD, 2004b). This made it challenging to distribute the general higher education services to these remote islands. Remoteness of rural areas counted as a barrier (Lane, 2009).

The country's numerous small island communities (Hussain, 2008; Shareef et al., 2010) were highlighted by the participants of this research. The participants expressed that the use of technology (i.e. ICT) to communicate between remote islands would be the ultimate solution to address the geographical difficulties. The remoteness could be greatly reduced with wide usage of ICT resources, which were available both in Male' and rural areas for teaching and learning. Therefore, ICT expansion can virtually reduce the geographical separation and take advantage of the education facilities available in other islands particularly in Male' (InfoDev, 2010a). 


\section{Taking higher education to remote islands}

The literature has shown the concept of higher education divide (Athif \& Pimenidis, 2009; Lane, 2009). The research participants noted that higher education services were primarily available in Male' and there was a general lack of higher education within the remote atolls.

The urgency to apply ICT in different facets of higher education, economic, social and other aspects would be vital to allow higher service delivery to these remote areas. The research findings showed that participants raised serious concerns regarding the delivery of higher education services to the remote islanders. The Government was facing the challenge of providing higher education to all inhabited islands, which had now become both a necessity. However, the need to take higher education services to the islands through ICT should be prioritised by the Government.

The findings also reflected that the major reasons for in-migration were the higher standards ICT would allow student to utilise the existing resources while living on their islands. Students remaining on the islands would lead to a reduction in the influx migration of people to Male'.

The findings showed that costs of higher education were high due to the extremely high living standards in Male'. There were no available statistics to confirm how much parents were spending on the higher education of students. Nevertheless, the researcher understands, parents from the islands had to spend a lot of money to pursue higher education for their children in Male'.

Most participants stressed the urgency for taking higher education to the islands through ICT. The participants were generally aware of the challenges associated with taking ICT-based higher education to the remote islands but appeared to believe these were no worse than the lack of opportunities when students had to attend Male' for higher studies. 
In conclusion, the researcher believes that providing higher education at island level by using ICT would be the ultimate solution to bridge the higher education divide.

\subsection{Practical roadmap, grounding UNESCO's Continuum Model of ICT development with TOEG framework}

The UNESCO model has four broad stages of development in terms of adoption and use of ICT (UNESCO, 2002). They are emerging, applying, infusing, and transforming (Olakulehin, 2007; UNESCO, 2002). Grounding the factors discovered in this study within UNESCO's continuum model of ICT development provides a gateway to adoption of ICT within higher education. This plan could also make it possible to apply the existing technologies and provide a framework for technology adoption within higher education.

Figure 25 shows the continuum model where four major steps (emerging, applying, infusing and transforming) are considered. The indisputable importance of ICT would be a major driver of Maldivian higher education as a critical tool for better higher education, connecting island students and providing choice for most learners. The specific themes that emerged from this research are applied. 


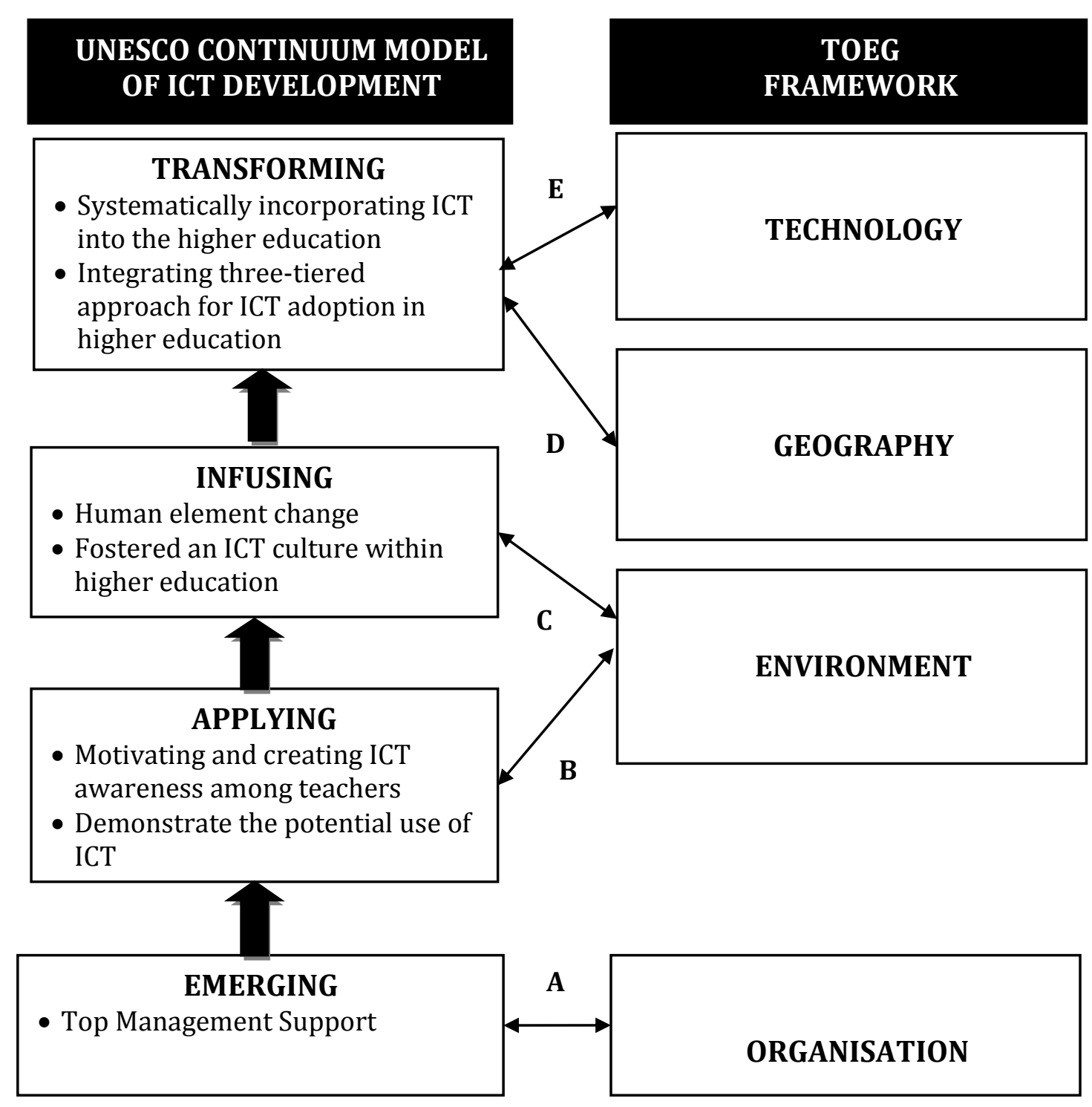

Figure 25: Practical roadmap by grounding UNESCO's continuum for ICT adoption with TOEG framework (adapted from: UNESCO, 2002)

\subsubsection{Emerging}

Link A - Organisation (TOEG) with Emerging (UNESCO Model): There is a link between organisation (i.e. TOEG) to the Emerging stage of the UNESCO continuum mode of ICT development (Link A). Top management support is vital for ICT adoption in higher education. The Emerging stage is where higher institutes are in the early stage of utilising ICT for teaching and learning. Top management are in the beginning phase of using ICT. This phase is typically rooted in spoon-feeding pedagogical practices. 


\subsubsection{Top Management Support}

A significant number of research participants expressed concern about the need for top management support. Emerging is the initial step of ICT development and lack of top management in organisation support might be because top managers did not understand the expected benefits of ICT within higher education. The literature has shown that there was a general lack of knowledge (Bingimlas, 2009; Demiraslan \& Usluel, 2008) and ICT skills on the part of teachers and academic management.

\subsubsection{Applying}

Link B - Environment (TOEG) with Applying (UNESCO Model): Environment (TOEG framework) has a relationship with the Applying stage of the UNESCO model where teachers are using ICT in their classrooms and ICT can be integrated into the curriculum. Creating ICT awareness among teachers about the potential for utilising ICT by demonstrating the benefits is needed at this stage.

\subsubsection{Motivating and creating ICT awareness among teachers}

The data showed that teachers' negative attitudes towards technology were a barrier to the adoption of ICT to higher education system. The reviewed literature confirmed this concept, as Sife et al.'s (2007) research agreed that full support of all academics, students and teachers should be a key feature in addressing the awareness and attitude problems. Khan et al. (2012) agreed that teachers' positive attitude is necessary for ICT adoption.

Mind-set and motivational change about ICT was required among teachers and top academic managers, who would need to be convinced to incorporate ICT within teaching and learning. Teacher motivation was become an important issue in technology integration into the higher education system. The findings showed that the teachers tended to resist any new technology use in higher education. Creating awareness among teachers would now be essential in order to make things happen.

Positive attitudes of teachers towards ICT could help the uptake of ICT in professional development programmes. These programmes could be customised 
to the specific needs of teachers and would create awareness for teachers to use new technologies. The more professionally capable the teachers were in ICT, the more chances of using ICT in the classrooms in teaching and learning.

Reddi and Sinha (2005) pointed out that one of the major constraints to the use of ICT in the Maldives at classroom level was the lack of awareness and basic ICT skills or competencies. At present access to higher education opportunities were denied to the great majority of the population mostly due to the limited presence of higher education service providers and also the high cost of higher education abroad (Country Report, 2009).

The need for nationwide IT awareness and development of IT skills was highlighted in the literature (Adam \& Urquhart, 2007). The awareness programmes could be an on-going process. As Bingimlas (2009) stated, "teacher training in the use of modern technology in the classroom helps to increase the teachers' efficiency in using ICT in education effectively" (p.242). ICT awareness could be created in students, teachers, academic management and parents by higher education institutes.

Formally organised awareness programmes could be conducted in the Male' and in the remote islands. Short seminars could contribute to raising the awareness and changing the attitude of teachers and academic managers towards ICT facilities and services.

Parents are important people to create the necessary awareness. The interpretation of the findings showed that parents played an important role in students' education. Parents should be aware of the benefits ICT will bring to the higher education system. The awareness programmes should be conducted at island levels and atoll levels. The Ministry of Education and tertiary institutes should take the initiative in providing the necessary information to impart ICT knowledge. 
In conclusion, motivation and awareness among teachers would be an important step to ensure that they understood the perceived benefits ICT could provide when used in higher education.

\subsubsection{Demonstrate the potential use of ICT}

The research findings emphasised that demonstrating ICT use to students, teachers and academic management was an important element. With modern development, ICT know-how is important for higher education institutions, Government, students, teachers, parents and academic management. There was a general lack of understanding about ICT and no demonstration of the expected benefits ICT could bring to their teaching and learning.

Relative advantage from Rogers' characteristics of adoption of innovation is relevant. The students and teachers needed to perceive the significant objective advantage of the adoption of ICT in higher education. This characteristic would be important, as ICT use must be perceived as an advantage by higher education personnel (students, parents, administrators, and decision-makers) in order to expand the adoption rate.

The academics need ICT know-how in order to contribute to bridging the digital divide between atolls. This might entail promoting digital inclusion by exploring and demonstrating how ICT could be used in higher education to reach the islanders.

The traditional methods were more familiar to teachers, so a clear demonstration of the anticipated benefits of ICT would be an important step in harnessing higher education. The teachers were reluctant to utilise ICT in the classrooms due to their lack of ICT knowledge compared to students. Teachers needed to be encouraged to reflect on and make their own decisions about ICT needs with good demonstration facilities, on an on-going basis. This would allow more involvement and greater integration of ICT within the teaching and learning process. 
Therefore, demonstrating the benefits would be equally important to conceptualise the benefits of ICT to higher education.

\subsubsection{Infusing}

Link C - Environment (TOEG) with Infusing (UNESCO Model): The Infusing stage (UNESCO) is where ICT is integrated across the curriculum. Environment (TOEG framework) has a direct influence on the Infusing stage (UNESCO model), fostering ICT culture in higher education and changing human elements. In addition, ICT is utilised in other areas such as for administrative purposes. Teachers can use ICT to explore new ways to solve everyday problems and real world situations.

\subsubsection{Human element change}

The research has confirmed that for ICT to be integrated into the mainstream of higher education will need a changing of mind-set. A shift in mind-set is important when change is required to happen among top management and teachers. In contrast, it is almost certain that the students who enter the tertiary institutes today are younger than the microcomputer, and are more comfortable working on a keyboard than writing in a notebook. This mind-set change could happen if ICT were introduced in the primary and secondary schooling systems and at the grass roots level of teaching.

A pedagogical change in teaching and learning practice is required, away from the traditional style to the technology-driven style. A more technology-friendly environment for students and teachers to foster less traditional methods of teaching is required. This change could be brought about by careful planning and a mind-set change at all levels. The cultural factors should also be considered. A good example would be teachers being prepared to experiment with ICT in their classroom delivery despite the perceived risk to their status.

Information and Communication Technology utilisation should also continue within secondary level teaching and up to higher education levels. The number of students finishing secondary education (i.e. Grade 10 or 12 schooling) who are 
seeking entry to higher education is also increasing. Innovative ways in expanding access and providing more equitable opportunities for those remote islands should be considered.

To address the issues of higher education, a solid foundation is required by using ICT at grass roots (i.e. primary and secondary education) levels. In the Maldives, ICT was still being seen as an add-on within the teaching system and many teachers were still concerned with "teaching ICT" rather than "teaching with ICT".

According to the Maldives National Development Plan, there should be more educational opportunities for the Maldivian people through introducing important Government policies, and widely enhancing knowledge, both in urban and rural communities (Department of National Planning, 2011). The higher educational opportunities should reduce differences between the knowledge levels of the populations on urban and rural islands. The use of ICT could provide chances for remote learners and build a knowledge-based society, which could enhance the nation's competitiveness.

In conclusion, human element change is required in order to change the traditional methods and adapt to new methods.

\subsubsection{Foster an ICT culture within higher education}

ICT is regarded as an important subject by the national education curriculum of the Maldives. Studying cultural perceptions was particularly important in the context of developing countries where ICT was not usually part of their culture (Albirini, 2006). This technological aspect certainly had a place in higher education institutes, and would simplify and improve primary activities.

Albirini (2006) expressed that ICT might have cultural influences in developing countries due to its novel presence in society and at institutes. An ICT-based culture could be created by increasing the capability and independence to search for materials and knowledge within the teaching and learning process. 
In conclusion, creating a holistic culture of utilising ICT within higher education is vital.

\subsubsection{Transforming}

Link D - Geography (TOEG) with Transforming (UNESCO Model): Transforming (UNESCO model) is the final stage where there is a shift from the teacher-centred approach to a student-centred approach. Geography (TOEG framework) has a direct influence on the Transforming stage (UNESCO model) using the three tiered (i.e. urban, atoll and island) approach to completely integrate ICT into all higher education teaching and learning. Both teachers and students can utilise ICT in teaching and learning.

Link E - Technology (TOEG) with Transforming (UNESCO Model): Geography (TOEG framework) has a direct influence on the Transforming stage (UNESCO model) where ICT is systematically incorporated into the higher education system. Finally, ICT is completely integrated into all higher education teaching and learning. Both teachers and students can utilise ICT in teaching and learning.

\subsubsection{Systematically incorporating ICT into the higher education}

Through the voices of participants, the research findings confirmed that information technology was not incorporated into the school system but instead taught as a subject in some primary and secondary schools. A common wish from the interview and focus groups participants in this study was to introduce ICT into the education system of the Maldives. A systematic plan would be necessary for ICT integration into the education system. Sife et al. (2007) noted that many tertiary institutes in developing countries had embraced the ICT integration process without clear plans.

ICT is simply a set of very powerful tools that enable students to learn other things. ICT as a tool can contribute to higher education and should be introduced into school strategic plans and teaching plans (Sangrà \& González-Sanmamed, 2010). 
A step-by-step approach would be ideal to ensure ICT incorporation is practical. The focus on using information technology should be increased by allowing primary school children to use ICT. Interactive participation is important when ICT is deployed at school levels. The first step would be to create awareness programmes about ICT technologies among teachers and academic management. The Ministry of Education is now trying to incorporate ICT into the mainstream education of teaching and learning.

Systematic integration of ICT into the school system would be a vital ingredient for the successful outcome of ICT integration into the higher education sector. This initiative would certainly create more technology-capable students, thus increasing the likelihood of getting more ICT skilled students in the tertiary education sector.

In conclusion, systematic incorporation of ICT at school is required in order to create technology savvy academic management and students. 
8.4.4.2 Integrating three-tiered approach for ICT adoption in higher education teaching and learning in SIDS

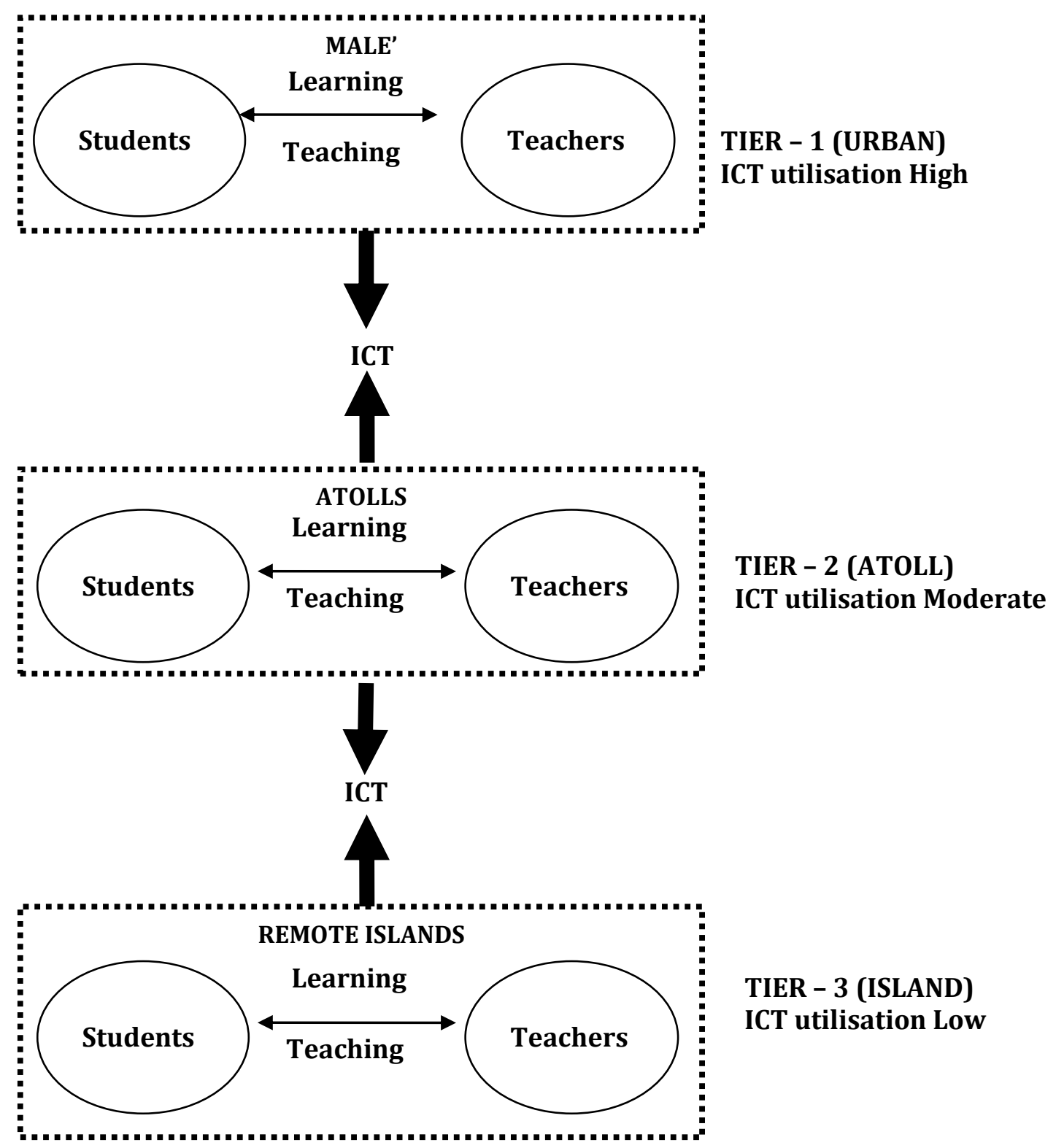

Figure 26: Three-tiered approach for ICT use

The adoption of ICT in the higher education sector would be practical with a threetiered approach. Figure 26 shows a three-tier architecture portraying the student, the teacher, and the link between the two. The three-tiered framework comprises three major tiers: Male', Atolls, and remote islands. The three tiers differ in terms of ICT utilisation (Tier 1: high; Tier 2: moderate; and Tier 3: low). 
It seems that the delivery of higher education services to the remote island level through online services should now become a mandatory requirement with the Maldives' unique geographical diversity. Islanders (i.e. learners and teachers) cannot come to Male' for higher education and development of separate physical higher education institutions would not be cost-effective in the atolls. With the existing technological infrastructures, it should be possible to setup a mechanism where islanders can learn while staying on their respective islands.

\section{Link between Male' to Atolls}

The central hub for all activities would be Male' and the Capital Islands of the atolls would be regarded as sub-hubs. The proposed three-tier model presumes that the students and teachers in Male' have reliable Internet connections and can go online anytime on their own computers or have access to computers within the higher education institute.

All communications between the atoll capital islands and Male' could happen in batch mode (i.e. not requiring continuous online connection) or online mode (i.e. continuous online connection) if necessary. The batch mode would be suitable for islands with less Internet connectivity and the online mode for the capital atoll islands where Internet connectivity is high.

\section{Link between Atolls to Remote Islands}

The proposed three-tier framework acknowledges that the students and teachers on a few remote islands might not have reliable Internet connections or be able to go online anytime with their own computers or computers in a higher education institute. Despite this fact, most islands do have Internet connections, which would offer a realistic possible option for students and teachers to go online.

The remote islands would be treated as sub-hubs under the atoll level. With the existing setup the remote islands do not have TRCs to support higher education teaching and learning. The remote islanders could travel to the capital islands where the TRCs are based. The remote islanders could also use mobile connections, which are available throughout the country. 


\section{Asynchronous Learning vs Synchronous Learning}

Asynchronous learning is close to constructivist theory since it focuses on a student-centred approach. The remote islanders could use Learning Management Systems (LMS) such as WebCT or Moodle. When the students from remote islands go to the atoll islands, the synchronous forms of communication could be supplemented with the inclusion of video, audio, video conferencing and Skype. The social constructivist pedagogy fits with the concepts such as discussion forums, blogs, wikis and on-line collaborative activities.

The students could be physically present in classrooms at the atoll and Male' levels. Synchronous learning can take place where students are exposed to the same content, at the same time and same place. A lecture undertaken in Male' could be in face-to-face mode and a teacher could use video conferencing tools to deliver the lecture to atoll level students. The same lecture could be viewed at the remote island level or downloaded for later viewing if the Internet connection were good.

\section{Virtual Island Higher Education Concept - VIHE}

The VIHE concept could provide students with instructions from teachers in an interactive environment. A qualified teacher could be stationed in Male', where students would have direct access to instant feedback. The VIHE concept could also provide a structured schedule of classes, which would be helpful and provide students with the freedom of asynchronous learning.

The spectrum of teaching modes in VIHE includes courses based on video, audio, video conferencing, and WebCT and Blackboard could also be used. The VIHE concept would be helpful for those students who were keen to undertake their tertiary education on the islands. This concept would give students the opportunity to undertake learning activities $24 / 7$.

The goal of the VIHE concept would be to provide access to island populations unable to attend a physical campus in Male'. The concept of VIHE could also provide a social learning environment that closely replicated the traditional brick 
and mortar classrooms. The students who were unable to attend the virtual sessions could obtain a recorded version later.

\section{Perspective of using blended approach}

A blended approach (Alebaikan \& Troudi, 2010; Benson, Anderson \& Ooms, 2011) would combine face-to-face facilitation with computer-mediated instruction and mobile technologies. This could include educational technologies using computer, mobile devices, video conferencing and other electronic media. The perspective of blended learning provides more realistic practical opportunities for students and teachers to make learning independent, accessible, useful and sustainable.

The specific approach of blended learning used could be a combination of online and offline. The greater percentage should focus on offline delivery and less percentage on online in order to minimise the costs in the remote islands. This approach is a cost-effective model and might emerge as the predominant model of the future for countries with specific geographical problems.

ICT skills are critical if a blended approach is to be introduced on the remote islands. This would involve collaboration, resource sharing and content development, and learning could be undertaken digitally, asynchronously, and at a distance. It is unlikely or impossible that student learning will continue to be based solely on print textbooks and face-to-face classes with the given geographical context.

A blended approach would enable students to take part in both synchronous and asynchronous learning. The remote student learners could connect to the learning programme and to worlds outside the classroom. This would connect students and teachers more closely to the island communities, and significantly reduce the effects of remoteness.

The use of the traditional method showed that face-to-face contact is strongly valued in the Maldives. The interpersonal contact between students and teachers stems from pedagogical beliefs. Due to the existing situation in the Maldives, most 
institutes might not be technologically ready to move to a purely Internet-based method of instruction. The researcher believes, due to the existing traditional system, that the blended approach should have both face-to-face delivery for remote students and separate synchronous online classes.

\section{ICT tools}

Podcasting: Podcasting would be useful tool since it allows any student or teacher with a microphone, a computer and an Internet connection to communicate. The students on the islands could use podcasting to share audio broadcasts. The use of mobile technologies is common in the Maldives. Podcasts refers to a Real Simple Syndication (RSS) feed that could be downloaded so that they can be listened to. RSS is a feed that enables for the subscription of podcasts, blogs and web pages. Podcasting would provide several advantages to students, teachers and parents. As there are limited learning styles available at higher education institutes in the Maldives, students can use varying learning styles, aptitudes and remotely accessible sources of information.

Video Recorded Lectures: Video lectures would be good source for distributing materials of higher education learning to atoll and island levels. Recorded video lectures could be distributed through sea or air transportation systems or via ICT networks. The lecture recording would be carried out in a tertiary institute in Male', which has the recording facilities. The islands also have recording facilities, mostly at capital atoll islands. The video recording team would comprise a group of lecturers, technicians and other support staff. The recorded versions could be burned onto DVDs, which would be distributed to the islands.

Skype: Skype is a hybrid peer-to-peer system that makes it easy to run on computers. Skype would be a useful tool which could be used by the students and teachers in small groups. A dedicated synchronous teaching application could be used when dealing with large groups of students.

YouTube: YouTube is a simple website, which could be used by students and teachers, that provides educational videos at the click of a button. YouTube has the 
ability to broadcast to an audience of students and teachers who could be stationed anywhere in the Maldives or elsewhere.

\subsection{Adding value to research context among SIDS}

This study is contributing to the research conducted in SIDS and the Maldives. The new insights are provided by the model the nature of challenges facing the process of ICT integration within higher education in SIDS. The final results and conclusions drawn in this study are adding value to the lessons to be learned from SIDS such as the Maldives. The factors discovered can be transferred to the context of others SIDS since there are many similarities among these countries, including size, infrastructure, human resources, geography, digital divide, e-readiness and ICT policies.

The approach used in this study can also be followed by academic decision makers and top managers among SIDS. The use of the research findings is expected to help in portraying the uptake of ICT higher education. Many SIDS governments in the world can successfully use the outcome of this research to benchmark the strategies of higher education in utilising ICT.

The factors articulated in the findings can be transferred and redefined in SIDs contexts while the approach and methodology can be followed and adopted by policy makers in SIDS.

\subsection{Adding value to research context in the Maldives}

There is lack of lack of research and literature available in ICT adoption within higher education of SIDS. An understanding of the Maldives context is also not widespread in the literature. Therefore, this study is a noteworthy contribution to the literature. The new findings and analysis of the data add to the previously limited research conducted in this area.

The findings and data analysis that explained the factors make a further contribution to the limited understanding concerning the context of the Maldives. 
The uses of TOEG framework further adds value and covers four broad aspects: technology, organisation, environment and geography contexts.

\subsection{Chapter Summary}

This chapter presented a discussion of the key findings of this research. The study has provided a TOEG framework for SIDS such as the Maldives, based on the empirical data collected from the Maldives. This chapter strongly supported the theoretical viewpoint that context determines the use of ICT in higher education in SIDS such as the Maldives. The factors against the adoption of ICT outlined in Chapter 6 appeared to be of particular importance to this research.

The main categories of these factors are based on the technology, organisation, environment and geography contexts of the TOEG framework, combined with DOI theory and TAM theory. The identified factors in Chapter 6 together with the literature in Chapter 2 were compared to form the basis of this chapter. The factors discovered in chapter 6 have been validated and compared to the reviewed literature. The discussion confirmed the significance and of the factors in the context of the Maldives.

The second part of this chapter utilised the UNESCO's continuum model in grounding the factors and themes discovered in this research together with the TOEG framework. Four broad stages were applied to formulate a model in terms of adoption and use of ICT in higher education.

In the next chapter, the researcher will present the conclusions of this study and the main contributions to knowledge. The chapter will conclude by explaining the summary of key findings, limitations of the study, generalisation of findings to other SIDS, risks of the research, contributions of the research, conclusions drawn, research recommendations and suggestions for further research. 


\section{CHAPTER 9}

\section{CONCLUSION}

\subsection{Introduction}

This concluding chapter begins by providing a reminder of the two research questions raised in this study. The motivation for this research, limitations and associated risks are also documented in this chapter. The generalisability of the findings to other SIDS context, with the main contributions of this research and implications, are also discussed.

The chapter explains the research implications with respect to SIDS, within the specific context of the Maldives. The chapter concludes by providing recommendations for further research with final comments by the researcher.

\subsection{Motivation for this research}

This research emerged from the researcher's involvement with the higher education sector and use of ICT in the Maldives, a SIDS, over a period of fifteen years. The researcher took special interest in information technology and eCommerce as a career and worked in the higher education sector. The researcher was an active lecturer among the tertiary institutes in the Maldives, including The Maldives College of Higher Education and Villa College.

\subsection{Answering research questions}

This study sought to answer two main research questions:

Q1: What are the factors that influence the adoption of ICT within higher education teaching and learning in SIDS such as the Maldives?

This research question was answered by Chapter 6, which concluded that there were specific factors affecting the adoption of ICT in the higher education sector of SIDS such the Maldives. The factors discovered were crucial elements in understanding ICT adoption in higher education. 
Q2: How can ICT be used to address the issues within the higher education teaching and learning of the SIDS such as the Maldives?

A detailed solution of how ICT could be used to address the issues in higher education teaching and learning was discussed in Chapter 7. It concluded that a framework was necessary for ICT adoption in higher education in SIDS. A TOEG framework was developed based on the TOE framework. The revised TOE framework has four elements: technology, organisation, environment and geography.

Furthermore, the researcher further enhanced the framework by utilising components of two information system theories (TAM theory and DOI theory). The model was formulated by adapting the TAM to ICT use in higher education in SIDS (in the case of the Maldives).

\subsection{Summary of research findings}

The key findings of the research are based on the literature review reported in Chapters 2 and 4 and the data analysed and presented in Chapters 6 and 7 . The findings are summarised as follows:

1. The literature reviewed revealed the challenges faced by SIDS. This research addressed the large range of issues, categorised them and provided information and explanation. The findings showed the geography was a crucial element impacting on ICT adoption within higher education of SIDS.

2. The specific factors discovered were documented in Chapter 6. For example, the lack of skilled ICT people and underutilisation of resources were major problem in SIDS due to the small population size. The development of human resources would be central to the deployment of higher education services in remote islands.

3. A TOEG framework based on the original TOE framework was developed due to the literature's lack of theoretical models and frameworks for ICT adoption in 
higher education in SIDS. The framework acted a theoretical background in understanding the context of SIDS.

4. A more detailed model was developed to address the gap in the literature regarding ICT adoption in the higher education sector of SIDS. This model uses the TOEG framework integrated with TAM and DOI. The model was built according to the context of the Maldives, which fits the context of SIDS.

5. A practical roadmap outlining the adoption of ICT in higher education was further developed using the UNESCO continuum model of ICT development with TOEG framework.

\subsection{Limitations of this research}

As with any research, the findings must be measured in light of its limitations.

1. This research was confined to the context of the Maldives as a SIDS. The data was collected from the Maldives and the research participants were Maldivians.

2. The researcher was lecturing at some higher education institutes in the Maldives before starting the doctoral degree course. The researcher acknowledges that this could have led to bias due to familiarity with people among these higher education institutes and also the smallness of the Maldives community.

3. The researcher did not interview expatriate teachers, who comprised a significant part of the teaching community of education sector, or people in the remote islands.

4. Information and Communications Technology is a fairly new concept within the higher education sector in the Maldives. The respondents might not have been aware of trends (e.g. social networking, bring your own device, etc) that might impact in ways that they could not anticipate. 
5. It is possible that respondents might have interpreted questions differently from what was anticipated for the research and might have accidentally provided inaccurate responses.

6. The data was collected qualitatively; hence, the main focus of the study was a small selection of participants from the population for interviews and focus group sessions.

7. There was a risk that the researcher might have introduced bias in translating into English. About half of the interviews and all focus group discussions were carried out in Dhivehi.

8. The recruitment of research participants into this research seemed overly onerous for top management and teachers, as shown by non-availability of people. Time pressures were one factor that might have discouraged top managers from participating in research. Limitations included the availability of potential participants. Some interview participants took some time to consider whether or not they wanted to participate due to lack of understanding about ICT.

9. Finally, the owner of Villa College provided partial funding for this study. Therefore, the researcher feels that it is important to acknowledge the sources of funding, especially as they came from bodies that were also part of the research.

\subsection{Generalisation of findings to other SIDS}

This research can only make modest claims about the generalisability of the research due to a single case research in the Maldives. Klein and Myers (1999, p.75) argued that interpretive research provides a philosophical basis for abstract and generalisation:

“... unique instances can be related to ideas and concepts that apply to multiple situations. Nevertheless it is important that theoretical abstractions 
and generalizations should be carefully related to the field study details as they were experienced and/or collected by the researcher".

This research involved selecting participants from the higher education sector, information technology sector, and private and public sectors, specifically in the Maldives. The researcher undertook 32 interviews and 4 focus group sessions and qualitative survey responses from 100 participants.

Most SIDS face common challenges such as geography, environment and technology. The findings of this research can be generalised to similar SIDS such as Mauritius in the Indian Ocean region and the Marshal Islands in the Pacific region. For example, Mauritius has good Internet penetration rate at $24.8 \%$ with $99 \%$ mobile cellular coverage (see table 10 ).

With these ICT resources Mauritius has the potential to also introduce ICT-enabled higher education. Therefore, the researcher proposed a claim of generalisability for the overall findings of this research to other similar SIDS.

\subsection{Risks of this research}

Although this research has achieved its aim and answered the two research questions, there were some risks. These risks are highlighted here.

Firstly, sound research is an ethical endeavour and the researcher ensured that the interests of research participants were not harmed as a result of the research being done. However, risks might have been encountered due to the small number of people working within the higher education sector.

Secondly, the numbers of people who were available to participate in this research in the Maldives were limited. Therefore, prior and careful planning was necessary and the researcher ensured data collection process ran smoothly with a strict schedule. 
Finally, the cultural and political issues meant some people might have resisted giving an interview due to culture constraints and politics.

\subsection{Contributions of this study}

This study has made some significant contributions to our understanding about the research context. This research has a number of implications for both future research and practice. This study was an attempt to make a small contribution to our understanding and widen knowledge of the need for the adoption of ICT in the higher education sector of SIDS

This research provides evidence grounded in information system theories and frameworks. The findings of this study offer higher education decision makers a framework as they plan and deploy ICT within higher education. This study has brought to light two important research questions, which have never been explored before in the context of the Maldives or SIDS. The research sets out to make the following contributions to knowledge.

\subsubsection{Created a body of knowledge}

There is an extreme lack of academic research undertaken on utilising ICT within the higher education sector of SIDS such as the Maldives. This research has particularly contributed to the existing literature in the context of SIDS, higher education and ICT. This study should be of interest to major stakeholders, policymakers, teachers, students, parents and academic managers at higher education institutes.

\subsubsection{Awareness for utilisation of existing ICT in the Maldives}

Within the higher education sector, teachers, students and academic management already make use of existing ICTs. However, current ICT usage is limited with no strong emphasis on the higher education purpose or on addressing the lack of ICT skills and resistance to change.

This research seeks to bring change to the existing utilisation of ICT on both urban and rural islands within the higher education sector of the Maldives. Through 
qualitative research the participants expressed concerns about ICT resource underutilisation within the higher education sector.

\subsubsection{Factors in the use of ICT in higher education}

A major contribution to the existing knowledge and literature is the identification of factors associated with using ICT in higher education teaching and learning. The factors were e-readiness, ICT resource underutilisation, perception of benefits from the presence ICT infrastructure, ICT policies, digital divide, ICT confidence among teachers, finance, centralisation, human resources, top management support, research, social issues, cultural issues, pedagogy practices, geography and transportation.

\subsubsection{Contribution to the higher education sector}

This research is expected to contribute to the higher education sector of the Maldives in the following ways: (a) identification of specific factors that apply to the use of ICT in higher education; (b) identification of ways in which ICT can be utilised in the Maldives higher education sector; and (c) development of proposed frameworks to address the issues.

\subsubsection{How can ICT be used in higher education sector}

The study has contributed significantly to finding out how ICT could be used in the higher education teaching and learning within the Maldives. This outcome should be useful from an academic and scholarly perspective and for other research studies in the Maldives and also in other SIDS. This researcher realised the importance and this was a major contribution of this study.

\subsubsection{Novelty of developed frameworks and models}

The adoption of a proposed higher education framework certainly is of a great significance to academia. Globally, there is a lack of frameworks or models in the ICT higher education system and there is no comprehensive model that is relevant to developing countries (Czerniewicz \& Brown, 2005).

A major element in the TOEG framework is the introduction of geography, which plays an important role in the SIDS context. Introducing geography into the 
original TOE framework offers a foundation to enable researchers to understand that geography should always be considered in the context of SIDS. The researcher is confident that the TOEG framework will add value to body of knowledge.

The research provides clear descriptions and understanding of existing frameworks of technology and higher education, which have been synthesised from theoretical viewpoints. The study also emphasises how to utilise the existing ICT resources to develop the TOEG framework in the specific context of the Maldives.

It has been highlighted that the current literature lacks generic and applicable models and frameworks necessary for ICT adoption in higher education in the context of SIDS. The primary contribution of this research is the development of a TOEG framework. The framework links issues related to the contextual factor such as technology, organisation, environment and geography.

This TOEG framework has been further enhanced to formulate a model integrating the TOEG framework integrated with TAM and DOI to tackle the four contextual factors, which is a unique contribution by this research. The model shows the complexity of ICT adoption into higher education in the context of SIDS and could help policy makers to better understand and manage their initiatives.

\subsubsection{Attention of researchers}

This research could bring attention to a wider community of researchers in the fields of education and information technology. Due to lack of research, there is a need to undertake studies that could develop new theoretical understanding of the phenomenon in this research context.

\subsection{Implications of the research for SIDS in general}

The contributions to research made by this research primarily focused on the discovered factors, development of a TOEG framework for the adoption of ICT in higher education of SIDS, and practical implications. 
The TOEG framework of adoption of ICT in higher education in this study contributes to provide a blueprint. It offers the potential to future researchers working in this context by offering a framework to be critiqued. In addition, this study has formulated an extended TAM of ICT use in higher education into a SIDS model formulated using the TOEG framework, TAM and DOI is also used in this study.

Finally, the UNESCO continuum model of ICT development was combined with TOEG framework to produce a practical roadmap that could be used in higher education context.

\subsubsection{Research Implications for Ministries of Education}

The Ministries of Education in SIDS should play a key role in creating awareness in order to create a holistic environment for students and teachers. Ministries of Education in SIDS should understand the importance of integrating ICT into higher education systems.

\subsubsection{Research Implications for Higher Education Institutes}

In today's modern world, where the higher education institutes are constantly moving to new technology, they need to face the challenges of inevitable change. The implications are that tertiary institutes should build a shared vision in SIDS, and proper planning would be required for ICT-based teaching and learning. Top managers at higher education institutes should understand the significance of ICT use, to benefit the students, teachers and academic management. The higher education institutes should establish research programmes to evaluate the success of ICT initiatives.

\subsection{Implications of the research for the Maldives}

This research has implications for stakeholders within the higher education sector of ICT integration at different levels: higher education institutes, teachers, students and parents. Details of the recommendations of this study are discussed below.

Organisational culture affects ICT diffusion because top managers perceive the role of ICT as a threat. A change in culture is required to encourage students to use ICT 
facilities in academic learning. ICT friendly top management would be required as well as commitment from the Ministerial level to implement ICT widely in SIDS. It would be necessary to appoint specialised and professional staff in order to spread ICT.

It would also be necessary to develop ICT infrastructures in both public and private tertiary institutes at SIDS. More attention is required in terms of ICT literacy and using ICT higher education at institutes, which cannot be achieved without trained or qualified people in urban and rural areas.

Human resources are required to all level of higher education in SIDS. Integrating ICT in higher education would require more training for teachers, academic staff and senior management at institutes. The process of developing an ICT culture needs to be systematic and sustainable to encourage the ICT acceptance and develop ICT diffusion.

Regulatory bodies such as NCIT, Ministry of Education and EDC should act promptly to formulate sound ICT policies at national and institutional levels. In addition, NCIT should also work across agencies at all levels of government to ensure the development of ICT policies and a regulatory framework that would support the uptake of ICT within the higher education system.

Although the younger generation is more comfortable with mobile technologies, the older teacher generation seems more reluctant. Older generations should also be introduced to mobile technologies and encouraged to utilise and experiment.

The traditional libraries are limited by storage space and also limited to Male'. The students should have access to digital libraries, which would allow the storing of much more information without any physical boundary.

As the islands are scattered and remote in SIDS such as the Maldives, more distance learning or ICT-based higher education teaching and learning tools should be introduced in order to make the classroom more attractive to prospective 
students. The institutes should do more work to overcome the resistance coming from teachers. The modules at institutes could be taught using electronic delivery and establishing an adequate infrastructure, as well as using online networking where students could work interactively and be encouraged to utilise ICT in collaborations.

\subsubsection{Implications for teachers}

This research suggests that teachers should involve themselves in ICT integration within higher education. The adoption of ICT into higher education requires pedagogical changes and teachers becoming technology-friendly. The teachers should be encouraged to widen their ICT knowledge and skills in order to cope with classroom management.

The research shows that, with the given ICT resources, the lack of ICT knowledge among teachers and academic management constitutes a barrier to the use of ICT in higher education. Even where ICT tools were available there was often reluctance to use them appropriately. The need to create ICT awareness and training is necessary for the sustainability of any ICT-enabled technology with the Maldivian culture.

\subsubsection{Implications for academic managers}

The research reaffirmed that support from academic managers is an important factor in ICT integration. The academic managers should play an important role in ICT integration into higher education. Technology integration has implications for academic managers in the development new policy.

It is recommended that academic managers should increase their ICT knowledge and skills and understand the benefits ICT can bring to higher education with modern thinking about how ICT is used in teaching and learning. The academic managers should also ensure a conducive environment for students to encourage innovative explorations. Motivation work by the management would be required to encourage teachers to use technology in their teaching process. 


\subsubsection{Implications for higher education institutes}

The higher education institutes could play a vital role in delivering ICT-based education to students. Given the importance of ICT integration within higher education, it is recommended that higher education institutes should recognise and acknowledge the teachers' central role in ICT integration. The higher education institutes should provide ways to support teachers in developing their ICT skills and knowledge and increasing capability in performing their role.

\subsubsection{Implications for policy makers}

This research provided an understanding of specific factors that affect the integration of ICT into the higher education system. In addition, it has provided a mechanism for how ICT could be used in higher education in the specific context. New practices would have implications for policy makers in the development of new policy and deployment of resources to islands.

It is recommended that policy makers give emphasis to the many students who need higher education on the islands. With the basic ICT infrastructures, the policy makers need to ensure that ICT services are sustainable within higher education. ICT policy should focus on utilising existing ICT infrastructures in the higher education sector and elsewhere. The education policy should focus on spreading higher education through ICT to remote atolls and islands.

\subsubsection{Implications for students}

The research confirmed that students in higher education who live in Male' have a considerable amount of ICT knowledge. Given the urgency for ICT integration within higher education, it is recommended that higher education institutes should take steps in utilising ICT in academic learning rather than spending time on the Internet and social websites. The students should be encouraged to utilise existing ICTs for academic learning.

\subsection{Suggestions for further research}

The qualitative nature of this study explored participants' attitudes, perceptions and beliefs towards the use of ICT in higher education with respect to teaching and 
learning. The researcher would like to outline the following five suggestions for further research:

1. Further research could be undertaken with the same philosophy but focusing on primary and secondary education in the SIDS context. This research primarily focused on the higher education perspective. Carrying out the same research in the primary and secondary education sectors might have different outcomes.

2. An ethnographic study of ICT use in higher education sector in the Maldives through the major ICT initiatives would be an important area to consider.

3. A comprehensive quantitative study of participants' attitudes and behaviour towards using ICT in higher education at island and atoll cluster levels would be an important area to explore. Viewpoints and perceptions from islanders would be important to gain an overall understanding, since the majority of the population of the Maldives live on the remote islands.

4. Due to the unavailability of research it is unclear how ICT resources are utilised within higher education sector. This research has shown that ICT resources are available within the Maldives. However, a detailed study about why ICT resources are underutilised within the higher education sector would also be important in the context of the Maldives.

5. As this research employed a single case (i.e. the Maldives), the same research could be repeated by taking other nations of similar nature (i.e. other SIDS). The culture, social, geography and technology aspect of countries might make a difference in the outcome of the research. For example: Bahrain and Singapore are listed as SIDS but might have different geography, culture and social aspects. A good example of another SIDS close to the Maldives is Mauritius where the geographical nature is similar. 
6. Massive Open Online Courses (MOOC) is a new concept for delivering learning content through the Internet to anyone who wants to take a course anywhere. MOOC provides a platform for making online course materials available freely such as videos to enable interactive user forums for students, teachers and academic management. MOOC were not well known at the time of data gathering during this research.

MOOCs will be one important tool, which could be used in the delivery of higher education within SIDS such as the Maldives. MOOC are relevant to SIDS, because they can deliver courses through non-synchronous access, and also other learning materials, exams and assessments through online forums.

The remote learners in the islands of the Maldives require access to higher education through online platforms. Research that focuses on utilising MOOC in the context of SIDS would be an important study, especially in the context of the Maldives.

\subsection{Final Comments}

ICT is used in the higher education sector in many developed and developing countries. The developed countries are equipped with state-of-the-art technologies while developing countries have varying degree of resources. A review of the literature suggested that there is lack of studies undertaken in the context of SIDS and developing countries context. In this research, the researcher looked at the context of the Maldives only as an example of SIDS.

The findings presented in Chapters 6 could be important to other SIDS countries with the same scenario. The researcher urges other researchers to continue similar studies. There is a scarcity of research on ICT adoption within higher education of SIDS and developing countries. It is important that SIDS integrate ICT into their higher education to truly realise the substantial benefits.

ICT can play an important role in finding solutions for our problems in tertiary education and for students stationed on the islands. Due to diseconomies of scale 
in the remote islands and the unlikely chance of developing tertiary institutes on each island, SIDS like the Maldives are forced to move to a technology solution.

In general, this research has contributed by: a) identifying the factors associated with adopting ICT into higher education; b) suggesting how ICT can be utilised in the context of higher education; c) providing a TOEG framework integrated with the TAM and DOI for ICT adoption within higher education in SIDS; d) utilising UNESCO's continuum model of ICT development and TOEG framework to ground the factors and themes to produce a practical roadmap; and e) providing recommendations for policy makers, top management within the higher education sector, teachers, students and higher education institutes.

Due to the geographical nature of the islands in the Maldives, the only solution left is to utilise ICT in higher education. The researcher believes that establishing "virtual island higher education institutes" across the nation using ICT would overcome the existing problems, enabling higher education to be made available and affordable to everyone.

The adoption of ICT in higher education would create innumerable opportunities for the students, teachers and the higher education sector. This could also address the social problems faced such as overcrowding and unemployment issues.

\subsection{Chapter Summary}

This concluding chapter began with a brief overview of the researcher's motivation for this particular research. This section was followed by reaffirming the answers to the main research questions in this study followed by the possible limitations and risks of this research. The chapter ended with the possibility of generalising the findings to other SIDS contexts, research contributions, research implications, suggestions for further research and final comments by the researcher.

The researcher provided clear justifications to support the arguments of this study by connecting the research problems, research objectives, assertions, model, research design, data sources and analyses, findings, research claims, implications 
and recommendations. To summarise, the researcher hopes this study has provided a useful contribution to the existing body of knowledge. 


\section{REFERENCES}

Adam, M. S., Urquhart, C. (2007). IT capacity building in developing countries: A model of the Maldivian tourism sector. Information Technology for Development, 13(4), 315-335.

Aduwa-Ogiegbaen, S. E., \& Iyamu, E. O. S. (2005). Using information and communication technology in secondary schools in Nigeria: Problems and prospects. Educational Technology \& Society, 8(1), 104-112.

Ageel, I. (2006). Drug rehabilitation and practice dilemmas in the Maldives. (Unpublished Master's of Social Science in Psychology thesis, University of Waikato, Hamilton, New Zealand).

Ainley, J., Enger, L., \& Searle, D. (2008). Students in a digital age: Implication of ICT for teaching and learning. In J. Voogt \& G. Knezek (Eds.), International handbook of information technology in primary and secondary education (pp. 63-80). New York, NY: Springer.

Albirini, A. (2006). Cultural perceptions: The missing element in the implementation of ICT in developing countries. International Journal of Education and Developing using Information and Communication Technology (IJEDICT), 2(1), 49-65.

Alebaikan, R., \& Troudi, S. (2010). Blended learning in Saudi universities: Challenges and perspectives. ALT-J, Research in Learning Technology, 18(1), 4959.

Aneh Dhivehi Rajje. (2009-2013). The Strategic action plan. National framework for Development 2009-2013. The President's Office, Male': The Maldives. Retrieved from http://www.presidencymaldives.gov.mv/Documents/APEN.pdf

Arpacı, İ., \& Yardımcı, Y., Özkan, S., \& Türetken, Ö. (2012). Organizational adoption of mobile communication technologies, Paper presented at European, Mediterranean \& Middle Eastern Conference on Information Systems 2012 (EMCIS2012), 7-8 June, Munich, Germany.

Asian Development Bank - ADB. (2010). Key indicators for Asia and the Pacific. Manila, Philippines: ADB.

Atchoarena, D., Graca, P. D. D., \& Marquez, J. M. (2008). Strategies for post-primary education in small island developing states (SIDS): Lessons from Cape Verde, Comparative Education, 44(2), 167-185.

Athif, A., \& Pimenidis, E. (2009). E-Crime threats on e-Government - the case of the Maldives. In R. Perryman, H. Mouratidis, S. W. Lee, C Dragova, D. Palmer-Brown, D.C Wijeysekera, H Saidpout, S. J Dodds \& W Hosny (Eds.), Proceedings of 
Advances in Computing Technology, The School of Computing, Information Technology and Engineering, $4^{\text {th }}$ Annual Conference, (pp. 16-25). London, UK: University of East London.

Awa, H. 0., \& Ukoha, O. (2012). Integrating TAM and TOE frameworks and expanding their characteristic constructs for e-Commerce adoption by SME's. Proceedings of Informing Science \& IT Education Conference (InSITE) 2012, (pp. 571-588). Montreal, Canada: John Molson School of Business, Concordia University.

Bacchus, M. K. (2008). The education challenges facing small nation states in the increasingly competitive global economy of the twenty-first century. Comparative Education, 44(2), 127-145

Balasubramanian, K., Clarke-Okah, W., Daniel, J., Ferreira, F., Kanwar, A., Kwan, A., Lesperance, J., Mallet, J., Umar, A., \& West, P. (2009). ICTs for higher education, Vancouver, BC: Commonwealth of Learning.

Bates, T. (2000a). Learning and teaching and the impact of technology. EDUCASE Review, 38-43.

Bates, T. (2000b). Taking control: Managing teaching technologies: Strategies for college and university leadership. San Francisco, CA: Jossey-Bass Publishers.

Bates, T. (2001a). International distance education: Cultural and ethical issues. Distance Education, 22(1), 122-136.

Bates, T. (2001b). The continuing evolution of ICT capacity: The implications for education. In G. M. Farrell (Ed.) The changing face of virtual education Vancouver, B.C: Commonwealth of Learning.

Bates, T. (2001c). National strategies for e-learning in post-secondary education and training. Fundamentals of Educational Planning 70. Paris, France: Institute of Education Planning, United Nations, Educational, Scientific and Cultural Organization (UNESCO).

Bates, M., Manuel, S., \& Oppenheim, C. (2007). Models of early adoption of ICT innovations in higher education. Ariadne, 50, 1-12.

Benbasat, I., \& Barki, H. (2007). Quo vadis, TAM. Journal of the Association for Information Systems, 8(4), 211-218.

Benbasat, I., Goldstein, D. K., \& Mead, M., (1987). The case research strategy in studies of information systems. MIS Quarterly, 11(3), 369-386.

Benson, V., Anderson, D., \& Ooms, A. (2011). Educators' perceptions, attitudes and practices: Blended learning in business and management education, Research in Learning Technology, 19(2), 143-154. 
Blurton, C. (1999). New directions of ICT-use in education. UNESCO. Retrieved from http://www.unesco.org/education/educprog/lwf/dl/edict.pdf.

Binger, A., Wit, M., Briguglio, L., Bhuglah, A., Paeniu, B., Gibbons, L., Ali, M., \& Howard, R. (2002). The growing vulnerability of small island developing states. The United Nations Development Programme (UNDP) - Capacity 21 Project, Kingston, Jamaica: University of West Indies Centre for Environment and Development.

Bingimlas, K. (2009). Barriers to the successful integration of ICT in teaching and learning environments: A review of the literature. Eurasia Journal of Mathematics, Science \& Technology Education, 5(3), 235-245.

Bonoma, T. V. (1985). Case research in marketing opportunities. Journal of Marketing Research, 22(2), 199-208.

Breen, R., Lindsay, R., Jenkins, A., \& Smith, P. (2001). The role of information and communication technologies in a university learning environment. Studies in Higher Education, 26(1), 95-114.

Burgess, J. R. D., \& Russell, J. E. A. (2003). The effectiveness of distance learning initiatives in organisations. Journal of Vocational Behavior, 63, 289-303.

CAM. (2013). The communication authority of the Maldives. Retrieved from http://www.cam.gov.mv/Statistics.htm

Cambridge Education. (2005). TA 4338 - MLD: Strengthening the framework of education towards Vision 2020: Final report. Manila, The Philippines: Asian Development Bank (ADB).

Chan, P. S., \& Welebir, B. (2003). Strategies for e-Education. Industrial and Commercial Training, 35(5), 196-202.

Caudill, J. G. (2007). The Growth of m-Learning and the growth of mobile computing. International Review of Research in Open and Distance Learning, $8(2), 1-13$.

Cavaye, A. L. M. (1996). Case study research: A multi-faceted research approach for IS. Information Systems Journal, 6(3), 227-242.

Central Intelligence Agency - CIA. (2012). The World Fact Books. Retrieved from https://www.cia.gov/library/publications/the-world-factbook/geos/cq.html

Chang, C. C., \& Yan, C. F., \& Tseng, J. S. (2012). Perceived convenience in an extended Technology Acceptance Model: Mobile technology and English learning for college students. Australasian Journal of Educational Technology, 28(5), 809-826. 
Chau, P.Y.K., \& Tam, K.Y. (1997). Factors affecting the adoption of open systems: An exploratory study, MIS Quarterly, 21(1), 1-24.

Chen, S. C., Li, S. H., \& Li, C. Y. (2010). Recent related research in Technology Acceptance Model, Australian Journal of Business and Management Research, 1(9), 124-127.

Cho, J., \& Trent, A. (2006). Validity in qualitative research revisited. Qualitative Research, 6(3), 319-340.

Clique College. (2013). Clique College. Retrieved from http://www.cliquecollege.edu.mv/downloads/

Clough, G., Jones, A. C., McAndrew, P., \& Scanlon, E. (2008). Informal learning with PDAs and smartphones. Journal of Computer Assisted Learning, 24(5), 359-371.

Cochrane, T. D. (2010). Exploring mobile learning success factors. ALT-J, Research in Learning Technology, 18(2), 133-148.

Coffin, R. J., \& MacIntyre, P. D. (1999). Motivational influences on computer-related affective states. Computers in Human Behavior, 15(5), 549-569.

Country Report. (2009, February). Maldives: Country report. Facing global and local challenges: The new dynamics for higher education. Paper presented at UNESCO South, South-West and Central Asia Sub-regional Preparatory Conference for 2009 World Conference on Higher Education, New Delhi, India.

Creswell, J. W. (2003). Research design: Qualitative, quantitative and mixed methods approaches. ( $2^{\text {nd }}$ ed.). Thousand Oaks, CA: Stage Publications Inc.

Creswell, J. W. (2009). Research design: Qualitative, quantitative and mixed methods approaches. ( $3^{\text {rd }}$ ed.). Thousand Oaks, CA: Sage Publications South Asia Edition.

Creswell, J. W., \& Clark, K. L. P. (2007). Designing and conducting mixed methods Research. (2 ${ }^{\text {nd }}$ ed.). Thousand Oaks, CA: Stage Publications Inc.

Crotty, M. (1998). The foundations of social research: Meaning and perspective in the research process. Thousand Oaks, CA: Stage Publications Inc.

Cullen, R., (2001). Addressing the digital divide. Online Information Review, 25(5), 311-320.

Czerniewicz, L., \& Brown, C. (2005). Access to ICT for learning and teaching: From single artefact to inter-related resources. International Journal of Education and Development using Information and Communication Technology (IJEDICT), 1(2), 42-56.

Czerniewicz, L., Ravjee, N., \& Mlitwa, N. (2007). Information and communication technologies (ICTS) and South African higher education: Understandings (of) the 
landscape. Pretoria, South Africa: Council of Higher Education. Retrieved from http://www.che.ac.za/documents/d000146/Review_HE_SA_2007_Complete.pd f

Dada, D. (2006). E-readiness for developing countries: Moving the focus from the environment to the users. The Electronic Journal of Information Systems in Developing Countries, 27 (6), 1-14.

Dale, C. (2008). iPods and creativity in learning and teaching: An instructional perspective. International Journal of Teaching and Learning in Higher Education, 20(1), 1-9.

Damanpour, F. (1992). Organizational size and innovation, Organization Studies, $13,375-402$.

Darke, P., Shanks, G., \& Broadbent, M. (1998). Successfully completing case study research: combining rigour, relevance and pragmatism. Information Systems Journal, 8(4), 273-289.

Davis, F. D. (1989). Perceived usefulness, perceived ease of use, and user acceptance of information technology. MIS Quarterly, 13(3), 319-340.

Davis, F. D. (1993). User Acceptance of Information Technology: system characteristics, user perception and behavioural impacts, International J. Man Machine Studies, 38, 475-487.

Davis, F. D., Bagozzi, R. P., \& Warshaw, P. R. (1989). User acceptance of computer technology: A comparison of two theoretical models. Management Science, 35(8), 982-1003.

Decentralisation Act. (2010). The Maldives Decentralisation Act. Maldives Gazette, 39(52), 1-58.

Dede, C. (2008). Theoretical perspective in influencing the use of information technology in teaching and learning. In J. Voogt \& G. Knezek (Eds.), International handbook of information technology in primary and secondary education, (pp. 43-62). New York, NY: Springer.

Demiraslan, Y., \& Usleul, Y. K. (2008). ICT integration processes in Turkish schools: Using Activity Theory to study issues and contradictions. Australasian Journal of Educational Technology, 24(4), 458-474.

Denscombe, M. (1999). The good research guide: For small-scale social research projects. Maidenhead, UK: Open University Press.

Denzin, N. K., \& Lincoln, Y. S. (2000). Introduction: The discipline and practice of qualitative research. In N. K. Denzin \& Y. S. Lincoln (Eds.), Handbook of qualitative research (2nd ed., pp. 1-28). Thousand Oaks, CA: Sage Publications Inc. 
Department of National Planning. (2010a). Key economic indicators 2010. Male', Department of National Planning, Maldives.

Department of National Planning. (2010b). Statistical year book 2010. Male': The Maldives: Department of National Development. Retrieved from http://planning.gov.mv/yearbook2010/yearbook/

Department of National Planning. (2011). Seventh national development plan 2006 2010: Creating new opportunities. (NDP), Maldives. Retrieved from http://www.planning.gov.mv/en/images/stories/ndp/seventh_ndp.pdf

Department of National Planning. (2012a). Statistical year book 2012. Male': The Maldives: Department of National Development. Retrieved from http://planning.gov.mv/yearbook2012/yearbook.html

Department of National Planning. (2012b). Key economic indicators 2012. Male', Department of National Planning, Maldives. Retrieved from http://planning.gov.mv/publications/keyeconomicindicators/2012/October\% 202012.pdf

Department of National Planning. (2012c). Mapping Maldives. Male', Department of National Planning, Maldives. Retrieved from http://planning.gov.mv/atlas/raster/downloads/Map\%20of\%20Maldives \%202009\%207.2\%2008\%20K.jpg

Doh, S., \& Stough, R. R. (2010). Analysis of the impact of the perceived usefulness of ICT in the digital divide between disabled and non-disabled people in South Korea. International Review of Public Administration, 4(3), 53-70.

Dooley, L. M. (2002). Case study research and theory building. Advances in Developing Human Resources, 14(3), 335-354.

Douglas, R. (2011). ICT for teaching and ICT for learning: They are not the same. Computers in New Zealand Schools, 23(2), 126-136.

Dubé, L., \& Paré, G. (2003). Rigor in information systems positivist case research: Current practices, trends, and recommendations. MIS Quarterly, 27(4), 597635.

Duch, H. (2005). Consultation in international development: The case of early childhood in Maldives. School Psychology International, 26(2), 178-191.

Dudding, C. C. (2009). Digital videoconferencing: Applications across the disciplines. Communication Disorders Quarterly, 30(3), 178-182.

Eisenhardt., K. M. (1989). Building theories from case study research. The Academy of Management Review, 14(4), 532-550. 
Elnaggar, A. (2008). Towards gender equal access to ICT. Information Technology for Development, 14(4), 280-293.

Erstad, O. (2003). Electracy as empowerment: Student activities in learning environments using technology. Young, 11(1), 11-28.

Fagoonee, I. (2001). E-Learning in a small island state: The case of Mauritius, Proceedings of the International Conference on Engineering Education, Oslo, Norway, Session 8B4,(pp. 5-9). Retrieved from: http://www.ineer.org/events/icee2001/proceedings/papers/171.pdf

Farrell, G., \& Wachholz, C. (2003). Meta-survey on the use of technologies in education in Asia and the Pacific. Bangkok, Thailand: Asia Pacific Regional Bureau for Education, UNESCO.

FASHAN \& NCB. (2003). Rapid situation of drug abuse in Maldives 2003. Male'. The Maldives: UNESCP \& UNDP.

Flecknoe, M. (2002). ICT doing different things, rather than doing them differently, Management in Education. 16(1), 26-30.

Flick, U. (2009). An introduction to qualitative research (4th ed.). London, UK: Sage South Asia Edition.

Fulu, E. (2007). Gender, vulnerability, and the experts: Responding to the Maldives tsunami. Development and Change, 38(5), 843-864.

Gaible, E. (2009a). Survey of ICT and education in the Caribbean: Regional trends and analysis, Information for Development, Vol I, Washington, DC: World Bank.

Gaible, E. (2009b). Survey of ICT and education in the Caribbean: Regional trends and analysis, Vol II, Washington, DC: World Bank.

Godoe, O., \& Johansen, T. S. (2012). Understanding adoption of new technologies: Technology readiness and technology acceptance as an integrated concept. Journal of European Psychology Students, 3, 38-52.

Godschalk, D. R., \& Lacey, L. (2001). Learning at a distance: Technology impacts on planning education. Journal of Planning Education and Research, 20(4), 476489.

Golafshani, N. (2003). Understanding reliability and validity in qualitative research. The Qualitative Report, 8(4), 597-607. Retrieved from http://www.nova.edu/ssss/QR/QR8-4/golafshani.pdf

Goldfarb, A. (2006). The (teaching) role of universities in the diffusion of the Internet. International Journal of Industrial Organization, 24(2), 203-225. 
Gordon, M. (2008). Between constructivism and connectedness, Journal of Teacher Education, 59(4), 322-330.

Graham, C. R (2005). Blended learning systems: Definition, current trends, and future directions. In C. J. Bonk \& C. R Graham (Eds.), Handbook of blended learning: Global perspectives (pp.1-21). San Francisco, CA: Pfeiffer Publishing. Retrieved from http://media.wiley.com/product_data/excerpt/86/07879775/0787977586.pd f

Gregor, S. (2006). The nature of theory in information systems. MIS Quarterly, 30(3), 611-642.

Gulati, S. (2008). Technology-enhanced learning in developing nations: A review. International Review of Research in Open and Distance Learning, 9(1), 1-16.

Gulbahar, Y. (2007). Technology planning: A roadmap to successful technology integration in schools. Computers \& Education, 49(4), 943-956.

Guri-Rosenblit, S. (2006). "Distance education" and "e-learning": Not the same thing. High Education, 49, 467-493.

Guttman, C. (2003). Education in and for the information society. UNESCO Publication for the World Summit on the Information Society. Paris, France: UNESCO.

Hayes, D. N. A. (2007). ICT and learning: Lessons from Australian classrooms. Computers \& Education, 49(2), 385-395.

HaveeruOnline. (2009). Wataniya brings education to the masses with "m-Learning". 5(13), 1-3.

Hefzallah, I. M. (2004). The new educational technologies and learning: Empowering teachers to teach and students to learn in the information age ( $2^{\text {nd }} \mathrm{ed}$.).

Springfield, IL: Charles C Thomas Publisher Ltd.

Henderson, J C. (2008). The politics of tourism: A perspective from the Maldives. Tourismos: An International Multidisciplinary Journal of Tourism, 3(1), 99-115.

Herselman, M., \& Britton, K. G. (2002). Analysing the role of ICT in bridging the digital divide amongst learners. South African Journal of Education, 22(4), 270274.

Hirscheim, R. A. (Ed.). (1992). Information systems epistemology: An historical perspective. Oxford, UK: Blackwell Scientific Publications.

Hofstede, G. H. (1991). Cultures and organizations: Software of the mind. London, UK: McGraw-Hill Book Company. 
Hong, K-S., \& Songan, P. (2011). ICT in the changing landscape of higher education in Southeast Asia. Australasian Journal of Educational Technology, 27(8), 12761290.

HRCM. (2009). Rapid assessment of the employment situation in the Maldives. Human Rights Commission of the Maldives, Male': The Maldives. Retrieved from http://www.hrcm.org.mv/Publications/otherreports/TheEmploymentSituatio nintheMaldivesEng.pdf

Hsu, J. (2007). Innovative technologies for education and learning. International Journal of Information and Communication Technology Education, 3(3), 70-89.

Hsu, P. F., Kraemer, K. L. \& Dunkle, D. (2006). Determinants of e-business use in U.S, firms. International Journal of Electronic Commerce, 10(4), 9-45.

Hu, L., \& Webb, M. (2009). Integrating ICT to higher education in China: From the perspective of Activity Theory. Education Information Technology, 14(2), 143161.

Hussain, A. S. (2008). Governance and vulnerability in small island states. (Unpublished PhD thesis, Victoria University of Wellington, Wellington, New Zealand).

Hussain, D. (2008). Constitution of the Republic of Maldives. Ministry of Legal Reform, Information and Arts. Retrieved from http://www.maldivesinfo.gov.mv/home/upload/downloads/Compilation.pdf

Hussein, A. (2009). The use of triangulation in social sciences research: can qualitative and quantitative method be combined. Journal of Comparative Social Work, 1, 1-12.

Ilomaki, L., \& Rantanen, P. (2007). Intensive use of ICT in school: Development differences in students' ICT expertise. Computers \& Education, 48(1), 119-136.

Ibrahim, M. (2006). Maldives. In Digital Review of Asia Pacific 2005/2006 (pp. 160168). New Delhi, India: Sage.

Ibrahim, M. (2010, June). Workshop on e-Government for achieving the MDG's and ecities network in Asia and Pacific: Local e-Government for the MDGs and service delivery. Paper presented at the International Conference on Good Governance for National Development. Korea.

InfoDev. (2010a). Survey of ICTs for education in India and South Asia: Country studies. The World Bank. Retrieved from:

http://www.infodev.org/en/Publication.880.html 
InfoDev. (2010b). Information and communication technology for education and South Asia. Policy coherence in the application of ICTs for education. Washington, DC: The World Bank.

Internet World Stats. (2013). World Internet usage and population statistics. Retrieved from http://www.internetworldstats.com/stats.htm

Iqbal, S., \& Qureshi, I. A. (2012). M-learning adoption: A perspective from developing country. The international Review of Research in Open and Distance Learning, 13(3), 147-164.

ITU. (2004). Information and communication technology in the atolls: Maldives case study. Geneva, Switzerland: International Telecommunication Union (ITU).

ITU. (2007). ITU annual report. Geneva, Switzerland: International Telecommunication Union (ITU).

ITU. (2008). Use of information communication technology by the world's children and youth: A statistical compilation. Geneva, Switzerland: International Telecommunication Union (ITU).

ITU. (2009). Measuring the information society. The ICT development Index. Geneva: Switzerland: International Telecommunication Union.

ITU. (2010). Measuring the Information Society. Geneva, Switzerland: International Telecommunication Union (ITU).

Jaffer, S., Ng'ambi, D., \& Czerniewicz, L. (2007). The role of ICTs in higher education in South Africa: One strategy for addressing learning and teaching challenges. International Journal of Education and Development using Information and Communication Technology (IJEDICT), 3(4), 131-142.

Johnson, B., \& Christensen, L. (2008). Educational research: Quantitative and qualitative, and mixed approaches ( $3^{\text {rd }}$ ed.). Thousand Oaks, CA: Sage Publication Limited.

Jones, S., \& Cresse, E. L. (2001). E-education: Creating partnership for learning. Melbourne, VIC: School of Information Management, RMIT University, 1-16.

Jung, 0. (2005). ICT-pedagogy integration in teacher training: Application cases worldwide. Educational Technology \& Society, 8(2), 94-101.

Kearney, M., Schuck, S., Burden, K., \& Aubusson, P. (2012). Viewing mobile learning from a pedagogical perspective. Research in Learning Technology, 20, 1-17.

Khan, S. H., Hasan, M., \& Clement, C. K. (2012). Barriers to the introduction of ICT into education in developing countries: the example of Bangladesh. International Journal of Instruction. 5(2), 61-80. 
Kington, A., Harris, S., \& Leask, M. (2002). Innovative practice using ICT in schools: Findings from two case studies. Management in Education. 16(1), 31-35.

Kirkman, G. S. (2002, May). Network readiness and small island developing states. Paper presented at the Small Island Economies in the Era of Globalization Conference. Center for International Development at Harvard University, Cambridge, MA.

Kirkwood, A., \& Price, L. (2006). Adaptation for a changing environment: Developing learning and teaching with information and communication technologies. International Review of Research in Open and Distance Learning, $7(2), 1-14$.

Klein, H. K., \& Myers, M. D., (1999). A set of principles for conducting and evaluating interpretive field studies in information systems. MIS Quarterly, 23(1), 67-94.

Knowlton, N. (2008). Pedagogy, technology and value in higher education. Paper presented at Outcomes of Higher Education: Quality Relevance and Impact Conference. Paris, France. Retrieved from: http://www.oecd.org/dataoecd/3/45/41218281.pdf

Koohang, A., Riley, L., \& Smith, T. (2009). E-learning and constructivism: From theory to application, Interdisciplinary Journal of E-Learning and Learning Objects, 5, 91-109.

Koonjul, J. (2004). The special case of small island developing states for sustainability development. Natural Resources Forum, 28(2), 155-156.

Kozma, R. (2002). ICT and educational reform in developed and developing countries. Retrieved from http://web.udg.edu/tiec/orals/c17.pdf

Kozma, R. (2005). National policies that connect ICT-based education reform to economic and social development. Human Technology, 1(2), 117-156.

Kozma, R., \& Anderson, R. (2002). Qualitative case studies of innovative pedagogical practices using ICT. Journal of Computer Assisted Learning, 18(4), 387-394.

Kozma, R., McGhee, R., Quellmalz, E., \& Zalles, D. (2004). Closing the digital divide: Evaluation of the World Links program. International Journal of Educational Development, 24(4), 361-381.

Kozma, R. \& Wagner, D. (2008). Reaching the most disadvantaged with ICT: What worked? In R. Sweet and D. Wagner (Eds.). ICT in non-formal and adult education: Supporting out-of-school youth and adults. (pp. 1-21), Paris, France: OECD. 
Lai, K. W. (2011). Digital Technology and the culture of teaching and learning in higher education. Australasian Journal of Educational Technology, 27(8), 12631275.

Lane, A. (2009). The impact of openness and bridging educational digital divides. International Review of Research in Open and Distance Learning, 10(5), 1-12.

Lippert, S. K., \& Govindarajulu, C. (2006). Technological, organizational, and environment antecedents to web services adoption, Communications of the IIMA, 6(1), 146-158.

Littlejohn, A. H. (2002). Improving continuing professional development in the use of ICT. Journal of Computer Assisted Learning, 18(2), 166-174.

Liu, C. C (2010). Evolution of constructivism, Contemporary Issues in Education Research, 3(4), 63-66.

Liu, T. C., Wang, H. Y., Liang, J. K., Chan, T. W., Ko, H. W., \& Jang, J. C. (2003). Wireless and mobile technologies to enhance teaching and learning. Journal of Computer Assisted Learning, 19(3), 371-382.

Looker, E. D. (2008). Gender and information technology. In J. Voogt \& G. Knezek (Eds.) International handbook of information technology in primary and secondary education, (pp. 779-788). New York, NY: Springer.

Loxley, W., \& Jullien, P. (2004). Information and communication technologies in education and training in Asia and the Pacific. Manila, The Philippines: Asian Development Bank (ADB).

Mac-Ikemenjima, D. (2005, April). E-education in Nigeria: Challenges and prospects. Paper presented at $8^{\text {th }}$ UN ICT Tasks Force Meeting. Dublin, Ireland.

McKinnon, D. H., Nolan C. J. P., \& Sinclair, K. E. (2000). A longitudinal study of student attitudes towards computers: Resolving an attitude decay paradox. Journal of Research on Computing in Education, 32(3), 325-335.

Mattar, J. A. (2010). Constructivism and connectivism in education technology: Active, situated, authentic, experiential and anchored learning. Retrieved from http://www.joaomattar.com/Constructivism\%20and\%20Connectivism\%20in \%20Education\%20Technology.pdf

MCHE. (2008). Maldives college of higher education (MCHE). Maldives. Retrieved from http://www.mche.edu.mc

MCST. Ministry of Communication, Science and Technology (MCST). Maldives. (2005a). Education: science and technology master plan. Retrieved from http://www.mcst.gov.mv/Downloads/Documents/S\&T/Part203Information_Technology.pdf 
MCST. Ministry of Communication, Science and Technology (MCST). Maldives. (2005b). Information technology: Science and technology master plan. Retrieved from http://www.mcst.gov.mv/Downloads/Documents/S\&T/Part206Education.pdf

Melhuish, K. \& Falloon, G. (2010). Looking to the future: M-learning with the iPad. Computers in New Zealand Schools, 22(3), 1-16.

Meyer, C. B. (2001). A case in case study methodology. Field Methods, 13(4), 329352.

Merriam, S. B. (1988). Case study research in education: A qualitative approach. San Francisco, CA: Jossey-Bass Publishers.

Microsoft. (1997). Colleges and universities face challenges to prepare students for technology-driven world, redefine instruction using technology and develop digital infrastructure. Retrieved from http://www4.gu.edu.au:8080/adtroot/uploads/approved/adt-QGU20060804.162650/public/05Main.pdf.

Minges, M., \& Gray, V. (2004). Information and communication technology in the atolls: Maldives case study (pp. 1-58). Geneva, Switzerland: International Telecommunication Union (ITU). Retrieved from http://www.itu.int/ITUD/ict/cs/maldives/material/CS_MDV.pdf

Mlitwa, N. B. W. (2007). Technology for learning and teaching in higher education contexts: Activity Theory and Actor Network Theory analytical perspectives. International Journal of Education and Development using Information and Communication Technology (IJEDICT), 3(4), 54-70.

MNU. (2011). The Maldives national university. Retrieved from http://mnu.edu.mv/images/the_uni/annualreports/2011.pdf

MOE. (2012). School Statistics 2011. Ministry of education. Retrieved from http://moe.gov.mv/root/assets/JQuploader/server/php/files/2929.pdf

Mohamed, A. M. (2005, May). Country report of Maldives. Paper presented at Eight Session of the Regional Committee for the Regional Convention of Recognition of Qualifications, Kunming, China.

Mohamed, N. (2006). An exploratory study of the interplay between teachers' beliefs, instructional practices \& professional development. (Unpublished $\mathrm{PhD}$ thesis, University of Auckland, Auckland, New Zealand).

Moore, J. (2005). Is higher education ready for transformative learning? Journal of Transformative Education, 3(1), 76-91.

Morgan, D. L., \& Spanish, M. T. (1984). Focus groups: A new tool for qualitative research. Qualitative Sociology, 7(3), 253-270. 
MPND. (2006). Ministry of Planning \& National Development (MPND), Maldives. (2006). Statistics year book of Maldives 2006. Retrieved from http://www.planning.gov.mv/publications/yrb2006/yrb05/all.htm

MPND. (2007). Ministry of Planning \& National Development (MPND), Maldives. (2007). Seventh national development plan (2006 - 2010). Retrieved from http://www.planning.gov.mv/en/images/stories/ndp/seventh_ndp.pdf

MQA. (2009). Maldives national qualification framework (MNQF). Maldives Qualifications Authority. Retrieved from: http://www.mqa.gov.mv/downloads/MNQF2009withAmmendment1.pdf

Ministry of Transport and Communication (MTC). (2006). Maldives telecommunication policy 2006 - 2010. Retrieved from http://www.tam.gov.mv/downloads/Telecom_Policy_2006_Eng.pdf

MTCA. (2008). Ministry of tourism and civil aviation (MTCA). Maldives. Retrieved from http://www.tourism.gov.mv/article.php?aId=33

Myers, M. D. (2006). Qualitative research in information systems. Retrieved from http://www.qual.auckland.ac.nz

Myers, M. D., \& Tan, F. B. (2002). Beyond models of national culture in information systems research. Journal of Global Information Management, 10(1), 24-32.

Mustonen-Ollila, E., \& Lyytinen, K. (2003). Why organizations adopt information system process innovations: A longitudinal study using Diffusion of Innovation theory, Information Systems Journal. 13(3), 275-297

Naismith, L., Lonsdale, P., Vavoula, G., \& Sharples, M. (2004). Literature review on mobile technologies and learning. Bristol, UK: NESTA Futurelab.

Nandhakumar, J., \& Jones, M. (1997). Too close for comfort? Distance and engagement in interpretive information systems research. Information Systems Journal, 7(2), 109-131.

Nanjappa, A., \& Grant, M. M. (2003). Constructing on constructivism. Electronic Journal for the Integration of Technology in Education, 2(1), 38-56.

NCIT. (2013). e-Government. The National Centre for Information Technology. Male', The Maldives. Retrieved from http://202.21.186.162/index.php/en/egovernment

Narcotics Control Bureau. (2006). Maldives drug control master plan 2006 -2010. Male', The Maldives: National Narcotics Control Bureau, with technical assistance from United Nations Office on Drugs and Crime. Retrieved from http://www.health.gov.mv/PDF/HIV/Drug\%20Master\%20Plan\%202006\%20$\% 202010 \% 20$ Maldives.pdf 
Neuman, W. L. (1997). Social research methods. Qualitative and quantitative Approaches. ( $3^{\text {rd }}$ ed.). Boston, MA: Allyn and Bacon.

Neuman, W. L. (2011). Social research methods. Qualitative and quantitative Approaches. (7 $7^{\text {th }}$ ed.). Pearson Education Inc.

Oh, C. H. (2003). Information communication technology and the new university: A view on eLearning. The ANNALS of the American Academy of Political and Social Science, 585(1), 134-153.

Olakulehin, F. K. (2007). Information communication technologies in teacher training and professional development in Nigeria. Turkish Online Journal of Distance Education - TOJDE, 8(1), 133-142.

Oliveira, T. \& Martins, M, F. (2011). Literature review of information technology adoption models at firm level. The Electronic Journal Information Systems Evaluation, 14(1), 110- 121.

Oliver, R. (2002). The role of ICT in higher education for the 21st century: ICT as a change agent for education. Proceedings of the Higher Education for the 21st Century Conference. (pp. 1-8). Miri, Sarawak: Curtin University.

Olutimayin, J. (2002). Adopting modern information technology in the South Pacific: A process of development, preservation, or underdevelopment of the culture? The Electronic Journal of Information Systems in Developing Countries, $9(3), 1-12$.

OHCHR. (2007). Office of the United Nations High Commissioner for Human Rights. Retrieved from http://www2.ohchr.org/english/law/pdf/cescr.pdf

Panday, P. P. (2009). Simplifying podcasting. International Journal of Teaching and Learning in Higher Education, 20(2), 251-261.

Paré, G. (2004). Investigating information systems with positivist case study research. Communications of the Association for Information Systems, 13(1), 233-264.

Park, Y. (2011). A pedagogical framework for mobile learning: Categorizing educational applications of mobile technologies into four types. International Review of Research in Open and Distance Learning, 12(2), 78-102.

Pearce, K., \& Scutter, S. (2010). Podcasting of health science lectures: Benefits for students from non-English speaking background. Australasian Journal of Educational Technology, 26(7), 1028-1041.

Pee, L. G., \& Kankanhalli, A. (2010). Bridging the digital divide: Use of public internet kiosks in Mauritius. Journal of Global Information Technology, 18(1), 15-38. 
Pickard, A. J. (2007). Research methods in information. London, UK: Facet Publishing.

Pillay, H., \& Elliot, B. (2005). Looking through old lenses to understand the emerging new world order: Implications for education reform in small island state. Journal of Research in International Education, 4(1), 87-106.

Plumm, K. M. (2008). Technology in the classroom: Burning the bridges to the gaps in gender-biased education? Computers \& Education, 50(3), 1052-1068.

Podmore, V. N. (1991). 4-years olds, 6-years-old, and microcomputers: A study of perceptions and social behaviours. Journal of Applied Development Psychology, 12(1), 87-101.

Prain, V., \& Hand, B. (2003). Using new technologies for learning: A case study of a whole-school approach. Journal of Research in Technology in Education, 35(4), 441-458.

Pudjianto, B. and Hangjung, Z. (2009, December). "Factors affecting e-government assimilation in developing countries. Paper presented at the $4^{\text {th }}$ Communication Policy Research, South Conference, Negombo, Sri Lanka.

Punch, K. F. (2005). Introduction to social research: Quantitative and qualitative approaches (2nd ed.). Thousand Oaks, CA: Sage Publication Ltd.

Punie, Y. (2007). Learning Spaces: An ICT-enabled model of future learning in the knowledge-based society. European Journal of Education, 42(2), 185-199.

Punie, Y., Zinnbauer., \& Cabrera, M. (2006). A review of the impact of ICT on learning. Working paper for DG EAC. Retrieved from http://ftp.jrc.es/EURdoc/JRC47246.TN.pdf

Raouf, J. B., Naser, I. S., \& Jassim, B. K. (2012). Determinants of e-Learning implementation success in the Iraqi MOHE. Eng. \& Tech Journal, 30(4), 659671.

Reddi, U. V., \& Sinha, V. (2005). Maldives ICT use in Education: UNESCO metasurvey on the use of technologies in education, 253-256.

Rogers, E. M. (1995). Diffusion of innovations (4th ed.). New York, NY: The Free Press.

Rogers, E. M. (2001). The digital divide. Convergence: The International Journal of Research into New Media Technologies, 7(4), 96-111.

Rogers, E. M. (2003). Diffusion of innovations ( $5^{\text {th }}$ ed.). New York, NY: The Free Press. 
Rogers, E., \& Scott, K. (1997). The diffusion of innovations model and outreach from the national network of libraries of medicine to Native American communities. Retrieved from http://nnlm.gov/archive/pnr/eval/rogers.html

Roman, R. (2003). Diffusion of innovations as a theoretical framework for telecenters. Information Technologies and International Development, 1(2), 5366.

Rowland, B. (2003). Employing interpretive research to build theory of information systems practice. Australasian Journal of Information Systems, 10(2), 3-22.

Rumble, G. (2001). Just how relevant is e-education to global educational needs. Open Learning, 16(3), 223-232.

Sahin, I. (2006). Detailed review of Rogers' diffusion of innovations theory and educational technology-related studies based on Rogers' theory. The Turkish Online Journal of Educational Technology - TOJET, 5(2), 14-23.

Salomon, G. (1991). Transcending the qualitative - quantitative debate: The analytic and systemic approaches to educational research. Educational Researcher, 20(6), 10-18.

Sangrà, A., \& González-Sanmamed, M. (2010). The role of information and communication technologies in improving teaching and learning processes in primary and secondary schools, ALT-J, Research in Learning Technology, 18(3), 207-220.

Santos-Paulino, A. U. (2011). Fragility and vulnerability in small island developing states: Issues and challenge. Paper presented at First Meeting of the Caribbean Development Round Table, ECLAC, 13 September 2011, Port of Spain.

Saunders, G., \& Klemming, F. (2003). Integrating technology into a traditional learning environment: Reasons for and risks of success. Active Learning in Higher Education, 4(1), 74-86.

Schofield, J. W. (1997). Psychology: Computers and classrooms social processes - A review of the literature. Social Science Computer Review, 15(1), 27-39.

Scutter, S., Stupans, I., Sawyer, T., \& King, S. (2010). How do students use podcasts to support learning? Australasian Journal of Educational Technology, 26(2), 180191.

Seale, C. (1999). Quality in qualitative research. Qualitative Inquiry, 5(4), 465-465478.

Sealy, W. C. (2003). Empowering development through e-government: Creating smart communities in small island states. International Forum and Library Review, 35(2-4), 335-358. 
Seidman, I. (1998). Interviewing as qualitative research (2nd ed.). New York, NY: Teachers College Press.

Selwyn, N. (2007). The use of computer technology in university learning and teaching: a critical perspective. Journal of Computer Assisted Learning, 23(2), 83-94.

Shareef, M., Ojo, A., \& Janowski, T. (2010). Exploring the digital divide in Maldives. Proceedings of the 9th IFIP Human Choice and Computers International Conference (HCC9 2010), World Computer Congress 2010 (WCC2010), 20-23 September 2010. (pp. 1-14), Brisbane, QLD: IFIP Series, Springer.

Shareef, A. F. (2005). Distance education on the remote islands of Maldives. (Unpublished PhD thesis, Massey University, Palmerston North, New Zealand).

Shareef, A. F., \& Kinshuk. (2002, December). Distance education system for remote islands in Maldives. Paper presented at International Conference on Computers in Education (ICCE), Massey University, Palmerston North, New Zealand. Retrieved from http://www.col.org/pcf3/Papers/PDFs/Shareef_Kinshuk.pdf

Shareef, A. F., \& Kinshuk. (2006). Distance education in the Maldives: Learner support for students in island communities. In H. H Lund, E. Sutinen, M. Duveskog, Kinshk \& A. N. E Mkocha (Eds.), Proceedings of the $4^{\text {th }}$ IEEE International Workshop on Technology for Educating in Developing Countries (TEDC'06) (pp.24-25). Los Alamitos, CA: IEEE Computer Society.

Shinohara, F., \& Nan-Zhao, Z. (2006, June-July). Research and development of the Asia-Pacific regional guidelines of ICT-pedagogy integration and teacher training. Paper presented at $18^{\text {th }}$ Annual Japan-US Teacher Education Consortium, Tokyo, Japan.

Sidorenko, A., \& Findlay, C. (2001). The digital divide in East Asia. Asian-Pacific Economic Literature, 15(2), 18-30.

Sife, A. S., Lwoga, E. T., \& Sanga, C. (2007). New technologies for learning and teaching: Challenges for higher learning institutions in developing countries. International Journal of Education and Development using Information and Communication Technology (IJEDICT), 3(2), 57-67.

Slay, H., Sieborger, I., \& Hodgkinson-Williams, C. (2008). Interactive whiteboards: Real beauty or just "lipstick"? Computers \& Education, 51(3), 1321-1341.

Solvberg, A. M. (2003). Computer-related control beliefs and motivation: A panel study. Journal of Research on Computing in Education, 35(4), 473-487.

Somekh, B. (2008). Factors affecting teachers' pedagogical adoption of ICT. In J. Voogt \& G. Knezek (Eds.), International handbook of information technology in primary and secondary education (pp. 449-460). New York, NY: Springer. 
South, J. H., Ho. C. P., \& Narita, S. (2008). A distance learning enrichment: A Pacific Perspective. In J. Voogt \& G. Knezek (Eds.) International handbook of information technology in primary and secondary education (pp. 713-724). New York, NY: Springer.

Stake, R. E. (1995). The art of case study research. Thousand Oaks, CA: Sage Publications Ltd.

Stensaker, B., Maasen, P., Borgan, M., Oftebro, M., \& Karseth, B. (2007). Use, updating and integrating of ICT in higher education: Linking purpose, people and pedagogy. Higher Education, 54(3), 417-433.

Stuart, I., McCutcheon, D., Hanfield, R., McLachlin, R., \& Samson, D. (2002). Effective case research in operations management: a process perspective. Journal of Operations Management, 20(5), 419-433.

Sumner, J. (2000). Serving the system: A critical history of distance education. Open Learning, 15(3), 267-285.

Sutherland, R., Armstrong, V., Barnes, S., Brawn, R., Breeze, N., Gall, M., Mathewman, S., Olivero, F., Taylor, A., Triggs, P., Wishart, J., \& John, P. (2004). Transforming teaching and learning: Embedding ICT into everyday classroom practices. Journal of Computer Assisted Learning, 20(6), 413-425.

Swan, K. (2005). A constructivist model for thinking about learning online. In J. Bourne \& J. C. Moore (Eds), Elements of Quality Online Education: Engaging Communities. Needham, MA: Sloan-C.

TAM. (2008). Telecommunication statistics: Telecommunications authority of Maldives. Retrieved from http://www.tam.gov.mv/articles.php?artID=39

TAM. (2011). Telecommunication statistics. Telecommunications authority of Maldives. Retrieved from http://www.tam.gov.mv/articles.php?artID=39

Tam, M. (2000). Constructivism, instructional design, and technology: Implications for transforming distance learning. Educational Technology \& Society, 3(2), 50 60. Retrieved from http://www.ifets.info/journals/3_2/tam.html

Taylor, J. C. (1994). Distance education technologies: The fourth generation. Higher Education Management, 6(2), 179-190.

Taylor, J. C. (2001). Fifth generation distance education. Higher Education Series Report No40. Toowoomba: QLD: Global Learning Services, University of Southern Queensland.

Teichler, U. (2006). Changing structures of the higher education systems: The increasing complexity of underlying forces. Higher Education Policy, 19, 447461. 
Tinio, V. L. (2000). ICT in education. Asia-Pacific development information programme: UNDP. Retrieved from

http://www.apdip.net/publications/iespprimers/ICTinEducation.pdf

Tornatzky, L. G. \& Fleischer, M. (1990). The processes of technological innovation, Idaho Falls, ID: Lexington Books.

Trochim, W. M. K. (2006). Research methods knowledge Base: Qualitative validity: Retrieved from http://www.socialresearchmethods.net/kb/qualval.php

UNCTAD. (2004a). E-commerce and development report 2004. United Nations Conference on Trade and Development: New York and Geneva, 95-132

UNCTAD. (2004b). Is a special treatment of small island developing States possible? United Nations Conference on Trade and Development: New York and Geneva, 1-102.

UNCTAD. (2009). Information economy report: Trends and outlook in turbulent times. United Nations Conference on Trade and Development: New York and Geneva, 1-153.

UNDP. (2000). Maldives human development report 2000: Challenges and responses. Male', The Maldives: Ministry of Planning and National Development (MPND) \& United Nations Development Programme (UNDP).

UNESCAP. (2008). Statistical year book for Asia and the Pacific. United Nations Economic and Social Commission for Asia and the Pacific: Bangkok, Thailand: UNESCAP.

UNESCO. (2000). Globalisation and living together: The challenges for educational content in Asia. United Nations Education, Scientific, and Cultural Organization. Delhi: India: UNESCO. Retrieved from http://unesdoc.unesco.org/images/0014/001472/147277e.pdf

UNESCO. (2002). Information and communication technology in education: A curriculum for schools and programme of teacher development. United Nations Education, Scientific, and Cultural Organization. Paris: France: UNESCO. Retrieved from http://unesdoc.unesco.org/images/0012/001295/129538e.pdf

UNESCO. (2005). United Nations Education, Scientific, and Cultural Organization UNESCO (2005). Towards knowledge societies: UNESCO world report. Paris, France: UNESCO.

UNESCO. (2010, November). World data on education: $7^{\text {th }}$ edition. UNESCO world report. United Nations Education, Scientific, and Cultural Organization. Paris, France: UNESCO. 
UNESCO. (2011). Tertiary Education in Small States, IIEP Policy Brief. United Nations Education, Scientific, and Cultural Organization, Paris, France: UNESCO.

UNICEF. (2010). UNICEF Maldives country office. UN building, Maldives. Retrieved from http://www.unicef.org/infobycountry/maldives_41944.html

UN-OHRLLS. (2011). Small Island Developing States - Office of the High Representative for the Least Developed Countries, Landlocked Developing Countries and Small Island Developing States (UN-OHRLLS). New York: UNOHRLLS.

Uys, P. M., Nleya, P., \& Molelu, G. B. (2004). Technological innovation and management strategies for higher education in Africa: Harmonizing reality and idealism. Education Media International, 41(1), 67-80.

Valentine, G., \& Holloway, S. (1999). "The vision things": Schools and information and communication technology. Convergence: The International Journal of Research into New Media Technologies, 5(4), 63-79.

Van de Ven, A. H. (1986). Central problems in the management of innovation, Management Science, 32(5), 590-607.

Venkatesh, V. (2000). Determinants of perceived ease of use: Integrating control, computer anxiety and enjoyment into the Technology Acceptance Model, Information Systems Research, 11(4), 342-365.

Venkatesh, V., \& Bala, H. (2008). Technology Acceptance Model 3 and a research agenda on interventions, Decision Sciences, 39(2), 273-315.

Venkatesh, V., Morris, M., Davis, G. B., \& Davis F. D. (2003). User acceptance of information technology: Toward a unified view. MIS Quarterly, 27(3), 425-478.

Villa College. (2013). Villa College. Retrieved from http://villacollege.edu.mv/index.php/about-villa-college

Visagie, S., \& Villers, de C. (2010, October). The consideration of Facebook as an academic tool by ICT lecturers across five countries. Paper presented at SACLA Conference, University of Pretoria, South Africa.

Von Tigerstrom, B. (2005). Small island developing sates and international trade: Special challenges in global partnership for development. Melbourne Journal of International Law, 6(2), 402-436.

Voss, C., Tsikriktsis, N., \& Frohlich, M. (2002). Case research in operations management. International Journal of Operations \& Production Management, 22(2), 195-219.

Waheed, M. (2009, July). Tertiary education in small states: Planning in the context of globalization: Keynote address. IIEP Policy Forum, Paris, France. 
Walsham, G. (1995a). Interpretive case studies in IS research: Nature and method. European Journal of Information Systems, 4, 74-81.

Walsham, G. (1995b). The emergence of interpretivism in IS research. Information Systems Research, 6(4), 376-394.

Walsham, G. (2006). Doing interpretive research. European Journal of Information Systems, 15, 320-330.

Walsham, G., Roby, D., \& Sahay, S. (2007). Special issue on information systems in developing countries. MIS Quarterly, 31(2), 317-326.

Walsham, G., \& Sahay, S. (2006). Research on information systems in developing countries. Information Technology for Development, 12(1), 7-24.

Wang, Q. (2008). A generic model for guiding the integration of ICT in learning and teaching. Innovations in Education and Teaching International, 45(4), 411-419

Wataniya. (2011). Broadband category 3G+. Retrieved from http://www.wataniya.mv/category/3G

Wataniya. (2012). M-learning. Retrieved from http://www.wataniya.mv/page/products/mlearning

Watson. J. (2008). Promising practices in online learning: Blended learning: The convergence of online and face-to-face education, North American Council for Online Learning. Retrieved from http://www.inacol.org/research/promisingpractices/NACOL_PPBlendedLearning-lr.pdf

Webb, M., \& Cox, M. (2004). A review of pedagogy related to information and communications technology. Technology, Pedagogy and Education, 13(3), 235286.

Weber, R. (2004). The rhetoric of positivism versus interpretivism: A personal view. MIS Quarterly, 28(1), 1-10.

Willis, S., \& Tranter, B. (2006). Beyond the "digital divide": Internet diffusion and inequality in Australia. Journal of Sociology, 42(1), 43-59.

Wilson, V. (1997). Focus groups: A useful qualitative method for educational research. British Educational Research Journal, 23(2), 209-224.

Wims, P., \& Lawler, M. (2007). Investing in ICTs in educational institutions in developing countries: An evaluation of their impact in Kenya. International Journal of Education and Development using Information and Communication Technology (IJEDICT), 3(1), 5-22.

Wong, P. P. (2011). Small island developing states. Opinion, (2), 1-6 
World Bank. (2000). Small states: Meeting challenges in the global economy. Report of the Commonwealth Secretariat / World Bank joint task force on small states. Washington: USA. The World Bank

World Bank. (2011). Human capital for a knowledge society higher education in the Maldives: An evolving seascape. Washington, DC: The World Bank and Human Development Unit, South Asian Region.

World Health Organization (WHO). (2006). WHO-AIMS report on mental health system in Maldives, Male', The Maldives: World Health Organization \& Ministry of Health Maldives.

Yin, R. K. (1994). Case study research: Design and method (2nd ed.). Thousand Oaks, CA: Sage Publication, Inc.

Yin, R. K. (2003). Case study research: Design and methods (3 $3^{\text {rd }}$ ed.). Thousand Oaks, CA: Sage Publication, Inc. 


\section{APPENDICES}

\section{Appendix 1: Interview Questions and Guidelines}

\section{Interview Questions}

\begin{tabular}{|c|c|}
\hline AREA & INTERVIEW QUESTIONS \\
\hline Technology & $\begin{array}{l}\text { To your knowledge what is the existing ICT usage within } \\
\text { the higher education sector in learning and teaching of the } \\
\text { Maldives? }\end{array}$ \\
\hline Organisation & $\begin{array}{l}\text { What are challenges (problems) currently faced in dealing } \\
\text { and using ICT in the Maldives higher education sector? }\end{array}$ \\
\hline $\begin{array}{l}\text { Technology, } \\
\text { Organisation, } \\
\text { Environment }\end{array}$ & $\begin{array}{l}\text { Any additional comment or information to help me } \\
\text { understand how the existing ICT is utilised in higher } \\
\text { education learning and teaching in the unique context of } \\
\text { the Maldives? }\end{array}$ \\
\hline Environment & $\begin{array}{l}\text { What are the perceived barriers currently faced in dealing } \\
\text { and using ICT in the higher education learning and } \\
\text { teaching in the unique geographical context of the } \\
\text { Maldives? Please explain }\end{array}$ \\
\hline Environment & $\begin{array}{l}\text { What are the perceived drivers in the use of ICT in higher } \\
\text { education learning and teaching? Please explain }\end{array}$ \\
\hline Technology & $\begin{array}{l}\text { Given the resources why do you think ICT is underutilised } \\
\text { in the Maldives among students, teachers and others? } \\
\text { Please describe. }\end{array}$ \\
\hline $\begin{array}{l}\text { Technology and } \\
\text { Organisation }\end{array}$ & $\begin{array}{l}\text { How can ICT be best used as a tool to assist learning and } \\
\text { teaching in the Maldives higher education sector? Please } \\
\text { explain }\end{array}$ \\
\hline $\begin{array}{l}\text { Technology and } \\
\text { Organisation }\end{array}$ & $\begin{array}{l}\text { What is your perception of ICT use and use of technology } \\
\text { in the Maldives higher education sector? Please explain }\end{array}$ \\
\hline Technology & $\begin{array}{l}\text { Do you think the use of ICT would improve higher } \\
\text { education learning and teaching in the Maldives? How? }\end{array}$ \\
\hline Technology & In your experience what is the best way to utilise ICT as \\
\hline
\end{tabular}




\begin{tabular}{|c|c|}
\hline & tool in higher education learning and teaching? \\
\hline $\begin{array}{l}\text { Technology and } \\
\text { Environment }\end{array}$ & $\begin{array}{l}\text { Do you have any negative feelings or thoughts about the } \\
\text { use of ICT in higher education learning and teaching in the } \\
\text { Maldives? Please describe. }\end{array}$ \\
\hline $\begin{array}{l}\text { Technology, } \\
\text { Organisation, } \\
\text { Environment }\end{array}$ & $\begin{array}{l}\text { Is there anything else you would like to say about how ICT } \\
\text { can be used in the Maldives higher education sector? }\end{array}$ \\
\hline $\begin{array}{l}\text { Technology and } \\
\text { Organisation }\end{array}$ & $\begin{array}{l}\text { What model or a strategy best integrates existing and } \\
\text { future ICTs into the Maldives higher education sector? }\end{array}$ \\
\hline $\begin{array}{l}\text { Technology and } \\
\text { Organisation }\end{array}$ & $\begin{array}{l}\text { What are the perceived benefits a practical / sustainable } \\
\text { model would bring to the higher education sector in the } \\
\text { Maldives? }\end{array}$ \\
\hline $\begin{array}{l}\text { Technology, } \\
\text { Organisation } \\
\text { Environment }\end{array}$ & $\begin{array}{l}\text { What are the perceived problems in formulating a practical } \\
\text { / sustainable higher education sector model in the } \\
\text { Maldives? }\end{array}$ \\
\hline $\begin{array}{l}\text { Technology, } \\
\text { Organisation and } \\
\text { Environment }\end{array}$ & $\begin{array}{l}\text { Finally, does existing ICT use help in achieving the goals of } \\
\text { the Maldives higher education in learning and teaching? } \\
\text { How? }\end{array}$ \\
\hline
\end{tabular}

\section{Interview Guidelines}

The following guidelines will be in place for interviews.

\section{Introductory Components:}

- Choose a venue with least distraction

- Explain the purpose of the interview to the participant. Some participants might want to know the probable interview questions.

- The confidentiality should be explained to the participant and the researcher should guarantee confidentiality.

- Explain the probable time it will take to the participant

- Provide contact information of the researcher

- Prepare the audio-recoding digital recorders

- Obtain signature of consent 


\section{Question Format}

- No more than 15 open-ended questions are listed

- Most of the time ask for facts than opinion

- Always use probes as needed

\section{Closing Components}

- Inform the participant that interview transcript will be provided for verification

- Thank all the participants

\section{Appendix 2: $\quad$ Focus Group Questions and Guidelines}

\section{Focus Group Questions}

\begin{tabular}{|l|l|}
\hline \multicolumn{1}{|c|}{ AREA } & \multicolumn{1}{|c|}{ FOCUS GROUP QUESTIONS } \\
\hline Technology & $\begin{array}{l}\text { To your knowledge what is the existing ICT usage within } \\
\text { the higher education sector in learning and teaching of the } \\
\text { Maldives? }\end{array}$ \\
\hline Organisation & $\begin{array}{l}\text { What are challenges (problems) currently faced in dealing } \\
\text { and using ICT in the unique context of Maldives higher } \\
\text { education sector? }\end{array}$ \\
\hline Environment & $\begin{array}{l}\text { What are the perceived barriers currently faced in dealing } \\
\text { and using ICT in the higher education learning and } \\
\text { teaching in the unique geographical context of the } \\
\text { Maldives? Please explain }\end{array}$ \\
\hline Environment & $\begin{array}{l}\text { What are the perceived drivers in the use of ICT in higher } \\
\text { education learning and teaching? Please explain }\end{array}$ \\
\hline $\begin{array}{l}\text { Technology } \\
\text { Organisation }\end{array}$ & $\begin{array}{l}\text { Given the resources why do you think ICT is underutilised } \\
\text { in the Maldives among students, teachers and others? } \\
\text { Please describe. }\end{array}$ \\
\hline and \\
teaching in the Maldives higher education sector? Please \\
explain
\end{tabular}




\begin{tabular}{|l|l|}
\hline Technology & $\begin{array}{l}\text { Do you think the use of ICT would improve higher } \\
\text { education learning and teaching in the Maldives? How? }\end{array}$ \\
\hline Technology & $\begin{array}{l}\text { What model or a strategy best integrate existing and future } \\
\text { ICT's into the Maldives higher education sector? }\end{array}$ \\
\hline $\begin{array}{l}\text { Technology and } \\
\text { Organisation }\end{array}$ & $\begin{array}{l}\text { What are the perceived benefits a practical / sustainable } \\
\text { model would bring to the higher education sector in the } \\
\text { Maldives? }\end{array}$ \\
\hline $\begin{array}{l}\text { Technology, } \\
\text { Organisation } \\
\text { Environment }\end{array}$ & $\begin{array}{l}\text { What are the perceived problems in formulating a practical } \\
\text { / sustainable higher education sector model in the } \\
\text { Maldives? }\end{array}$ \\
\hline
\end{tabular}

\section{Focus Group Guidelines}

The following guidelines will be in place to ensure smooth focus group discussions.

\section{Introductory Components:}

- Explaining the purpose of the focus group discussion to the participant.

- Moderator kick-off the session.

- The confidentiality should be explained to the participants and the researcher should guarantee the confidentiality.

- Explain the probable time it will take for the focus group.

- Prepared the audio-recoding digital recorders.

- Obtain signature of consent

- No observers are allowed

- No one besides the researcher and the moderator have access to the participants' comments

\section{Question Format:}

- No more than 10 open-ended questions are listed

- Most of the time ask for facts than opinion

- Always use probes as needed 


\section{Closing component:}

- Moderator and the researcher thank all the participants 

treated confidential. Access to the data will be restricted to the investigator and his two supervisors

\section{SAMPLE SURVEY FORM}

\section{SECTION A - DEMOGRAPHIC INFORMATION (Please tick the appropriate boxes)}

1. Name (Optional):

Contact No (Optional):

2. Type: Stakeholder:

$\square$

e-Mail (Optional):

Teacher: $\square$

3. Gender: Male: $\square$ Female: $\square$

4. Age:

$$
\begin{aligned}
& 16-25 \square \\
& 46-55 \square
\end{aligned}
$$

$26-35$

$56-65$

$36-45$

66 over

5. Name of Institution / Faculty:

6. Course \& Level:

7. What is the duration you have been using computers and related ICTs in learning and teaching in higher education sector?

Less than 06 months

02 years

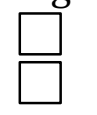

06 months

03 years
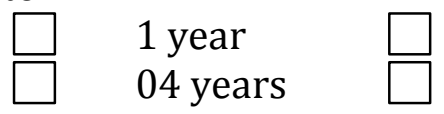

8. How often do you use Internet?

Never $\square$ Once a week

Other (specify):

\section{Several times a week $\square \quad$ Daily}

9. What are the existing ICT peripherals (devices) used?

Multimedia Projector

Interactive Whiteboards $\square$

Video conferencing

Mobile devices

Other (specify):
Computers

Digital cameras

Printers

Video cameras

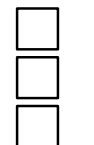

DVD

TV

Scanners

10. For what purpose do you use existing ICTs?

Finding learning Resources

Interacting with students and teachers

Communication with others

Research

Professional Development

Administration

Other (specify): 


\section{SECTION B - ICT USAGE}

11. Please describe your existing ICT usage within the higher education sector?

12. What are challenges (problems) currently faced in dealing and using ICT in the Maldives higher education sector?

13. Given the resources why do you think ICT is underutilised in the Maldives among students, teachers and others? Please describe.

14. How can ICT be best used as a tool to assist learning and teaching given the unique context in higher education of the Maldives? Please explain

15. What practical model or a strategy best integrate existing and future ICTs into the Maldives higher education sector? Please describe 


\title{
Appendix 4: Information sheet for Qualitative Survey with Consent
}

\author{
Victoria \\ UNIVERSITY OF WELLINGTON \\ Te Whare Wānanga \\ o te Ūpoko o te Ika a Māui

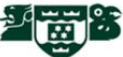 \\ Victoria University of Wellington \\ School of Information Management \\ Information Sheet - Qualitative Survey
}

[Date]

Dear [participant]

Project Title: The use of Information and Communication Technology in learning and teaching within Higher Education sector of a Small Island Developing State:

\section{The Case of the Maldives}

With the permission of your institution, I invite you to participate in this research. Let me first introduce myself and give you a brief overview of this research. I am a PhD student at Victoria University of Wellington in New Zealand. I am conducting research entitled "The use of Information and Communication Technology in learning and teaching within Higher Education sector of a Small Island Developing State: The Case of the Maldives, and I seek your participation as a participant in Qualitative Survey.

The main objectives of this research are: a) to explore the existing situation of ICT usage in higher education sector of Maldives; b) to discover how ICT can be better utilised in learning and teaching of Maldives higher education; and c) to devise a suitable model for future use in the higher education sector of the Maldives.

Confidentiality of participating organisations and of participating individuals will be preserved as a matter of course. However, given the smallness of the higher education community in the Maldives, it may always be possible for members of the community to guess at the individuals and their organisations by the data they 
provide. This risk needs to be understood by participants but it will be minimised wherever possible.

Participants are members of the higher education sector, IT sector and relevant agencies in the Maldives. I hope to get knowledge of the perceptions and expectations how ICT can be utilised in the higher education learning and teaching. The institution in which you work / study has given permission for you to participate in my research if you are willing.

The purpose of this qualitative survey is to find out the existing ICT utilisation with the Maldives higher education sector. In other words the qualitative survey is designed to identify and assess how ICT is currently used within the Maldives higher education. Participation is voluntary; any participants may withdraw without having to give reasons at any stage of the survey.

The Human Ethics Committee of the School of Information Management at Victoria University of Wellington has granted ethical approval subject for this research.

The qualitative survey forms will be distributed with this information sheet. If you choose to participate, submission of the completed form will be taken to indicate consent. Please complete the survey form and return it to me personally or drop to the box provided within the institute.

If you have any questions or would like to receive further information about the project, please contact Mohamed Kinaanath on +9607775353 or via email at mohamed.kinaanath@vuw.ac.nz or my supervisors:

1. Associate Professor, Dr. Hans Lehmann, at the School of Information Management at Victoria University of Wellington, by phone: + 6444635879 or via email hans.lehmann@vuw.ac.nz or via post. 
2. Senior Lecturer, Dr. Brian Harmer, at the School of Information Management at Victoria University of Wellington, by phone: + 6444635887 or via email brian.harmer@vuw.ac.nz or via post.

My Contact Details: Mr. Mohamed Kinaanath PhD Candidate

M. Gaahaka, Shaheed Ali Hingun

Male', Maldives

Phone: (960) 7775353

e-mail: mohamed.kinaanath@vuw.ac.nz mkinaanath@gmail.com

Best Regards

Mohamed Kinaanath 


\section{Appendix 5: Human Ethics Approval}

TE WHARE WĀNANGA O TE ŪPOKO O TE IKA A MĀUI

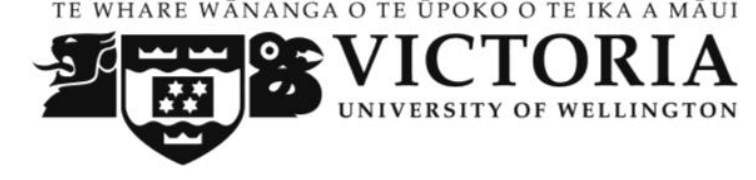

SIM HUMAN ETHICS COMMITTEE

\section{Comments on Application for Human Ethics Approval}

Date: 1 May 2009

RE: The use of Information and Communication Technology in learning and teaching within Higher Education of a Small Island Developing State: The case of the Maldives

Principal Researcher: Mohamed Kinaanath

Supervisor (student research): Hans Lehmann; Brian Harmer

Ref No: \#16398

Approval Given after Amendments made - Application accepted.

Human Ethics Approval valid until: (Date: as in application or no more than 3 years)

Thank you for the amendments you have made to your HEC application. These meet the committee's required changes. On behalf of the HEC Chair I am authorised to inform you that you may now proceed with your research. You may begin your data collection immediately but please note that a hard copy of your application signed by both you and your supervisor (or other researchers involved for staff applications) is required within one month before approval can be recorded.

Wendy Chen

HEC Administrator

SIM Human Ethics Committee 
Appendix 6: Request for participation for Higher Education Institutes

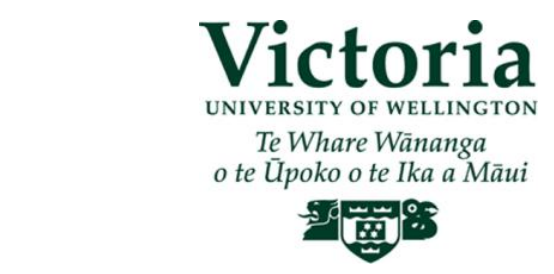

Victoria University of Wellington

School of Information Management

Request for Higher Education Institutions to Participate in Doctoral Research

Dear $<$ participant $>$

$<$ Date $>$

\section{Subject: Approval to undertake research at your organisation}

I am seeking approval to undertake research in your organisation. I am a $\mathrm{PhD}$ student in the School of Information Management at Victoria University of Wellington, New Zealand. As part of this research, I would like to invite your institution to participate. The Project Title is: "The use of Information and Communication Technology in Learning and Teaching within Higher Education Sector of a Small Island Developing State: The Case of the Maldives".

The proposed study venue is in the Republic of the Maldives. The research will be carried out using selected participants from the higher education institutes, and other key stakeholders in the Maldives. I will be selecting participants from a selection of the country's higher education institutes.

Confidentiality of participating organisations and of participating individuals will be preserved as a matter of course. However, given the smallness of the higher education community in the Maldives, it may always be possible for members of the community to guess at the individuals and their organisations by the data they provide. This risk needs to be understood by participants but it will be minimised wherever possible.

The main objectives of this research are: a) to explore the existing situation of ICT usage in higher education sector of Maldives; b) to discover how ICT can be utilised 
in learning and teaching of Maldives higher education; and c) to devise a suitable model for future use in the higher education sector of the Maldives.

The Human Ethics Committee of the School of Information Management at Victoria University of Wellington requires me to obtain your informed consent. I am therefore seeking your consent for members of your institution to be allowed to participate in this research. If that permission is granted, I will invite appropriate individuals to participate in qualitative surveys, interviews and focus groups. The participants will be able to check transcripts of interviews and may withdraw anytime before 1 July 2009.

If individual participants choose to withdraw from individual participation any information provided up to that time will be destroyed and omitted from my research. Focus group participants, however, will be unable to withdraw information provided once the group has commenced working.

The participants in the qualitative survey for the students will be chosen from your institute. To approach the students I will visit your institute to meet with the students and will distribute the survey forms in their respective classes. I will give them a brief of my doctoral research and the objective of this qualitative survey. Therefore, please grant permission to conduct the survey in the classroom session.

The survey forms and consent form will be distributed in the class and participants will be asked to return the completed forms at some future time. No student will be forced to participate in this survey and they complete the survey form at their own free will.

The survey forms and consent form will distributed in the class and ask them to return the completed forms at some future time if they wish freely to participate. No student will be forced to participate in this survey and are complete the survey forms at own will. 
To obtain the appropriate background knowledge, I also seek your permission to review relevant documents from your organisations as you see fit to provide them. Confidentiality of the information provided is assured.

If you agree to your organisation's participation, it would be helpful if you would nominate a suitably qualified person to be my prime contact.

Information derived from the collected, collated and analysed data may be published as case studies, as articles in academic journals, and presented at conferences. The thesis will be submitted for marking to the School of Information Management and deposited in the university library. In addition, a copy of the thesis will be lodged in Victoria University of Wellington electronic repository.

Any information or opinion provided by participants will not be attributed to them and neither they nor their institution will be identified in any way without appropriate permission. A copy of the written notes and the transcription of each interview you participate in will be sent to participants for review and feedback. They will have the opportunity to correct any written notes or transcripts of recorded sessions to ensure accuracy. Through the project, raw data will be kept under password protection and destroyed two years after the completion of the project.

I will be grateful if you could send me your notice of acceptance before $18^{\text {th }}$ May 2009. If you have any questions or would like to receive further information about the project, please contact me on +9607775353 or via email at mohamed.kinaanath@vuw.ac.nz, or my supervisors:

1. Associate Professor, Dr. Hans Lehmann, at the School of Information Management at Victoria University of Wellington, by phone: + 6444635879 or via email hans.lehmann@vuw.ac.nz or via post. 
2. Senior Lecturer, Dr. Brian Harmer, at the School of Information Management at Victoria University of Wellington, by phone: + 6444635887 or via email brian.harmer@vuw.ac.nz or via post.

Yours sincerely

Mohamed Kinaanath 


\title{
Appendix 7: Consent of Higher Education Institutes
}

\author{
Victoria \\ UNIVERSITY OF WELLINGTON \\ Te Whare Wänanga \\ o te Ūpoko o te Ika a Māui \\ 59: \\ Victoria University of Wellington \\ School of Information Management \\ Consent to participate in Research - Institutions
}

Project Title: THE USE OF INFORMATION AND COMMUNICATION TECHNOLOGY IN LEARNING AND TEACHING WITHIN HIGHER EDUCATION SECTOR OF A SMALL ISLAND DEVELOPING STATE: THE CASE OF THE MALDIVES

On behalf of the institution, I have been given and have understood an explanation of this research project. I have had an opportunity to ask questions and have had them answered to my satisfaction. I understand that we may withdraw the institution and any information its members have provided without having to give reasons as long as I do it by 1 July 2009. I understand that if we withdraw from the project, any data we have provided will be destroyed by Mohamed Kinaanath and not used for this research or any other purpose.

I understand that any information provided by members of this institution will be kept confidential to individual or group concerned, the researcher and to his supervisors. I understand that the information provided will be used for this research project only and that any further use will require further written consent. I understand that the recording of interviews and focus group discussions will be electronically wiped two years after the conclusion of the project.

Confidentiality of participating organisations and of participating individuals will be preserved as a matter of course. However, given the smallness of the higher education community in the Maldives, it may always be possible for members of the community to guess at the individuals and their organisations by the data they provide. This risk needs to be understood by participants but it will be minimised wherever possible. 
[Please mark with a $(\checkmark)$ to indicate agreement]

- I agree to permit members of groups of members to participate in this research

- I understand that the participants will have an opportunity to check the transcripts of the interviews

- I would like to receive a summary of the results of this research when it is completed

- I understand that the data we provide will not be used for any other purpose or released to others without our written consent

- I understand a copy of the thesis will be lodged in Victoria University of Wellington electronic repository

Chose where appropriate/applicable:

- I understand the published results may use the name of our organisation, and opinions can be attributed to our organisation.

OR

- I understand the published results will not use the name of our organisation, and no opinions will be attributed to our organisation in any way that will identify us, without our written consent.

Signature:

Name of the participant:

Date: 


\title{
Appendix 8: Information sheet for interviews
}

\author{
Victoria \\ UNIVERSITY OF WELLINGTON \\ Te Whare Wānanga \\ o te Ūpoko o te Ika a Māui \\ 50箅圈: \\ Victoria University of Wellington \\ School of Information Management \\ Information Sheet - Interviews
}

[Date]

Dear [participant]

Project Title: THE USE OF INFORMATION AND COMMUNICATION TECHNOLOGY IN LEARNING AND TEACHING WITHIN HIGHER EDUCATION SECTOR OF A SMALL ISLAND DEVELOPING STATE: THE CASE OF THE MALDIVES

With the permission of your institution, I invite you to participate in this research. Let me first introduce myself and give you a brief overview of this research. I am a PhD student at Victoria University of Wellington in New Zealand. I am conducting research entitled: "The use of Information and Communication Technology in learning and teaching within Higher Education sector of a Small Island Developing State: The Case of the Maldives.

The main objectives of this research are: a) to explore the existing situation of ICT usage in higher education sector of Maldives; b) to discover how ICT can be better utilised in learning and teaching of Maldives higher education; and c) to devise a suitable model for future use in the higher education sector of the Maldives.

Participants are members of the higher education sector, IT sector and relevant agencies in the Maldives. I hope to get knowledge of the perceptions and expectations how ICT can be utilised in the higher education learning and teaching. The institution in which you work / study has given permission for you to participate in my research if you are willing.

Confidentiality of participating organisations and of participating individuals will be preserved as a matter of course. However, given the smallness of the higher 
education community in the Maldives, it may always be possible for members of the community to guess at the individuals and their organisations by the data they provide. This risk needs to be understood by participants but it will be minimised wherever possible.

The Human Ethics Committee of the School of Information Management at Victoria University of Wellington has granted ethical approval subject for this research. Participation is entirely voluntary; you may withdraw without having to give reasons at any stage of the research up until 01 July 2009. If you choose to withdraw, any material provided up to that point will be omitted from my research and destroyed or returned to you.

Your participation will be in the form of face to face digitally recorded interviews with me, and will last approximately 45-60 minutes. You will be able to check and if necessary amend transcripts of your interviews.

All raw data will be kept confidential to me and my supervisors. Information derived from the collected, collated and analysed data may be published as case studies, articles in academic journals, and presented at conferences. The thesis will be submitted for marking to the School of Information Management and deposited in the university library. In addition, a copy of the thesis will be lodged in Victoria University of Wellington electronic repository.

Any information or opinion that you may provide will not be attributed to you personally, and neither you nor your institution will be identified in any way. Throughout the project, raw data will be kept under password protection and destroyed two years after the completion of the project.

The attached consent form that includes a request for permission to audio-record interviews is attached. If you agree to participate, please complete the form, sign it, and return it to me. 
If you have any questions or would like to receive further information about the project, please contact Mohamed Kinaanath on +960 7775353 or via email at mohamed.kinaanath@vuw.ac.nz or my supervisors

1. Associate Professor, Dr. Hans Lehmann, at the School of Information Management at Victoria University of Wellington, by phone: + 6444635879 or via email hans.lehmann@vuw.ac.nz or via post.

2. Senior Lecturer, Dr. Brian Harmer, at the School of Information Management at Victoria University of Wellington, by phone: + 6444635887 or via email brian.harmer@vuw.ac.nz or via post.

My Contact Details: Mr. Mohamed Kinaanath

$\mathrm{PhD}$ Candidate

M. Gaahaka

Shaheed Ali Hingun

Male', Maldives

Phone: +960 7775353

e-mail: mohamed.kinaanath@vuw.ac.nz

OR mkinaanath@gmail.com

Best Regards

Mohamed Kinaanath 


\title{
Appendix 9: Consent for Interviews
}

\author{
Victoria \\ UNIVERSITY OF WELLINGTON \\ Te Whare Wānanga \\ o te Ūpoko o te Ika a Māui \\ 590 \\ Victoria University of Wellington \\ School of Information Management \\ Consent to participate in Research - Interviews
}

Project Title: THE USE OF INFORMATION AND COMMUNICATION TECHNOLOGY IN LEARNING AND TEACHING WITHIN HIGHER EDUCATION SECTOR OF A SMALL ISLAND DEVELOPING STATE: THE CASE OF THE MALDIVES

I have been given, and have understood an explanation of this research project. I have had an opportunity to ask questions and have had them answered to my satisfaction. I understand that I may withdraw myself (or any information I have provided) from the project without having to give reasons as long as I do it by 1 July 2009. I can do this by e-mailing Mohamed Kinaanath or his supervisor at the email address given below. I understand that if I withdraw from the project, any data I have provided will be returned to me or destroyed by Mohamed Kinaanath.

I understand that any information I provide will be kept confidential to the researcher to his supervisor. I understand that the information I have provided will be used only for this research project and that any further use will require my written consent. I understand that recorded interviews will be electronically wiped two years after the conclusion of the project.

Confidentiality of participating organisations and of participating individuals will be preserved as a matter of course. However, given the smallness of the higher education community in the Maldives, it may always be possible for members of the community to guess at the individuals and their organisations by the data they provide. This risk needs to be understood by participants but it will be minimised wherever possible. 
[Please mark with a $(\checkmark)$ to indicate agreement]

- I agree to take part in this research

- I understand that interviews will be audio recorded

- I will have an opportunity to check the transcripts of the interviews

- I would like to receive a summary of the results of this research when it is completed

- I understand that the data I provide will not be used for any other purpose or released to others without my written consent

- I understand a copy of the thesis will be lodged in Victoria University of Wellington electronic repository

Signature:

Name of the participant:

Date: 


\title{
Appendix 10: Information sheet for Focus Group Discussion
}

\author{
Victoria \\ UNIVERSITY OF WELLINGTON \\ Te Whare Wānanga \\ o te Ūpoko o te Ika a Māui

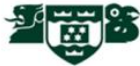 \\ Victoria University of Wellington \\ School of Information Management \\ Information Sheet - Focus Groups
}

Dear [participant]

[Date]

Project Title: THE USE OF INFORMATION AND COMMUNICATION TECHNOLOGY IN LEARNING AND TEACHING WITHIN HIGHER EDUCATION SECTOR OF A SMALL ISLAND DEVELOPING STATE: THE CASE OF THE MALDIVES

With the permission of your institution, I invite you to participate in this research. Let me first introduce myself and give you a brief overview of this research. I am a PhD student at Victoria University of Wellington in New Zealand. I am conducting research entitled "The use of Information and Communication Technology in learning and teaching within Higher Education sector of a Small Island Developing State: The Case of the Maldives, and I seek your participation as a member of a focus group.

The main objectives of this research are: a) to explore the existing situation of ICT usage in higher education sector of Maldives; b) to discover how ICT can be better utilised in learning and teaching of Maldives higher education; and c) to devise a suitable model for future use in the higher education sector of the Maldives.

Participants are members of the higher education sector, IT sector and relevant agencies in the Maldives. I hope to get knowledge of the perceptions and expectations how ICT can be utilised in the higher education learning and teaching. The institution in which you work / study has given permission for you to participate in my research if you are willing.

Confidentiality of participating organisations and of participating individuals will be preserved as a matter of course. However, given the smallness of the higher 
education community in the Maldives, it may always be possible for members of the community to guess at the individuals and their organisations by the data they provide. This risk needs to be understood by participants but it will be minimised wherever possible.

The Human Ethics Committee of the School of Information Management at Victoria University of Wellington has granted ethical approval subject for this research. Participation is entirely voluntary; you may withdraw without having to give reasons at any stage of the research up until 01 July 2009. If you choose to withdraw, any material provided up to that point will be omitted from my research and destroyed or returned to you.

The institution in which you work / study has given permission for you to participate in my research, however, participation is voluntary. If you decide to withdraw after focus group has commenced on 27th May 2009, it will not be possible to exclude any material you have already provided - this is because of difficulties of removing one participant's comment from group transcripts. In addition, participants are asked not to disclose opinions / information offered during the focus groups to any other person or party.

Focus groups participants will be either teachers or students. I hope to get knowledge of the perceptions and expectations how ICT can be utilised in the higher education learning and teaching. The focus groups take approximately 180 minutes with a coffee break. I also have got permission from your executive to review relevant documents of your organisation in order to gather more information on the subject. Wherever these are confidential I will respect that.

All raw data will be kept confidential to me and my supervisors. Information derived from the collected, collated and analysed data may be published as case studies, articles in academic journals, and presented at conferences. The thesis will be submitted for marking to the School of Information Management and deposited in the university library. In addition, a copy of the thesis will be lodged in Victoria University of Wellington electronic repository. 
Your focus groups sessions will be both audio-recorded and video-recorded with your permission. The attached consent form that includes a request for permission to audio-record and video-record the focus groups sessions is attached. If you agree to participate, please complete the form, sign it, and return it to me personally.

If you have any questions or would like to receive further information about the project, please contact Mohamed Kinaanath on +9607775353 or via email at mohamed.kinaanath@vuw.ac.nz or my supervisors

1. Associate Professor, Dr. Hans Lehmann, at the School of Information Management at Victoria University of Wellington, by phone: + 6444635879 or via email hans.lehmann@vuw.ac.nz or via post.

2. Senior Lecturer, Dr. Brian Harmer, at the School of Information Management at Victoria University of Wellington, by phone: + 6444635887 or via email brian.harmer@vuw.ac.nz or via post.

My Contact Details: Mr. Mohamed Kinaanath PhD Candidate

M. Gaahaka

Shaheed Ali Hingun

Male', Maldives

Phone: (960) 7775353

e-mail: mohamed.kinaanath@vuw.ac.nz

OR mkinaanath@gmail.com

Best Regards

Mohamed Kinaanath 


\title{
Appendix 11: Consent Focus Group Discussions
}

\author{
Victoria \\ UNIVERSITY OF WELLINGTON \\ Te Whare Wānanga \\ o te Ūpoko o te Ika a Māui

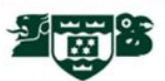 \\ Victoria University of Wellington \\ School of Information Management \\ Consent to participate in Research - Focus Groups
}

Project Title: THE USE OF INFORMATION AND COMMUNICATION TECHNOLOGY IN LEARNING AND TEACHING WITHIN HIGHER EDUCATION SECTOR OF A SMALL ISLAND DEVELOPING STATE: THE CASE OF THE MALDIVES

I have been given, and have understood an explanation of this research project. I have had an opportunity to ask questions and have had them answered to my satisfaction. I understand that I may withdraw myself (or any information I have provided) from the project without having to give reasons as long as I do it by 1 July 2009. I also understand that even if I choose to withdraw after the commencement of the focus groups 27 May 2009, it will not be possible to separate information I have provided from the records of the group. I can withdraw by emailing Mohamed Kinaanath or his supervisor at the email address given below. I understand that if I withdraw from the project, any data I have provided will be returned to me or destroyed by Mohamed Kinaanath except as noted above.

I understand that any information I provide will be kept confidential to the researcher to his supervisor. I understand that the information I have provided will be used only for this research project and that any further use will require my written consent. I understand that the recording of focus groups will be electronically wiped two years after the conclusion of the project.

Confidentiality of participating organisations and of participating individuals will be preserved as a matter of course. However, given the smallness of the higher education community in the Maldives, it may always be possible for members of the community to guess at the individuals and their organisations by the data they provide. This risk needs to be understood by participants but it will be minimised wherever possible. 
[Please mark with a $(\checkmark)$ to indicate agreement]

- I agree to take part in this research

- I understand that the focus group session(s) will be audio- and video recorded.

- I would like to receive a summary of the results of this research when it is completed

- I understand that the data I provide will not be used for any other purpose or released to others without my written consent

- I understand a copy of the thesis will be lodged in Victoria University of Wellington electronic repository

- I agree to keep confidential all opinions / information offered during the focus groups and not to disclose them to any other person.

Signature:

Name of the participant:

Date: 


\section{Appendix 12: Sample Interview Transcript}

Label:

Principal Researcher:

Project Title:
Information Technology Executive

Mohamed Kinaanath

The use of Information and Communication Technology in learning and teaching within Higher Education sector of a Small Island Developing State: The Case of the Maldives

\section{EXISTING ICT USAGE}

1. To your knowledge what is the existing ICT usage within the higher education sector or generally among people in the Maldives?

If we make a start we have starting utilising ICT in accordance with the Information Technology Master Plan. When we formulated the Information Technology Master Plan we noticed that we were in a changing phase and basically information technology is utilised for a very low-end work.

This is evident even from the higher education sector, government sector; and private sector usually computers treat as an extension of a typewriter. We are now moving from this kind of ICT existing phase to a new ICT phase and we are working to change the existing culture to a new culture of ICT usage.

\section{NATIONAL CONTEXT (THE MALDIVES)}

1. In the Maldives ICT or IT is used in several sectors (i.e. education, health etc). What are the country level barriers we are facing in the use of IT?

Firstly the biggest barrier in utilising information technology in the best way; and most effective way is the lack of competent human resources.

We do not have enough competent human resources who can lead, drive and in required numbers within all the organisations and also at MCHE. There is a lack of competent human resources to support the critical mass in the Maldives. 
Every organisations has got little human resources and do not have enough human resources with the required critical mass and thus therefore there is huge difficulty in implementing information technology at a considerable speed.

I am not talking about the difficulties faced in terms of ICT hardware wise. In terms of ICT hardware wise I would consider the Maldives would be considered as a leading country.

I am talking about the change in human element, self side and change in mindset aspects. We are facing difficulties in the change in mindset. There is a huge problem faced in not having critical mass to drive of human resources in driving information technology in the required fashion.

\section{What steps are already taken in developing human resource especially in the e-Government Project of the Maldives?}

From human resources side I would consider this as a huge challenge. We knew we need to development human resources even when we initially started this e-Government project.

Human development is a critical area and we do not face human development issue not only from the information technology sector.

The human development for the information technology sector is mainly focused in academic arenas. The degree graduates are not there, masters graduates are not there and diploma graduates are also not there but instead people try to go for further studies and basically nobody is retained. 


\section{Given the resources why do you think ICT is underutilised in the Maldives? Please describe.}

We need to increase the ICT capacity of the people and we should demonstrate the anticipated benefits of ICT at a critical level and therefore ICT utilisation becomes quite difficult.

Based on the increase access penetration our goal should be targeted we should show and demonstrate the anticipated ICT benefits that we get when ICT is used.

In addition, ICT is underutilised because the development work in utilising ICT is extremely high. For example: there is no driving personnel's at MCHE the delivering online learning; develop materials; developing learning environment; and develop online systems at Centre for Opening Learning (COL); and developing electronic system.

The existing staffs have other commitments and staffs do not have time to concentrate on developing ICT enabled systems. There is a lack of people and most people believe in technological wise it is not a difficult task to implement. There are lot of open source resources available such as software's for us to setup systems easily.

As you have mentioned we are not too bad in terms of ICT hardware infrastructures. We have the ICT hardware and we have necessary ICT facilities with least cost but even though if we have the ICT facilities still cannot start utilise the ICT resources.

We have the hard task of buy-in, selling process of these ICT facilities and create ICT awareness to people. When a new technology is introduced we need lot of time and if we need to speed up the process we definitely lot of campaigning and huge workload. 
For example: the rate of embracing mobile phone technology is high compared to other technologies. Mobile phone by nature is a simple device with easy to use functions like the mobile phones by pressing few buttons. Although we have a very high mobile phone penetration but we use the very basic features of mobile phones. In terms of mobile phone capacities and use of extra more functions, I would consider we are very far behind. But if we look at the mobile penetration rate we will be considered as one of the highest countries in the world. Someone has to take an initiative to explain the high-end functionalities which are in-built in the mobile technologies.

We need to explore innovative ways to increase the capacity of the people in order to use these new technologies. In the case of mobile phones people take their own initiatives and self driving because other people are use it and they can see the benefit.

Benefit should drive the ICT usage and if we can drive from these benefits the penetration rate should increase.

\section{As you have said the mobile penetration rate is high and it is close to half million users. Can't we use the mobile service for learning tool if we can learn the complicated functions of the mobile devices with no cost?}

Mobile technologies by nature is used in many countries and used for different purposes. With the help of internet and mobile technologies are already merged which can perform different tasks.

With the use of mobile technologies we do have some limitations such as: availability of space and mobile usage. Given the limitations we can utilise appropriately by using mobile devices in the learning environment. 


\section{Appendix 13: Sample Focus Group Transcript}

\section{National Context (The Maldives)}

1. What are the perceived barriers currently faced in dealing and using ICT in the higher education learning and teaching in the unique geographical context of the Maldives? Please explain

Student - Focus Group: There are about 200 inhabited islands in the Maldives. To my knowledge there are islands with a population of about 300 people. Even if the populations are low we need to provide ICT services to these low density populated islands and provide Internet services to all these islands.

Student - Focus Group: I think there is huge "cost" involved and this is one major problem. The Maldives has small economy and there are growing problems in the economy due to budgetary constraints. Despite having a small economy, the Maldives should provide all basic services to the remote islands but the cost factor is a major problem encountered by the Government of the Maldives.

Student - Focus Group: There are few people who have the necessary skills in utilising ICT. These few people cannot be catered to all these islands and we need skilled people urgently.

Moderator: What is the relationship between having required skills and dispersed island nation concept?

Student - Focus Group: The small sets of skilled people have to go to all these islands for training with a huge cost involved and we need time as well.

Student - Focus Group: The transportation between the islands is mostly by sea. The cost of travelling by air is very high and simply unaffordable to our 
citizens. The basic ICT infrastructures does not exist and I don't think there is an initiation or thought of setting up ICT infrastructures.

Student - Focus Group: The major barrier is that we do not have good transportation system in the Maldives. The islanders have to come to Male' for higher education and there are few people who can afford to stay in Male' due to increased costs in accommodation. Due to financial difficulties there are lot of students in the islands who cannot study at tertiary level but wanted to study.

Student - Focus Group: The institutes and companies are not making the services available to the whole country due to low population densities in the islands and thus companies resist investing.

Moderator: What are you referring to low population?

Student - Focus Group: The island populations are low. For example: developing an online learning centre in these low density populated islands is not worth due to huge investment.

Student - Focus Group: The other point is islanders who are going to Male' for higher education studies are not returning back to their islands after their studies and it is because there are no opportunities in the islands for career development. 


\section{Appendix 14: NViVo tree nodes sample}

\section{Tree Nodes}

Name

它.

Geography

Geographically and remoteness as a challenge

Geography as a challenge for communication

Geography hinders development

Geography incurs huge cost

ICT access limited due to geography

Island nation with islands geographically dispersed

Maldives geography is different from other countries

Sea Level rise and global warming vulnerability

G. ICT as the final solution

ICT as a life saver

ICT importance in the Maldives higher education

ICT should be a must in the Maldives higher education

ICT will reduce the geographical barrier with double outcome

$\square . \bigcirc$ Islands

Island families migrating to Male' for opportunities

No ICT awareness in the islands

No services and opportunities at island due to smallness

Not all students have tertiary education opportunities

Remote islands are very small

Travelling from islands to islands was difficult

ظ. Male'

Everyone coming to Male' due to high developmental work

Heavy reliance on Male'

High costs in Male' and not everyone islander can afford

Male' is most densely population cities in the world

Overcrowding in Male 


\section{Appendix 15: Conceptualisation Sample Diagrams}

\section{Sample Diagram 1}

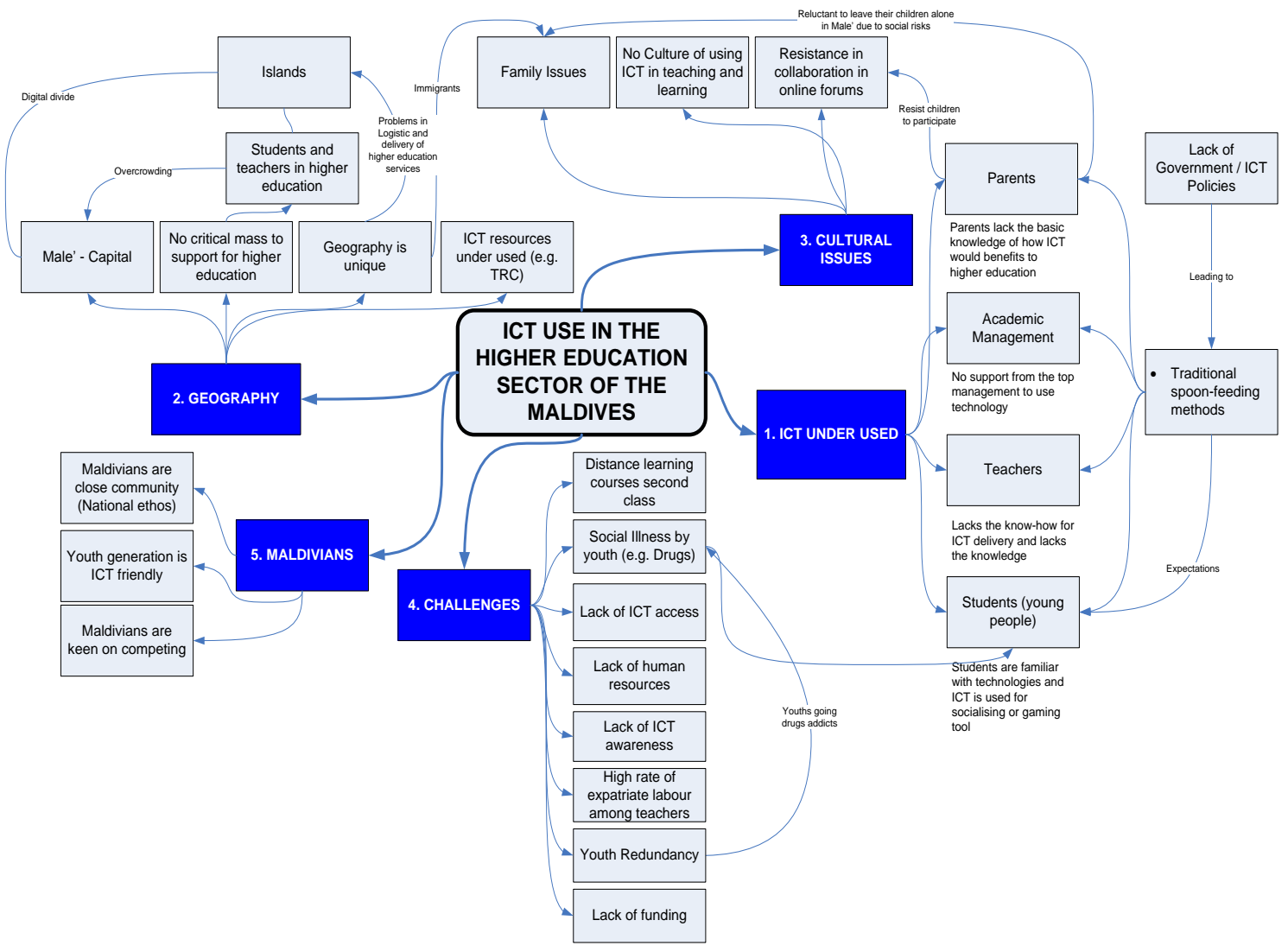




\section{Sample Diagram 2}

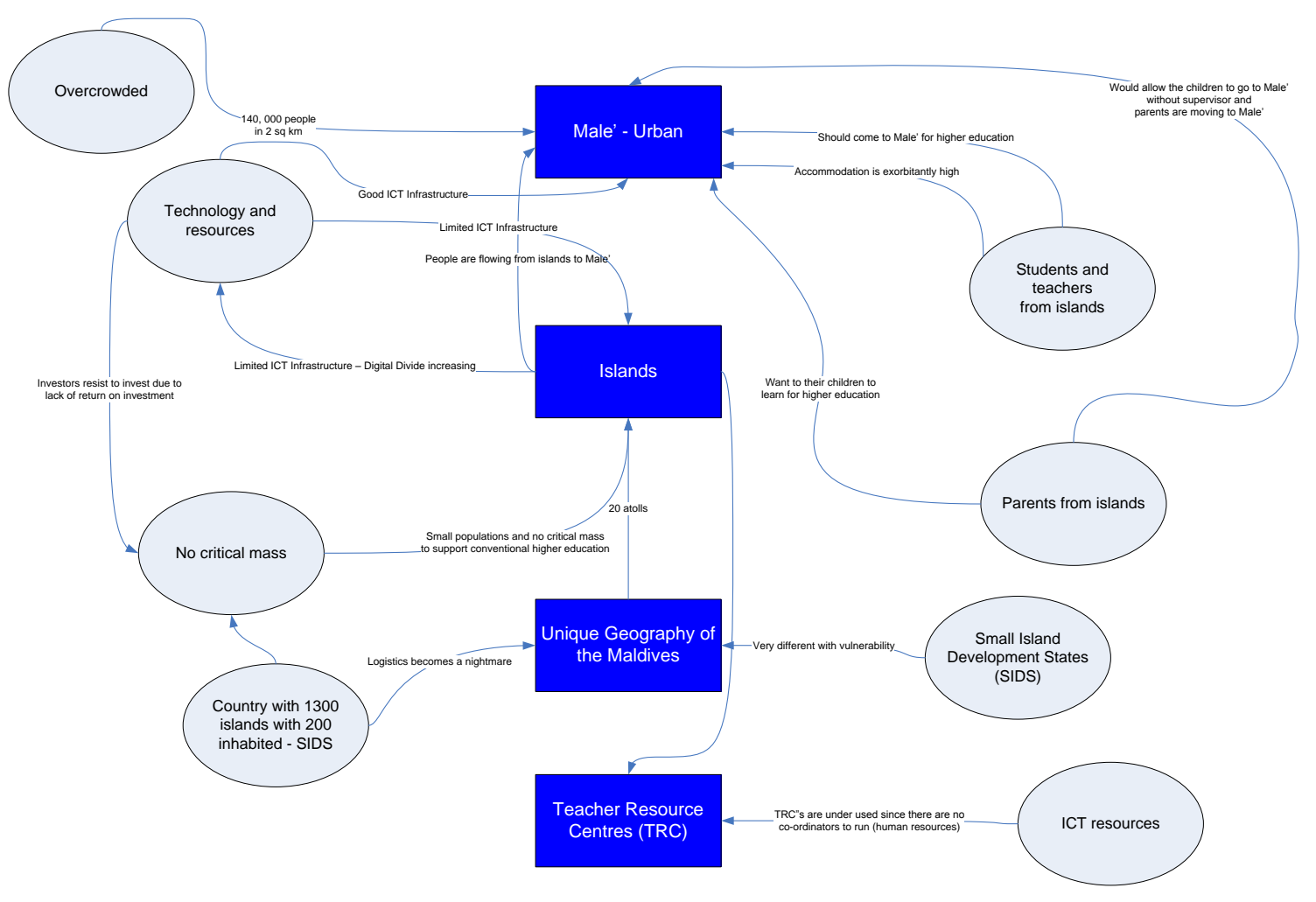

